\title{
Involutive Moving Frames
}

\author{
A THESIS \\ SUBMITTED TO THE FACULTY OF THE GRADUATE SCHOOL \\ OF THE UNIVERSITY OF MINNESOTA \\ BY
}

Orn Arnaldsson

IN PARTIAL FULFILLMENT OF THE REQUIREMENTS

FOR THE DEGREE OF

DOCTOR OF PHILOSOPHY

PETER J. OLVER

June, 2017 
(C) Orn Arnaldsson 2017

ALL RIGHTS RESERVED 


\section{Acknowledgements}

A lot of people deserve a lot of credit for this thesis. Ásta, for everything. Björk, for everything else. Alex - it never would have worked without him. Anna and Arnaldur, my parents, for their encouragement and support. Baggi, for being the best uncle, and Siggi and Áslaug, who I am very fortunate to have as in-laws. Don, for the good company at Solstice. Erin Oakley, for the green BMW. My fellow graduate students, the staff and faculty at the University of Minnesota, for making $\mathrm{U}$ of $\mathrm{M}$ such a great place.

I was lucky enough to receive support for graduate school from two fellowship funds. Hrafnkelssjóður and the Leifur Eiríksson foundation. I express my sincere gratitude to these wonderful organizations.

I wish to take the opportunity to thank Bernard Deconinck, for introducing me to symmetry methods and his kind help in getting me into the $\mathrm{U}$ of $\mathrm{M}$.

Finally, I would like to thank my adviser, Peter Olver, for his books, papers, and guidance, all of which will remain a source of inspiration for what will follow. 


\section{Dedication}

Til Ástu og Bjarkar. Takk fyrir allt. 


\begin{abstract}
This thesis combines two major equivalence methods, Cartan's equivalence method and the method of the equivariant moving frame for pseudo-groups, to obtain a more efficient tool with which to tackle a large class of equivalence and symmetry problems. These include, for example, all equivalence problems for sections of tensor bundles under change of variable and those arising in the calculus of variations. Furthermore, once the connection between the two original equivalence methods has become clear, we provide a proof of termination of Cartan's method in these cases. To obtain this termination result, we develop a novel algorithm for the computation of Pommaret bases for polynomial modules.
\end{abstract}




\section{Contents}

\begin{tabular}{ll}
\hline Acknowledgements & i
\end{tabular}

Dedication ii

Abstract iii

\begin{tabular}{lll}
\hline & Introduction & 1
\end{tabular}

1.1 Cartan's equivalence method . . . . . . . . . . . . . . . . . 1

1.2 Pommaret bases . . . . . . . . . . . . . . . . . . . 3

$1.3 \quad$ Equivariant moving frames for pseudo-groups . . . . . . . . . . 4

1.4 The combined equivalence method. . . . . . . . . . . . . 5

1.5 Structure of the thesis . . . . . . . . . . . . . . . . 6

\begin{tabular}{lll|}
2 & A Pommaret basis algorithm & 8
\end{tabular}

2.1 Involutive divisions . . . . . . . . . . . . . . . . . . . . . . . . . 8

2.2 Involution algorithms . . . . . . . . . . . . . . . . . . . . . 12

2.3 Coordinate independent quantities . . . . . . . . . . . . . . 17

2.4 Reduced Cartan characters . . . . . . . . . . . . . . . . . . 24

2.5 Examples . . . . . . . . . . . . . . . . . . . . . . 27

3 Cartan-Kuranishi completion 32

$3.1 \quad$ Completion for formally integrable equations . . . . . . . . . . . . 37

$3.2 \quad$ A general completion algorithm . . . . . . . . . . . . . . . . 40 
3.3 Cartan normal form and regularity . . . . . . . . . . . . . . . . . 48

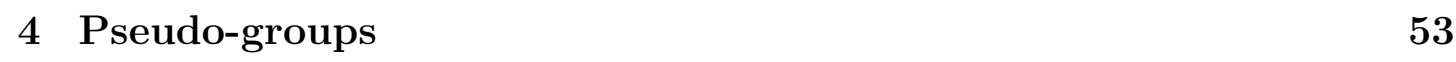

4.1 Basic objects . . . . . . . . . . . . . . . . . . . . . . 53

4.2 Lifted invariants . . . . . . . . . . . . . . . . . . . 60

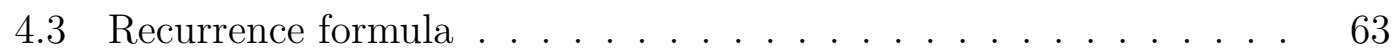

$\begin{array}{|ll|}5 & \text { Equivalence of sections }\end{array}$

$5.1 \quad$ A group modification of Algorithm 3.2 .6 . . . . . . . . . . . . . 68

5.2 The equivariant moving frame . . . . . . . . . . . . . . . . . . 73

5.3 Sections of $\mathcal{G}_{p} \ldots \ldots \ldots$. . . . . . . . . . . . . . . . . . 78

$5.4 \quad G$-structures and partial moving frames . . . . . . . . . . . . . . . 87

5.5 Structure of a partial moving frame . . . . . . . . . . . . . . . . . 100

5.6 Exterior differential systems . . . . . . . . . . . . . . . . . . . . . 110

\begin{tabular}{llr}
\hline & Examples & 119
\end{tabular}

6.1 Equivalence of second order ordinary differential equations . . . . 120

6.2 Divergence equivalence of Lagrangians . . . . . . . . . . . . . . . 126

6.3 Medolaghi's pseudo-group . . . . . . . . . . . . . . . . . . . 130

6.4 Equivalence of differential operators . . . . . . . . . . . . . . . . . 134

$\begin{array}{lll}7 & \text { More general group actions } & 138\end{array}$

7.1 Congruence in a Lie group, a groupoid approach . . . . . . . . . . 139

\begin{tabular}{lr}
\hline Bibliography & 147
\end{tabular}

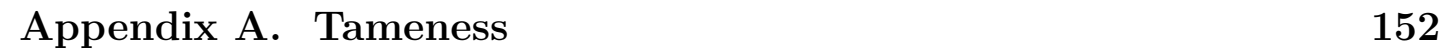

A.1 Linearization . . . . . . . . . . . . . . . . . . 154

A.2 Tameness of Lie pseudo-groups . . . . . . . . . . . . . . 158 


\section{Chapter 1}

\section{Introduction}

The central goal of this thesis is developing an equivalence method that combines Cartan's equivalence method and the method of the equivariant moving frame for pseudo-groups. We reach this goal for equivalence problems arising from what we call horizontal Lie pseudo-group actions. These actions already cover many equivalence problems of interest such as those arising in the calculus of variations and equivalence between sections of tensor-bundles (e.g. Riemannian metrics). The extension of our results to more general equivalence problems will appear in a forthcoming research paper. Along the way we develop some completion algorithms for differential equations by way of a novel algorithm for the computation of Pommaret bases. We divide this introduction into subsections to discuss the different ingredients in this modified equivalence method, and its practical value in solving equivalence problems.

\subsection{Cartan's equivalence method}

The collection of local diffeomorphisms that preserve some geometric structure on a manifold usually forms an infinite dimensional pseudo-group rather than a finite dimensional Lie group. Characterizing these transformations is the equivalence problem for the geometric structure at hand. In studying these infinite 
dimensional analogs of Lie groups, Élie Cartan solved, [7], the general equivalence problem for coframes. This equivalence problem included all known equivalence problems as special cases, but this (perhaps) surprising situation is explained by the fact that all equivalence problems take place in the diffeomorphism groupoid whose Maurer-Cartan forms, properly restricted to the equivalence problem at hand, completely characterize the equivalence maps (see Section 5.4). Cartan's solution also necessitated the concurrent development of the theory of exterior differential systems, culminating in the celebrated Cartan-Kähler theorem which extends the Cauchy-Kovalevskaya theorem on the local solvability of analytic systems of differential equations that can be written in normal form to those that can be completed to their involutive form, [46]. Every system of differential equations can be completed to its involutive form (perhaps after a change of independent variables). This is the Cartan-Kuranishi theorem. However, although Cartan's equivalence method has, on the face of it, many things in common with the completion process of a differential equation, it has never been actually proven that it terminates with an involutive exterior differential system, although this was conjectured by Cartan. The source of difficulty in proving termination is the fact that the increasing system of differential forms constructed during Cartan's method does not directly possess the symbol structure of a differential equation. In this thesis, restricting to so called horizontal Lie pseudo-group actions, we relate the structure of the differential forms of Cartan to the structure of a certain differential equation, and prove that Cartan's method terminates at involution at the same time as this differential system, (see Section 5.6). Our result is also more general; we do not require restricting to equivalence problems of constant type which has been a tendency in some previous treatments, e.g. [14].

Cartan's equivalence method was applied by his disciples, most notably S.S. Chern in proving the generalized Gauss-Bonnet theorem, [9], but, mostly because of its immense computational complexity, was not a very active area of research until the 1980s when its importance in solving a variety of equivalence problems in differential equations $([4,22,26])$, calculus of variations $([3,15,19,25])$ and control 
theory ([13]) became clear. However, the complexity of the calculations involved in solving an equivalence problem using the method has remained its greatest drawback, even with the aid of modern computational algebra systems. We shall see how the modern theory of equivariant moving frames for pseudo-groups helps in decreasing the computational load of the original method.

\subsection{Pommaret bases}

Involution of a system of differential equations is a condition on the symbol module of the system that guarantees local solvability, and, in principle, allows for the determination of a local power series solution. Originally, [33], this condition was described by the vanishing of certain Koszul homology groups of the symbol module (or, dually, its Spencer cohomology groups). The algebra involved with the computations of these (co)homology groups is very heavy indeed as evidenced by the fact that the Spencer-Kodaira approach, ([28, 29, 30]), to the geometry of differential equations, and the following monograph, [32], on Lie pseudo-groups essentially ended research into these matters. Recently, building on the classic, early twentieth century work of Janet and Riquier ([21, 45]), a more constructive approach to involution has been developed based on involutive divisions, [17. These lead to special types of Gröbner bases (the involutive bases) with extra, combinatorial, properties, that allow for a practical completion process that we take advantage of. The involutive division that most naturally lends itself to analysis of differential equations is the Pommaret division. A central problem in the theory of Pommaret bases is existence of such bases: a Pommaret basis does not exist in all variables, so a smart change of variables may be needed before being able to compute such a basis. In Section 2 we develop a novel algorithm that computes $\delta$-regular coordinates, i.e. coordinates in which a Pommaret basis exists. This algorithm is tailor-made to apply to Cartan's equivalence method in later sections and as such it requires computing maximal ranks of symbolic matrices. 
We should mention that Pommaret bases have found a stunning number of applications in computational algebraic geometry. This is due to the fact that many geometric invariants can be directly read off such a basis, including dimension, depth and Castaelnuovo-Mumford regularity, [46]. It remains to be seen whether our algorithm has applications in the realm of algebraic geometry.

\subsection{Equivariant moving frames for pseudo-groups}

Inspired by another invention of Élie Cartan, the method of moving frames, 8], Olver and Fels developed a theory that provided theoretical underpinnings for Cartan's method while extending it considerably; The equivariant moving frame for a Lie group, $G$, acting on a manifold, [12]. This is a powerful tool for studying geometric properties of submanifolds and their invariants under the Lie group action and has found a plethora of applications. In particular, the equivariant moving frame computes a $G$-coframe for each submanifold in its domain which completely determines the congruence problem of submanifolds. Other applications include classical invariant theory $([1,37])$, object recognition and symmetry detection $([2,5])$, invariant finite difference numerical schemes ([6, 39]) and computing minimal generating sets of differential invariants ([40, 20]). In [31] the equivariant moving frame was used to solve the problem of constructing the invariant Euler-Lagrange equations from an invariant Lagrangian. Applications to the evolution of differential invariants under invariant submanifold flows, leading to integrable soliton equations and signature evolution in computer vision, can be found in [27, 41].

In the series of papers [42, 43, 44], Olver and Pohjanpelto extended the equivariant moving frame to the infinite dimensional realm of a Lie pseudo-group, $\mathcal{G}$, of local diffeomorphisms of a manifold, $\mathcal{X}$, and provided a practical structure theory of these infinite dimensional analogs of Lie groups. Lie pseudo-groups are determined by systems of differential equations and their approach relied on the fact that the subset of the jet bundle that these equations determine carries a 
groupoid structure. The equivariant moving frame for pseudo-groups generalizes its Lie group counterpart since Lie groups are special cases of Lie pseudo-groups, but to solve the congruence problem of submanifolds in $\mathcal{X}$ under the action of $\mathcal{G}$ the authors needed a result they called persistence of freeness of Lie pseudogroups, [44]. In fact, the equivariant moving frame for pseudo-groups has had to rely on the pseudo-group action eventually becoming free after prolonging to a jet space of high enough order (persistence of freeness then indicates that the action on all jet spaces above this order is also free) in order to say something meaningful about the equivalence problem at hand. In particular, the equivariant moving frame has until now not utilized Cartan's theory of involution. One purpose of this thesis is to take the first steps towards embedding the theory of involution into the equivarant moving frame. To this end, we prove the Lie pseudo-group analog of a result on congruence of submanifolds in a Lie group that underlies Cartan's equivalence method and the equivariant moving frame approach, see Theorem 5.3.4. We then take advantage of the flexibility of exterior differential systems (compared to differential equations) to obtain a proof of termination of Cartan's equivalence method for a large class of equivalence problems.

\subsection{The combined equivalence method}

It has remained somewhat mysterious how Cartan's equivalence method and the equivariant moving frame for pseudo-groups are related, even though both methods compute the same invariants, coframes and invariant derivatives. Cartan's method is built on computing the exterior derivative of coframes and analyzing the structure coefficients that appear while the equivariant moving frame has relied on the explicit computation of lifted invariants. Both methods have their pros and cons. The chief difficulty in implementing the equivariant approach is that the formulas for lifted invariants can rapidly get out of hand, overwhelming even computer algebra systems, while Cartan's method requires the solution of a rather large linear system of equations at each step of the method. One contribution of 
this thesis is to clarify the precise relationship between the two methods and combining them into an equivalence method that uses the best from both worlds. In particular, the so-called intrinsic method, popularized by Gardner, [14, can be employed without the risks it once carried of leading one down "spurious branches of the equivalence problem" (see [36, p. 359]). See Section 6 for examples where difficult equivalence problems are solved with relative ease using this combined method. In the final section we take the first step toward a completely general equivalence method, not requiring the pseudo-group to be horizontal, but development of this method is still underway. As an application, and a showcase, of the groupoid approach to Lie pseudo-groups we give a solution to the congruence problem of submanifolds under a finite dimensional Lie group action.

\subsection{Structure of the thesis}

We begin, in Section 2, by developing a novel algorithm for the computation of $\delta$-regular coordinates for polynomial modules. The key idea for this algorithm is computing an analog of Cartan's reduced characters for the modules. Understandably, this section has a very different flavor than the rest of the thesis. This algorithm has a direct application to the completion of differential equations to involution which is presented in Section 3 . Every version of the Cartan-Kuranishi theorem, [33, 46], needs to make certain assumptions of the regularity of the system in question. We give our own definition of regularity in Definition 3.0.6 and show, in Section 3.3, that it is, in fact, necessary for the application of the Cartan-Kähler theorem. Further, we show that all formally integrable systems are regular in a dense open set and in the appendix we shall prove that for Lie pseudo-groups this open dense set is actually invariant. In proving termination of Cartan's equivalence method, we shall need an "exterior calculus" version of our completion algorithm which we present in Remark 3.2.8.

Section 4 gives a rapid overview of the groupoid approach to Lie pseudo-groups as well as setting the stage for Cartan's equivalence method in the special case 
of horizontal actions. Section 5 forms the core of the thesis. It first introduces a group modification of the Cartan-Kuranishi completion algorithms from Section 3 and gives a quick review of the equivariant moving frame. Section 5.3 contains the fundamental proof of equivalence of sections in the groupoids associated with a Lie pseudo-group. The proof is similar to its Lie group counterpart, see e.g. [18], but this type of result has been missing in the equivariant moving frame calculus for Lie pseudo-groups. The following section shows how this theorem provides the classical $G$-structures of Cartan in the horizontal case, and connects Cartan's equivalence method and (partial) moving frames. Section 5.5 combines the group modified completion algorithms with partial moving frames and the recurrence formula to prove that Cartan's equivalence method terminates. We then end the chapter by showing how to construct equivalence maps, once Cartan's method has terminated, using the Cartan-Kähler theorem for exterior differential systems.

At this point in the thesis, having freed ourselves from the devil of the details, we apply our work to specific, and substantial (horizontal) examples. These include equivalence of second order ODE and divergence equivalence of first order Lagrangians under point transformations.

As mentioned above, the final section discusses the generalization of our methods to non-horizontal actions, but in order to prove termination of Cartan's method in general we need to grapple with the equivalence problem of submanifolds under a general pseudo-group action on the ambient space. Work on this general method is well underway and will be ready soon. As a warm-up for the general case, we give a groupoid oriented solution to the equivalence problem for finite dimensional Lie groups. This finite dimensional case actually turns out to have much in common with the general method, to be presented elsewhere. 


\section{Chapter 2}

\section{A Pommaret basis algorithm}

In this chapter we develop a new algorithm, based on the ideas in the original equivalence method of Cartan in differential geometry, for the calculation of a Pommaret basis for a polynomial ideal $\mathcal{I} \subset \mathcal{P}$, where $\mathcal{P}=\mathbb{k}\left[x^{1}, \ldots, x^{n}\right]$, and $\mathbb{k}$ is some infinite field. The correctness and termination of this algorithm will then directly apply to prove, in later sections, that Cartan's equivalence method terminates. The first subsection introduces the basic objects of study.

\subsection{Involutive divisions}

We assume throughout that Gröbner bases (including Pommaret bases), autoreductions and leading terms, monomials, and exponents are taken with respect to the degree reverse lexicographic order, where $x^{1}<x^{2}<\cdots<x^{n}$, and for a polynomial $f \in \mathcal{P}$ the last three are denoted by $\operatorname{lt}(f), \operatorname{lm}(f)$ and $\operatorname{le}(f)$, respectively. Let $\mathcal{P}^{(q)}$ denote the $\mathbb{k}$-linear space of $q^{\text {th }}$ degree homogeneous polynomials, $\mathcal{P}^{(\leq q)}$ denote the $\mathbb{k}$-linear space of all polynomials of degree $\leq q$ and $\mathcal{P}^{(\bar{q})}$ be the set of polynomials of degree exactly $q$. Also introduce the obvious projection operators $\pi_{q}: \mathcal{P} \rightarrow \mathcal{P}^{(q)}$ and $\pi_{\leq q}: \mathcal{P} \rightarrow \mathcal{P}^{(\leq q)}$. For a set $\mathcal{F} \subset \mathcal{P}$, we denote the $\mathbb{k}$-linear vector space it spans in $\mathcal{P}$ as $\langle\mathcal{F}\rangle_{\mathrm{k}}$. A linear change of variables $\bar{x}=A x$ gives an algebra isomorphism of $\mathcal{P}^{(q)}$ and $\mathcal{P}^{(\leq q)}$ with their counterparts in $\overline{\mathcal{P}}=\mathbb{k}\left[\bar{x}^{1}, \ldots, \bar{x}^{n}\right]$ 
and a bijection of $\mathcal{P}^{(\bar{q})}$ to its counterpart in the barred variables. For a multi-index $\mu \in \mathbb{N}_{0}^{n}$, we define $x^{\mu}$ in the obvious way, but we also sometimes identify $j$, for $1 \leq j \leq n$ with the multi-index with 1 in seat $j$, but zero elsewhere. This means, for example, that $\mu-j$ is the multi-index obtained by subtracting 1 from the $j^{\text {th }}$ coordinate of $\mu$. Lower-case Greek letters denote multi-indexes while lower case Latin letters are used to denote numbers in $\{1,2, \ldots, n\}$.

The set of multi-indexes, $\mathbb{N}_{0}^{n}$, coupled with compontwise addition form an abelian monoid, $\left(\mathbb{N}_{0}^{n},+\right)$. For $\nu \in \mathbb{N}_{0}^{n}$ we define its cone as the set $\mathcal{C}(\nu):=\nu+\mathbb{N}_{0}^{n}$. An ideal in $\mathbb{N}_{0}^{n}$ is a set $\mathcal{J} \subset \mathbb{N}_{0}^{n}$ such that $\mathcal{J}+\mathbb{N}_{0}^{n}=\mathcal{J}$. For a subset $\mathcal{B} \subset \mathbb{N}_{0}^{n}$, its span is the ideal

$$
\langle\mathcal{B}\rangle:=\bigcup_{\nu \in \mathcal{B}} \mathcal{C}(\nu)
$$

By Dixon's lemma [10], every ideal, $\mathcal{J}$, in $\mathbb{N}_{0}^{n}$ has a finite basis, i.e. a finite set $\mathcal{B}$ such that $\langle\mathcal{B}\rangle=\mathcal{J}$. An involutive division is a concept that aims to decompose a monoid ideal $\mathcal{J}$ into a finite union of disjoint cone-like sets. Its definition may, at first, seem convoluted but we provide some further motivation in the following remark.

Definition 2.1.1. An involutive division $L$ is defined on the abelian monoid $\left(\mathbb{N}_{0}^{n},+\right)$, if for any finite subset $\mathcal{B} \in \mathbb{N}_{0}^{n}$ and every $\nu \in \mathcal{B}$, a set $N_{L, \mathcal{B}}(\nu) \subset\{1, \ldots, n\}$ of multiplicative indices is defined and consequently a submonoid $M(\nu, \mathcal{B}):=$ $\left\{\mu \mid \forall j \notin N_{L, \mathcal{B}}(\nu): \mu_{j}=0\right\}$ such that the following hold for the involutive cones $\mathcal{C}_{L, \mathcal{B}}(\nu):=\nu+M(\nu, \mathcal{B})$.

1. For any two elements, $\nu, \mu \in \mathcal{B}$ such that $\mathcal{C}_{L, \mathcal{B}}(\nu) \cap \mathcal{C}_{L, \mathcal{B}}(\mu) \neq \varnothing$, then $\mathcal{C}_{L, \mathcal{B}}(\nu) \subset \mathcal{C}_{L, \mathcal{B}}(\mu)$ or $\mathcal{C}_{L, \mathcal{B}}(\mu) \subset \mathcal{C}_{L, \mathcal{B}}(\nu)$

2. If $\mathcal{B}^{\prime} \subset \mathcal{B}$, then $N_{L, \mathcal{B}} \subset N_{L, \mathcal{B}^{\prime}}$.

Remark 2.1.2. In words, the involutive division $\mathrm{L}$ is a rule that assigns to every pair $\mathcal{B} \subset \mathbb{N}_{0}^{n}$ and $\nu \in \mathcal{B}$ the set $N_{L, \mathcal{B}}(\nu)$. It is not obvious how this rather technical definition is related to our interest in decomposing monoid ideals into disjoint cones, but it is in fact tailor-made for such a decomposition. Here's how. 
For a finite basis, $\mathcal{B}$, for an ideal $\mathcal{J}$, and an involutive division $L$ on $\mathbb{N}_{0}^{n}$, we define the involutive span of $\mathcal{B}$ with respect to the division $L$ to be the union of the involutive cones of the elements of $\mathcal{B}$,

$$
\langle\mathcal{B}\rangle_{L}:=\bigcup_{\nu \in \mathcal{B}} \mathcal{C}_{L, \mathcal{B}}(\nu)
$$

If the involutive span of $\mathcal{B}$ happens to equal the whole ideal $\mathcal{J}$ (warning: for a given set $\mathcal{B}$, finding an involutive basis for $\langle\mathcal{B}\rangle$ is in general not an easy problem), then we say that $\mathcal{B}$ is a weak involutive basis for $\mathcal{J}$. Now, thanks to conditions 1. and 2. in Definition 2.1.1, we can actually reduce the (weak) basis $\mathcal{B}$ to a strong involutive basis $\mathcal{B}^{\prime}$ where all the involutive cones in $\left\langle\mathcal{B}^{\prime}\right\rangle_{L}$ are disjoint. To see this, assume that two cones in $(2.2), \mathcal{C}_{L, \mathcal{B}}(\nu)$ and $\mathcal{C}_{L, \mathcal{B}}\left(\nu^{\prime}\right)$, intersect. By condition 1. above, one is included in the other, say $\mathcal{C}_{L, \mathcal{B}}\left(\nu^{\prime}\right) \subset \mathcal{C}_{L, \mathcal{B}}(\nu)$. Now simply drop $\nu^{\prime}$ from the weak basis $\mathcal{B}$ to obtain the set $\mathcal{B}_{1}:=\mathcal{B} \backslash\{\nu\}$. By condition 2. above, in the involutive span $\left\langle\mathcal{B}_{1}\right\rangle_{L}$, the involutive cones have all increased and so $\langle\mathcal{B}\rangle_{L} \subset\left\langle\mathcal{B}^{\prime}\right\rangle_{L}$. But both are included in the ideal $\mathcal{J}$ so $\mathcal{B}^{\prime}$ is indeed a weak basis for $\mathcal{J}$. There are only finitely many elements in $\mathcal{B}$, so continuing this process will eventually lead to a set $\mathcal{B}^{\prime}$, whose involutive span is still $\mathcal{J}$, and all of whose involutive cones are disjoint.

The most important involutive division for our purposes is the Pommaret division, denoted by $P$, and defined as follows. For a multi-index $\nu$, define its set of multiplicative variables $N_{P}(\nu):=\{1,2, \ldots, \operatorname{cls}(\nu)\}$, where $\operatorname{cls}(\nu):=\min \left\{i \mid \nu_{i} \neq\right.$ $0\}$, is the class of the multi-index $\nu$. Proving that this actually gives a division is an easy exercise. Notice how $P$ does not depend on the set $\mathcal{B}$ which $\nu$ belongs to. Such divisions are called global. This property is convenient for computations: In the process of determining a Pommaret basis, we do not need to constantly calculate the change in multiplicative indices as we add or eliminate elements from our potential basis (cf. condition 2. in Definition 2.1.1.)

We mention here another division, the Janet division, denoted by $J$, whose surprising connection to the Pommaret division facilitates the existence theory of 
Pommaret bases (see [46, Chapter 4.3]). It is defined as follows. Let $\mathcal{B} \subset \mathbb{N}_{0}^{n}$, and $\nu \in \mathcal{B}$. For $\left(d_{k}, d_{k+1}, \ldots, d_{n}\right) \in \mathbb{N}_{0}^{n-k+1}$ define the set

$$
\left(d_{k}, d_{k+1}, \ldots, d_{n}\right)_{\mathcal{B}}:=\left\{\nu \in \mathcal{B} \mid \nu_{i}=d_{i}, k \leq i \leq n\right\} .
$$

We declare the index $l$ to be multiplicative for $\nu$ in $\mathcal{B}$ if $\nu_{l}=\max \left\{\mu_{l} \mid \mu \in \mathcal{B}, \mu \in\right.$ $\left.\left(\nu_{l+1}, \ldots, \nu_{n}\right)_{\mathcal{B}}\right\}$.

Example 2.1.3. Let $\mathcal{B}=\{a, b, c\}=\{(2,0,1),(1,1,0),(1,0,2)\} \subset \mathbb{N}_{0}^{3}$. Then

$$
N_{P}(a)=N_{P}(b)=N_{P}(c)=\{1\},
$$

while

$$
N_{J, \mathcal{B}}(a)=N_{J, \mathcal{B}}(b)=N_{J, \mathcal{B}}(c)=\{1,2,3\} .
$$

And so $\mathcal{B}$ is a Janet basis for $\langle\mathcal{B}\rangle$ but not a Pommaret basis. In fact $\langle\mathcal{B}\rangle$ has no finite Pommaret basis.

An involutive division, $L$, is called Noetherian if every ideal in $\mathbb{N}_{0}^{n}$ has an involutive basis with respect to $L$. The Janet division is Noetherian while the Pommaret division is not (as seen in the previous example).

Turning to polynomials, let $\mathcal{F}=\left\{f_{1}, \ldots, f_{t}\right\}$ be a finite subset of $\mathcal{P}$ that is head-autoreduced, i.e. no two elements of $\mathcal{F}$ have the same leading term. For an involutive division, $L$, on $\mathbb{N}_{0}^{n}$, we can assign the elements of $\mathcal{F}$ multiplicative variables in $\left\{x^{1}, \ldots, x^{n}\right\}$ in the following, obvious, way. Let $\nu_{i}=\operatorname{le}\left(f_{i}\right)$ and let $\mathcal{B}=\left\{\nu_{1}, \ldots, \nu_{t}\right\}$. Now assign to $f_{i}$ the set of multiplicative variables

$$
X_{L, \mathcal{F}}\left(f_{i}\right)=\left\{x^{j} \mid j \in N_{L, \mathcal{B}}\left(\nu_{i}\right)\right\} \subset\left\{x^{1}, \ldots, x^{n}\right\} .
$$

The subalgebra of $\mathcal{P}$ of polynomials that only involve the variables in $X_{L, \mathcal{F}}\left(f_{i}\right)$ is denoted

$$
\mathbb{k}\left[X_{L, \mathcal{F}}\left(f_{i}\right)\right]
$$


and the set

$$
\mathbb{k}\left[X_{L, \mathcal{F}}\left(f_{i}\right)\right] \cdot f_{i}
$$

is called the involutive span of $f_{i}$. Similarly, the direct sum

$$
\bigoplus_{f_{i} \in \mathcal{F}} \mathbb{k}\left[X_{L, \mathcal{F}}\left(f_{i}\right)\right] \cdot f_{i}
$$

is called the involutive span of $\mathcal{F}$. Notice that if $\mathcal{F}$ happens to be a Gröbner basis for $\mathcal{I}=\langle\mathcal{F}\rangle$ and $\mathcal{B}$ is an involutive basis for $\mathcal{J}=\langle\mathcal{B}\rangle$, then we can write $\mathcal{I}$ as a direct sum

$$
\mathcal{I}=\bigoplus_{f_{i} \in \mathcal{F}} \mathbb{k}\left[X_{L, \mathcal{F}}\left(f_{i}\right)\right] \cdot f_{i}
$$

In this case $\mathcal{F}$ is said to be an involutive basis for $\mathcal{I}$ with respect to the division $L$. The converse also holds; if $(2.3)$ is true then $\mathcal{F}$ is an involutive division, as can easily be checked. We denote the complement of $X_{L, \mathcal{F}}\left(f_{i}\right)$ of non-multiplicative variables by $\bar{X}_{L, \mathcal{F}}\left(f_{i}\right)$. Since the Pommaret division plays a central role in this paper, we give the following definition.

Definition 2.1.4. The set $\mathcal{F} \subset \mathcal{P}$, generating the ideal $\mathcal{I}=\langle\mathcal{F}\rangle$, is said to be involutive if it is involutive with respect to the Pommaret division.

The algorithm that computes the involutive normal form of a polynomial $f$ with respect to a set $\mathcal{H}$ is referred to as $\operatorname{NormalForm}_{L}(f, \mathcal{H})$ and the algorithm that performs an involutive head-autoreduction of a set $\mathcal{H} \subset \mathcal{P}$ with respect to the Pommaret division is referred to as PomHeadAutoReduce $(\mathcal{H})$.

\subsection{Involution algorithms}

The remarkable algorithms that compute involutive bases of polynomial ideals (and some more general types of modules, see [46]) suffer, in the case of the Pommaret division, from the problem of $\delta$-regularity: A given ideal does not have a Pommaret basis in all variables. However, for any ideal $\mathcal{I}$, there always exists a 
change of variables in which $\mathcal{I}$ indeed has a Pommaret basis. The proof of this fact uses the surprising similarity between the Pommaret and the Janet division. The latter division satisfies two important regularity conditions; it is Noetherian and constructive. This guarantees, firstly, the existence of a Janet basis for any ideal and, secondly, allows for an effective algorithm to compute such a basis. The connection between the Pommaret and the Janet division allows for the following strategy for obtaining a Pommaret basis. First calculate a Janet basis using Algorithm 1. This algorithm is the standard algorithm for computing involutive bases for Noetherian and constructive divisions, with a minor modification. In Lines $/ 1 /$ and $/ 9 /$ we perform the involutive autoreductions with respect to the Pommaret basis. The result is still a Janet basis, but it is Pommaret autoreduced. Then we compare the multiplicative variables of the resulting basis w.r.t. both the Pommaret and the Janet division. If all multiplicative variables agree, then we have a simultaneous Pommaret and Janet basis. If they do not agree, then the theory suggests a certain change of variables that is guaranteed to increase the Pommaret span of our Janet basis asymptotically. The algebro-geometric connection of Pommaret bases to the Castelnuovo-Mumford regularity of $\mathcal{I}$ then proves that this procedure will eventually terminate with a Pommaret basis. See [46, Chapters 4-5] for the (again, remarkable) full story. 


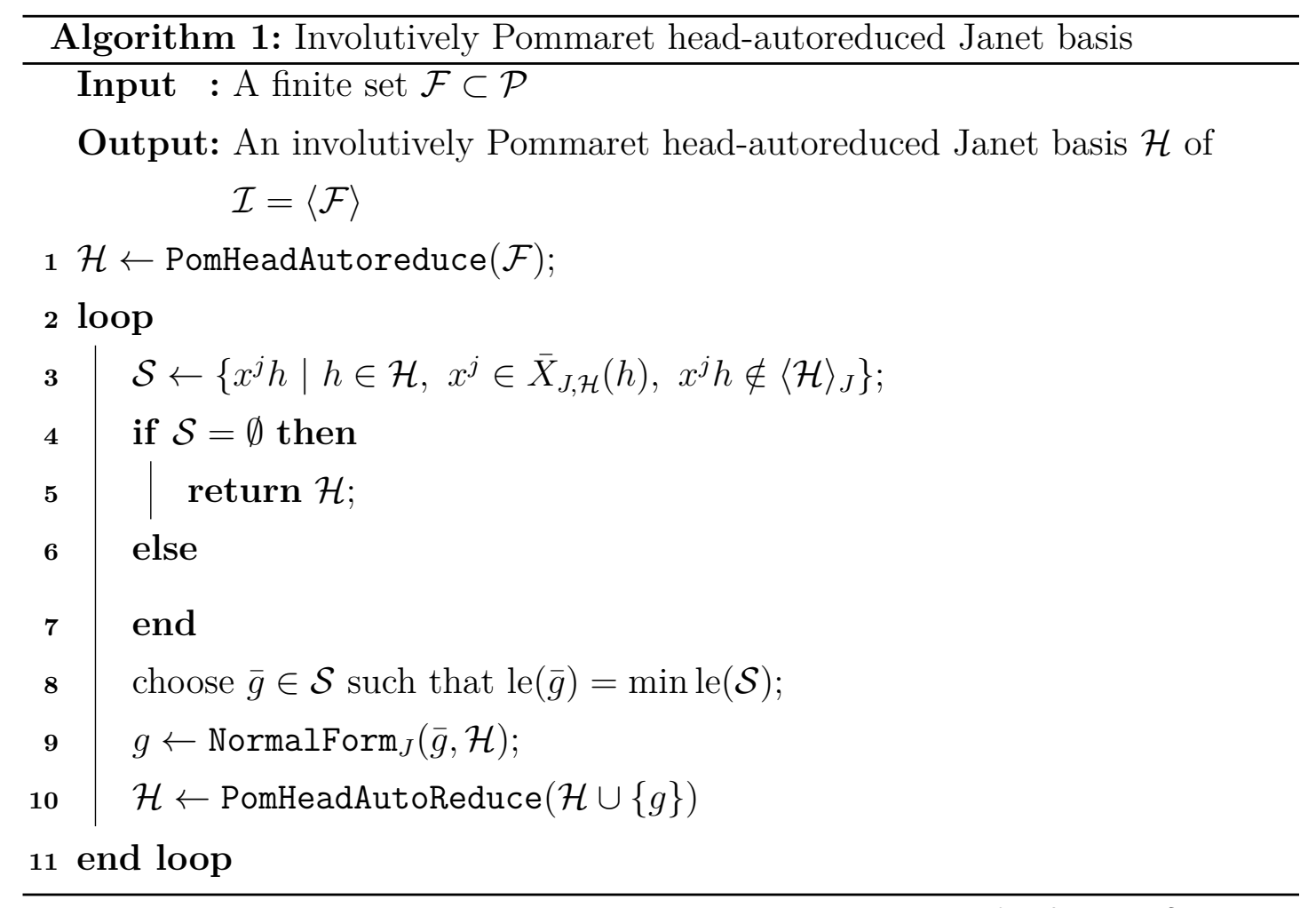

We develop a rather simple, linear algebra-based algorithm for finding $\delta$-regular coordinates. We begin by defining the operator

$$
\mathbf{p r}_{k}(\mathcal{F})=\left\{x^{\nu} f|f \in \mathcal{F},| \nu \mid+\operatorname{deg} f \leq k\right\}
$$

that takes $\mathcal{F}$ and "prolongs" it to degree $k$. For a finite set of polynomials, $\mathcal{F} \subset \mathcal{P}$ we compute a set $\mathcal{F}^{(q+)}$ in the following way. First set $\mathcal{F}^{(q+)}=\mathcal{F}$. Next perform an autoreduction of each set $\pi_{\bar{k}}\left(\mathbf{p r}_{q+1}(\mathcal{F})\right), 1 \leq k \leq q+1$, and add each appearing polynomial of degree $\leq q$ to the set $\mathcal{F}^{(q+)}$. Now repeat this process, starting with $\mathcal{F}^{(q+)}$ instead of $\mathcal{F}$. When we stop getting new polynomials, we terminate the process. In words, we are collecting all polynomials in $\mathcal{I}=\langle\mathcal{F}\rangle$ that have order $\leq q$ and can be obtained by successively applying $\mathbf{p r}_{q+1}$, starting with $\mathcal{F}$. It is easily seen that $\mathcal{F}^{(q+)}$ is a $\mathbb{k}$-linear basis (autoreduction has prevented common leading terms) for the space of polynomials that can be obtained in this way. We 
note that this is, for complicated ideals, in practice, computationally demanding to say the least. However, when completing a differential equation to its involutive form, [46], one is forced to compute all prolongations of the defining equations for the system at hand in the search for integrability conditions. Therefore one must compute the set (2.4) for the differental equation's symbol module anyway! The forthcoming algorithm was designed to apply to Cartan's equivalence method, [14, 36], where the same principle applies; one cannot escape having to compute entire sets of the form (2.4). We give a very simple example elucidating the computation of the sets $\mathcal{F}^{(q+)}$.

Example 2.2.1. Let $\mathcal{F}=\left\{x^{2}+y, x\right\}$ and work with degrevlex with $x<y$. Then $\operatorname{pr}_{2}(\mathcal{F})$ is the set consisting of

$$
x^{2}+y, x^{2}, x y, x
$$

Performing an autoreduction gives the set $\left\{x^{2}, x y, y, x\right\}$ and applying $\mathbf{p r}_{2}$ once more gives $\left\{x, y, x^{2}, x y, y^{2}\right\}$. At this point no more autoreductions are possible. In the language introduced above, we have

$$
\mathcal{F}^{(1+)}=\{x, y\}
$$

Let $\mathcal{I}=\langle\mathcal{F}\rangle$ be an ideal generated by the set $\mathcal{F}=\left\{f_{1}(x), \ldots, f_{t}(x)\right\}$ and make the following (rather strict) assumption on the coordinates $x$. When we run the set $\mathcal{F}$ through Algorithm 1 and find a Pommaret autoreduced Janet basis, for each intermediate set $\mathcal{H}$ obtained along the way (in Line /10/), the Pommaret and Janet multiplicative variables for le $(\mathcal{H})$ agree. We say that such coordinates are super asymptotically regular (or $\sigma$-regular for short) for $\mathcal{F}$ with respect to Algorithm1. This seems a rather luxurious assumption to make but our forthcoming algorithm does not require us to know explicit $\sigma$-regular coordinates; it is enough for us to know that such coordinates always exist, and, in fact, abound as they form a Zariski open set in $G L(n, \mathbb{k})$ being a finite intersection 
of asymptotically regular coordinates (see [46, Theorem 4.3.15]). For $\sigma$-regular coordinates Algorithm 1 will terminate with a Pommaret basis, $\mathcal{H}$, for $\mathcal{I}$, which according to [16] is the unique minimal Janet basis also. Notice that this algorithm expands the original generating set $\mathcal{F}$ to a Pommaret basis by, at each step, looking at "one degree increments" $x^{j} f$ of elements of $\mathcal{F}$ and doing a Pommaret headautoreduction. Let $p+1$ be the maximal degree of all polynomials computed during the determination of the set $\mathcal{S}$ in Line /3/ by Algorithm 1 (this means that the algorithm returns a Pommaret basis of order $p$ ). Since the set $\mathcal{F}^{(p+)}$ is a $\mathbb{k}$-linear basis for the space of all polynomials that can be attained by repeatedly applying $\mathbf{p r}_{p+1}$ to $\mathcal{F}$, and $\mathcal{H}$ is obtained only by applying prolongations from $\mathbf{p r}_{p+1}$, we can write each Pommaret basis element $h \in \mathcal{H}$ uniquely as a $\mathbb{k}$-linear combination

$$
h=\sum_{f \in \mathcal{F}(p+)} c^{f} f, \quad c^{f} \in \mathbb{k} .
$$

This means that there is a unique $f_{h} \in \mathcal{F}^{(p+)}$ with the same leading exponent as $h, \mathrm{le}(h)=\mathrm{le}\left(f_{h}\right)$. Therefore, the subset

$$
\widetilde{\mathcal{H}}=\left\{f_{h}\right\}_{h \in \mathcal{H}} \subset \mathcal{F}^{(p+)}
$$

is a Pommaret basis for $\mathcal{I}$. We should mention that this crude method of obtaining a Pommaret basis is not specific to Algorithm 1, but holds for any Buchberger-type algorithm for computations of Gröbner bases.

Theorem 2.2.2. Let $\mathcal{I}=\langle\mathcal{F}\rangle$ be given in $\sigma$-regular coordinates for $\mathcal{F}$ w.r.t Algorithm 1. If $p$ is the maximal degree of a $\bar{g}$ found in Line /8/ of Algorithm 1 when applied to the set $\mathcal{F}$, then $\mathcal{F}^{(p+)}$ is a weak Pommaret basis for $\mathcal{I}$ and a $\mathbb{k}$-linear basis for $\mathcal{I}^{(\leq p)}=\mathcal{I} \cap \mathcal{P}^{(\leq p)}$.

Proof. We have already proven that $\mathcal{F}^{(p+)}$ is a weak Pommaret basis, since it contains a Pommaret basis. To see that $\mathcal{F}^{(p+)}$ is a $\mathbb{k}$-linear basis for the space of polynomials of degree at most $p$ in $\mathcal{I}$, let $g \in \mathcal{I}^{(\leq p)}$ and define the set $\widetilde{\mathcal{H}}$ as in 2.5 
for $\mathcal{H}$ the Pommaret basis computed by Algorithm 1. We can write

$$
g=\sum_{f \in \widetilde{\mathcal{H}}} P^{f} f, \quad \operatorname{deg} P^{f} f \leq \operatorname{deg} g \leq p
$$

but this trivially implies that $h \in\left\langle\mathcal{F}^{(p+)}\right\rangle_{\mathbb{k}}$ since each $P^{f} f \in\left\langle\mathcal{F}^{(p+)}\right\rangle_{\mathbb{k}}$.

A slightly stronger result is available for homogeneous polynomials. Remember that in $\sigma$-regular coordinates, Algorithm 1 returns the unique minimal Pommaret and Janet basis for $\mathcal{I}$.

Corollary 2.2.3. Let the homogeneous ideal $\mathcal{I}=\langle\mathcal{F}\rangle$ be given in $\sigma$-regular coordinates for $\mathcal{F}$ w.r.t Algorithm 1 . If there exists a $q^{\text {th }}$ degree Pommaret basis for $\mathcal{I}$, then $\mathcal{F}^{(q+)}$ is a weak Pommaret basis for $\mathcal{I}$ and a $\mathbb{k}$-linear basis for $\mathcal{I}^{(\leq q)}$.

Proof. This will follow by Theorem 2.2.2 if we can show that the modified algorithm will only compute $\bar{g}$ with $\operatorname{deg}(\bar{g}) \leq q$. The important difference between the homogeneous and the non-homogeneous cases is that if at some point we have $\operatorname{deg}(\bar{g})=p$ then the normal form in Line $/ 9 /$ is also of degree $p$ if $\mathcal{I}$ is homogeneous. Since this algorithm is sure to find the Pommaret basis of degree $q$ (by uniqueness of a minimal Janet basis), it is clear that all the normal forms $\bar{g}$ also have degree at most $q$.

This proof obviously will not work for non-homogeneous ideals, since then it could happen that a normal form in Line /9/ of Algorithm 1 has much lower degree than its progenitor $\bar{g}$, and could drastically alter the degree of the set $\mathcal{H}$ after autoreduction in Line /10/.

\subsection{Coordinate independent quantities}

Given an ideal $\mathcal{I}=\langle\mathcal{F}\rangle=\left\langle\left\{f_{1}(x), \ldots, f_{t}(x)\right\}\right\rangle$, where deg $\mathcal{F} \leq q$, in coordinates $x$ (not necessarily $\delta$-regular), we wish to find coordinates in which a Pommaret basis exists and to compute it. We know from the results in the last section that 
if $x$ are $\sigma$-regular, and all the $\bar{g}$ computed in Line /8/ in Algorithm 1 have degree $\leq q$ then $\mathcal{F}^{(q+)}$ is a weak Pommaret basis. We begin this section by introducing some coordinate independent quantities that will be helpful.

Let $\mathcal{F}_{q}^{(q+)}$ be the set of $q^{\text {th }}$ degree polynomials in $\mathcal{F}^{(q+)}$, i.e. $\mathcal{F}_{q}^{(q+)}=\pi_{\bar{q}}\left(\mathcal{F}^{(q+)}\right)$, and perform a Pommaret head-autoreduction on the set

$$
\left\{x^{i} \cdot g_{j} \mid 1 \leq i \leq n, g_{j} \in \mathcal{F}_{q}^{(q+)}\right\}
$$

to obtain a set we denote $\mathcal{F}_{q+1}^{(q+)}$. Let $z^{(q+1)}$ be the number of different leading monomials of polynomials of degree exactly $q+1$ in $\mathcal{F}_{q+1}^{(q+)}$ and, dually, let $r^{(q+1)}$ be the number of $q+1$ degree monomials that are not leaders of polynomials from $\mathcal{F}_{q+1}^{(q+)}$. In general we say that a monomial that is not a leader in a set of polynomials is parametric for that set.

Example 2.3.1. Continuing our simple running example, where $\mathcal{F}=\left\{x^{2}+\right.$ $y, x\}$, we had found that $\mathcal{F}^{(1+)}=\{y, x\}$. During the computation of this set we computed the autoreduced set $\mathcal{F}_{2}^{(1+)}=\left\{x^{2}, x y, y^{2}\right\}$ and we have that $z^{(2)}=3$ and $r^{(2)}=0$. Going up one order, we will obviously find that

$$
\mathcal{F}^{(2+)}=\left\{y, x^{2}, x y, y^{2}\right\} \text { and } \mathcal{F}_{3}^{(2+)}=\left\{x^{3}, x^{2} y, x y^{2}, y^{3}\right\}
$$

and so $z^{(3)}=4$ and $r^{(3)}=0$.

Notice that the numbers $z^{(q+1)}$ and $r^{(q+1)}$ are coordinate independent, i.e. we obtain the same numbers if we first perform a linear change of coordinates $x \mapsto \bar{x}$ to obtain $\overline{\mathcal{F}}=\left\{f_{1}(\bar{x}), \ldots, f_{t}(\bar{x})\right\}$ and calculate $\overline{\mathcal{F}}_{q+1}^{(q+)}$. Of course, this is because of the degree preserving algebra-isomorphism between $\mathcal{P}=\mathbb{k}\left[x^{1}, \ldots, x^{n}\right]$ and $\overline{\mathcal{P}}=$ $\mathbb{k}\left[\bar{x}^{1}, \ldots, \bar{x}^{n}\right]$ induced by a change of coordinates. Similarly, the dimension of the space $\left\langle\mathcal{F}^{(q+)}\right\rangle_{\mathbb{k}}$ as a subspace of the $\mathbb{k}_{k}$-linear space $\mathcal{P}^{(\leq q)}$ is coordinate independent.

Example 2.3.2. For the initial set $\mathcal{F}=\left\{x^{2}+y, x\right\}$, consider the change of variables $x=t+s, y=s$. In the new variables we have $\overline{\mathcal{F}}=\left\{(t+s)^{2}+s, t+s\right\}=$ 
$\left\{t^{2}+2 t s+s^{2}+s, t+s\right\}$ and $\mathbf{p r}_{2}(\overline{\mathcal{F}})$ is equal to

$$
\left\{t^{2}+2 t s+s^{2}+s, t^{2}+t s, t s+s^{2}, t+s\right\}
$$

An autoreduction at each fixed order of this set gives

$$
\left\{t, s, t^{2}+t s, t s+s^{2}\right\}
$$

and applying $\mathbf{p r}_{2}$ once more and doing an autoreduction gives $\left\{t, s, t^{2}, t s, s^{2}\right\}$. Therefore we have

$$
\overline{\mathcal{F}}^{(1+)}=\{t, s\} \text { and } \overline{\mathcal{F}}^{(2+)}=\left\{t, s, t^{2}, t s, s^{2}\right\}
$$

which obviously generate isomorphic $\mathbb{k}$-linear spaces to their counterparts in the variables $x$ and $y$, cf. 2.6.

For a set $\mathcal{F}$, in $\sigma$-regular coordinates, of maximal degree $\leq q$, we can test whether or not Algorithm 1 finds a Pommaret basis by only looking at polynomials $\bar{g}$ in Line /8/ that have degree at most $q$, in the following way. If it did, by Theorem 2.2.2, $\mathcal{F}^{(q+)}$ would be a weak Pommaret basis for $\mathcal{I}$. Now, if $x^{\mu}$ is Pommaret multiplicative for $f$ and cls $\mu=i$, then $x^{i}$ is Pommaret multiplicative for $x^{\mu-i} f$. A simple consequence of this property of the Pommaret division is that, if $\mathcal{F}^{(q+)}$ is a weak Pommaret basis, every leader of a polynomial in $\mathcal{I}^{(\overline{q+1})}=\mathcal{I} \cap \mathcal{P}^{(\overline{q+1})}$ is a Pommaret multiplicative product of a polynomial in $\mathcal{F}_{q}^{(q+)}$. Then, the set $\mathcal{F}_{q+1}^{(q+)}$ is actually the same as $\mathcal{I}^{(\overline{q+1})}$. Let $w_{i}^{(q)}$ be the number of leaders in $\mathcal{F}_{q}^{(q+)}$ of class $i$. We then have the equality

$$
z^{(q+1)}=\sum i w_{i}^{(q)}
$$

or, dually, letting $s_{i}^{(q)}$ be the number of parametric monomials of class $i$ for $\mathcal{F}_{q}^{(q+)}$,

$$
r^{(q+1)}=\sum i s_{i}^{(q)} .
$$


Conversely, if, after computing $\mathcal{F}_{q}^{(q+)}$ and $\mathcal{F}_{q+1}^{(q+)}$, we obtain the equality 2.7 then, since local Pommaret bases are global ([46, Chapter 4.1]) and $\mathcal{F}^{(q+)}$ is a $\mathbb{k}$-linear basis for $\mathcal{I}^{(\leq q)}, \mathcal{F}^{(q+)}$ is a weak Pommaret basis for $\mathcal{I}$.

Theorem 2.3.3. Let $\mathcal{I}=\langle\mathcal{F}\rangle=\left\langle f_{1}(x), \ldots, f_{t}(x)\right\rangle$, with $\mathcal{F}$ of maximal degree $\leq q$. Then $\mathcal{F}^{(q+)}$ is a weak Pommaret basis for $\mathcal{I}$ if and only if $r^{(q+1)}=\sum i s_{i}^{(q)}$.

Since the Pommaret span of a set of polynomials is trivially smaller than the ordinary span, we always have the inequality $z^{(q+1)} \geq \sum i w_{i}^{(q)}$, or, dually,

$$
r^{(q+1)} \leq \sum i s_{i}^{(q)}
$$

We call the numbers $s_{i}^{(q)}$ the Cartan characters of degree $q$ for the set $\mathcal{F}_{q}^{(q+)}$. We can test whether there exists a Pommaret basis for $\mathcal{I}$ by checking (2.8) at increasing orders $q$ (note that if $\mathcal{I}$ has a $q^{\text {th }}$ order Pommaret basis then it has a Pommaret basis at all orders above $q$ ). This is guaranteed to eventually work (for $q$ large enough) if we happen to be, serendipitously, in $\sigma$-regular coordinates for our initial generating set. But that is certainly not always the case.

Example 2.3.4. Let $\mathcal{F}=\left\{x^{2}+y, y^{2}\right\}$. An easy computation finds that

$$
\mathcal{F}^{(2+)}=\mathcal{F}
$$

and $r^{(3)}=0$ while $s_{1}^{(2)}=|\{x y\}|=1$ and $s_{2}^{(2)}=0$. Plugging these values into 2.8 gives

$$
r^{(3)}=0<\sum i s_{i}^{(2)}=1 \cdot 1+2 \cdot 0=1,
$$

and the involutivity test fails. However, at the next order, we find that

$$
\mathcal{F}^{(3+)}=\left\{x^{3}+x y, x^{2} y+y^{2}, x y^{2}, y^{3}\right\}
$$

and $r^{(4)}=s_{1}^{(3)}=s_{2}^{(3)}=0$ so the involutivity text is passed and there is a Pommaret basis of order three. Of course, in this simple example, this is all quite obvious 
but we will show below, using Theorem 2.3.3, that the ideal $\mathcal{I}=\langle\mathcal{F}\rangle$ does not have a Pommaret basis of degree two in any variables.

Borrowing the ideas of Cartan from his theory of exterior differential systems in differential geometry, we develop an involutivity test for polynomial ideals. Notice that some of the quantities in Theorem 2.3.3 (namely $\operatorname{dim}_{\mathbb{k}} \pi_{q}\left(\mathcal{F}_{q}^{(q+)}\right)$ and $\left.r^{(q+1)}\right)$ are geometric invariants (i.e. coordinate independent), so maybe there is some hope of developing a coordinate independent version of the test in Theorem 2.3.3. The problem with such a test will then concern the non-invariant numbers $s_{i}^{(q)}$. Notice that $s_{1}^{(q)}$, the number of parametric monomials of degree $q$ for the set $\mathcal{F}_{q}^{(q+)}$ having class 1 , is equal to

$$
\text { the rank of }\left\{x^{1} x^{J}\right\}_{|J|=q-1}
$$

in the $\mathbb{k}$-linear quotient space $\mathcal{P}^{(q)} /\left\langle\pi_{q}\left(\mathcal{F}_{q}^{(q+)}\right)\right\rangle_{\mathbb{k}}$.

Remark 2.3.5. The above argument works because the normal form of a monomial $x^{1} x^{J}$ of class 1 with respect to the set $\mathcal{F}_{q}^{(q+)}$ will still be of class 1 because we are using the degree reverse lexicographic order which is the unique classrespecting order. This term order has an important connection to Pommaret bases and the fact that our algorithm only works for this choice underscores this connection.

Similarly, the number $s_{2}^{(q)}$, of parametric monomials for $\mathcal{F}_{q}^{(q+)}$ having class 2, is the difference of

$$
\text { the rank of }\left\{x^{2} x^{J}\right\}_{|J|=q-1} \cup\left\{x^{1} x^{J}\right\}_{|J|=q-1}
$$

and the previously found $s_{1}^{(q)}$ in the $\mathbb{k}$-linear quotient space $\mathcal{P}^{(q)} /\left\langle\pi_{q}\left(\mathcal{F}_{q}^{(q+)}\right)\right\rangle_{\mathbb{k}}$, and so on.

If we consider the original generating set $\mathcal{F}$ for $\mathcal{I}$ in different variables $\bar{x}=A x$, for some fixed matrix $A$, and calculate $\overline{\mathcal{F}}^{(q+)}$, we obtain the same number of 
parametric monomials of degree $q+1, r^{(q+1)}$. On the other hand, we might very well obtain different numbers, $\bar{s}_{i}^{(q)}$, of parametric monomials of the different classes $i$. Beginning with the number $\bar{s}_{1}^{(q)}$, it is equal to

$$
\text { the rank of }\left\{\bar{x}^{1} \bar{x}^{J}\right\}_{|J|=q-1} \text {, }
$$

in $\overline{\mathcal{P}}^{(q)} /\left\langle\pi_{q} \overline{\mathcal{F}}_{q}^{(q+)}\right\rangle_{\mathbb{k}}$. As before, we view $\overline{\mathcal{P}}^{(q)}$ as a $\mathbb{k}$-linear space with basis consisting of all $\bar{x}^{K}$, with $K \in \mathbb{N}_{0}^{n}$ with $|K|=q$. As mentioned above, a change of variables induces an isomorphism between $\mathcal{P}^{(q)}$ and $\overline{\mathcal{P}}^{(q)}$ and we can write each $\bar{x}^{K}$ as a linear combination of monomials in the $x$ variables:

$$
\bar{x}^{K}=\mathbf{A}_{L}^{K} x^{L},
$$

where $\mathbf{A}$ is a certain invertible matrix whose entries are $|K|^{\text {th }}$ degree polynomials in the entries of the change of variables matrix $A$, where $\bar{x}=A x$. We can therefore view the $x^{L}$ as vectors in $\overline{\mathcal{P}}^{(q)}$, and since $\mathbf{A}$ is invertible, the set $\left\{x^{L}\right\}_{|L|=q}$ is a basis for $\overline{\mathcal{P}}^{(q)}$. With this point of view, see that the elements of the set $\left\{\bar{x}^{1} \bar{x}^{J}\right\}_{|J|=q-1}$ are invertible linear combination of elements from $\left\{\bar{x}^{1} x^{J}\right\}_{|J|=q-1}$. Here, we are writing $\bar{x}^{1} x^{J}$ for the linear combination $\sum a_{j}^{1} x^{j} x^{J}$, where $\bar{x}^{1}=\sum a_{j}^{1} x^{j}$ for $a_{j}^{1}$ in the first line of the change of variables matrix $A$. It is now clear that

$$
\text { the rank of }\left\{\bar{x}^{1} \bar{x}^{J}\right\}_{|J|=q-1}=\text { the rank of }\left\{\bar{x}^{1} x^{J}\right\}_{|J|=q-1} \text {, }
$$

in the $\mathbb{k}_{k}$-linear space $\overline{\mathcal{P}}^{(q)} /\left\langle\pi_{q} \overline{\mathcal{F}}_{q}^{(q+)}\right\rangle_{\mathbb{k}}$. Again, by the isomorphism between $\mathcal{P}^{(q)} \simeq$ $\overline{\mathcal{P}}^{(q)}$, this rank is equal to

$$
\text { the rank of }\left\{a_{j}^{1} x^{j} x^{J}\right\}_{|J|=q-1} \text {, }
$$

in the $\mathbb{k}$-linear quotient space $\mathcal{P}^{(q)} /\left\langle\pi_{q}\left(\mathcal{F}_{q}^{(q+)}\right)\right\rangle_{\mathbb{k}}$ in the original variables.

Note that because of the inequality (2.8) it is desirable to minimize the sum $\sum i \bar{s}_{i}^{(q)}$ over all coordinate changes in the search for $\delta$-regular coordinates. In order 
to achive this, it is at least intuitive to first maximize $\bar{s}_{1}^{(q)}$ by choosing the vector $v^{1}=\left(a_{1}^{1}, \ldots, a_{n}^{1}\right) \in \mathbb{R}^{n}$ such that the rank in $(2.12)$ is maximized. This gives a choice of $\bar{x}^{1}=v^{1} \cdot x$, where $v^{1} \cdot x$ is the Euclidean inner-product of $v^{1}$ and $x$ in $\mathbb{R}^{n}$.

Next, we wish to maximize $\bar{s}_{2}^{(q)}$, that is the number of parametric monomials in $\pi_{q} \mathcal{F}_{q}^{(q+)}$ of class 2 , having already chosen $\bar{x}^{1}$. This means maximizing

$$
\text { the rank of }\left\{\bar{x}^{1} x^{J}\right\}_{|J|=q-1} \cup\left\{a_{j}^{2} x^{j} x^{J}\right\}_{|J|=q-1}
$$

in $\mathcal{P}^{(q)} /\left\langle\pi_{q} \mathcal{F}_{q}^{(q+)}\right\rangle_{\mathbb{k}}$ over all $v^{2}=\left(a_{1}^{2}, \ldots, a_{n}^{2}\right)$. This gives a maximal rank of $\bar{r}_{2}^{(q)}$ and we notice that then $\bar{s}_{2}^{(q)}$ satisfies $\bar{r}_{2}^{(q)}=\bar{s}_{1}^{(q)}+\bar{s}_{2}^{(q)}$. Again, such a $v^{2}$ gives a choice of $\bar{x}^{2}=v^{2} \cdot x$. Let us denote the set $\left\{\bar{x}^{j} x^{J}\right\}_{|J|=q-1}$, where $\bar{x}^{j}=v^{j} \cdot x$, by $\mathcal{C}\left(v^{j}\right)$. At the $j^{\text {th }}$ stage we maximize

$$
\text { the rank of } \mathcal{C}\left(v^{1}\right) \cup \cdots \cup \mathcal{C}\left(v^{j}\right) \text { in } \mathcal{P}^{(q)} /\left\langle\mathcal{F}_{q}^{(q+)}\right\rangle_{\mathbb{k}}
$$

over all $v^{j}$ (having already fixed $v^{1}, \ldots, v^{j-1}$ ) and obtain some number $\bar{r}_{j}^{(q)}$. Then we can see that $\bar{s}_{j}^{(q)}$ satisfies $\bar{s}_{1}^{(q)}+\cdots+\bar{s}_{j}^{(q)}=\bar{r}_{j}^{(q)}$. We continue like this until we have chosen new variables $\bar{x}=\left(v^{1} \cdot x, \ldots, v^{n} \cdot x\right)$.

Remark 2.3.6. Let $r^{(q)}$ be the number of parametric monomials for $\pi_{q} \mathcal{F}_{q}^{(q+)}$ and notice that it is a coordinate independent quantity. Since, in all variables, we have $r^{(q)}=\sum s_{i}^{(q)}$, after having made a choice of $v^{1}, \ldots, v^{n-1}$ and obtained characters $\bar{s}_{1}^{(q)}, \ldots, \bar{s}_{n-1}^{(q)}$, the last character $\bar{s}_{n}^{(q)}$ is strictly determined as $r^{(q)}-\bar{s}_{1}^{(q)}-\cdots-\bar{s}_{n-1}^{(q)}$.

Example 2.3.7. Consider the ideal in $\mathbb{R}[x, y]$ generated by $\mathcal{F}=\{x\}$. As before, we order $x<y$ and use the degrevlex. Obviously $\mathcal{F}^{(1+)}=\{x\}$ and $\bar{s}_{1}^{(1)}$ is the maximal rank of

$$
a x+b y
$$

over all $(a, b) \in \mathbb{R}^{2}$, in the quotient space $\mathcal{P}^{(1)} /\left\langle\mathcal{F}_{1}^{(1+)}\right\rangle_{\mathbb{k}}=\mathcal{P}^{(1)} /\langle x\rangle_{\mathbb{k}}$. Obviously this rank is 1 and is attained for all vectors $(a, b)$ such that $b \neq 0$. Notice that the original coordinates correspond to the choice $(a, b)=(1,0)$ and therefore land in the Zariski closed set in $\mathbb{R}^{2}$ of non $\delta$-regular coordinates. 


\subsection{Reduced Cartan characters}

Unfortunately, and somewhat surprisingly, the above described routine does not minimize $\sum i \bar{s}_{i}^{(q)}$ over all coordinate changes $x \mapsto \bar{x}$. See [36, p. 354] for a counter example. We need to make a slight modification to our routine that, unfortunately, is quite a bit less transparent. At the first stage we maximize (2.12) and obtain the maximal possible number, $\tilde{s}_{1}^{(q)}$, of parametric monomials of class 1 and degree $q$ for $\mathcal{F}_{q}^{(q+)}$ among all coordinates. Say we have obtained $\tilde{s}_{1}^{(q)}, \ldots, \tilde{s}_{j-1}^{(q)}$. At the $j^{\text {th }}$ stage, for $j>1$, we maximize the rank in (2.13) over all $j$-tuples $v^{1}, v^{2}, \ldots, v^{j}$ where we have not fixed $v_{1}, \ldots, v_{j-1}$. This will give some integer $\tilde{r}_{j}^{(q)}$, and we define $\tilde{s}_{j}^{(q)}$ by requiring that $\tilde{s}_{1}^{(q)}+\cdots+\tilde{s}_{j}^{(q)}=\tilde{r}_{j}^{(q)}$. Note that this method does not give an explicit coordinate change, but we prove below that there exists a change of coordinates $x \mapsto \tilde{x}$ such that $\tilde{s}_{i}^{(q)}$ is the number of parametric monomials for $\pi_{q} \mathcal{F}_{q}^{(q+)}$ in the variables $\tilde{x}$. We call the numbers $\tilde{s}_{i}^{(q)}$ the reduced Cartan characters for $\mathcal{F}_{q}^{(q+)}$. For the time being assume that such coordinates exist and notice that the maximal rank $\tilde{r}_{n-1}^{(q)}$ is the maximal possible number of parametric monomials of class $\leq n-1$ over all coordinate changes. Then $\tilde{s}_{n}^{(q)}$ is uniquely determined by Remark 2.3.6 and minimal among all coordinates. This means that in $\tilde{x}$ we have the maximal possible number of leaders of class $n$ in $\mathcal{F}_{q}^{(q+)}$. Let $\tilde{\beta}_{i}^{(q)}$ denote the number of leaders of class $i$ in $\mathcal{F}_{q}^{(q+)}$ in the variables $\tilde{x}$. Similarly, since, in the $\tilde{x}$, we have the maximal possible number of parametric monomials of class $\leq n-2$ (having maximized (2.13) for $j=n-2$ over all possible coordinates $v^{1}, \ldots, v^{n-2}$ ), we have the maximal number of leaders of class $n-1, \tilde{\beta}_{n-1}^{(q)}$ among those coordinates in which there are $\tilde{\beta}_{n}^{(q)}$ leaders of class $n$ in $\mathcal{F}_{q}^{(q+)}$. Iterating this argument we see that, by definition, $\tilde{x}$ are asymptotically regular for the set $\mathcal{F}_{q}^{(q+)}$. Conversely, all asymptotically regular coordinates for $\mathcal{F}_{q}^{(q+)}$ must have the same number, $\tilde{\beta}_{i}^{(q)}$, of leaders of class $i$ as do $\tilde{x}$, and hence also the same Cartan characters $\tilde{s}_{i}^{(q)}$. Since for any given Pommaret head-autoreduced set, asymptotically regular coordinates form a Zariski open (and hence dense) set in $G L(n, \mathbb{k})$, we see that the possible coordinates $\tilde{x}$ are abundant. 
Example 2.4.1. Continuing our analysis of the ideal generated by $\mathcal{F}=\left\{x^{2}+\right.$ $\left.y, y^{2}\right\}$ in Example 2.3.4, let us check for involutivity at order 2 for this ideal. We have $\pi_{2}\left(\mathcal{F}^{(2+)}\right)=\left\{x^{2}, y^{2}\right\}$ and we would like to first maximize the rank of the space generated by

$$
\{(a x+b y) x,(a x+b y) y\}
$$

in $\mathcal{P} / \pi_{2}\left(\mathcal{F}^{(2+)}\right)$. This is easily achieved by computing the normal forms w.r.t. $\pi_{2}\left(\mathcal{F}^{(2+)}\right)$,

$$
\begin{aligned}
(a x+b y) x & \rightarrow b x y \\
(a x+b y) y & \rightarrow a x y .
\end{aligned}
$$

Now, the $\mathbb{k}$-linear space generated by these two normal forms is always one dimensional and so $\tilde{s}_{1}^{(2)}=1$ while $\tilde{s}_{2}^{(2)}=0$. Since $r^{(3)}=0$ we will never satisfy the test in $(2.8)$ in any variables.

Remark 2.4.2. If $\mathcal{F}^{(q+)}$ is a weak Pommaret basis in some variables $x$, then $\operatorname{le}\left(\mathcal{F}_{q}^{(q+)}\right)$ is a Pommaret basis for $\left\langle\operatorname{le}\left(\mathcal{F}_{q}^{(q+)}\right)\right\rangle$ (since $\operatorname{le}\left(\mathcal{F}_{q}^{(q+)}\right)$ is locally involutive) and hence, $x$ are asymptotically regular for $\mathcal{F}_{q}^{(q+)}$. From the discussion above, $\mathcal{F}_{q}^{(q+)}$ must have the Cartan characters $\tilde{s}_{i}^{(q)}$ in the variables $x$, and since $\mathcal{F}^{(q+)}$ is a weak Pommaret basis, we have equality in 2.8

$$
r^{(q+1)}=\sum i \tilde{s}_{i}^{(q)}
$$

We can combine the preceding remark with Theorem 2.3.3 to obtain our fundamental results. But we stress that they only hold for the degree reverse lexicographic order.

Theorem 2.4.3. Let $\mathcal{I}=\langle\mathcal{F}\rangle$ and deg $\mathcal{F} \leq q$. There exist variables in which the set $\mathcal{F}^{(q+)}$ is a weak Pommaret basis if and only if $r^{(q+1)}=\sum i \tilde{s}_{i}^{(q)}$.

Since Algorithm 1 terminates in $\sigma$-regular coordinates, $\mathcal{F}^{(q+)}$ will eventually become a weak Pommaret basis for $\mathcal{I}=\langle\mathcal{F}\rangle$ for large enough $q$ in such coordinates. But this means that we will eventually obtain the equality $r^{(q+1)}=\sum i \tilde{s}_{i}^{(q)}$. 
Theorem 2.4.4. Let $\mathcal{I}=\langle\mathcal{F}\rangle$ and deg $\mathcal{F} \leq q$. An algorithm that successively computes $\mathcal{F}^{(p+)}, r^{(p+1)}$ and $\tilde{s}_{i}^{(p)}$ for $p=q, q+1, q+2, \ldots$, will eventually obtain equality

$$
r^{(p+1)}=\sum i \tilde{s}_{i}^{(p)}
$$

and hence a weak Pommaret basis in some variables $\tilde{x}$.

Remark 2.4.5. Theorem 2.4.4 provides no indication of what is the most efficient way of carrying out the necessary computations of $\mathcal{F}^{(p+)}, r^{(q+1)}$ and $\tilde{s}_{i}^{(p)}$. However, given an initial set $\mathcal{F}$, it is clear that, in more substantial examples than we shall consider here, first computing a Gröbner basis for $\mathcal{I}=\langle\mathcal{F}\rangle$ with respect to the degree reverse lexicographic order will cut down the computational load. This is because the reduced Gröbner basis will have lower order than the Pommaret basis so there is no danger in completing $\mathcal{F}$ to a Gröbner basis first. This will also prevent any new lower order polynomials popping up during the computation of $\mathcal{F}^{(p+)}$ by the defining property of Gröbner bases.

Remark 2.4.6. We note that the above methods and theorems all hold in the more general setting of free modules $\mathcal{P} \times \cdots \times \mathcal{P}=\mathcal{P} \otimes \mathbb{k}^{m}$ with the term over position ordering for the degree reverse lexicographic one. Let $e_{i}$ be the standard basis elements of $\mathbb{k}^{m}$. A typical "monomial" in $\mathcal{P} \otimes \mathbb{k}^{m}$ is then of the form $x^{J} \otimes e_{i}$ and the set of these for all $|J|=q$ is a $\mathbb{k}$-linear basis for $\mathcal{P}^{(q)} \otimes \mathbb{k}^{m}$. Now, let $\mathcal{I} \subset \mathcal{P} \otimes \mathbb{k}^{m}$ be a submodule generated by a set $\mathcal{F} \subset \mathcal{P} \otimes \mathbb{k}^{m}$, the number of parametric monomials of degree $q$ and class 1 for the set $\mathcal{F}_{q}^{(q+)}$ in new variables $\bar{x}$ with $\bar{x}^{1}=\sum a_{j}^{1} x^{j}$ is

$$
\text { the rank of }\left\{\sum a_{j}^{1} x^{j} x^{J} \otimes e_{i}\right\}_{|J|=q-1,1 \leq i \leq m}
$$

in the $\mathbb{k}$-linear space $\left(\mathcal{P}^{(q)} \otimes \mathbb{k}^{m}\right) /\left\langle\pi_{q}\left(\mathcal{F}_{q}^{(q+)}\right)\right\rangle_{\mathbb{k}}$. Here, $\mathcal{F}^{(q+)}$ is defined in the exact same way as in the ideal case. We notice however that in the search for asymptotically regular coordinates, nothing is gained by changing coordinates for the space $\mathbb{k}^{m}$. To see why, notice that such a change will map $e_{i} \mapsto b_{i}^{j} \bar{e}_{j}$ and 
performing elementary row operations will transform the set

$$
\left\{\sum a_{j}^{1} x^{j} x^{J} \otimes b_{i}^{j} \bar{e}_{j}\right\}_{|J|=q-1,1 \leq i \leq m}
$$

back to the set

$$
\left\{\sum a_{j}^{1} x^{j} x^{J} \otimes \bar{e}_{i}\right\}_{|J|=q-1,1 \leq i \leq m}
$$

\subsection{Examples}

Let us finally look at a few simple examples where we implement the above ideas. It remains to be seen whether the ideas presented above can be used to boost the fastest current algorithms that compute Pommaret bases and $\delta$-regular coordinates.

Example 2.5.1. Let $x<y<z$ and as always we use the degrevlex. Let $\mathcal{I}$ be the ideal, in $\mathbb{R}[x, y, z]$, spanned by the set $\mathcal{F}=\left\{f_{1}, f_{2}, f_{3}\right\}:=\left\{\underline{z}^{2}-y^{2}-\right.$ $\left.2 x^{2}, \underline{x z}+x y, \underline{y z}+y^{2}+x^{2}\right\}$. This ideal is an example of one that does not have a Pommaret basis in the chosen variables. Our method detects coordinates in which $\mathcal{I}$ does have a Pommaret basis of degree two and the computations are easy enough to be done by hand. First we need to count the number of parametric monomials of order 3 after one prolongation of the given generating set, i.e. after one application of $\mathbf{p r}_{3}$. We do not have to worry about any new polynomials of order $\leq 2$ appearing since $\mathcal{I}$ is homogeneous and there will be no need to apply $\mathbf{p r}_{3}$ more than once.

It is relatively easy to see that this number is $r^{(3)}=3$. Next, we maximize the rank of

$$
\left\{\tilde{x}^{1} x^{J}\right\}_{|J|=1}=\{\tilde{x} x, \tilde{x} y, \tilde{x} z\}=\{(a x+b y+c z) x,(a x+b y+c z) y,(a x+b y+c z) z\},
$$

modulo the set $\mathcal{F}=\left\{z^{2}-y^{2}-2 x^{2}, x z+x y, y z+y^{2}+x^{2}\right\}$. To set up this calculation, notice that the parametric monomials for $\mathcal{F}$ are $\mathcal{E}:=\left\{x^{2}, x y, y^{2}\right\}$ 
and so each homogeneous second degree polynomial, after subtracting an $\mathbb{R}$-linear combination of elements in $\mathcal{F}$, is an $\mathbb{R}$-linear combination of the elements from $\mathcal{E}$. Our strategy is then to reduce each $\tilde{x}^{1} x^{J}$ in 2.16 to its normal form w.r.t. $\mathcal{F}$ and then viewing the resulting combination of elements of $\mathcal{E}$ as a vector in $\mathbb{R}^{|\mathcal{E}|}=\mathbb{R}^{3}$, the rank of which we can maximize by elementary means. The normal forms of the elements from 2.16 are

$$
\begin{aligned}
& (a x+b y+c z) x \rightarrow a x^{2}+(b-c) x y \equiv(a, b-c, 0) \in \mathbb{R}^{3}, \\
& (a x+b y+c z) y \rightarrow-c x^{2}+a x y+(b-c) y^{2} \equiv(-c, a, b-c) \in \mathbb{R}^{3}, \\
& (a x+b y+c z) z \rightarrow(2 c-b) x^{2}-a x y-(b-c) y^{2} \equiv(2 c-b,-a, c-b) \in \mathbb{R}^{3} .
\end{aligned}
$$

Maximizing the rank of these three vectors in $\mathbb{R}^{3}$ is the same as maximizing the rank of the matrix

$$
\left[\begin{array}{ccc}
a & b-c & 0 \\
-c & a & b-c \\
2 c-b & -a & c-b
\end{array}\right] .
$$

There are many easy choices of $a, b, c$ that maximize this to full rank 3 . One example is $(a, b, c)=(0,1,0)$, i.e. $\tilde{x}=y$, giving the rank 3 matrix

$$
\left[\begin{array}{ccc}
0 & 1 & 0 \\
0 & 0 & 1 \\
-1 & 0 & -1
\end{array}\right]
$$

The first reduced Cartan character is therefore $\tilde{s}_{1}^{(2)}=3$. Next, we maximize the rank of $\mathcal{C}\left(v_{1}\right) \cup \mathcal{C}\left(v_{2}\right)$ in the quotient space $\mathcal{P}^{(2)} /\left\langle\pi_{2}\left(\mathcal{F}_{2}^{(2+)}\right)\right\rangle_{\mathbb{R}}$ but by the normal 
form calculations above this is equivalent to maximizing the rank of the matrix

$$
\left[\begin{array}{ccc}
a & b-c & 0 \\
-c & a & b-c \\
2 c-b & -a & c-b \\
a^{\prime} & b^{\prime}-c^{\prime} & 0 \\
-c^{\prime} & a^{\prime} & b^{\prime}-c^{\prime} \\
2 c^{\prime}-b^{\prime} & -a^{\prime} & c^{\prime}-b^{\prime}
\end{array}\right]
$$

Since we have already seen that $(a, b, c)=(0,1,0)$ maximizes the rank of the first three rows to 3 , and since there are only 3 columns, we must have $\tilde{s}_{2}^{(2)}=0$. Similarly we have $\tilde{s}_{3}^{(2)}=0$. Since $r^{(3)}=3=\sum i \tilde{s}_{i}^{(2)}=1 \cdot 3+2 \cdot 0+3 \cdot 0$, Cartan's test is successful for any choice of $\tilde{y}$ and $\tilde{z}$, as long as the linear change of variables is invertible. Arbitrarily, we choose $\tilde{y}=x$ and $\tilde{z}=z$. That is, we have just switched $x$ and $y$. The generating set $\mathcal{F}$ in the new variables is (again, we underline degrevlex leading terms)

$$
\tilde{\mathcal{F}}=\left\{\underline{\tilde{z}^{2}}-\tilde{x}^{2}-2 \tilde{y}^{2}, \underline{\tilde{y}} \tilde{z}+\tilde{x} \tilde{y}, \tilde{x} \tilde{z}+\tilde{x}^{2}+\underline{\tilde{y}^{2}}\right\}
$$

which is easily seen to be involutive. The reduced Cartan characters $\tilde{s}_{1}^{(2)}=3, \tilde{s}_{2}^{(2)}=$ 0 and $\tilde{s}_{3}^{(2)}=0$ tell us the classes of the parametric monomials of degree 2 , and we can indeed check that these are

$$
\tilde{x}^{2}, \tilde{x} \tilde{y}, \tilde{x} \tilde{z},
$$

i.e. three of class 1 , and none of class 2 and 3 .

Notice that setting $(a, b, c)=(1,0,0)$ in the matrix 2.17) would give the suboptimal rank 2 , which is symptomatic of the non $\delta$-regularity of the original variables $x, y, z$.

We have seen how our methods simplify in the homogeneous case. As expected, further simplifications arise in the monomial case. 
Example 2.5.2. Another example of an ideal not having a finite Pommaret basis in the given variables is $\mathcal{I}=\langle\mathcal{F}\rangle=\left\langle x^{2} z, x y, x z^{2}\right\rangle$. We calculate $\mathbf{p r}_{4} \mathcal{F}$ and find that $r^{(4)}=6$. The parametric monomials for $\mathcal{F}_{3}^{(3+)}$ are

$$
\mathcal{E}_{3}=\left\{x^{3}, y^{3}, y^{2} z, y z^{2}, z^{3}\right\}, \quad|\mathcal{E}|=5,
$$

and they will correspond to the columns of our matrices. Computing normal forms of the elements in the set $\left\{\tilde{x}^{1} x^{J}\right\}_{|J|=2}$ w.r.t. $\mathcal{F}_{3}^{(3+)}$ gives $\left(\right.$ for $\left.\tilde{x}^{1}=a x+b y+c z\right)$

$$
\left\{\tilde{x}^{1} x^{J}\right\}_{|J|=2} \rightarrow\left\{a x^{3}, b y^{3}+c y^{2} z, b y^{2} z+c y z^{2}, b y z^{2}+c z^{3}\right\} .
$$

Setting up the corresponding matrix gives

$$
\left[\begin{array}{lllll}
a & 0 & 0 & 0 & 0 \\
0 & b & c & 0 & 0 \\
0 & 0 & b & c & 0 \\
0 & 0 & 0 & b & c
\end{array}\right] .
$$

We obtain maximal rank $\tilde{s}_{1}^{(3)}=4$ by choosing, for example, $(a, b, c)=(1,1,0)$. Notice that the given coordinates correspond to the choice $(a, b, c)=(1,0,0)$, giving the far from optimal rank 1 . At the next step, we need to maximize the rank of

$$
\left[\begin{array}{ccccc}
a & 0 & 0 & 0 & 0 \\
0 & b & c & 0 & 0 \\
0 & 0 & b & c & 0 \\
0 & 0 & 0 & b & c \\
a^{\prime} & 0 & 0 & 0 & 0 \\
0 & b^{\prime} & c^{\prime} & 0 & 0 \\
0 & 0 & b^{\prime} & c^{\prime} & 0 \\
0 & 0 & 0 & b^{\prime} & c^{\prime}
\end{array}\right]
$$

over all choices of $(a, b, c),\left(a^{\prime}, b^{\prime}, c^{\prime}\right) \in \mathbb{R}^{3}$. The vectors $(a, b, c)=(1,1,0)$ and 
$\left(a^{\prime}, b^{\prime}, c^{\prime}\right)=(0,0,1)$ will do, giving the matrix 2.20 full rank 5 , and the reduced character $\tilde{s}_{2}^{(3)}=5-\tilde{s}_{1}^{(3)}=1$. Before we perform the calculation for $\tilde{s}_{3}^{(3)}$, which will entail placing a copy of the matrix (2.19), with entries depending on a third vector $\left(a^{\prime \prime}, b^{\prime \prime}, c^{\prime \prime}\right)$ underneath the matrix $(2.20)$ and maximizing the rank, we notice that since we have already attained full column rank of 2.20 we will find that $\tilde{s}_{3}^{(3)}=0$. Now we check for involution using Cartan's test, but we find that

$$
r^{(4)}=6=\sum i \tilde{s}_{i}^{(3)}=1 \cdot 4+2 \cdot 1+3 \cdot 0=6
$$

and so there does exist a Pommaret basis of degree 3 after making the change of variables $\tilde{x}=x+y, \tilde{y}=z$ and $\tilde{z}=y$. In the new variables the ideal becomes $\mathcal{I}=\left\langle x^{2} z, x y, x z^{2}\right\rangle \mapsto\left\langle(\tilde{x}-\tilde{z})^{2} \tilde{y},(\tilde{x}-\tilde{z}) \tilde{z},(\tilde{x}-\tilde{z}) \tilde{y}^{2}\right\rangle=\left\langle\tilde{x} \tilde{y} \tilde{z}-\tilde{x}^{2} \tilde{y}, \underline{\tilde{z}}^{2}-\tilde{x} \tilde{z}, \tilde{y}^{2} \tilde{z}-\tilde{x} \tilde{y}^{2}\right\rangle$ (after a little bit of autoreduction). As is easily checked, this generating set is indeed a Pommaret basis for $\mathcal{I}$. 


\section{Chapter 3}

\section{Cartan-Kuranishi completion}

We can apply our results from the last chapter to the problem of completing a differential equation to its involutive form, see [46]. Here, and in the rest of the thesis, all diffeomorphisms, differential equations and maps are assumed realanalytic. This is necessary since we need the Cartan-Kähler theorem to guarantee local solvability of (well behaved) formally integrable equations, which requires analyticity but all our constructions otherwise work in the smooth category. Let $\mathcal{E}$ be the trivial bundle $\mathbb{R}^{n} \times \mathbb{R}^{m} \rightarrow \mathbb{R}^{n}$ in coordinates $x \in \mathbb{R}^{n}, u \in \mathbb{R}^{m}$, and let $J^{q}(\mathcal{E})$ be the space of $q$-jets of sections of $\mathcal{E}$ for $0 \leq q \leq \infty$. We denote the elements of $J^{q}(\mathcal{E})$ by $j^{q} u_{x}$ or $\left(x, u^{(q)}\right)$.

Consider a $q^{\text {th }}$ order differential equation on $\mathcal{E}$,

$$
F\left(x, u^{(q)}\right)=0 .
$$

We denote the set of points in the $q^{\text {th }}$ order jet space that satisfy the equation (3.1) by $\mathcal{R}_{q} \subset J^{q}(\mathcal{E})$.

Remark 3.0.1. We shall refer both to the equations 3.1) and the subset $\mathcal{R}_{q} \subset$ $J^{p}(\mathcal{E})$ that they determine as a differential equation.

We can prolong the set (3.1) of equations to order $q+1$ by adjoining to 3.1) 
all the equations

$$
D_{1} F=0, \ldots, D_{n} F=0
$$

and obtain the set $\mathcal{R}_{q, 1} \subset J^{q+1}(\mathcal{E})$, where $D_{i}$ is the total derivative operator on $J^{\infty}(\mathcal{E})$,

$$
D_{i}=\frac{\partial}{\partial x^{i}}+\sum_{|J| \geq 0} u_{J, i}^{\alpha} \frac{\partial}{\partial u_{J}^{\alpha}} .
$$

Note that every local solution to 3.1 must also satisfy the prolonged equation. An integrability condition appearing when going from $\mathcal{R}_{q}$ to $\mathcal{R}_{q, 1}$ is an equation of order at most $q$ that is an algebraic consequence of the equations (3.2) but not an algebraic consequence of the equations (3.1). That is, it is a new equation of order (at most) $q$ that solutions to (3.1) must satisfy and should be added to (3.1). The set of points in $\mathcal{R}_{q}$ that also satisfy these integrability conditions is denoted $\mathcal{R}_{q, 1}^{(1)}$. We can describe the set $\mathcal{R}_{q, 1}^{(1)}$ using the canonical projections $\pi_{t}^{p}: J^{p}(\mathcal{E}) \rightarrow J^{t}(\mathcal{E})$, $0 \leq t \leq p \leq \infty$, between the jet spaces:

$$
\mathcal{R}_{q, 1}^{(1)}=\pi_{q}^{q+1}\left(\mathcal{R}_{q, 1}\right)
$$

Note that the presence of integrability conditions is equivalent to the condition that $\mathcal{R}_{q, 1}^{(1)} \subsetneq \mathcal{R}_{q}$.

More generally, adjoining all prolongations of 3.1 of order $t, D_{J} F=0, J \in \mathbb{N}_{0}^{n}$ with $|J|=t$, we arrive at the set $\mathcal{R}_{q, t} \subset J^{q+t}(\mathcal{E})$. We denote the projection $\pi_{q+t-s}^{q+t}\left(\mathcal{R}_{q, t}\right)$ by $\mathcal{R}_{q, t}^{(s)} \subset \mathcal{R}_{q, t-s}$. The differential equation $\mathcal{R}_{q}$ is formally integrable if $\mathcal{R}_{q, t}^{(s)}=\mathcal{R}_{q, t-s}$ for all $t \geq s$

For a $q^{\text {th }}$ order equation $\mathcal{R}_{q} \subset J^{q}(\mathcal{E})$, given by a system 3.1 , we shall write $\mathcal{R}_{\infty}$ for the set of points in $J^{\infty}(\mathcal{E})$ that satisfy 3.1 and all its prolongations (and hence all integrability conditions of all orders).

Remark 3.0.2. To prevent too much degeneracy in our differential equations, we assume that for all differential equations we encounter that the full system $\mathcal{R}_{\infty} \subset J^{\infty}(\mathcal{E})$ is fibered over all of $\mathbb{R}^{n}$, i.e. the system does not impose any restrictions on the independent variables alone. We shall refer to such differential 
equations as genuine differential equations.

Remark 3.0.3. If $\mathcal{R}_{q}$ is given by equations (3.1) and some equations are of order strictly less that $q$ we "complete" (3.1) to an equivalent $q^{\text {th }}$ order system in the following way. Let $\mathcal{S}_{q-1} \subset J^{q-1}(\mathcal{E})$ be the system determined by the equations in (3.1) of order strictly less that $q$. Prolong all these equations to order $q$ to obtain $\mathcal{S}_{q}$ and replace $\mathcal{R}_{q}$ by $\mathcal{S}_{q} \cap \mathcal{R}_{q}$. Now repeat this process, prolonging each equation of order $<q$ to order $q$, and so forth. This process generates a decreasing sequence of subspaces of $J^{q}(\mathcal{E})$. Our regularity assumptions for the differential equations we consider (cf. Remark 3.0.5) will guarantee that this sequence terminates at a $q^{\text {th }}$ order system. This new system has an important property; if $F_{j}\left(x, u^{(q-s)}\right)=0$ is an equation in $\mathcal{S}_{q} \cap \mathcal{R}_{q}$, then any prolongation of it, $D_{J} F_{j}=0,|J| \leq s$, appears as an equation in $\mathcal{S}_{q} \cap \mathcal{R}_{q}$. Assume $\mathcal{R}_{q}$ is complete in the above way and consider a point $\left(x_{0}, u_{0}^{(q)}\right) \in \mathcal{R}_{q}$ and let $\varphi$ be a local solution to the equations of order exactly $q$ only, but such that $j^{q} \varphi_{x_{0}}=\left(x_{0}, u_{0}^{(q)}\right)$. Then we have, for any equation $F_{j}\left(x, u^{(q-1)}\right)=0$ in $\mathcal{R}_{q}$ of order $q-1$ that, for all $i$,

$$
D_{i}\left(F_{j}\left(j^{q-1} \varphi_{\mid x}\right)\right)=0 \quad \text { and } \quad F_{j}\left(j^{q-1} \varphi_{\left.\right|_{x_{0}}}\right)=0
$$

But this means that $\varphi$ is a local solution to all equations in $\mathcal{R}_{q}$ of order $q-1$, $F_{j}\left(j^{(q-1)} \varphi_{x}\right)=0$. Similarly, $\varphi$ is a local solution to all the lower order equations in $\mathcal{R}_{q}$. This means that for questions of local solvability it is sufficient to consider systems $\mathcal{R}_{q}$ that are determined by equations of order exactly $q$ only. We assume all differential equations $\mathcal{R}_{q}$ have been completed in this way and by the symbol of $\mathcal{R}_{q}$ we mean the symbol of all equations of top order $q$.

Remark 3.0.4. We should mention that the issues alluded to in the last remark only pose a problem globally. Locally, each equation $\Phi\left(x, u^{(q)}\right)=0$ can be put in solved form,

$$
u_{J}^{\alpha}=\Psi\left(x, u^{(q)}\right),
$$

and restricting to the (dense, open) subset of $\mathcal{R}_{q}$ where it, and all the systems 
in the decreasing sequence constructed in Remark 3.0.3, can be written in solved form obviously bypasses any issues of termination of this sequence.

Remark 3.0.5. Let $\mathcal{R}_{q}$ be a differential equation and let $j^{\infty} u_{\left.\right|_{x}} \in \mathcal{R}_{\infty}$. An integrability condition appearing in $\mathcal{R}_{q, t}^{(s)} \subsetneq \mathcal{R}_{q, t-s}$ enlarges the symbol module of the original $\mathcal{R}_{q}$ at $\pi_{q}^{\infty}\left(j^{\infty} u_{\left.\right|_{x}}\right)$ and by the Hilbert basis theorem we will eventually stop finding such integrability conditions and the symbol module eventually stabilizes at each point. To make sure that this happens at the same time for all points in $\mathcal{R}_{\infty}$ we make the following assumption on all equations in this thesis: For all $s \leq q+t, \mathcal{R}_{q, t}^{(s)}$ is a submanifold in $J^{q+t-s}(\mathcal{E})$ and there is a $p^{*}$, called the regularity order of $\mathcal{R}_{q}$, such that the fibers of

$$
\mathcal{R}_{q, t}^{(s)} \rightarrow \pi_{p^{*}}^{q+t-s}\left(\mathcal{R}_{q, t}^{(s)}\right)
$$

have constant dimension for all $s, t$ such that $q+t-s \geq p^{*}$. This will prevent the degeneracy mentioned above since above the regularity order the symbol modules have the same homogeneous dimensions at different points in the differential equation.

This will also guarantee that the completion process of a differential equation to some order $q$ from Remark 3.0 .3 will terminate with a system with the desired properties as long as $q$ is at least as large as the regularity order.

Definition 3.0.6. We say that $\mathcal{R}_{q}$ is regular if it satisfies the blanket assumption from the previous remark (for some regularity order $p^{*}$ ) and for all $s, t$ such that $q+t-s \geq p^{*}$ the symbol of $\mathcal{R}_{q, t}^{(s)}$ has constant reduced Cartan characters.

This definition is subtle and has important consequences; we shall prove in Section 3.3 that it is equivalent to local solvability (modulo integrability conditions) and it will be important when we tackle the thorny issue of tameness of Lie pseudo-groups in the appendix.

Example 3.0.7. Consider the second order system, $\mathcal{R}_{2}$, of differential equations 
for maps

$$
(x, y, u) \mapsto(X(x, y, u), Y(x, y, u), U(x, y, u))
$$

given by the first order equations

$$
\begin{gathered}
X=x, \quad Y=y, \quad U=u+x U_{x}+y U_{y}, \\
X_{x}=Y_{y}=U_{u}=1, \quad X_{y}=X_{u}=Y_{x}=Y_{u}=0
\end{gathered}
$$

and the trivial second order equations

$$
X_{i j}=Y_{i j}=U_{i j}=0, \quad \text { for all } i, j \in\{x, y, u\} .
$$

Obviously $\mathcal{R}_{2}^{(1)}$, given by the equations 3.3 , is a manifold but the symbol of $\mathcal{R}_{2}^{(1)}$ is non regular since for $x=y=0$ the equation

$$
U=u+x U_{x}+y U_{y}
$$

drops in degree. However, above the regularity order $p^{*}=2$ the symbol module is uniform and so $\mathcal{R}_{2}$ is regular in the sense of Definition 3.0.6. Since $\mathcal{R}_{2}$ is formally integrable it is locally solvable by our forthcoming results (in this simple example, local solvability is obvious). We shall come back to this example in the appendix when we discuss tameness of Lie pseudo-groups.

Definition 3.0.8. A formally integrable differential equation $\mathcal{R}_{q} \subset J^{\infty}(\mathcal{E})$ is involutive if it has constant reduced Cartan characters and its symbol is involutive at all points in $\mathcal{R}_{q}$.

Remark 3.0.9. For regular differential equations, possibly after some prolongation/projection, involution of the symbol at a point implies involution of the symbol in an open neighborhood. We shall prove, in Section 3.3, that regular equations can, again, possibly after some prolongation/projection, be written in Cartan normal form, [46, p. 288], and therefore the Cartan-Kähler theorem applies to obtain the structure of the local solutions. Better yet, this concept of regularity 
is essentially equivalent to being able to write the system in Cartan normal form, thereby characterizing such equations. The motivation for our algorithm came from the theory of Lie pseudo-groups, and we shall prove, in Appendix A, that all Lie pseudo-groups are regular on a dense, open and invariant set.

At first sight, formal integrability (and involutivity) looks like a condition that can only be affirmed by checking all prolongations of $\mathcal{R}_{q}$, but fortunately there exists a finite process for obtaining a formally integrable equation from the initial set $\mathcal{R}_{q}$ if $\mathcal{R}_{q}$ is regular. The following theorem is the key (see [46]).

Theorem 3.0.10. Let $\mathcal{R}_{q}$ be a $q^{\text {th }}$ order, regular, differential equation whose symbol is involutive and assume that $\mathcal{R}_{q, 1}^{(1)}=\mathcal{R}_{q}$, then $\mathcal{R}_{q}$ is formally integrable.

\subsection{Completion for formally integrable equations}

Assume, for simplicity, that $\mathcal{R}:=\mathcal{R}_{\infty} \subset J^{\infty}(\mathcal{E})$ is a formally integrable differential equation given by a collection of equations

$$
F\left(x, u^{(\infty)}\right)=0
$$

delaying the discussion of completing general PDEs to involution until the next subsection.

Denote the standard contact forms on $J^{\infty}(\mathcal{E})$ by $\Upsilon_{J}^{\alpha}:=d u_{J}^{\alpha}-u_{J, i}^{\alpha} d x^{i}, J \in \mathbb{N}_{0}^{n}$. When we restrict the contact co-distribution on $J^{\infty}(\mathcal{E})$ to $\mathcal{R}$ we get certain linear dependencies among the $\Upsilon_{J}^{\alpha}$ according to

$$
0=d F=d_{H} F+d_{V} F=D_{i} F d x^{i}+\frac{\partial F}{\partial u_{J}^{\alpha}} \Upsilon_{J}^{\alpha}=\frac{\partial F}{\partial u_{J}^{\alpha}} \Upsilon_{J}^{\alpha},
$$

where, $d_{H}$ is the horizontal component of $d, D_{i}$ is the total derivative w.r.t. $x^{i}$, $d_{V}$ is the contact component of the exterior derivative on $J^{\infty}(\mathcal{E})$ and $F$ is the collection of defining equations of $\mathcal{R}$. The terms $D_{i} F$ vanish as they are among 
the defining equations of $\mathcal{R}$. Note the Einstein summation convention, which will be employed whenever possible.

The symbol module of a differential equation, $\mathcal{R} \subset J^{\infty}(\mathcal{E})$, at each point in $\mathcal{R}$, transforms, under a change of variables, like a submodule of $\mathcal{P} \otimes \mathbb{R}^{m}$. We can then test whether there exist change of variables that make the symbol of $\mathcal{R}$ involutive, at some order $q$, by directly applying our results from the last chapter to the symbol of $\mathcal{R}_{q}=\pi_{q}^{\infty}(\mathcal{R})$. However, to prove termination of Cartan's equivalence method we develop an equivalent method that focuses on the exterior derivative and contact forms.

Let $\Gamma=\oplus_{q \geq 0} \Gamma(q)$ denote the vector space generated by the one-forms $\Upsilon_{J}^{\alpha}$, and $\Gamma(q)$ by those of order $q$, i.e. the $\Upsilon_{J}^{\alpha}$ with $|J|=q$. Assume that we assign principal and parametric derivatives of $\mathcal{R}$ according to degrevlex. We have $d \Upsilon_{J}^{\alpha}=d x^{i} \wedge \Upsilon_{J, i}^{\alpha}$, so a straightforward way of counting parametric derivatives of class 1 and order $q$ is to calculate the rank, $s_{1}^{(q)}$, of the collection

$$
\left.\left\{\gamma_{q}\left(D_{1}\right\lrcorner d \Upsilon_{J}^{\alpha}\right)_{\left.\right|_{\mathcal{R}}}\right\}_{|J|=q-1,1 \leq \alpha \leq m}=\left\{\Upsilon_{J, 1 \mid \mathcal{R}}^{\alpha}\right\}_{|J|=q-1,1 \leq \alpha \leq m},
$$

where $\gamma_{q}$ is the projection $\Gamma \rightarrow \Gamma(q)$. This works since we are using degrevlex, so only contact forms of class 1 appear in the collection (3.6). In the following, to keep the notation simple, we shall simply write the subscript $q-1$ instead of the cumbersome $|J|=q-1,1 \leq \alpha \leq m$. Next, to count parametric derivatives of class 2 and order $q, s_{2}^{(q)}$, we can find the rank, $r_{2}^{(q)}$, of the collection

$$
\left.\left.\left\{\gamma_{q}\left(D_{2}\right\lrcorner d \Upsilon_{J}^{\alpha}\right)_{\left.\right|_{\mathcal{R}}}\right\}_{q-1} \cup\left\{\gamma_{q}\left(D_{1}\right\lrcorner d \Upsilon_{J}^{\alpha}\right)_{\left.\right|_{\mathcal{R}}}\right\}_{q-1}=\left\{\Upsilon_{J,\left.2\right|_{\mathcal{R}}}^{\alpha}\right\}_{q-1} \cup\left\{\Upsilon_{J,\left.1\right|_{\mathcal{R}}}^{\alpha}\right\}_{q-1},
$$

in the $\mathbb{R}$-linear space $\gamma_{q}\left(\Gamma(q)_{\left.\right|_{\mathcal{R}}}\right)$. Then $s_{1}^{(q)}+s_{2}^{(q)}=r_{2}^{(q)}$. We can continue in this way to find all the Cartan characters $s_{1}^{(q)}, \ldots, s_{n}^{(q)}$.

Now, what if we change coordinates $(x, u) \stackrel{f}{\mapsto}(\bar{x}, \bar{u})$ ? First of all, by Remark 2.4.6, when computing the reduced Cartan characters for the symbol module at some degree, there is nothing to be gained from changing dependent variables $u \mapsto$ $\bar{u}$. We therefore restrict to a change of independent variable $x \stackrel{f}{\mapsto} \bar{x}$. Denote the 
standard contact one-forms in the new variables by $\bar{\Upsilon}_{K}^{\alpha}$. The change of variables $f$ (or rather, its infinite prolongation) pulls these contact forms in the $\bar{x}$ variables to contact forms in the $x$ variables, that is

$$
f^{*} \bar{\Upsilon}_{K}^{\alpha}=\mathbf{A}_{K}^{J} \Upsilon_{J}^{\alpha}
$$

where the $\mathbf{A}_{K}^{J}$ are functions of jets of $f$ of order at most $|K|$ and the $\Upsilon_{J}^{\alpha}$ have $|J| \leq|K|$. Fixing some order $q-1$, the matrix with $\mathbf{A}_{K}^{J}$ in entry $(J, K)$ for all $|J|=|K|=q-1$ is invertible, and coincides with the matrix from (2.10) at each point in the domain of $f$ if we set $\bar{x}=\nabla f \cdot x$ (where $\nabla f$ denotes the Jacobian matrix of $f$ ).

For the above described routine for counting the number of parametric derivatives of certain classes, instead of working with the $\Upsilon_{J}^{\alpha}$ with $|J|=q-1$, we will get the same answers if we instead work with the linear combinations

$$
\Psi_{K}^{\alpha}:=\sum_{|J|=q-1} \mathbf{A}_{K}^{J} \Upsilon_{J}^{\alpha}, \quad|K|=q-1,1 \leq \alpha \leq m
$$

That is, the number of parametric derivatives of class 1 is equal to the rank of the collection $\left.\left\{\gamma_{q}\left(D_{1}\right\lrcorner d \Psi_{K}^{\alpha}\right)_{\left.\right|_{\mathcal{R}}}\right\}_{q-1}$ and so on. In the barred variables, the number of parametric derivatives of class 1 is the rank of $\left.\bar{\gamma}_{q}\left(\bar{D}_{1}\right\lrcorner \bar{\Upsilon}_{K}^{\alpha}\right)\left.\right|_{\mathcal{R}}$, where $\bar{\gamma}_{q}$ is the obvious operator in the barred variables. Assume $f_{*}\left(a_{1}^{i} D_{i}\right)=\bar{D}_{1}$ at some point and note that, since $f$ is induces, at each order $q$, a diffeomorphism from $\mathcal{R}_{q}$ to $\mathcal{R}_{q}$ in the barred variables,

$$
\begin{aligned}
\operatorname{rank}\left\{\bar{\gamma}_{q}\left(\bar{D}_{1}\right\lrcorner d \bar{\Upsilon}_{K}^{\alpha}\right)_{\left.\left.\right|_{\mathcal{R}}\right\}_{q-1}} & \left.=\operatorname{rank}\left\{\gamma_{q} f^{*}\left(\bar{D}_{1}\right\lrcorner d \bar{\Upsilon}_{K}^{\alpha}\right)_{\left.\right|_{\mathcal{R}}}\right\}_{q-1} \\
& \left.=\operatorname{rank}\left\{\gamma_{q}\left(a_{1}^{i} D_{i}\right\lrcorner d f^{*} \bar{\Upsilon}_{K}^{\alpha}\right)_{\left.\right|_{\mathcal{R}}}\right\}_{q-1} \\
& \left.=\left.\operatorname{rank}\left\{\gamma_{q}\left(a_{1}^{i} D_{i}\right\lrcorner d\left(\mathbf{A}_{K}^{J} \Upsilon_{J}^{\alpha}\right)\right)\right|_{\mathcal{R}}\right\}_{q-1} \\
& \left.=\operatorname{rank}\left\{\gamma_{q}\left(a_{1}^{i} D_{i}\right\lrcorner d \Upsilon_{J}^{\alpha}\right)_{\left.\right|_{\mathcal{R}}}\right\}_{q-1} .
\end{aligned}
$$

In the last equality we have used the fact that $\mathbf{A}_{K}^{J}$ depends only on jets of order 
$q-1$ and the fact mentioned above that we can take invertible linear combinations $\mathbf{A}_{K}^{J} \Upsilon_{J}^{\alpha}$ without affecting the ranks.

In general, if $f_{*}\left(a_{j}^{i} D_{i}\right)=\bar{D}_{j}, 1 \leq j \leq n$, at some point in $\mathcal{R}_{q}$, we have

$$
\left.\left.\operatorname{rank}\left\{\bar{\gamma}_{q}\left(\bar{D}_{j}\right\lrcorner d \bar{\Upsilon}_{K}^{\alpha}\right)_{\left.\right|_{\mathcal{R}}}\right\}_{q-1}=\operatorname{rank}\left\{\gamma_{q}\left(a_{j}^{i} D_{i}\right\lrcorner d \Upsilon_{J}^{\alpha}\right)_{\left.\right|_{\mathcal{R}}}\right\}_{q-1}
$$

We will now use (3.7) to devise an algorithm that tests for involutivity of $\mathcal{R}$ at some fixed order $q$. Denote the vectors $\left(a_{j}^{1}, a_{j}^{2}, \ldots, a_{j}^{n}\right)$ by $v_{j}$ and define the set of one-forms $\left.\mathcal{C}\left(v_{j}\right):=\left\{\gamma_{q}\left(a_{j}^{i} D_{i}\right\lrcorner d \Upsilon_{J}^{\alpha}\right)_{\left.\right|_{\mathcal{R}}}\right\}_{q-1}$ at each point in $\mathcal{R}$. Let $r^{(q+1)}$ be the number of parametric derivatives of order $q+1$. We can calculate the reduced Cartan characters of the symbol module of $\mathcal{R}$ at a point $\left(x_{0}, u_{0}^{(\infty)}\right) \in \mathcal{R}$ by first maximizing the rank of $\mathcal{C}\left(v_{1}\right)$ to obtain $\tilde{s}_{1}^{(q)}$. Next maximize the rank of $\mathcal{C}\left(v_{1}\right) \cup \mathcal{C}\left(v_{2}\right)$ and set it equal to $\tilde{s}_{1}^{(q)}+\tilde{s}_{2}^{(q)}$, and so on. By the arguments from Section 2.4, there is an invertible matrix $A$, with rows $v_{j}$, for which the sets $\mathcal{C}\left(v_{1}\right) \cup \cdots \cup \mathcal{C}\left(v_{j}\right)$ obtain their maximal ranks for all $1 \leq j \leq n$. Make a change of variables $x \mapsto \tilde{x}=f(x)$, with $f\left(x_{0}\right)=\tilde{x}_{0}$, where the Jacobian of $f$ at the point $x_{0}$ is equal to $A$ (the linear change of variables $\tilde{x}=A x$ will do). Then, in the $\tilde{x}$ variables, the number of parametric derivatives of order $q$ and class $i$ at $\tilde{x}_{0}$ is $\tilde{s}_{i}^{(q)}$. If $\mathcal{R}$ is involutive at order $q$ in some variables, we must have $r^{(q+1)}=\sum i \tilde{s}_{i}^{(q)}$. By our results from Chapter 2 this involutivity test for the symbol module will be satisfied at some finite order.

\subsection{A general completion algorithm}

Coupled with the involutivity test from Section 3.1. Theorem 3.0.10 suggests an algorithm for completing a differential equation $\mathcal{R}_{q}$ locally to its involutive form. For simplicity we assume that $\mathcal{R}_{q}$ is determined only by equations of order exactly $q$ and that no integrability conditions of order $<q$ arise during the completion algorithm, referring to Remark 3.0 .3 for the more general case. As always we assume $\mathcal{R}_{q}$ is regular. 


\section{Algorithm 3.2.1.}

(a) First check if $\mathcal{R}_{q, 1}^{(1)}=\mathcal{R}_{q}$ by prolonging all the defining equations. If not, then we replace $\mathcal{R}_{q}$ by $\mathcal{R}_{q, 1}^{(1)}$ and start over. (Recall Remark 3.0.3).

(b) When we stop getting integrability conditions in (a) compute all $q^{\text {th }}$ order reduced Cartan characters (at all points in a neighborhood of interest) using the routine from Section 3.1. If they are not constant we repeat step (a), this time with $\mathcal{R}_{q+1}:=\mathcal{R}_{q, 1}$ instead of $\mathcal{R}_{q}$.

(c) Once we have locally constant reduced Cartan characters in step (b), for some equation $\mathcal{R}_{p}$, we count the number of parametric derivatives of order $p+1$ in $\mathcal{R}_{p, 1}, r^{(p+1)}$, and check whether $r^{(p+1)}=\sum i \tilde{s}_{i}^{(p)}$. If successful, we know that there exist coordinates in which $\mathcal{R}_{p}$ is involutive in the open neighborhood from step (b). If this fails, we go back to (a) with $\mathcal{R}_{p}$ replaced by $\mathcal{R}_{p, 1}$.

Remark 3.2.2. After a few loops through steps (a) and (b) in the above algorithm we may find ourselves working with a $v^{\text {th }}$ order system $\mathcal{R}_{v}$, for $v>q$. If $\mathcal{R}_{v, 1}^{(1)}$ provides a new equation of order $p<v$, we must adjoin that equation to $\mathcal{R}_{p}$, found previously, and repeat step (a). For sake of clarity we suppressed this point above. Notice that this means that, when everything is unwrapped, the equations $\mathcal{R}_{p}$ in step (c) are actually of the form $\mathcal{R}_{q, t}^{(s)}$ for some $s, t$. Again, for clarity, we bypassed keeping track of the $s$ 's and $t$ 's in the above.

Every integrability condition found during Algorithm 3.2.1 enlarges the symbol module of the equation and so, by the Hilbert basis theorem and regularity, we will eventually stop finding integrability conditions. By regularity we will eventually move past step (b) and Cartan's test in step (c) will succeed eventually by Theorem 2.4.4. By Theorem 3.0.10, the result is, after changing variables into the $\delta$-regular ones, an involutive differential equation and the reduced Cartan characters contain information on the order of freeness of a general solution to $\mathcal{R}$, [46]. For example, if $\mathcal{R}_{q}$ is involutive and contains only equations of order exactly $q$, then $\tilde{s}_{i}^{(q)}$ is the 
number of free functions of $i$ variables that the general solution of $\mathcal{R}_{q}$ will depend on.

For our eventual purpose of developing a hybrid equivalence method by mixing the methods of Cartan, [7, and the equivariant moving frame, [43], we rewrite Algorithm 3.2.1 in an equivalent way using the contact forms on $J^{\infty}(\mathcal{E})$. The next few remarks collect the necessary facts.

Remark 3.2.3. We have seen how to deal with integrability conditions of order less that $q$ in Remark 3.0.3, and how we could revert back to considering a collection of equations all of the same order. When looking for integrability conditions in $\mathcal{R}_{q, 1}^{(1)}$, where $\mathcal{R}_{q}$ is determined only by equations of order exactly $q$, we can proceed as follows. Compute the exterior derivative of all $\Upsilon_{\left.J\right|_{\mathcal{R}_{q}}}^{\alpha},|J|=q-1$, and set the coefficients of the purely horizontal parts $d x^{i} \wedge d x^{j}$ equal to zero. To see this, let $\mathcal{R}_{q}$ be given by equations in solved form:

$$
u_{J, k}^{\alpha}=\Phi_{J, k}^{\alpha}(x, \text { parametric derivatives })
$$

where $|J|=q-1$. (We have written $J, k$ to emphasize that the multi-index has non-zero $k^{\text {th }}$ index.) If both $u_{J, j}^{\alpha}$ and $u_{J, i}^{\alpha}, i \neq j$, are principal derivatives in $\mathcal{R}_{q}$, the coefficient of $d x^{j} \wedge d x^{i}$ in $d \Upsilon_{\left.J\right|_{\mathcal{R}_{q}}}^{\alpha}=d\left(d u_{J}^{\alpha}-u_{J, k}^{\alpha} d x^{k}\right)_{\left.\right|_{\mathcal{R}_{q}}}$ will equal

$$
D_{i}\left(\Phi_{J, j}^{\alpha}\right)-D_{j}\left(\Phi_{J, i}^{\alpha}\right)
$$

So we obtain the cross-derivative. If $u_{J, i}^{\alpha}=\Phi_{J, i}^{\alpha}$ is principal and $u_{J, j}^{\alpha}$ parametric, we obtain the coefficient

$$
D_{j}\left(\Phi_{J, i}^{\alpha}\right)-u_{J, j, i}^{\alpha}
$$

which is just the prolongation of $u_{J, i}^{\alpha}=\Phi_{J, i}^{\alpha}$. We claim that the collection $d \Upsilon_{J}^{\alpha}$ provides all prolongations and cross-derivatives from $\mathcal{R}_{q}$ to $\mathcal{R}_{q, 1}$ that are needed for finding integrability conditions. The only non-trivial point of proving this claim is analyzing what happens to the coefficients of the repeated wedge products $d x^{i} \wedge d x^{i}$, since these vanish when taking $d \Upsilon_{J}^{\alpha}$. Consider again the equation (3.8). 
When we compute $d \Upsilon_{\left.J\right|_{\mathcal{R}}}^{\alpha}$ the term

$$
D_{k} \Phi_{J, k}^{\alpha} d x^{k} \wedge d x^{k}
$$

vanishes. We claim that the prolonged equation $u_{J, k, k}^{\alpha}=D_{k} \Phi_{J, k}^{\alpha}$ is only missing from the collection $\left.d \Upsilon_{K}^{\alpha}\right|_{\mathcal{R}_{q}}$ if $u_{J, k, k}^{\alpha}$ is actually the $(|J|+2)$-fold derivative with respect to $x^{k}$. In this case, it is quite obvious that it will not contribute to any integrability condition since it can not be involved in some non-trivial crossderivative. To prove this claim, assume that the multi-indices $L, i, j$ and $J, k, k$ agree (where we can assume that $j \neq k$, since, otherwise, $L, i=J, k$ and $\Phi_{L, i}^{\alpha}=$ $\left.\Phi_{J, k}^{\alpha}\right)$. Then we can write $J, k=N, j$ for $N=(L-k), i$ and computing $d \Upsilon_{\left.N\right|_{\mathcal{R}_{q}}}^{\alpha}$ gives

$$
\begin{aligned}
d \Upsilon_{\left.N\right|_{\mathcal{R}_{q}}=}^{\alpha} & -d u_{N, t}^{\alpha} \wedge d x^{t} \\
= & -d u_{N, j} \wedge d x^{j}-\sum_{t \neq j} d u_{N, t}^{\alpha} \wedge d x^{t} \\
= & -d \Phi_{J, k}^{\alpha} \wedge d x^{j}-\sum_{t \neq j} d u_{N, t}^{\alpha} \wedge d x^{t} \\
= & -D_{k} \Phi_{J, k}^{\alpha} d x^{k} \wedge d x^{j}-\sum_{t \neq k} D_{t} \Phi_{J, k}^{a} d x^{t} \wedge d x^{j} \\
& -d u_{N, k}^{\alpha} \wedge d x^{k}-\sum_{t \neq j, k} d u_{N, t}^{\alpha} \wedge d x^{t}
\end{aligned}
$$

The coefficient of the non-vanishing $d x^{k} \wedge d x^{j}$ in this last expression is

$$
-D_{k} \Phi_{J, k}^{\alpha}+u_{N, k, j}^{\alpha}=-D_{k} \Phi_{J, k}^{\alpha}+u_{J, k, k}^{\alpha}
$$

So if $J, k, k$ is not the $(|J|+2)$-fold derivative with respect to $x^{k}$ we recover the lost prolongation. This proves our claim.

Remark 3.2.4. Let $\mathcal{R}_{q}$ be determined by equations of order exactly $q$ only and assume that no integrability conditions are found going from $\mathcal{R}_{q}$ to $\mathcal{R}_{q, 1}$. Then, when searching for integrability conditions between $\mathcal{R}_{q, 1}$ and $\mathcal{R}_{q, 2}$ we compute all the $d \Upsilon_{\left.L\right|_{\mathcal{R}_{q, 1}}}^{\alpha},|L|=q$, as in Remark 3.2.3, but we notice that we obtain, for the 
principal derivatives $u_{L}^{\alpha}$ in $\mathcal{R}_{q}$, with $u_{L}^{\alpha}=\Phi_{L}^{\alpha}$, that

$$
\Upsilon_{\left.L\right|_{\mathcal{R}_{q, 1}} ^{\alpha}}^{\alpha}=d\left(\Phi_{L}^{\alpha}\right)-D_{j}\left(\Phi_{L}^{\alpha}\right) d x^{j}=d_{V} \Phi_{L}^{\alpha}
$$

and $d \Upsilon_{L}^{\alpha}$ will be a linear combination of $d \Upsilon_{K}^{\beta}$ for $u_{K}^{\beta}$ parametric in $\mathcal{R}_{q}$. Therefore, there is no need to bother computing $d \Upsilon_{L}^{\alpha}$ for principal derivatives $u_{L}^{\alpha}$.

Remark 3.2.5. Recall equation (3.7), the key ingredient in our algorithm for finding $\delta$-regular coordinates. In order to maximize the number of parametric derivatives of certain classes we successively maximized the rank of collections of the form

$$
\left.\left\{\gamma_{q}\left(a_{j}^{i} D_{i}\right\lrcorner d \Upsilon_{J}^{\alpha}\right)_{\left.\right|_{\mathcal{R}_{q}}}\right\}_{|J|=q-1} .
$$

Note that if $\Upsilon_{J}^{\alpha}$ is a principal derivative, that is, one of the determining equations of $\mathcal{R}$ has the form

$$
u_{J}^{\alpha}=\Phi_{J}^{\alpha}\left(x, \ldots, u_{K}^{\beta}, \ldots\right), \quad u_{K}^{\beta} \text { parametric }
$$

then taking the exterior derivative of both sides gives

$$
\Upsilon_{\left.J\right|_{\mathcal{R}}}^{\alpha}=\frac{\partial \Phi_{J}^{\alpha}}{\partial u_{K}^{\beta}} \Upsilon_{K}^{\beta}+\left(D_{i} \Phi_{J}^{\alpha}-u_{J, i}^{\alpha}\right) d x^{i}
$$

This means that, in (3.9), every $d \Upsilon_{J}^{\alpha}$, for $u_{J}^{\alpha}$ principal, can be written as a linear combination of $d \Upsilon_{K}^{\beta}$ for $u_{K}^{\beta}$ parametric. This means that in maximizing the rank of collections of the form (3.9) there is no need to include principal derivatives. Coupled with Remark 3.2 .4 we have shown that, modulo lower order complications, cf. Remark 3.0.3. principal contact forms $\Upsilon_{J}^{\alpha}$ do not enter our computations in searching for integrability conditions or testing for involution. Since parametric derivatives parametrize the equations $\mathcal{R}_{p}$, this is a reflection of the intrinsic approach available to studying differential equations.

Considering the above remarks, we can rewrite Algorithm 3.2.1 in the following way. 


\section{Algorithm 3.2.6.}

(a) First check if $\mathcal{R}_{q, 1}^{(1)}=\mathcal{R}_{q}$ by computing all $d \Upsilon_{K}^{\alpha}$ for $|K|=q-1$ and restricting to $\mathcal{R}_{q}$. If not, then replace $\mathcal{R}_{q}$ by $\mathcal{R}_{q, 1}^{(1)}$ and start over.

(b) When we stop getting integrability conditions in (a), compute $d \Upsilon_{K}^{\alpha},|K|=$ $q-1$ (where it is sufficient to include only parametric derivatives $u_{K}^{\alpha}$ ). Set up the relevant matrices, and compute the reduced Cartan characters, $\tilde{s}_{i}^{(q)}$ in a neighborhood in $\mathcal{R}_{q}$. If they are non constant we repeat step (a), this time with $\mathcal{R}_{q+1}:=\mathcal{R}_{q, 1}$ instead of $\mathcal{R}_{q}$.

(c) Once we have locally constant reduced Cartan characters in step (b), for some system $\mathcal{R}_{p}$, we count the number of parametric derivatives of order $p+1$ in $\mathcal{R}_{p, 1}, r^{(p+1)}$, and check whether $r^{(p+1)}=\sum i \tilde{s}_{i}^{(p)}$. If successful, we know that there exist coordinates in which $\mathcal{R}_{p}$ is involutive in the open neighborhood from step (b). If this fails, we go back to (a) with $\mathcal{R}_{p}$ replaced by $\mathcal{R}_{p, 1}$. By Remark 3.2.4, when we go back to (a) we need only compute $d \Upsilon_{K}^{\alpha}$ for $|K|=p$ and parametric $u_{K}^{\alpha}$ only.

Remark 3.2.7. Although computing the exterior derivatives of contact forms restricted to differential equations is sufficient for prolongation it is not, in general, practical since it involves many unnecessary computations. However, in studying symmetry problems for Lie pseudo-groups where the full geometric structure of the differential system is of interest, when coupled with the recurrence formula from the method of equivariant moving frames, [42, the above algorithm is very valuable indeed.

In order to make an explicit connection to the original equivalence method in later sections, we mention here the "intrinsic" approach to prolonging differential equations.

Remark 3.2.8. The straightforward way of prolonging a differential equation 
$F\left(x, u^{(q)}\right)=0$ is applying the total derivative operator

$$
D_{i}=\frac{\partial}{\partial x^{i}}+\sum_{|J| \geq 0} u_{J, i}^{\alpha} \frac{\partial}{\partial u_{J}^{\alpha}} .
$$

This essentially amounts to identifying $\mathcal{R}_{q}$ with its preimage $\left(\pi_{q}^{\infty}\right)^{-1}\left(\mathcal{R}_{q}\right) \in J^{\infty}(\mathcal{E})$ and performing the computations on this larger space (indeed, $D_{i}$ is only defined on the infinite bundle $J^{\infty}(\mathcal{E})$ ). A more intrinsic approach to the differential equation $\mathcal{R}_{q}$ is available by looking at its contact co-distribution $\boldsymbol{C}_{q}$ in $T^{*} \mathcal{R}_{q}$. This is the co-distribution generated by the one-forms $\Upsilon_{J}^{\alpha}$, for $|J|<q$, restricted (or pulled-back) to $\mathcal{R}_{q}$. An integral element, $E$, of this co-distribution at the point $\left(x, u^{(q)}\right) \in \mathcal{R}_{q}$ is an $n$-dimensional subspace of $T_{\left(x, u^{(q)}\right)} \mathcal{R}_{q}$ that is transverse to the fibration $\pi_{0}^{q}: \mathcal{R}_{q} \rightarrow \mathbb{R}^{n}$ and annihilates the co-distribution,

$$
\left.\left(\Upsilon_{\left.J\right|_{\mathcal{R}_{q}}}^{\alpha}\right)\right|_{E}=0, \quad|J|<q
$$

We introduce the truncated total derivatives on $J^{q}(\mathcal{E})$,

$$
\widetilde{D}_{i}^{q}=\frac{\partial}{\partial x^{i}}+\sum_{|J|<q} u_{J, i}^{\alpha} \frac{\partial}{\partial u_{J}^{\alpha}}
$$

It is easily seen that any integral element $E$ of $\boldsymbol{C}_{q}$ has a basis $\left\{\mathbf{V}_{1}, \ldots, \mathbf{V}_{n}\right\}$ of the form

$$
\mathbf{V}_{i}=\widetilde{D}_{i}^{q}+\sum_{|J|=q} z_{J ; i}^{\alpha} \frac{\partial}{\partial u_{J}^{\alpha}},
$$

for coefficients $z_{J ; i}^{\alpha}$ that must satisfy certain linear equations. These equations arise from the fact that $E$ must not only annihilate $\Upsilon_{J_{\mathcal{R}_{q}}}^{\alpha}$ but also its exterior derivative. For example, when confined to $\mathcal{R}_{q}$, we have

$$
d \Upsilon_{J}^{\alpha}=-d u_{J, i}^{\alpha} \wedge d x^{i}, \quad|J|<q
$$


and the fact that $E=\operatorname{Span}\left(\mathbf{V}_{1}, \ldots, \mathbf{V}_{n}\right)$ is an integral element means that

$$
-\left.d u_{J, i}^{\alpha} \wedge d x^{i}\right|_{\mathcal{R}_{q}}\left\langle\mathbf{V}_{k}, \mathbf{V}_{l}\right\rangle=0
$$

When $u_{J, i}^{\alpha}$ is a principal derivative in $\mathcal{R}_{q}$, i.e. if we write one of the defining equations of $\mathcal{R}_{q}$ in a "solved form"

$$
u_{J, i}^{\alpha}=\Phi\left(x, \ldots, u_{K}^{\beta}, \ldots\right), \quad u_{K}^{\beta} \text { parametric }
$$

we have, on $\mathcal{R}_{q}$,

$$
d u_{J, i}^{\alpha}=\widetilde{D}_{j}^{q} \Phi d x^{j}+\sum_{|K|=q} \frac{\partial \Phi}{\partial u_{K}^{\beta}} d u_{K}^{\beta}+\text { contact forms. }
$$

Evaluating $d u_{J, i}^{\alpha}\left\langle\mathbf{V}_{k}\right\rangle$ then gives

$$
\widetilde{D}_{k}^{q} \Phi+z_{K ; k}^{\beta} \frac{\partial \Phi}{\partial u_{K}^{\beta}}
$$

We can see that identifying $z_{K ; k}^{\beta}$ with the jet-coordinate $u_{K, k}^{\beta}$, equation 3.11 becomes the prolongation of $u_{J, i}^{\alpha}=\Phi$. In general, the equations (3.10) will amount to the same exact equations as if we had straightforwardly prolonged $F\left(x, u^{(q)}\right)=0$ using the non-restricted total derivatives $D_{i}$. (See Proposition 2.3.19 in [46].) In particular, the number of parametric derivatives of order $q+1$ in the equations for $\mathcal{R}_{q, 1}$ is the same as the number of free variables $z_{J ; i}^{\alpha}$ in the equations $(3.10)$.

The projections of all "integral vectors" $\mathbf{V}_{i}=\widetilde{D}_{i}^{q}+\sum_{|J|=q} z_{J ; i ;}^{\alpha} \frac{\partial}{\partial u_{J}^{\alpha}}$, that annihilate $\boldsymbol{C}_{q}$, onto the top order part,

$$
\sum_{|J|=q} z_{J ; i}^{\alpha} \frac{\partial}{\partial u_{J}^{\alpha}}
$$

form an algebraic object, intrinsically associated with the differential equation, called its symbol co-module. Its Spencer cohomology was used by Spencer and 
Kodaira et al. for a geometric theory of differential equations, [32]. The Spencer cohomology approach to differential equations is equivalent to the Pommaret bases approach we have adopted, although it must be admitted that the former approach is much more difficult to apply to specific examples. See [46] for the full story.

\subsection{Cartan normal form and regularity}

In this section we discuss the consequences of our regularity condition in Definition 3.0.6. In particular, we shall prove that it is equivalent to being able to write it locally, possibly after some prolongation/projection, in Cartan normal form. This is not so trivial and resolves many of the regularity issues inherent in Cartan's equivalence method as well as the groupoid approach to Lie pseudo-groups (see Appendix). Since the Cartan normal form is necessary for the application of the Cartan-Kähler theorem this regularity condition is very natural indeed.

Let $\mathcal{R}_{q}$ be regular. Algorithm 3.2 .6 completes $\mathcal{R}_{q}$ to a system $\mathcal{R}_{q, t}^{(s)}$ that is involutive. For notational convenience, we assume that $\mathcal{R}_{q}$ itself is involutive, i.e. that $s=t=0$. A great simplifying trick in the formal theory of differential equations is rewriting $\mathcal{R}_{q}$ as a system of of order one. This is achieved by setting the jet coordinates $u_{J}^{\alpha},|J| \leq q-1$, as the new dependent variables and setting up the following system of equations among them. (We shall write $u_{J ; i}^{\alpha}$ for $\frac{\partial u_{J}^{\alpha}}{\partial x^{i}}$.)

$$
\begin{aligned}
u_{J ; i}^{\alpha} & =u_{J, i}^{\alpha}, \quad \text { for }|J|<q-1, \\
u_{J-i+j ; i}^{\alpha} & =u_{J ; j}^{\alpha}, \quad \text { for }|J|=q-1 \text { where } J_{i} \neq 0 .
\end{aligned}
$$

If $\Phi\left(x, u^{(q)}\right)=0$ is one of the equations for $\mathcal{R}_{q}$ then we replace the $u_{J}^{\alpha},|J|=q$, by $u_{J-k ; k}^{\alpha}$ where $k$ is the class of $J$. This specific way of rewriting $\mathcal{R}_{q}$ as an equivalent first order system preserves the Cartan characters of the system, see [46. Proposition A.3.1]. The first order system also has the same reduced Cartan characters as its progenitor so the regularity of Definition 3.0.6 is also preserved. This all means that we can assume that $\mathcal{R}_{q}=\widetilde{\mathcal{R}}_{1}$ is first order where the analysis 
is much easier. Notice that any equation of order $<q$ in $\mathcal{R}_{q}$ becomes a zero order equation in the new variables. Regularity stipulates, among other things, that $\pi_{q-1}^{q}\left(\mathcal{R}_{q}\right)$ be a submanifold in $J^{q-1}(\mathcal{E})$ and so we can, locally, write these zero order equations in solved form

$$
\begin{aligned}
u^{\sigma^{1}} & =\Phi_{0}^{1}\left(x, u^{\tau^{1}}, \ldots, u^{\tau^{l}}\right) \\
& \vdots \\
u^{\sigma^{k}} & =\Phi_{0}^{k}\left(x, u^{\tau^{1}}, \ldots, u^{\tau^{l}}\right),
\end{aligned}
$$

where $n+l$ is the dimension of $\pi_{q-1}^{q}\left(\mathcal{R}_{q}\right)$ as a manifold while $n+l+k$ is the dimension of $J^{q-1}(\mathcal{E})$. The fact that $\mathcal{R}_{q, 1}^{(1)}=\mathcal{R}_{q}$ means that any prolongation of these zero order equations is an algebraic consequence of equations already in $\widetilde{\mathcal{R}}_{1}$. It is then routine to show that any solution to the top order equations only, that satisfy the zero order equations at a single point, is a solution to the entire system (see Remark 3.0.3 for this a similar argument).

Next we show that the constancy of the reduced Cartan characters of $\widetilde{\mathcal{R}}_{1}$, and its involution, means that we can, in a neighborhood of any point of interest in $\widetilde{\mathcal{R}}_{1}$, choose the same set of parametric derivatives with respect to the degree reverse lexicographic order in the $\delta$-regular coordinates. Although this is rather easy to prove it is an important point. For a formally integrable equation, involution of its symbol at a single point does not guarantee local solvability via the CartanKäher theorem since the structure of the symbol can vary from point to point preventing a Cartan normal form presentation of the system. A simple example is the following modification of Example 3.0 .7 .

Example 3.3.1. Dropping all the second order equation from Example 3.0.7, we consider the first order, non-regular, formally integrable, differential system $\mathcal{R}_{1}$ for maps

$$
(x, y, u) \mapsto(X(x, y, u), Y(x, y, u), U(x, y, u))
$$


for real valued functions $X, Y$ and $U$, determined by the equations

$$
X=x, \quad Y=y, \quad U=u+x U_{x}+y U_{y} .
$$

The symbol of $\mathcal{R}_{1}$, and all its prolongations, changes rather drastically close to $x=0$ and $y=0$ and on their intersection, the $u$-axis of the base manifold $\mathbb{R}^{3}$, it assumes a more degenerate form still as the determining equations decrease in order there. At all points in fibers above the $u$-axis the symbol module is all of $\mathbb{R}\left[\xi^{1}, \xi^{2}, \xi^{3}\right] \otimes \mathbb{R}^{3}$ and hence trivially involutive. However, the lack of regularity at these points prevents an application of the Cartan-Kähler theorem at these points (at all orders). Away from the $u$-axis the system is regular and hence locally solvable (by the results below).

We have seen that the search for $\delta$-regular coordinates (at a single point $j^{1} u_{\left.\right|_{x}} \in$ $\left.\widetilde{\mathcal{R}_{1}}\right)$ can be facilitated by successively maximizing the ranks of $\mathcal{C}\left(v_{1}\right) \cup \cdots \cup \mathcal{C}\left(v_{k}\right)$, where

$$
\left.\mathcal{C}\left(v_{i}\right)=\left\{\gamma_{q}\left(a_{i}^{j} D_{j}\right\lrcorner d \Upsilon^{\alpha}{ }_{\left.\right|_{\mathcal{R}_{1}}}\right)\right\}_{0}, \quad v_{i}=\left(a_{i}^{1}, \ldots, a_{i}^{n}\right) \in \mathbb{R}^{n} .
$$

Consider the collection of one forms

$$
\left.\mathcal{C}\left(v_{1}\right)=\left\{\gamma_{1}\left(a_{1}^{j} D_{j}\right\lrcorner d \Upsilon^{\alpha}{ }_{{ }_{\mathcal{R}_{1}}}\right)\right\}_{0}=\left\{\gamma_{1}\left(a_{1}^{j} \Upsilon_{j}^{\alpha} \widetilde{\mathcal{R}}_{1}\right)\right\}_{0} .
$$

Now imagine we maximize the rank of this system not merely over all $v_{1} \in \mathbb{R}^{n}$ but also all points $j^{1} u_{\left.\right|_{x}} \in \widetilde{\mathcal{R}}_{1}$. Of course, since all points in $\widetilde{\mathcal{R}_{1}}$ have the same reduced Cartan characters this maximum is attained at all points, and in particular at $j^{1} u_{\left.\right|_{x}}$. Since maximizing the rank of $\mathcal{C}\left(v_{1}\right)$ boils down to maximizing the rank of a certain matrix whose entries are functions of $v_{1}$ and $\left(x, u^{(1)}\right)$ the same $\tilde{s}_{1}^{(1)}$ rows can be chosen as a basis for the row space at all points in an open neighborhood $U$ of $j^{1} u_{\left.\right|_{x}}$ and for the same vector $\tilde{v}_{1} \in \mathbb{R}^{n}$. This is simply because a fixed set of rows generate the row space on a set determined by the non-vanishing of certain minors and is therefore open in $\mathbb{R}^{n} \times \widetilde{\mathcal{R}}_{1}$. In fact, the set on which a certain collection of rows generate the row space of $\mathcal{C}\left(v_{1}\right)$ is either empty or dense in $\mathbb{R}^{n} \times \widetilde{\mathcal{R}}_{1}$. 
The rows that generate the row space correspond to elements of $\mathcal{C}\left(\tilde{v}_{1}\right)$ that are a basis for the $\mathbb{R}$ vector space that $\mathcal{C}\left(\tilde{v}_{1}\right)$ generates in $\Gamma(1)$ (cf. Section 3.1). Let the subcollection

$$
\gamma_{1}\left(a_{1}^{j} \Upsilon_{j}^{\alpha^{1} \mid \widetilde{\mathcal{R}}_{1}}\right), \ldots, \gamma_{1}\left(a_{n}^{j} \Upsilon_{j}^{\alpha^{\tilde{s}^{(1)}} \mid \widetilde{\mathcal{R}}_{1}}\right), \quad \tilde{v}_{1}=\left(a_{1}^{1}, \ldots, a_{1}^{n}\right)
$$

be such a basis. When we write our system in new variables with $\bar{x}^{1}=\tilde{v}_{1} \cdot x$ and collect those equations that only involve $u_{\bar{x}^{1}}^{\alpha}=\frac{\partial u^{\alpha}}{\partial \bar{x}^{1}}$ we can see that according to the above $u_{\bar{x}^{1}}^{\alpha^{1}}, \ldots, u_{\bar{x}^{1}}^{\alpha^{\tilde{s}^{(1)}}}$ can be chosen to be parametric in the open set $U$. In general, denote the number, dual to $\tilde{s}_{i}^{(1)}$, of principal derivatives of class $i$ by $\tilde{\beta}_{i}^{(1)}$. Continuing the above argument, the maximum rank of $\mathcal{C}\left(v_{1}\right) \cup \mathcal{C}\left(v_{2}\right)$ over all pairs $v_{1}, v_{2}$ and all points in $U$ is attained at $\tilde{v}_{1}, \tilde{v}_{2}, j^{1} u_{\left.\right|_{x}}$, for some $\tilde{v}_{2} \in \mathbb{R}^{n}$, and by the same arguments as above, we can choose the same $\tilde{s}_{2}^{(1)}$ derivatives with respect to $\bar{x}^{2}=\tilde{v}_{2} \cdot x$ to be parametric in a neighborhood of $j^{1} u_{\left.\right|_{x}}$ in $\widetilde{U}$. Continuing this, and possibly replacing $U$ by a smaller open set, and possibly relabeling the $u^{\alpha}$, involutivity and formal integrability mean that we can write the top order equations in $\widetilde{\mathcal{R}}_{1}$ in the $\delta$-regular coordinates in Cartan normal form,

$$
\begin{array}{cl}
u_{\bar{x}^{1}}^{\alpha}=\Phi_{1}^{\alpha}(x, u, \text { parametric derivatives of class } \leq 1), & 1 \leq \alpha \leq \tilde{\beta}_{1}^{(1)} \\
u_{\bar{x}^{2}}^{\alpha}=\Phi_{2}^{\alpha}(x, u, \text { parametric derivatives of class } \leq 2), & 1 \leq \alpha \leq \tilde{\beta}_{2}^{(1)} \\
\vdots & \\
u_{\bar{x}^{n}}^{\alpha}=\Phi_{n}^{\alpha}(x, u, \text { parametric derivatives of class } \leq n), \quad 1 \leq \alpha \leq \tilde{\beta}_{n}^{(1)} .
\end{array}
$$

Notice that involutivity implies $\tilde{\beta}_{1}^{(1)} \leq \tilde{\beta}_{2}^{(1)} \leq \cdots \leq \tilde{\beta}_{n}^{(1)}$. The Cartan-Kähler theorem now applies to this system (and its lower order part (3.13)).

Remark 3.3.2. Any general discussion of the Cartan normal form will unfortunately and unavoidably be somewhat notationally cluttered. The key lesson of the above discussion is that our regularity concept guarantees that around each 
point in an involutive system $\mathcal{R}_{q}$ we can choose the same set of parametric monomials of each class in an open neighborhood. This enables the fundamental Cartan normal form and hence local solvability almost everywhere. As mentioned above our regularity concept frees us from some historically tricky issues in Cartan's equivalence method, specifically relating to the concept of equivalence problems of constant type, and allows for a more general formulation of Cartan's method.

Remark 3.3.3. Notice that the arguments above imply that differential equations satisfying our blanket assumption from Remark 3.0.5 are regular on a dense open subset. Similarly, in the appendix, we present a proof that the determining equations of Lie pseudo-groups are always regular in a dense, open and invariant set.

Remark 3.3.4. Obviously, any equation in Cartan normal form (3.14), is regular. Conversely, Algorithm 3.2.6 completes any regular system to an involutive one that, by the above discussion, can be written in Cartan normal form. So for a system $\mathcal{R}_{q}$ the following are equivalent.

1. There exist $s \leq t$ such that $\mathcal{R}_{q, t}^{(s)}$ can be written in Cartan normal form.

2. $\mathcal{R}_{q}$ is regular.

The above shows that our notion of regularity is necessary and sufficient for any study of local solvability via the Cartan-Kähler theorem. 


\section{Chapter 4}

\section{Pseudo-groups}

This chapter gives a rapid overview of the groupoid approach to Lie pseudogroups, their structure equations and the all-important recurrence formula for lifted invariants, developed in the series of papers [42, 43, 44]. We only present a proof of the recurrence formula in the special case of horizontal actions, but the general case is identical, see [43]. We remind the reader that all differential equations are assumed regular in the sense of Definition 3.0.6.

\subsection{Basic objects}

Consider the jet bundle $J^{\infty}(\mathcal{X} \times \mathcal{X})$ for sections of the trivial bundle $\mathcal{X} \times \mathcal{X} \stackrel{\sigma}{\rightarrow} \mathcal{X}$ where $\mathcal{X}$ is an $n$-dimensional manifold. Let $\mathcal{D}(\mathcal{X})$ denote the collection of all local diffeomorphisms of $\mathcal{X}$ and let $\mathcal{D}_{\infty}(\mathcal{X}) \subset J^{\infty}(\mathcal{X} \times \mathcal{X} \rightarrow \mathcal{X})$ be the subbundle of all infinite jets of these. We shall drop the mention of $\mathcal{X}$ when it is clear what the base manifold is and simply write $\mathcal{D}$ and $\mathcal{D}_{\infty}$ instead of $\mathcal{D}(\mathcal{X})$ and $\mathcal{D}_{\infty}(\mathcal{X})$. Similarly, we denote by $\mathcal{D}_{p}(\mathcal{X})$ the set of $p$-jets of transformations from $\mathcal{D}(\mathcal{X})$. For local coordinates $x$ on $\mathcal{X}$ we have the induced jet coordinates $\left(x, X, \ldots, X_{K}^{i}, \ldots\right)$ on $\mathcal{D}_{\infty}$ (and by truncation on $\mathcal{D}_{p}$ ). That is, for a local diffeomorphism $\varphi$, we have 
$j^{\infty} \phi_{\left.\right|_{x}}=\left(x, X, \ldots, X_{K}^{i}, \ldots\right)$, where

$$
X_{K}^{i}=\frac{\partial^{|K|} \varphi}{\partial x^{K}}(x), \quad K \in \mathbb{N}_{0}^{n} .
$$

The collection $\mathcal{D}$ forms a pseudo-group, since if $\varphi \in \mathcal{D}$ then $\varphi^{-1} \in \mathcal{D}$ and the composition of two local diffeomorphisms is again a diffeomorphism whenever the composition can be defined. As emphasized by Ehresmann, [11], each set $\mathcal{D}_{p} \subset J^{p}(\mathcal{X} \times \mathcal{X})$ carries a groupoid structure; we define the source and target of a $p$-jet $j^{p} \varphi_{\left.\right|_{x}}=\left(x, X, \ldots, X_{L}^{i}, \ldots\right) \in \mathcal{D}_{p}$ as

$$
\sigma\left(j^{p} \varphi_{\left.\right|_{x}}\right)=x \quad \text { and } \quad \tau\left(j^{p} \varphi_{\left.\right|_{x}}\right)=X
$$

respectively. The groupoid multiplication of $j^{p} \varphi_{\left.\right|_{x}}$ and $j^{p} \psi_{\left.\right|_{X}}$, where $\tau\left(j^{p} \varphi_{\left.\right|_{x}}\right)=$ $\sigma\left(j^{p} \psi_{\left.\right|_{X}}\right)$, is defined as

$$
\left.j^{p}(g \circ f)\right|_{x},
$$

where $f$ and $g$ are functions in $\mathcal{D}$ having the $p$-jets $j^{p} f_{\left.\right|_{x}}=j^{p} \varphi_{\left.\right|_{x}}$ and $j^{p} g_{\left.\right|_{X}}=$ $j^{p} \psi_{\left.\right|_{X}}$. This definition does not depend on the choice of $f$ and $g$ as can be seen from the chain rule. We write the groupoid operation as $j^{p} \psi_{\left.\right|_{X}} \cdot j^{p} \varphi_{\left.\right|_{x}}$. The source and target maps provide each $\mathcal{D}_{p}$ with a double fibration,

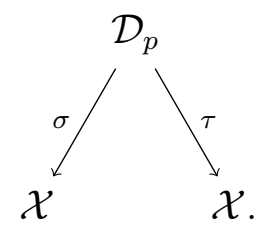

Definition 4.1.1. A Lie pseudo-group, $\mathcal{G}$, of local transformations of $\mathcal{X}$ is a subpseudo-group of $\mathcal{D}$ that is determined by a set of formally integrable differential equations called the defining equations that are regular in the sense of Definition 3.0 .6 .

As above, we denote the collection of transformations making up the pseudogroup by $\mathcal{G}$ while subscripts will indicate the set of groupoid elements, e.g. $\mathcal{G}_{\infty}$ 
is the set of infinite jets of transformations from $\mathcal{G}$. Each $\mathcal{G}_{p}, 0 \leq p \leq \infty$, is a sub-groupoid of $\mathcal{D}_{p}$. Let $\mathcal{G}$ be a Lie pseudo-group determined by the formally integrable equations

$$
F\left(x, X^{(q)}\right)=0
$$

where $X^{(q)}$ denotes all jets up to order $q$. Let $\Phi_{\varepsilon}$ be a one parameter family of diffeomorphisms from $\mathcal{G}$. The flow $\varepsilon \mapsto \Phi_{\varepsilon}(x)$ through points $x \in \mathcal{X}$ generates a vector field

$$
\mathbf{v}(x)=\zeta^{i}(x) \frac{\partial}{\partial x^{i}}
$$

in the Lie algebroid $\mathcal{A}$ of $\mathcal{G}$ of local vector fields on $\mathcal{X}$. The components of $\mathbf{v}$ satisfy the linearization of 4.1 at the identity section $\mathbb{1}$ :

$$
L\left(x, \zeta^{(q)}\right)=\left.\frac{\partial F\left(x, X^{(q)}\right)}{\partial X_{K}^{i}}\right|_{\mathbb{1}} \zeta_{K}^{i}=0
$$

We shall prove, in the appendix, that, since we have assumed the defining equations are regular, (4.3) are locally solvable whenever (4.1) are.

Now assume that the action of $\varphi \in \mathcal{G}$ on $\mathcal{X}$, given by $x \mapsto \varphi(x)$, is extended to a trivial bundle $\mathcal{E}=\mathcal{X} \times \mathcal{U} \stackrel{\pi}{\rightarrow} \mathcal{X}$ where $\mathcal{U} \subset \mathbb{R}^{m}$ is an open set and the action on $\mathcal{U}$ depends only on jets of order 1 (it is easy to extend all results to a general integer $N>1$ but we restrict to 1 for simplicity). This means that

$$
j^{1} \varphi_{\left.\right|_{x}} \cdot(x, u)=\left(\varphi(x), U\left(x, u, j^{1} \varphi_{\left.\right|_{x}}\right)\right)
$$

where $U$ is a function of $x, u$ and $j^{1} \varphi_{\mid}$. Note that we could encode this by a Lie pseudo-group of transformations, $\mathcal{H}$, on $\mathcal{E}$, with defining equations given by those for $\mathcal{G}$ plus the first order equations

$$
U=U\left(x, u, j^{1} \varphi_{\left.\right|_{x}}\right)
$$

where $\varphi$ is the transformation of the first $n$ variables $x \in \mathcal{X}$. The Lie pseudo-group $\mathcal{H}$ is a one-to-one prolongation of $\mathcal{G}$ in the language of [47]. We call extended group 
actions of the form (4.4) horizontal group actions.

By prolongation, $\mathcal{G}$ acts on each $J^{k}(\mathcal{E}) \stackrel{\pi^{k}}{\rightarrow} \mathcal{X}$, where the action on $J^{k}(\mathcal{E})$ depends only on pseudo-group jets of order $1+k$. We refer to the jet bundle $J^{\infty}(\mathcal{E})$ as the submanifold jet bundle, the submanifolds in question being the sections of $\mathcal{E}$ which are central objects in what is to come. We denote the submanifold jet coordinates by $u_{J}^{\alpha}$, where $1 \leq \alpha \leq m$ and $J \in \mathbb{N}_{0}^{n}$ and write $z_{\left.\right|_{x}}^{(\infty)}=\left(x, u, \ldots, u_{J}^{\alpha}, \ldots\right)$ for infinite jets.

We also form the pull-back bundle $\left(\pi^{\infty}\right)^{*} \mathcal{G}_{\infty} \rightarrow J^{\infty}(\mathcal{E})$, with induced coordinates $\left(x, u, \ldots, u_{J}^{\alpha}, \ldots, X, \ldots, X_{K}^{i}, \ldots\right)=\left(z_{\left.\right|_{x}}^{(\infty)}, j^{\infty} \phi_{\left.\right|_{x}}\right)$ and write $\widetilde{\mathcal{G}}_{\infty}$ for the pull-back $\left(\pi^{\infty}\right)^{*} \mathcal{G}_{\infty}$. We extend the source and target maps to $\widetilde{\mathcal{G}}$ by

$$
\tilde{\sigma}\left(\left.z\right|_{x} ^{(\infty)}, j^{\infty} \phi_{\left.\right|_{x}}\right)=\left.z\right|_{x} ^{(\infty)}, \quad \tilde{\tau}\left(\left.z\right|_{x} ^{(\infty)}, j^{\infty} \phi_{\left.\right|_{x}}\right)=\left.j^{\infty} \phi_{\left.\right|_{x}} \cdot z\right|_{x} ^{(\infty)}
$$

providing $\widetilde{\mathcal{G}}_{\infty}$ with a double fibration,

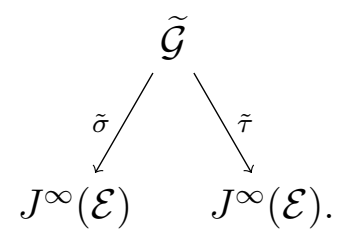

We shall denote the target variables on $J^{\infty}(\mathcal{E})$ by capital letters, that is

$$
Z^{(\infty)}=j^{\infty} \phi_{\left.\right|_{x}} \cdot z_{\left.\right|_{x}}^{(\infty)}=j^{\infty} \phi_{\left.\right|_{x}} \cdot\left(x, u, \ldots, u_{J}^{\alpha}, \ldots\right)=\left(X, U, \ldots, U_{J}^{\alpha}, \ldots\right),
$$

and when we explicitly write out the partial derivatives in $U_{J}^{\alpha}$, they shall be capitalized also, for example

$$
j^{3} \varphi_{\left.\right|_{x}} \cdot\left(x, u, u_{x}, u_{x x}\right)=\left(X, U, U_{X}, U_{X X}\right)
$$

and so on.

Remark 4.1.2. The formula for a lifted invariant depends on the jets, $X, \ldots, X_{K}^{i}, \ldots$, 
of pseudo-group elements $\varphi \in \mathcal{G}$. Since these jets must satisfy the defining equations of $\mathcal{G}$ we may replace each principal derivative by parametric ones in these formulas. After a choice of principal and parametric derivatives, the latter are thought of, and referred to as group parameters.

We give a few examples of horizontal actions. Most of these will be studied in detail in later sections.

Example 4.1.3. Let $\mathcal{G}$ be the pseudo-group of point transformations on $J^{1}(\mathbb{R} \times$ $\mathbb{R} \rightarrow \mathbb{R})$ with coordinates $\left(x, u, u_{x}\right)=(x, u, p)$. This means $X$ and $U$ are functions of $(x, u)$ only and $P$ is given by

$$
P=\frac{U_{x}+p U_{u}}{X_{x}+p X_{u}}
$$

Say we are interested in the effect of these point transformations on second order ODE $u_{x x}=q=f(x, u, p)$. Then, under $(x, u, p) \mapsto(X, U, P), q$ tranforms according to

$$
q \mapsto Q=\frac{P_{x}+p P_{u}+q P_{p}}{X_{x}+p X_{u}} .
$$

We are therefore in the above set-up with $\mathcal{X}$ parametrized by $(x, u, p)$ and $\mathcal{U}$ parametrized by $q$. This example generalizes, of course, to any order above two also.

Example 4.1.4. In studying the local invariants of Riemannian metrics on, say, two dimensional manifolds, we are interested in the effect of a smooth change of coordinates on the components of the tensor and their jets. Let the metric in local coordinates $x \in U \subset \mathbb{R}^{2}$ be $g=g_{i j} d x^{i} d x^{j}$. A change of variables is an invertible map $\varphi: U \rightarrow V \subset \mathbb{R}^{2}$. This will transform $g$ according to

$$
\left(\varphi^{-1}\right)^{*}\left(g_{i j} d x^{i} d x^{j}\right)=g_{i j} d\left(\varphi^{-1}\right)^{i} d\left(\varphi^{-1}\right)^{j}
$$

and so the transformation of the components $g_{i j}$ will depend on the 1-jets of $\varphi$. We therefore have a horizontal action where $\mathcal{G}$ is the pseudo-group of all local 
diffeomorphisms of $\mathbb{R}^{2}$ and $\mathcal{U}$ is the space of symmetric, non-degenerate metrics, parametrized by $g_{i j}, 1 \leq i \leq j \leq 2$, with $g_{i j}=g_{j i}$.

More generally, the components, $u$, of any tensor on a manifold will transform, under a change of variables on the base manifold, $x \mapsto \varphi(x)$, as a function of $x, u$ and $j^{1} \varphi_{\left.\right|_{x}}$.

Example 4.1.5. Consider the Lie pseudo-group of transformations

$$
X=f(x), \quad Y=f_{x}(x) y+g(x),
$$

extended to an additional real variable $u$ by

$$
U=u+\frac{f_{x x}(x) y+g_{x}(x)}{f_{x}(x)}
$$

where $f: \mathbb{R} \rightarrow \mathbb{R}$ is a local, invertible, real-analytic map and $g: \mathbb{R} \rightarrow \mathbb{R}$ is an arbitrary real-analytic map. This pseudo-group has the structure described above: The transformation of the $x$ and $y$ coordinates form a Lie pseudo-group $\mathcal{G}$ with defining equations $X_{y}=Y_{y}-X_{x}=0$ and $\mathcal{G}$ acts on the $u$ coordinate by $u \mapsto U=u+Y_{x} / X_{x}$. This pseudo-group is actually of historical interest rather than geometric as it is related to one of Medolaghi's pseudo-groups, [34].

Example 4.1.6. Let $\int L(x, u, p) d x$ be a first order Lagrangian in one variable. A point transformation $(x, u, p) \mapsto(X, U, P)$ transforms $\int L(x, u, p) d x$ according to

$$
\int L(X, U, P) d X=\int L(X, U, P) \cdot\left(X_{x}+p X_{u}\right) d x
$$

We can set up the problem of equivalence of Lagrangians by extending the standard pseudo-group of contact transformations on $J^{1}\left(\mathbb{R}^{2} \rightarrow \mathbb{R}\right)$ to act on a space parametrized by a real variable $L$ via

$$
L \mapsto \frac{L}{X_{x}+p X_{u}}
$$

More generally, the divergence equivalence of Lagrangians can be cast in this 
framework. In this case we require that $L_{p p}$ is preserved, as opposed to $L$. See Example 5.4 .10 for more.

Example 4.1.7. Consider a linear second order differential operator on $\mathbb{R}$,

$$
\mathscr{D}=f D^{2}+g D+h
$$

where $f, g, h: \mathbb{R} \rightarrow \mathbb{R}$ are real-analytic, and $f \neq 0$. When we apply $\mathscr{D}$ to a real-analytic function $u: \mathbb{R} \rightarrow \mathbb{R}$ we obtain the function

$$
f u^{\prime \prime}+g u^{\prime}+h u
$$

Now consider the pseudo-group, $\mathcal{G}$, of transformations of the $(x, u)$ of the form

$$
(x, u) \mapsto(\varphi(x), u \cdot \psi(x))=(X, U),
$$

where $\varphi, \psi: \mathbb{R} \rightarrow \mathbb{R}$ are real-analytic. Restricting to the set $\mathcal{X}=\{(x, u) \in$ $\left.\mathbb{R}^{2} \mid u>0\right\}$ and to $\psi>0$ this is a Lie pseudo-group of transformations of $\mathcal{X}$ that has defining equations

$$
X_{y}=0, \quad U_{u u}=0, \quad U_{u} u=U
$$

The elements of $\mathcal{G}$ preserve the space of linear operators and a transformation from $\mathcal{G}$ maps 4.5 to

$$
\overline{\mathscr{D}}=F \bar{D}^{2}+G \bar{D}+H
$$

where $\bar{D}$ is the derivative with respect to the transformed independent variables 
$X$ and the lifted coefficients $F, G, H$ have explicit formulas

$$
\begin{aligned}
& F=f \frac{X_{x}^{2}}{U_{u}}, \\
& G=-f \frac{2 U_{x} X_{x}-X_{x x} U_{u} u}{u U_{u}^{2}}+g \frac{X_{x}}{U_{u}}, \\
& H=-f \frac{U_{x x} U_{u} u-2 U_{x}^{2}}{u^{2} U_{u}^{3}}-g \frac{U_{x}}{u U_{u}^{2}}+\frac{h}{U_{u}} .
\end{aligned}
$$

Notice that since $\frac{U_{x}}{u}$ and $U_{u}$ are independent of $u$, these lifted invariants are also independent of $u$ (as they should be be). Each operator $\mathscr{D}$ defines a section,

$$
(x, u) \mapsto(x, u, f(x), g(x), h(x)),
$$

in the trivial bundle $\mathcal{X} \times \mathbb{R}^{3} \rightarrow \mathcal{X}$ and two operators are equivalent if their respective sections are congruent under the extended action of $\mathcal{G}$ given by 4.6 .

\subsection{Lifted invariants}

A local transformation $\psi \in \mathcal{G}$ acts from the right on the set of jets $j^{\infty} \phi_{\left.\right|_{x}} \in \mathcal{G}_{\infty}$ with $x \in \operatorname{dom} \psi$ by

$$
R_{\psi} \cdot j^{\infty} \phi_{\left.\right|_{x}}=\left.j^{\infty} \varphi_{\left.\right|_{x}} \cdot j^{\infty} \psi^{-1}\right|_{\psi(x)},
$$

and from the left on the set of jets $j^{\infty} \phi_{\left.\right|_{x}} \in \mathcal{G}_{\infty}$ with $\tau\left(j^{\infty} \varphi_{\left.\right|_{x}}\right) \in \operatorname{dom} \psi$ by

$$
L_{\psi} \cdot j^{\infty} \phi_{\left.\right|_{x}}=j^{\infty} \psi_{\left.\right|_{\tau\left(\left.j^{\infty} \varphi\right|_{x}\right)}} \cdot j^{\infty} \varphi_{\left.\right|_{x}}
$$

Note that projecting the right action onto the source coordinate gives

$$
\sigma\left(R_{\psi} \cdot j^{\infty} \phi_{\left.\right|_{x}}\right)=\psi(x)
$$


and so we can extend this action to $\widetilde{\mathcal{G}}_{\infty}$ by

$$
R_{\psi} \cdot\left(z_{\left.\right|_{x}}^{(\infty)}, j^{\infty} \phi_{\left.\right|_{x}}\right):=\left(j^{\infty} \psi_{\left.\right|_{x}} \cdot z_{\left.\right|_{x}}^{(\infty)},\left.j^{\infty} \varphi_{\left.\right|_{x}} \cdot j^{\infty} \psi^{-1}\right|_{\psi(x)}\right)
$$

for all $x$ in the domain of definition of $\psi$. The target map $\tilde{\tau}: \widetilde{\mathcal{G}}_{\infty} \rightarrow J^{\infty}(\mathcal{E})$ provides a complete collection of all scalar invariants of this action:

$$
\begin{aligned}
R_{\psi}^{*} \tilde{\tau}\left(z_{\mid x}^{(\infty)}, j^{\infty} \phi_{\left.\right|_{x}}\right) & =\tau\left(\left.j^{\infty} \psi_{\left.\right|_{x}} \cdot z^{(\infty)}\right|_{x},\left.j^{\infty} \varphi_{\left.\right|_{x}} \cdot j^{\infty} \psi^{-1}\right|_{\psi(x)}\right) \\
& =\left.j^{\infty} \varphi_{\left.\right|_{x}} \cdot j^{\infty} \psi^{-1}\right|_{\psi(x)} \cdot\left(\left.j^{\infty} \psi_{\left.\right|_{x}} \cdot z^{(\infty)}\right|_{x}\right) \\
& =\left(\left.j^{\infty} \varphi_{\left.\right|_{x}} \cdot j^{\infty} \psi^{-1}\right|_{\psi(x)} \cdot j^{\infty} \psi_{\left.\right|_{x}}\right) \cdot z_{\left.\right|_{x}}^{(\infty)}=j^{\infty} \phi_{\left.\right|_{x}} \cdot z_{\left.\right|_{x}}^{(\infty)} \\
& =\tilde{\tau}\left(\left.z\right|_{x} ^{(\infty)}, j^{\infty} \phi_{\left.\right|_{x}}\right) .
\end{aligned}
$$

This also means that the pull-back of any differential form $\omega$ on $J^{\infty}(\mathcal{E})$ by the target map is invariant under this action:

$$
R_{\psi}^{*}\left(\tilde{\tau}^{*} \omega\right)=\left(\tilde{\tau} \circ R_{\psi}\right)^{*} \omega=\tilde{\tau}^{*} \omega
$$

In [42] a basis, $\left\{\mu_{K}^{i}\right\}_{K \in \mathbb{N}_{0}^{n}, 1 \leq i \leq n}$, for the contact co-distribution on the diffeomorphism groupoid $\mathcal{D}_{\infty}$, that is right invariant was constructed. Naturally, these contact forms are called the Maurer-Cartan forms of the pseudo-group $\mathcal{D}$. The form $\mu_{K}^{i}$ agrees with the standard contact form $\Upsilon_{K}^{i}=d X_{K}^{i}-X_{K, j}^{i} d x^{j}$ on the identity section $\mathbb{1}$ of $\mathcal{D}_{\infty} \rightarrow \mathcal{X}$, and each $\mu_{K}^{i}$ is a linear combination of $\Upsilon_{J}^{j}$ for $|J| \leq|K|$ (and conversely). When we restrict the Maurer-Cartan forms to a sub-groupoid $\mathcal{G}_{\infty} \subset \mathcal{D}_{\infty}$, we obtain certain linear dependencies among the $\mu_{K}^{i}$. The important discovery, made in [42], is that these are given by

$$
\left.\frac{\partial F\left(x, X^{(q)}\right)}{\partial X_{K}^{i}}\right|_{\widetilde{\mathbb{1}}} \mu_{K}^{i}=0,
$$

where restriction to $\widetilde{\mathbb{1}}$ means first restricting to the identity section and then replacing all source coordinates $x$ by target coordinates $X$. 
Remark 4.2.1. The structure equations, or the formulas for $d \mu_{K}^{i}$, are rather complicated expressions but we will mostly be interested in their top order terms. The entire equations are

$$
d \mu_{K}^{i}=\sum_{1 \leq j \leq n} \omega^{j} \wedge \mu_{K, j}^{i}+\sum_{\substack{L+M=K \\
|M| \geq 1}}\left(\begin{array}{c}
K \\
L
\end{array}\right) \sum_{1 \leq j \leq n} \mu_{L, j}^{i} \wedge \mu_{M}^{j} .
$$

Where we refer to $\sum_{1 \leq j \leq n} \omega^{j} \wedge \mu_{K, j}^{i}$ as the top order term, $L+M$ is the componentwise addition of multi-indices in $\mathbb{N}_{0}^{n}$ and

$$
\left(\begin{array}{c}
K \\
L
\end{array}\right)=\frac{K !}{L ! M !}
$$

The Maurer-Cartan forms embed naturally into the pull-back bundle $\widetilde{\mathcal{G}}_{\infty} \rightarrow$ $J^{\infty}(\mathcal{E})$. In fact, the space of one-forms on $\widetilde{\mathcal{G}}_{\infty}$ is a direct sum of two right-invariant (recall (4.7)) subspaces. Complementing the Maurer-Cartan forms in this direct sum are the one-forms on $J^{\infty}(\mathcal{E})$, pulled back to $\widetilde{\mathcal{G}}_{\infty}$ by the target map $\tilde{\tau}$. This direct sum imbues the differential forms on $\widetilde{\mathcal{G}}_{\infty}$ with a bigration. We correspondingly split the exterior derivative on $\widetilde{\mathcal{G}}_{\infty}$ into a group- and jet-component,

$$
d=d_{G}+d_{J},
$$

where $d_{G}$ increases the group-grade and $d_{J}$ increases the jet-grade. Notice that since $\psi \in \mathcal{G}$ acts on the group and submanifold jet coordinates separately, $R_{\psi}$. $\left(\left.z\right|_{x} ^{(\infty)}, j^{\infty} \phi_{\left.\right|_{x}}\right)=\left(j^{\infty} \psi_{\left.\right|_{x}} \cdot z_{\left.\right|_{x}}^{(\infty)},\left.j^{\infty} \varphi_{\left.\right|_{x}} \cdot j^{\infty} \psi^{-1}\right|_{\psi(x)}\right)$, the group and jet components of the derivative of any invariant form are again invariant. The differential one-forms on $J^{\infty}(\mathcal{E})$ further divide into horizontal and contact forms with bases

$$
\left\{d x^{1}, \ldots, d x^{n}\right\} \quad \text { and } \quad\left\{d u_{J}^{\alpha}-u_{J, i}^{\alpha} d x^{i}\right\}_{J \in \mathbb{N}_{0}^{n}, 1 \leq \alpha \leq m}
$$

respectively. The jet-differential $d_{J}$ then splits accordingly into a horizontal and 
vertical (submanifold contact) component,

$$
d_{J}=d_{H}+d_{V}
$$

Since the right action of $\mathcal{G}$ on $\widetilde{\mathcal{G}}_{\infty}$ obviously preserves horizontal and vertical forms (being horizontal), as well as the Maurer-Cartan forms, these various differentials of invariant forms on $\widetilde{\mathcal{G}}_{\infty}$ are still invariant.

Let us define an operator, $\gamma_{J}$, that takes a general differential form on the pullback bundle $\widetilde{\mathcal{G}}_{\infty} \rightarrow J^{\infty}(\mathcal{E})$, that is written in terms of the Maurer-Cartan forms, horizontal forms and submanifold jet contact forms, and equates all MaurerCartan forms to zero. Since the right action preserves the group and submanifold jet components of $\tilde{\tau}^{*} \omega$ we have that $\gamma_{J} \tilde{\tau}^{*} \omega$ is invariant. We call the operator

$$
\lambda:=\gamma_{J} \tilde{\tau}^{*}
$$

the lift operator. We say that a differential form $\Omega$ on $\widetilde{\mathcal{G}}_{\infty}$ is concentrated on $J^{\infty}(\mathcal{E})$ if $\gamma_{J}(\Omega)=\Omega$.

\subsection{Recurrence formula}

In the calculus of moving frames for Lie pseudo-groups, the recurrence formula plays a fundamental role. We now deduce it for horizontal actions, but the proofs for general Lie pseudo-group actions are identical.

Let $\Phi_{\varepsilon}=\left(\Phi_{\varepsilon}^{1}, \ldots, \Phi_{\varepsilon}^{p}\right)$ be a one parameter family of diffeomorphisms from $\mathcal{G}$ with $\Phi_{0}$ being the identity. It generates a vector field on $\mathcal{X}$,

$$
\mathbf{v}(x):=\left.\frac{d}{d \varepsilon}\right|_{\varepsilon=0} \Phi_{\varepsilon}^{i}(x) \frac{\partial}{\partial x^{i}}=\zeta^{i}(x) \frac{\partial}{\partial x^{i}},
$$

whose components, $\zeta^{i}$, satisfy 4.3). By prolongation, we have the flow $\varepsilon \mapsto$ $\tilde{\tau}\left(z_{\mid x}^{(\infty)}, j^{\infty} \Phi_{\left.\varepsilon\right|_{x}}\right)$ on $J^{\infty}(\mathcal{E})$ generating the vector field $\widehat{\mathbf{v}}^{\infty}\left(z_{\left.\right|_{x}}^{(\infty)}\right)$. The explicit formulas for the different components of $\widehat{\mathbf{v}}^{\infty}$ can be given by a simple recurrence 
relation, see [38].

The lift of the flow $\Phi_{\varepsilon}$ is the flow on $\mathcal{G}_{\infty}$ given by $\varepsilon \mapsto j^{\infty}{ }_{{ }_{X}} \Phi_{\varepsilon} \cdot j^{\infty} \psi_{\left.\right|_{x}}$ for $j^{\infty} \psi_{\left.\right|_{x}} \in \mathcal{G}_{\infty}$ with $\tau\left(j^{\infty} \psi_{\left.\right|_{x}}\right)=X$ in the domain of definition of $\Phi_{\varepsilon}$. Note that it is tangent to the source fibers $\sigma^{-1}(x)$. This flow is obviously right-invariant and analyzing it at the identity section we find the vector field that it generates: $\widehat{\mathbf{V}}^{\infty}=\sum \zeta_{K}^{i}(X) \frac{\partial}{\partial X_{K}^{i}}$. Note that, by definition, $\widehat{\mathbf{V}}^{\infty}$ and $\widehat{\mathbf{v}}^{\infty}$ are related by the push-forward at the identity, $\tilde{\tau}_{*}\left(\left.\widehat{\mathbf{V}}^{\infty}\right|_{\mathbb{1}}\right)=\widehat{\mathbf{v}}^{\infty}$, where, implicitly, we have transferred $\widehat{\mathbf{V}}^{\infty}$ from $\mathcal{G}_{\infty}$ to $\widetilde{\mathcal{G}}_{\infty}$.

We would like to know what happens when we take the exterior derivative of a lifted form $\lambda(\omega)$, where $\omega$ is a differential form on $J^{\infty}(\mathcal{E})$. It is relatively easy (by an examination of $\lambda$ ) to see that $d_{J} \lambda(\omega)=\lambda(d \omega)$. The group component $d_{G} \lambda$ is more difficult to establish, but we have, for a lifted Lie algebroid vector field $\widehat{\mathbf{V}}^{\infty}$, since $\tilde{\tau}_{*}\left(\left.\widehat{\mathbf{V}}^{\infty}\right|_{\mathbb{1}}\right)=\widehat{\mathbf{V}}^{\infty}$, at the identity section,

$$
\widehat{\mathbf{V}}^{\infty}(\lambda(\omega))=\widehat{\mathbf{V}}^{\infty}\left(\gamma_{J} \tilde{\tau}^{*} \omega\right)=\gamma_{J} \widehat{\mathbf{V}}^{\infty}\left(\tilde{\tau}^{*} \omega\right)=\gamma_{J} \tilde{\tau}^{*}\left(\widehat{\mathbf{v}}^{\infty}(\omega)\right)
$$

On the other hand, we have by Cartan's formula

$$
\left.\left.\left.\widehat{\mathbf{V}}^{\infty}(\lambda(\omega))=\widehat{\mathbf{V}}^{\infty}\right\lrcorner d \lambda(\omega)+d\left(\widehat{\mathbf{V}}^{\infty}\right\lrcorner \lambda(\omega)\right)=\widehat{\mathbf{V}}^{\infty}\right\lrcorner d_{G} \lambda(\omega),
$$

since $\widehat{\mathbf{V}}^{\infty}$ only has group components (tangent to the $\tilde{\sigma}$-fibers). Now, $\left.\widehat{\mathbf{V}}^{\infty}\right\lrcorner$ $\left.d_{G} \lambda(\omega)=\widehat{\mathbf{V}}^{\infty}\right\lrcorner \sum\left(\mu_{K}^{i} \wedge \Omega_{i}^{K}\right)$ is an invariant differential form on $\widetilde{\mathcal{G}}_{\infty}$, where $\Omega_{i}^{K}$ are some differential forms concentrated on $J^{\infty}(\mathcal{E})$. Evaluating it at the identity section gives

$$
\widehat{\mathbf{V}}^{\infty}(\lambda(\omega))=\sum \zeta_{K}^{i}(X) \Omega_{i}^{K} .
$$

Both (4.12) and (4.13) are invariant differential forms that agree at the identity section, but this means they must agree everywhere. Writing $\gamma_{J} \tilde{\tau}^{*}\left(\widehat{\mathbf{v}}^{\infty}(\omega)\right)=$ $\sum \zeta_{K}^{i}(X) \widetilde{\Omega}_{i}^{K}$ (note that $\widehat{\mathbf{v}}^{\infty}$ is a linear function of the components $\zeta_{K}^{i}$ ), we have, 
for every vector field in the Lie algebroid of $\mathcal{G}$ that

$$
\sum \zeta_{K}^{i}(X) \widetilde{\Omega}_{i}^{K}=\sum \zeta_{K}^{i}(X) \Omega_{i}^{K}
$$

and hence $\widetilde{\Omega}_{i}^{K}=\Omega_{i}^{K}$. This gives the original recurrence formula, where on the left hand side we need to replace every $\lambda\left(\zeta_{K}^{i}\right)$ by $\mu_{K}^{i}$ :

$$
\lambda\left(\widehat{\mathbf{v}}^{\infty}(\omega)\right)=d_{G} \lambda(\omega)
$$

This is the original derivation of the recurrence formula for pseudo-groups from [43. But we can also calculate directly at the identity section,

$$
\left.\widehat{\mathbf{V}}^{\infty}(\lambda(\omega))\right|_{\mathbb{1}}=\left.\sum \zeta_{K}^{i}(X)\left(\frac{\partial(\lambda(\omega))}{\partial X_{K}^{i}}\right)\right|_{\mathbb{1}}
$$

to see that $\left.\Omega_{i}^{K}\right|_{\mathbb{1}}=\left.\left(\frac{\partial(\lambda(\omega))}{\partial X_{K}^{i}}\right)\right|_{\mathbb{1}}$. Each $\Omega_{i}^{K}$ is invariant on $\widetilde{\mathcal{G}}_{\infty}$ and so we can deduce that, in general,

$$
\widehat{\mathbf{V}}^{\infty}(\lambda(\omega))=\sum \zeta_{K}^{i}(X) \lambda\left(\left.\frac{\partial(\lambda(\omega))}{\partial X_{K}^{i}}\right|_{\mathbb{1}}\right)
$$

We can then write the recurrence formula

$$
d_{G} \lambda(\omega)=\sum \mu_{K}^{i} \wedge \lambda\left(\left.\frac{\partial(\lambda(\omega))}{\partial X_{K}^{i}}\right|_{\mathbb{1}}\right)
$$

For our purpose of proving the termination of Cartan's equivalence method, we will work with the recurrence formula in this form. Notice that 4.15 is a kind of group parameter linearization of $\lambda(\omega)$, e.g. if $\omega=u_{J}^{\alpha}$ we have

$$
d_{G} \lambda u_{J}^{\alpha}=d_{G} U_{J}^{\alpha}=\sum \lambda\left(\left.\frac{\partial U_{J}^{\alpha}}{\partial X_{K}^{i}}\right|_{\mathbb{1}}\right) \mu_{K}^{i}
$$


The invariant horizontal forms $\omega^{i}:=\lambda\left(d x^{i}\right)$ are especially important. We have

$$
\omega^{i}=\lambda\left(d x^{i}\right)=\gamma_{J} \tilde{\tau}^{*} d x^{i}=\gamma_{J} d X^{i}=\gamma_{J}\left(X_{j}^{i} d x^{j}-\Upsilon^{i}\right)=X_{j}^{i} d x^{j}
$$

Another important identity is

$$
d_{H} U_{J}^{\alpha}=U_{J, i}^{\alpha} \omega^{i}
$$

which can be deduced by computing

$$
\begin{aligned}
& d_{H} \lambda\left(u_{J}^{\alpha}\right)+d_{V} \lambda\left(U_{J}^{\alpha}\right)=d_{J} \lambda\left(u_{J}^{\alpha}\right)=\lambda\left(d u_{J}^{\alpha}\right) \\
& =\lambda\left(u_{J, i}^{\alpha} d x^{i}\right)+\lambda(\text { vertical forms }) \\
& =U_{J, i}^{\alpha} \omega^{i}+\text { vertical forms }
\end{aligned}
$$

and comparing horizontal parts.

Combining (4.16) and (4.17) we have

$$
d U_{J}^{\alpha}=U_{J, i}^{\alpha} \omega^{i}+\sum \lambda\left(\left.\frac{\partial U_{J}^{\alpha}}{\partial X_{K}^{i}}\right|_{\mathbb{1}}\right) \mu_{K}^{i}+\text { contact forms on } J^{\infty}(\mathcal{E}) .
$$




\section{Chapter 5}

\section{Equivalence of sections}

Let $\mathcal{G}$ be a Lie pseudo-group that acts horizontally on the bundle $\mathcal{E}=\mathcal{X} \times \mathcal{U} \stackrel{\pi}{\rightarrow} \mathcal{X}$ as described in previous sections. In this chapter we start exploring the problem of local equivalence of sections of $\mathcal{E}$. That is, for two sections of $\mathcal{E}$, that are the graphs of two locally defined functions from $\mathcal{X}$ to $\mathcal{U}, u$ and $\bar{u}$, is there a $\varphi \in \mathcal{G}$ such that (recall that we restrict to "first order actions" for simplicity)

$$
j^{1} \varphi_{\left.\right|_{x}} \cdot(x, u(x))=(\varphi(x), \bar{u}(\varphi(x)),
$$

for all $x$ in some open subset of $\operatorname{dom} u$, and how do we characterize all such congruences? We saw in the previous chapter how this congruence problem models the equivalence of sections of tensor-bundles, ordinary differential equations and Lagrangians. One approach to finding all congruence maps $\varphi$ would be to write down the defining equations of $\mathcal{G}$ and the differential equation (5.1), and try to complete this collection of equations to involution via our general Algorithm 3.2.6. This is not a very attractive route as the equations (5.1) are, in general, extremely complicated. In this chapter we shall first develop a group modification of Algorithm 3.2.6, where we exchange the standard contact forms for the MaurerCartan forms. We then introduce an object called the equivariant moving frame for pseudo-groups, which, in conjunction with the recurrence formula will be our 
fundamental tool, both for the theory as well as in practical applications in later sections. Section 5.3 gives a proof of a pseudo-group analog of the result for Lie groups that underlies the congruence problem in the finite dimensional case. In the final section we collect all our findings to prove termination of Cartan's equivalence method in the horizontal case.

For a locally defined function $u$, we shall denote the section $x \mapsto(x, u(x))$ by $j^{0} u$, and its higher jets by $j^{p} u, 0 \leq p \leq \infty$.

\subsection{A group modification of Algorithm 3.2.6}

Given sections $x \rightarrow(x, u(x))$ and $\bar{x} \rightarrow(\bar{x}, \bar{u}(\bar{x}))$ of $\mathcal{E}$ we want to characterize those $\varphi \in \mathcal{G}$ that map one to the other. Such a $\varphi$ must first of all be a solution to the defining equations of $\mathcal{G}, 4.1$, and second of all it must solve the differential equation

$$
\left(\varphi(x), U_{\left.\right|_{u}}\right)=j^{1} \varphi_{\left.\right|_{x}} \cdot(x, u(x))=(\varphi(x), \bar{u}(\varphi(x)))
$$

where $U_{\left.\right|_{u}}$ denotes the restriction of $U$ to the jets of the section determined by $u$, i.e. $j^{\infty} u_{\left.\right|_{x}}$. Using the jet-coordinate notation, we can write the extra condition (5.2) as

$$
\bar{u}(X)=U_{\left.\right|_{u}} .
$$

Denote the differential equation obtained by adjoining (5.3) to 4.1) by $\mathcal{G}_{\infty}^{u \bar{u}} \subset$ $\mathcal{G}_{\infty}$. We will eventually show that Cartan's equivalence method terminates when Algorithm 3.2.6, from Section 3.2, does for $\mathcal{G}_{\infty}^{u \bar{u}}$. Now, instead of prolonging (5.3) using the standard total derivative $D_{x^{i}}$, we will use differentiation with respect to the target coordinates, $D_{X^{i}}$, dual to the lifted horizontal forms $\omega^{i}=X_{j}^{i} d x^{j}$. The two sets of differential operators are related by an invertible linear transformation and so these two ways of prolonging give algebraically equivalent representations of the differential equation $\mathcal{G}_{\infty}^{u \bar{u}}$. Applying $\left(D_{X}\right)^{J}=\frac{\partial^{|J|}}{\partial \omega^{J}}$ to 5.2 , the prolonged 
equations now take the form

$$
\bar{u}_{J}^{\alpha}(X)=\left.U_{J}^{\alpha}\right|_{u} .
$$

We wish to develop a version of Algorithm 3.2 .6 for $\mathcal{G}^{u \bar{u}}$ where we replace the standard contact forms, $\Upsilon_{K}^{i}$, used in Chapter 3 by the Maurer-Cartan forms $\mu_{K}^{i}$. We have two sets of defining equations, (4.1) and (5.4), and restricting the contact structure of $J^{\infty}(\mathcal{X} \times \mathcal{X})$ to these, using the $\mu_{K}^{i}$, gives (by equation 4.9$)$ and the recurrence formula $(4.15)$ )

$$
\begin{aligned}
0 & =d F=d_{H} F+d_{G} F=d_{H} F+\left.\frac{\partial F}{\partial X_{K}^{i}}\right|_{\widetilde{\mathbb{1}}} \mu_{K}^{i}, \\
0 & =d_{H}\left(\bar{u}_{J}^{\alpha}(X)-U_{\left.J\right|_{u}}^{\alpha}\right)+d_{G}\left(\bar{u}_{J}^{\alpha}(X)-\left.U_{J}^{\alpha}\right|_{u}\right) \\
& =\left(\bar{u}_{J, i}^{\alpha}(X) \omega^{i}-U_{J,\left.i\right|_{u}}^{\alpha} \omega^{i}\right)+\bar{u}_{J, i}^{\alpha}(X) \mu^{i}-\lambda\left(\frac{\left.\partial U_{J}^{\alpha}\right|_{u}}{\left.\partial X_{K}^{i}\right|_{\mathbb{1}}}\right) \mu_{K}^{i} .
\end{aligned}
$$

Notice that these equations have almost exactly the same form as if we had used the basis of standard contact forms $\Upsilon_{K}^{i}$ and the total derivatives $D_{x^{i}}$. The only difference is that the above equations are all evaluated at the identity section and source coordinates have been replaced by target coordinates. That is, replacing every $\mu_{K}^{i}$ by $\Upsilon_{K}^{i}$ in $(5.5)$ will give the corresponding relations for the standard contact forms (at the identity section).

Algorithm 3.2.6 is built on the exterior derivative, and one could presume that working with the Maurer-Cartan forms instead of the standard contact forms might introduce extra complications. However, we have (cf. Remark 4.2.1)

$$
d \mu_{K}^{i}=\omega^{i} \wedge \mu_{K, j}^{i}+\text { lower order forms }
$$

and so, at the top order, $d \mu_{K}^{i}$ and $d \Upsilon_{K}^{i}$ have the same structure (interchanging $\omega^{i}$ and $\left.d x^{i}\right)$. Since, in the search for asymptotically regular coordinates in Algorithm 3.2.6. we project onto the space of contact forms of top order, equation 5.6 means that we can just as well work with the Maurer-Cartan forms and the $\omega^{i}$. 
For example, denoting by $\mathcal{G}_{1}^{u \bar{u}}$ the first order determining equations for $\mathcal{G}_{\infty}^{u \bar{u}}$, the number of parametric derivatives of class 1 and order 1 in $\mathcal{G}_{1}^{u \bar{u}}$ is equal to

$$
\text { the rank of } \left.\left.\left\{\gamma_{1}\left(D_{X^{1}}\right\lrcorner d \mu^{i}\right)\right|_{\mathcal{G}_{1}^{u \bar{u}}}\right\}_{i=1, \ldots, n} \text {. }
$$

Hence, combining (5.6) with the fact that the linear dependencies between the Maurer-Cartan forms given by (5.5) are isomorphic to ones we obtain when using the standard contact forms $\Upsilon_{K}^{i}$, we notice that we will obtain the same ranks during the implementation of Algorithm 3.2 .6 whether we are using $D_{X^{i}}$ and $\mu_{K}^{i}$ or $D_{x^{i}}$ and $\Upsilon_{K}^{i}$. Notice also that since $\mu_{K}^{i}$ agrees with $\Upsilon_{K}^{i}$ on the identity section, and the collection $\left\{\mu_{K}^{i}\right\}_{|K| \leq q}$ is an invertible linear combination of the $\left\{\Upsilon_{K}^{i}\right\}_{|K| \leq q}$ for each $q$, when searching for integrability conditions and prolonged equations for $\mathcal{G}^{u \bar{u}}$ as in Remark 3.2.4 we can just as well use $\mu_{K}^{i}$ as $\Upsilon_{K}^{i}$. This includes ignoring exterior derivatives of $\mu_{K}^{i}$ for $X_{K}^{i}$ principal (if no integrability conditions are found going from $\mathcal{G}_{|K|}^{u \bar{u}}$ to $\mathcal{G}_{|K|, 1}^{u \bar{u}}$ ). This is because $d \Upsilon_{K}^{i}$ can be ignored and $\mu_{K}^{i}$ explicitly depends on $\Upsilon_{K}^{i}$ since they agree on the identity section.

By our blanket, simplifying assumption, $U^{\alpha}$ depends on first order jets and so the lowest order determining equations of $\mathcal{G}_{\infty}^{u \bar{u}}$ are first order. Let us also assume, purely for simplicity, that $\mathcal{G}$ is determined by first order equations and has regularity order 1. Algorithm 3.2.6 for the system $\mathcal{G}_{\infty}^{u \bar{u}}$ now takes the following form. Recall that $\mathcal{G}_{1,1}^{u \bar{u}}$ is the first prolongation of $\mathcal{G}_{1}^{u \bar{u}}$ and $\mathcal{G}_{1,1}^{u \bar{u}(1)}$ is its projection to first order.

\section{Algorithm 5.1.1.}

(a) Compute all the exterior derivatives $d \mu^{i}, i=1, \ldots, n$, and restrict to $\mathcal{G}_{1}^{u \bar{u}}$ using the equations (5.5). The coefficients of the purely horizontal parts $\omega^{i} \wedge \omega^{j}$ are the cross-derivatives and prolongations when going from $\mathcal{G}_{1}^{u \bar{u}}$ to $\mathcal{G}_{1,1}^{u \bar{u}}$. If $\mathcal{G}_{1,1}^{u \bar{u}^{(1)}} \subsetneq \mathcal{G}_{1}^{u \bar{u}}$, replace $\mathcal{G}_{1}^{u \bar{u}}$ by $\mathcal{G}_{1,1}^{u \bar{u}^{(1)}}$ and repeat. 
(b) When we stop finding integrability conditions we test for involution by computing the ranks of collections of the form

$$
\left.\left\{\gamma_{1}\left(a_{1}^{j} D_{X^{j}}\right\lrcorner d \mu^{i}\right)_{\left.\right|_{1} ^{u \bar{u}}}\right\}_{i=1, \ldots, n},
$$

and counting the number of parametric derivatives of order 2 . If the test fails, then compute $d \mu_{j}^{i}$, for all parametric $X_{j}^{i}$, restricted to $\mathcal{G}_{1,1}^{u \bar{u}}$, search for integrability conditions, and so on.

Remark 5.1.2. Actually, by the general structure equations for the MaurerCartan forms on the diffeomorphism groupoid $\mathcal{D}_{\infty}$, we have

$$
d \omega^{i}=-d \mu^{i}
$$

so in step (a) of Algorithm 5.1.1 we could also have computed $d \omega^{i}$ instead of $d \mu^{i}$.

Remark 5.1.3. Recall Remark 3.2.7, where we mentioned that computing the exterior derivatives of contact forms on differential equations is usually not the most practical method of prolongation. In the next section, we introduce the moving frame for pseudo-groups and then we introduce an algorithm for constructing it. In order to prove the termination of that algorithm we shall need Algorithm 5.1 .1 .

Although we will primarily be interested in the congruence of sections, Algorithm 5.1.1 can, of course, be used to complete any Lie pseudo-group (that is regular in the sense of Definition 3.0.6 to involution. We give a (very) simple example of how this works.

Example 5.1.4. Consider the pseudo-group $\mathcal{G}$, with determining equations

$$
X_{y}=0, \quad Y_{y}=X_{x}
$$


Prolonging once, we obtain the equations

$$
X_{x y}=X_{y y}=Y_{y y}=0, \quad Y_{y x}=X_{x x}
$$

Therefore $\mathcal{G}_{1,1}^{(1)}=\mathcal{G}_{1}$ and $r^{(2)}=\left|\left\{X_{x x}, Y_{x x}\right\}\right|=2$. Restricting the first order Maurer-Cartan forms on $\mathcal{D}_{\infty}$ to $\mathcal{G}_{1}$ gives

$$
\mu_{y}^{x}=0, \quad \mu_{y}^{y}=\mu_{x}^{x}
$$

To test whether $\mathcal{G}_{1}$ is involutive we first maximize the rank of

$$
\left.\left\{\gamma_{1}\left(\left(a \frac{\partial}{\partial \omega^{x}}+b \frac{\partial}{\partial \omega^{y}}\right)\right\lrcorner d \mu^{i}\right)\right\}_{i=x, y}
$$

We have

$$
\begin{aligned}
& d \mu^{x}=\omega^{x} \wedge \mu_{x}^{x}+\omega^{y} \wedge \mu_{y}^{x}=\omega^{x} \wedge \mu_{x}^{x} \\
& d \mu^{y}=\omega^{x} \wedge \mu_{x}^{y}+\omega^{y} \wedge \mu_{y}^{y}=\omega^{x} \wedge \mu_{x}^{y}+\omega^{y} \wedge \mu_{x}^{x}
\end{aligned}
$$

and maximizing the rank of $(5.9)$ then means maximizing the rank of the set

$$
\left\{a \mu_{x}^{x}, a \mu_{x}^{y}+b \mu_{x}^{x}\right\}
$$

which is equivalent to maximizing the rank of the matrix

$$
\left[\begin{array}{ll}
a & 0 \\
b & a
\end{array}\right]
$$

This matrix has maximum rank 2 for example when $(a, b)=(1,0)$, so $\tilde{s}_{1}^{(1)}=2$. And since the matrix actually has full rank we have $\tilde{s}_{2}^{(1)}=0$. Cartan's test is satisfied since

$$
2=r^{(2)}=\sum i \tilde{s}_{i}^{(1)}=1 \cdot 2+2 \cdot 0=2 .
$$


From our work we can also see that the given coordinates provide $\delta$-regular ones, since $(1,0)$ and $(0,1)$ give maximum ranks leading to the reduced Cartan characters. Again, in this simple example, this is obvious, but this involutivity test will prove that it's worth its salt before long.

As mentioned above, Algorithm 5.1.1 is not practical for more substantial problems. The next section introduces a fundamental computational tool called the equivariant moving frame for pseudo-groups, as we edge a little bit closer to a practical algorithm for solving congruence problems.

\subsection{The equivariant moving frame}

The lifted submanifold jet coordinate functions $U_{J}^{\alpha}=\tau^{*} u_{J}^{\alpha}$ are invariant under the right action of $\mathcal{G}$ on the bundle $\widetilde{\mathcal{G}}_{\infty} \rightarrow J^{\infty}(\mathcal{E})$, and form a complete collection of invariants for this action. The equivariant moving frame, [43], is an object that computes the invariants of the prolonged action of $\mathcal{G}$ on the submanifold jet bundle $J^{\infty}(\mathcal{E})$. Its definition is simple enough.

Definition 5.2.1. A (local) moving frame is a (local) right-equivariant section, $\rho$, of $\widetilde{\mathcal{G}}_{\infty} \stackrel{\tilde{\sigma}}{\rightarrow} J^{\infty}(\mathcal{E})$, i.e.

$$
\rho\left(\left.\psi \cdot z\right|_{x} ^{(\infty)}\right)=R_{\psi} \cdot \rho\left(\left.z\right|_{x} ^{(\infty)}\right),
$$

for all $\psi \in \mathcal{G}$ with $x \in \operatorname{dom} \psi$.

We can write 5.10 as

$$
\rho \circ \psi=R_{\psi} \circ \rho
$$

and so right-equivariance is the property of a section of $\widetilde{\mathcal{G}}_{\infty} \rightarrow J^{\infty}(\mathcal{E})$ that its image is invariant under the right action of $\mathcal{G}$ on $\widetilde{\mathcal{G}}_{\infty}$.

Recall that we denoted the source map on $\widetilde{\mathcal{G}}_{\infty} \rightarrow J^{\infty}(\mathcal{E})$ by $\tilde{\sigma}$ and we shall denote the bundle maps $\widetilde{\mathcal{G}}_{t} \rightarrow \widetilde{\mathcal{G}}_{p}$ by $\tilde{\sigma}_{p}^{t}, 0 \leq p<t \leq \infty$. Recall also that the bundle maps on the submanifold jet spaces are denoted $\pi_{p}^{t}: J^{t}(\mathcal{E}) \rightarrow J^{p}(\mathcal{E})$. A 
moving frame pulls any invariant object on $\widetilde{\mathcal{G}}_{\infty}$ back to an invariant object on $J^{\infty}(\mathcal{E})$ : Let $\Omega$ be an invariant differential form on $\widetilde{\mathcal{G}}_{\infty}$ and let $\omega=\rho^{*} \Omega$ be its pull-back on $J^{\infty}(\mathcal{E})$. Then, for $\psi \in \mathcal{G}$,

$$
\psi^{*} \omega=\psi^{*} \rho^{*} \Omega=(\rho \circ \psi)^{*} \Omega=\left(R_{\psi} \circ \rho\right)^{*} \Omega=\rho^{*} R_{\psi}^{*} \Omega=\rho^{*} \Omega=\omega,
$$

by invariance of $\Omega$. In particular, $\rho^{*} U_{J}^{\alpha}$ are a complete collection of invariants of the action of $\mathcal{G}$ on $J^{\infty}(\mathcal{E})$. Generalizing a little bit, we define a partial moving frame. (Right-equivariance of a subspace of $\widetilde{\mathcal{G}}_{\infty}$ just means that it is preserved by the right action.)

Definition 5.2.2. Let $\mathcal{S} \subset J^{\infty}(\mathcal{E})$ and denote by $\mathcal{G}_{\infty}^{\mathcal{S}}$ the jets $j^{\infty} \varphi_{\left.\right|_{x}}, x \in \pi_{0}^{\infty}(\mathcal{S})$, that preserve $\mathcal{S}$. A (local) partial moving frame on $\mathcal{S}$ is a fibered subspace, $\mathcal{B} \stackrel{\tilde{\sigma}}{\rightarrow} \mathcal{S}$ of $\widetilde{\mathcal{G}}_{\infty} \rightarrow J^{\infty}(\mathcal{E})$, that is right-equivariant under the action of $\mathcal{G}_{\infty}^{\mathcal{S}}$. The set $\mathcal{S}$ is called the domain of definition of $\mathcal{B}$.

Remark 5.2.3. We can define a partial moving frame of any order $p$ as a rightequivariant subspace (under $\mathcal{G}_{p}^{\mathcal{S}}$ ), $\mathcal{B}_{p} \rightarrow \mathcal{S}$, of the bundle $\widetilde{\mathcal{G}}_{p} \rightarrow J^{p}(\mathcal{E})$. Denoting its preimage in $\widetilde{\mathcal{G}}_{\infty}$ by $\widetilde{\mathcal{B}}_{p}:=\left(\tilde{\sigma}_{p}^{\infty}\right)^{-1}\left(\mathcal{B}_{p}\right)$ we notice that $\widetilde{\mathcal{B}}_{p}$ is a partial moving frame in $\widetilde{\mathcal{G}}_{\infty}$.

Remark 5.2.4. Definition 5.2 .2 is more general than previous definitions of partial moving frames which are usually only defined as fibered subspaces over a base $\mathcal{S}$ where $\mathcal{S}$ is assumed to be locally $\mathcal{G}$-invariant. Our definition is strictly more general as (locally) $\mathcal{G}^{\mathcal{S}}=\mathcal{G}$ for locally $\mathcal{G}$-invariant $\mathcal{S}$. Furthermore, our key result in the next subsection allows us to extend the moving frame technique to equivalence problems for sections that have until now remained outside the scope of the equivariant moving frame.

Just like a moving frame pulls invariant objects on $\widetilde{\mathcal{G}}_{\infty}$ to invariant objects on $J^{\infty}(\mathcal{E})$, restricting any invariant object on $\widetilde{\mathcal{G}}_{\infty}$ to a partial moving frame $\mathcal{B} \rightarrow \mathcal{S}$ gives an invariant object on $\mathcal{B}$ under $\mathcal{G}^{\mathcal{S}}$. 
There turns out to be a practical construction available for a partial moving frame. It is equivalent to a choice of a cross-section to the orbits of the action of $J^{\infty}(\mathcal{E})$. A cross-section $\mathcal{K} \subset J^{\infty}(\mathcal{E})$ to the orbits is a (connected) subspace such that if an orbit intersects $\mathcal{K}$, it does so at a unique point and transversally. Given such a cross-section we can construct a moving frame as follows. Let $z_{x}^{(\infty)} \in$ $J^{\infty}(\mathcal{E})$ and assume the orbit through $z_{\left.\right|_{x}}^{(\infty)}$ intersects $\mathcal{K}$. Then define the fiber over $z_{x}^{(\infty)} \in J^{\infty}(\mathcal{E})$ to be the collection of $j^{\infty} \phi_{\left.\right|_{x}} \in \widetilde{\mathcal{G}}_{\infty}$ such that

$$
j^{\infty} \phi_{\left.\right|_{x}} \cdot z_{x}^{(\infty)} \in \mathcal{K}
$$

If the action is free on $J^{\infty}(\mathcal{E})$, then this partial moving frame reduces to a moving frame since then $j^{\infty} \phi_{\left.\right|_{x}} \cdot z_{\left.\right|_{x}}^{(\infty)} \in \mathcal{K}$ uniquely determines $j^{\infty} \phi_{\left.\right|_{x}}$. The resulting subspace, $\mathcal{B}$, is right-equivariant. To see this note that if $\left(z_{\left.\right|_{x}}^{(\infty)}, j^{\infty} \phi_{\left.\right|_{x}}\right) \in \mathcal{B}$ then, for $\psi \in \mathcal{G}$,

$$
R_{\psi} \cdot\left(\left.z\right|_{x} ^{(\infty)}, j^{\infty} \phi_{\left.\right|_{x}}\right)=\left(j^{\infty} \psi_{\left.\right|_{x}} \cdot z_{\left.\right|_{x}}^{(\infty)},\left.j^{\infty} \varphi_{\left.\right|_{x}} \cdot j^{\infty} \psi^{-1}\right|_{\psi(x)}\right),
$$

and

$$
\left.j^{\infty} \varphi_{\left.\right|_{x}} \cdot \psi^{-1}\right|_{\psi(x)} \cdot\left(j^{\infty} \psi_{\left.\right|_{x}} \cdot z_{\left.\right|_{x}}^{(\infty)}\right)=j^{\infty} \phi_{\left.\right|_{x}} \cdot z_{\left.\right|_{x}}^{(\infty)} \in \mathcal{K},
$$

so $R_{\psi} \cdot\left(\left.z\right|_{x} ^{(\infty)}, j^{\infty} \phi_{\left.\right|_{x}}\right) \in \mathcal{B}$. In practice the cross-section $\mathcal{K}$ is usually built, orderby-order, as the subspace where an increasing number of the jet-coordinates on $J^{\infty}(\mathcal{E}), u_{J}^{\alpha}$, are constant. This will give a decreasing sequence of partial moving frames

$$
\widetilde{\mathcal{G}}_{\infty} \supset \widetilde{\mathcal{B}}_{0} \supset \widetilde{\mathcal{B}}_{1} \supset \widetilde{\mathcal{B}}_{2} \supset \ldots
$$

The partial moving frames are then described by the solutions to equations of the form

$$
U_{J}^{\alpha}=\text { constant }
$$

and we say we have normalized the lifted invariant $U_{J}^{\alpha}$ when $u_{J}^{\alpha}$ is constant on $\mathcal{K}$.

Remark 5.2.5. When restricting a partial moving frame $\widetilde{\mathcal{B}}_{p}$ to a new equation 
$U_{J}^{\alpha}=c$, we solve $U_{J}^{\alpha}=c$ for one of the group parameters. This group parameter then disappears from our parametrization of $\widetilde{\mathcal{B}}_{p}$, and, by a slight abuse of notation, we say that this group parameter has been normalized.

Example 5.2.6. Consider the Lie pseudo-group action of horizontal transformations on sections of $\mathbb{R}^{3} \rightarrow \mathbb{R}^{2}$ obtained by extending the Lie pseudo-group of transformations on $\mathbb{R}^{2}, \mathcal{G}$, with determining equations

$$
X=f(x), \quad Y=f_{x}(x) y+g(x),
$$

to act on a variable $u \in \mathbb{R}$ such that

$$
U=u+\frac{Y_{x}}{X_{x}}
$$

$\mathcal{G}$ has determining equations

$$
X_{y}=0, \quad Y_{y}=X_{x}
$$

We build the cross-section order-by-order, first setting

$$
\mathcal{K}=\{x=y=u=0\} .
$$

A point $\left(z_{x}^{(\infty)}, j^{\infty} \phi_{\left.\right|_{x}}\right)=\left(x, y, u, \ldots, u_{J}^{\alpha}, \ldots, X, Y, \ldots, X_{K}, Y_{K}, \ldots\right)$ is in the corresponding partial moving frame, $\widetilde{\mathcal{B}}_{1} \subset \widetilde{\mathcal{G}}_{\infty}$, if and only if

$$
\begin{aligned}
X & =0, \quad Y=0, \quad U=0 \\
\Leftrightarrow X & =0, \quad Y=0, \quad Y_{y}=-u X_{x} .
\end{aligned}
$$


At the next order, we have lifted invariants

$$
\begin{aligned}
U_{X} & =\frac{u_{x}}{X_{x}}+\frac{Y_{x x} X_{x}-X_{x x} Y_{x}}{X_{x}^{3}}, \\
U_{Y} & =-\frac{Y_{x}}{X_{x}} U_{X}+\frac{u_{y}}{X_{x}}+\frac{Y_{x y}}{X_{x}^{2}} .
\end{aligned}
$$

Notice that we are using the fact that the pseudo-group jets are coming from a pseudo-group with defining equations

$$
X_{y}=0, \quad Y_{x}=X_{x},
$$

and we have replaced all principal derivatives by parametric ones in our formulas for lifted invariants. We can normalize both $U_{X}=U_{Y}=0$ to obtain a partial moving frame $\widetilde{\mathcal{B}}_{2} \subset \widetilde{\mathcal{B}}_{1} \subset \widetilde{\mathcal{G}}$ on which

$$
Y_{x x}=\frac{X_{x x} Y_{x}-X_{x}^{2} u_{x}}{X_{x}}, \quad Y_{y x}=-u_{y} X_{x}
$$

We can continue like this, normalizing $U_{X X}=U_{X Y}=0, U_{Y Y}=1$ and $U_{X X X}=$ $U_{X X Y}=0$ (although it is quite a bit of work). At this point we have actually normalized all pseudo-group parameters of order at most 4. Since each pseudogroup parameter that can normalized can also serve as a leading term in a defining equation $U_{J}^{\alpha}=\bar{u}_{J}^{\alpha}(X)$ we can be sure that all pseudo-group parameters can be normalized; all parameters of order 5 can be normalized at the next order and so on.

Restricting $U_{X Y Y}$ and $U_{Y Y Y}$ to the partial moving $\widetilde{\mathcal{B}}_{4}$ we have constructed so far gives the invariants

$$
U_{X X X} \mapsto \frac{u_{x y y}+u u_{y y y}+2 u_{y} u_{y y}}{u_{y y}^{3 / 2}}, \quad U_{Y Y Y} \mapsto \frac{u_{y y y}}{u_{y y}^{3 / 2}}
$$

This gives the rough idea of how the method of equivariant moving frames proceeds. We will redo this as Example 6.3.1 and shall see how (our modification of) 
Cartan's equivalence method drastically decreases the computational load of the above routine.

Remark 5.2.7. Notice that in 5.12 the jet coordinate $u_{y y}$ must be positive, and so, at some point of our normalization process, we made the decision to restrict our partial moving frame to the subspace of $J^{\infty}(\mathcal{E})$ where $u_{y y}>0$. For jets with $u_{y y}<0$ some of the normalizations made in the above example were not possible, and we could not have constructed this particular partial moving frame. In general, the space $J^{\infty}(\mathcal{E})$ must be partitioned into a collection of subsets

$$
J^{\infty}(\mathcal{E})=\bigcup_{i=1}^{N} \mathcal{S}_{i}
$$

where we obtain a different partial moving frame on each of $\mathcal{S}_{i}$, which we call the domains of definition of the different partial moving frames. In the above example, we have the partition $\mathcal{S}_{1}=\left\{z^{(\infty)}{ }_{\mid x} \mid u_{y y}>0\right\}, \mathcal{S}_{2}=\left\{\left.z^{(\infty)}\right|_{x} \mid u_{y y}=0\right\}$ and $\mathcal{S}_{3}=\left\{z^{(\infty)}{ }_{\mid x} \mid u_{y y}<0\right\}$. The subset $\mathcal{S}_{2}$ is "singular" in the sense that $\mathcal{S}_{2}$ is not locally a $\mathcal{G}$-invariant set. Our combination of Cartan's equivalence method and the equivariant moving frame, built on the results in the next subsection, allows for analysis of these singular jets, cf. Chapter 6 .

\subsection{Sections of $\mathcal{G}_{p}$}

A well known fact is that two submanifolds, of the same dimension, in a Lie group are congruent if and only it there exists a map between them that preserves the pulled-back Maurer-Cartan forms, [18]. In this subsection we shall prove the infinite dimensional analog of this fact.

Consider a Lie pseudo-group, $\mathcal{G}$, of local transformations on the manifold $\mathcal{X}$, determined by formally integrable and regular (and hence locally solvable) differential equations

$$
F\left(x, X^{(q)}\right)=0 .
$$


As noted above, at each order $\mathcal{G}_{q+t}:=\mathcal{G}_{q, t}$ carries a groupoid structure, the groupoid elements of $\mathcal{G}_{p}$ being the $p$-jets $j^{p} \varphi_{\left.\right|_{x}}$ for $\varphi \in \mathcal{G}$. (In the following, it will sometimes be convenient to denote these groupoid elements by lower case Latin letters such as $g$ and $h$.) As mentioned before, the source and target maps endow $\mathcal{G}_{p}$ with a double fibration

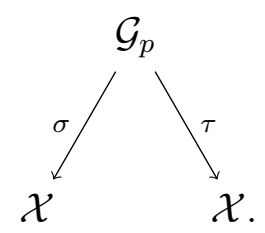

We shall denote the source and target fibers by

$$
\sigma^{-1}(x)=\mathcal{G}_{\left.p\right|_{x}} \quad \text { and } \quad \tau^{-1}(X)=\mathcal{G}_{p}{ }^{X}
$$

Now consider two local sections of $\mathcal{G}_{p} \rightarrow \mathcal{X}, s$ and $\bar{s}$. (Note that $s$ and $\bar{s}$ are not necessarily (indeed, in practice, never will be) the graphs of prolongations of transformations in $\mathcal{G}$, i.e. contact forms do not vanish when restricted to their images.) We want to know whether there exists a local transformation $\varphi \in \mathcal{G}$ such that

$$
R_{\varphi} \cdot s=\bar{s}
$$

Immediate invariants for this problem are the target coordinates of $s$ and $\bar{s}$, since the right-action leaves these invariant. For the time being we consider only sections $s$ and $\bar{s}$ that have constant, and equal, target coordinates,

$$
\tau(s(x))=\tau(\bar{s}(\bar{x}))=X_{0}=\text { constant }
$$

but our results will trivially extend to sections with arbitrary target coordinates (cf. Remark 5.3.6 below). Notice that $s$ and $\bar{s}$ satisfying (5.13) are sections of the bundle $\mathcal{G}_{p}{ }^{X_{0}} \stackrel{\sigma}{\rightarrow} \mathcal{X}$, and so we restrict to these. Obviously, the tangent vectors to the images of $s$ and $\bar{s}$ will then have zero target component. Being tangent to the 
target fibers, we shall call such vectors in $T \mathcal{G}_{p} \tau$-vertical. Tangent vectors that have zero source-components shall be called vertical. Notice that $T\left(\mathcal{G}_{\left.p\right|_{x}}\right)$ is the space of vertical tangent vectors at the source coordinate $x$, while $T\left(G_{p}{ }^{X}\right)$ is the space of $\tau$-vertical vectors at the target coordinate $X$.

As we have seen, a local transformation $\varphi \in \mathcal{G}$ acts on $\mathcal{G}_{p}$ by the left and right actions:

$$
\begin{aligned}
& R_{\varphi} \cdot j^{p} \psi_{\left.\right|_{x}}=\left.j^{p} \psi_{\left.\right|_{x}} \cdot j^{p} \varphi^{-1}\right|_{\varphi(x)}, \quad \text { for all } j^{p} \psi_{\left.\right|_{x}} \text { with source in the domain of } \varphi \\
& L_{\varphi} \cdot j^{p} \psi_{\left.\right|_{x}}=j^{p} \varphi_{\left.\left.\right|_{\tau\left(\left.j^{p} \psi\right|_{x}\right.}\right)} \cdot j^{p} \psi_{\left.\right|_{x}}, \quad \text { for all } j^{p} \psi_{\left.\right|_{x}} \text { with target in the domain of } \varphi
\end{aligned}
$$

On the other hand, for a single groupoid element $g \in \mathcal{G}_{p}$ with $\sigma(g)=x$ we can define the map $R_{g} \cdot h=h \cdot g^{-1}$ for all $h$ with $\sigma(h)=x$ (where $\cdot$ is the groupoid multiplication), i.e. from the source fiber $\mathcal{G}_{\left.p\right|_{\sigma(g)=x}}$ to $\mathcal{G}_{\left.p\right|_{\tau(g)}}$. This map is real-analytic (indeed, it is algebraic) and its derivative is a map between vertical vectors:

$$
R_{g_{*}}: T_{g} \mathcal{G}_{\left.p\right|_{x}} \rightarrow T_{h \cdot g^{-1}} G_{\left.p\right|_{\tau(g)}} .
$$

Similarly, we can define the map $L_{g} \cdot h=g \cdot h$ for all $h \in \mathcal{G}_{p}$ with $\sigma(g)=\tau(h)$. Its differential is a map between $\tau$-vertical vectors:

$$
L_{g_{*}}:\left.T_{g} \mathcal{G}_{p}{ }^{x} \rightarrow T_{g \cdot h} G_{p}\right|^{\tau(g)}
$$

A rather trivial, but important, observation is that the derivative of the right and left actions of a local transformation $\varphi \in \mathcal{G}$ agree with the derivatives of the groupoid actions (5.15) and (5.16) when restricted to vertical, and $\tau$-vertical vectors, respectively.

Lemma 5.3.1. Let $g \in \mathcal{G}_{p}$ be a groupoid element with source $x$ and target $X$. Given a vertical tangent vector $V \in T_{g} \mathcal{G}_{\left.p\right|_{x}}$ and a local transformation $\varphi \in \mathcal{G}$ with domain including $x$, we have

$$
R_{\varphi_{*}} V=R_{\left(\left.j^{p} \varphi\right|_{x}\right)_{*}} V
$$


Where the left hand side is defined by (5.14) and the right hand side by (5.15). Similarly, for a $\tau$-vertical vector $V \in T_{g} \mathcal{G}_{p}{ }^{X}$ we have

$$
L_{\varphi_{*}} V=L_{\left(\left.j^{p} \varphi\right|_{x}\right)_{*}} V
$$

Proof. Let $\Phi(\varepsilon)$ be a path in $\mathcal{G}_{p}$ such that $\left.\frac{d}{d \varepsilon}\right|_{\varepsilon=0} \Phi(\varepsilon)=V$ and such that $\sigma(\Phi(\varepsilon))=$ $x$ for all $\varepsilon$. Then we have

$$
R_{\varphi} \cdot \Phi(\varepsilon)=\Phi(\varepsilon) \cdot j^{p} \varphi^{-1}{ }_{\varphi(x)},
$$

but since each $\Phi(\varepsilon)$ has source $x$, this is trivially equal to

$$
R_{\left.j^{p} \varphi\right|_{x}} \cdot \Phi(\varepsilon),
$$

and the result follows by differentiating (5.17) and (5.18) with respect to $\varepsilon$ and evaluating at $\varepsilon=0$. The second part proceeds similarly.

Turning to the equivalence problem of sections with constant target coordinates, let $s$ and $\bar{s}$ be two sections of $\left.\mathcal{G}_{p}\right|^{X_{0}} \stackrel{\sigma}{\rightarrow} \mathcal{X}$. First assume that a local transformation $\varphi$ exists such that

$$
R_{\varphi} \cdot s=\bar{s} \circ \varphi
$$

Taking the pull-back of a right-invariant Maurer-Cartan form $\mu_{K}^{i},|K|<p$, on $\mathcal{G}_{p}$ on both sides of this equation gives

$$
s^{*} R_{\varphi}^{*} \mu_{K}^{i}=(\bar{s} \circ \varphi)^{*} \mu_{K}^{i} \quad \Longleftrightarrow \quad s^{*} \mu_{K}^{i}=\varphi^{*} \bar{s}^{*} \mu_{K}^{i} .
$$

This means that a necessary condition for there to exist an equivalence map $\varphi$ is that it preserves the pulled-back Maurer-Cartan forms $s^{*} \mu_{K}^{i}$ and $\bar{s}^{*} \mu_{K}^{i}$. We shall prove the converse, i.e. that any local transformation, $f$, of $\mathcal{X}$ that preserves the set of pulled-back forms $s^{*} \mu_{K}^{i}$ and $\bar{s}^{*} \mu_{K}^{i}$ must be a transformation from $\mathcal{G}$ and 
satisfy $R_{f} \cdot s=\bar{s}$. Note that if this result is indeed true and we are given a local transformation $f$ of $\mathcal{X}$ that preserves this collection of one-forms, we have

$$
R_{f(x)} \cdot s(x)=\left.\bar{s}(f(x)) \Longleftrightarrow s(x) \cdot j^{p} f^{-1}\right|_{x}=\bar{s}(f(x))
$$

We can solve for $j^{p} f_{\left.\right|_{x}}$ in this equation to obtain

$$
j^{p} f_{\left.\right|_{x}}=\bar{s}(f(x))^{-1} \cdot s(x) .
$$

Given a local transformation, $f$, of $\mathcal{X}$ that preserves $s^{*} \mu_{K}^{i}$ and $\bar{s}^{*} \mu_{K}^{i}$, our method of proof will be to define a section of $\mathcal{G}_{p}$ by setting

$$
a(x):=\bar{s}(f(x))^{-1} \cdot s(x),
$$

(note that this is indeed a section of $\mathcal{G}_{p}$ ) and proving that all the Maurer-Cartan forms $\mu_{K}^{i},|K|<p$, on $\mathcal{G}_{p}$ vanish when restricted to it. Since the Maurer-Cartan forms are a basis for the contact co-distribution on $\mathcal{G}_{p}$ this means that $j^{p} f_{\left.\right|_{x}}$ must indeed be the prolongation of a local transformation.

Equation (5.19) motivates the definition of the map

$$
\mathbf{m}: U_{\mathbf{m}} \rightarrow \mathcal{G}_{p}, \quad \mathbf{m}(g, h)=g^{-1} \cdot h,
$$

defined on the subset of $\mathcal{G}_{p} \times \mathcal{G}_{p}$ given by

$$
U_{\mathbf{m}}=\left\{(g, h) \in \mathcal{G}_{p} \times \mathcal{G}_{p} \mid \tau(g)=\tau(h)\right\},
$$

i.e. on all pairs $(g, h)$ with a shared target. We need to know how the differential of this map behaves on $\tau$-vertical vectors. Denote the inverse map on $\mathcal{G}_{p}$ by $\mathbf{i}$. This map sends a groupoid element $j^{p} \varphi_{\left.\right|_{x}}$ to $\left.j^{p} \varphi^{-1}\right|_{\varphi(x)}$ and is a diffeomorphism of $\mathcal{G}_{p}$. Notice that the differential $\mathbf{i}_{*}$ maps vertical tangent vectors to $\tau$-vertical ones, and vice versa. 
Lemma 5.3.2. Let $V \in T_{g} \mathcal{G}_{p}{ }^{{ }^{\tau(g)}}$ and $W \in T_{h} \mathcal{G}_{p}{ }^{\tau(h)}$ be $\tau$-vertical vectors on $\mathcal{G}_{p}$ with $(g, h) \in U_{\mathbf{m}}$. Then

$$
\mathbf{m}_{*}(V, W)=R_{h_{*}^{-1}} \mathbf{i}_{*} V+L_{g_{*}^{-1}} W .
$$

Proof. The $\tau$-vertical tangent vectors at each point of $\mathcal{G}_{p}$ form a vector space, and there must be some linear maps $A: T_{g} \mathcal{G}_{p}{ }^{\tau(g)} \rightarrow T_{g^{-1 . h}} \mathcal{G}_{p}$ and $B: T_{h} \mathcal{G}_{p}{ }^{\tau(h)} \rightarrow$ $T_{g^{-1} . h} \mathcal{G}_{p}$ such that

$$
\mathbf{m}_{*}(V, W)=A V+B W .
$$

Now consider $\mathbf{m}_{*}(V, 0)$, where $V \in T_{g} \mathcal{G}_{p}{ }^{\tau(g)}$ and $0 \in T_{h} \mathcal{G}_{p}{ }^{\tau(h)}$, and let $\Phi(\varepsilon)$ be a path in $\mathcal{G}_{p}$ with $\tau(\Phi(\varepsilon))=\tau(g)=\tau(h)$ constant and with $\left.\frac{d}{d \varepsilon}\right|_{\varepsilon=0} \Phi(\varepsilon)=V$. Then

$$
\begin{aligned}
\mathbf{m}_{*}(V, 0) & =\left.\frac{d}{d \varepsilon}\right|_{\varepsilon=0} \mathbf{m}(\Phi(\varepsilon), h)=\left.\frac{d}{d \varepsilon}\right|_{\varepsilon=0}\left(\Phi(\varepsilon)^{-1} \cdot h\right) \\
& =\left.\frac{d}{d \varepsilon}\right|_{\varepsilon=0}\left(R_{h^{-1}} \cdot \mathbf{i}(\Phi(\varepsilon))\right)=R_{h_{*}^{-1}} \mathbf{i}_{*} V .
\end{aligned}
$$

This means that $A=R_{h_{*}^{-1}} \mathbf{i}_{*}$. To find $B$, we choose a path $\Phi(\varepsilon)$ with constant target $\tau(\Phi(\varepsilon))=\tau(g)=\tau(h)$ such that $\left.\frac{d}{d \varepsilon}\right|_{\varepsilon=0} \Phi(\varepsilon)=W$ and compute (here $\left.0 \in T_{g} \mathcal{G}_{p}{ }^{\tau(g)}\right)$

$$
\begin{aligned}
\mathbf{m}_{*}(0, W) & =\left.\frac{d}{d \varepsilon}\right|_{\varepsilon=0} \mathbf{m}(g, \Phi(\varepsilon))=\left.\frac{d}{d \varepsilon}\right|_{\varepsilon=0}\left(g^{-1} \cdot \Phi(\varepsilon)\right) \\
& =\left.\frac{d}{d \varepsilon}\right|_{\varepsilon=0}\left(L_{g^{-1}} \cdot \Phi(\varepsilon)\right)=L_{g_{*}^{-1}} W .
\end{aligned}
$$

This proves the lemma.

We need one more preliminary observation before proving our main theorem. We denote the identity section of $\mathcal{G}_{p}$ by $\mathbb{1}$.

Lemma 5.3.3. For a $\tau$-vertical $V \in T_{g} \mathcal{G}_{p}{ }^{\tau(g)}$, we have

$$
\mathbf{m}_{*}(V, V)=R_{g_{*}^{-1}} \mathbf{i}_{*} V+L_{g_{*}^{-1}} V=\mathbb{1}_{*} \sigma_{*} V .
$$


Proof. Notice that $\mathbf{m}(g, g)=g^{-1} \cdot g=\mathbb{1}(\sigma(g))$, and so, given a path $\Phi(\varepsilon)$ with $\left.\frac{d}{d \varepsilon}\right|_{\varepsilon=0} \Phi(\varepsilon)=V$ we have

$$
\mathbf{m}_{*}(V, V)=\left.\frac{d}{d \varepsilon}\right|_{\varepsilon=0} \Phi(\varepsilon)^{-1} \cdot \Phi(\varepsilon)=\left.\frac{d}{d \varepsilon}\right|_{\varepsilon=0} \mathbb{1}\left(\sigma(\Phi(\varepsilon))=\mathbb{1}_{*} \sigma_{*} V .\right.
$$

But by Lemma 5.3.2 we also have

$$
\mathbf{m}_{*}(V, V)=R_{g_{*}^{-1}} \mathbf{i}_{*} V+L_{g_{*}^{-1}} V
$$

proving the lemma.

Theorem 5.3.4. Let $s$ and $\bar{s}$ be two sections of $\mathcal{G}_{p}{ }^{X_{0}} \stackrel{\sigma}{\rightarrow} \mathcal{X}$ and let $f$ be a local transformation such that

$$
f^{*} \bar{s}^{*} \mu_{K}^{i}=s^{*} \mu_{K}^{i}, \quad|K|<p .
$$

Then $f \in \mathcal{G}$ and $R_{f} \cdot s=\bar{s}$.

Proof. Consider the section of $\mathcal{G}_{p}$ given by

$$
a(x)=\bar{s}(f(x))^{-1} \cdot s(x)=\mathbf{m} \circ(\bar{s}(f(x)), s(x)) .
$$

Notice that this section agrees with $j^{0} f(x)$, i.e. the zero-jet of $f$. We shall prove that $a$ is the prolongation of a local transformation by showing $a^{*} \mu_{K}^{i}=0$, for all $|K|<p$. Since $a$ agrees with $f$ at the zero order, we must have $a(x)=j^{p} f_{\left.\right|_{x}}$ and since (by definition of a) $R_{a(x)} \cdot s(x)=\bar{s}\left(f(x)\right.$ ), we have $R_{f} \cdot s=\bar{s}$.

Let $v \in T_{x} \mathcal{X}$ be a tangent vector to $\mathcal{X}$ in the domain of $s$. We compute, using Lemma 5.3.2,

$$
a^{*} \mu_{K}^{i}(v)=\mathbf{m}^{*} \mu_{K}^{i}\left((\bar{s} \circ f)_{*} v, s_{*} v\right)=\mu_{K}^{i}\left(R_{s(x)_{*}^{-1}} \mathbf{i}_{*}(\bar{s} \circ f)_{*} v+L_{\bar{s}(f(x))_{*}^{-1}} s_{*} v\right) .
$$

Consider $\mu_{K}^{i}\left(L_{\bar{s}(f(x))_{*}^{-1}} s_{*} v\right)$. Since the determining equations for $\mathcal{G}_{p}$ are locally solvable, we can choose a local solution $\psi \in \mathcal{G}$ such that $j^{p} \psi_{\left.\right|_{X_{0}}}=\bar{s}(f(x))^{-1}$ and 
because of Lemma 5.3.1 we have

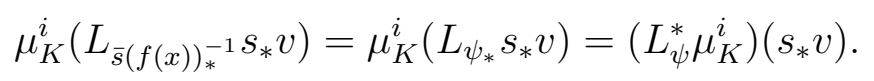

But $L_{\psi}^{*} \mu_{K}^{i}$ is a right-invariant contact form (of order $|K|$ ) since the left and right actions commute and $L_{\psi}$ is a contact transformation on $\mathcal{G}_{p}$. Since the $\mu_{K}^{i}$ are a basis for right-invariant contact forms on $\mathcal{G}_{p}$ and $s^{*} \mu_{K}^{i}=(\bar{s} \circ f)^{*} \mu_{K}^{i}$ we also have $s^{*}\left(L_{\psi}^{*} \mu_{K}^{i}\right)=(\bar{s} \circ f)^{*}\left(L_{\psi}^{*} \mu_{K}^{i}\right)$. Continuing (5.21), and applying Lemma 5.3.1 again, we get

$$
\left(L_{\psi}^{*} \mu_{K}^{i}\right)\left(s_{*} v\right)=\left(L_{\psi}^{*} \mu_{K}^{i}\right)\left((\bar{s} \circ f)_{*} v\right)=\mu_{K}^{i}\left(L_{\psi_{*}}(\bar{s} \circ f)_{*} v\right)=\mu_{K}^{i}\left(L_{\bar{s}(f(x))_{*}^{-1}}(\bar{s} \circ f)_{*} v\right) .
$$

The last expression, according to Lemma 5.3.3, is equal to

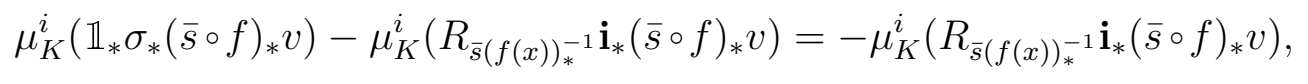

since $\mathbb{1}^{*} \mu_{K}^{i}=0$, as $\mathbb{1}$ annihilates contact forms under pull-back. Plugging this into $(5.20)$ we arrive at

$$
a^{*} \mu_{K}^{i}(v)=\mu_{K}^{i}\left(R_{s(x)_{*}^{-1}} \mathbf{i}_{*}(\bar{s} \circ f)_{*} v\right)-\mu_{K}^{i}\left(R_{\left(\bar{s}(f(x))_{*}^{-1}\right.} \mathbf{i}_{*}(\bar{s} \circ f)_{*} v\right) .
$$

Now, as before, choose two local transformations $\varphi, \psi \in \mathcal{G}$ such that

$$
j^{p} \varphi_{\left.\right|_{X_{0}}}=s(x)^{-1}, \quad j^{p} \psi_{\left.\right|_{X_{0}}}=\bar{s}(f(x))^{-1} .
$$

According to Lemma 5.3.1, we can write (5.23) as

$$
\begin{aligned}
a^{*} \mu_{K}^{i}(v) & =\mu_{K}^{i}\left(R_{\varphi_{*}} \mathbf{i}_{*}(\bar{s} \circ f)_{*} v\right)-\mu_{K}^{i}\left(R_{\psi_{*}} \mathbf{i}_{*}(\bar{s} \circ f)_{*} v\right) \\
& =\mu_{K}^{i}\left(\mathbf{i}_{*}(\bar{s} \circ f)_{*} v\right)-\mu_{K}^{i}\left(\mathbf{i}_{*}(\bar{s} \circ f)_{*} v\right) \\
& =0
\end{aligned}
$$

since $R_{\varphi}^{*} \mu_{K}^{i}=R_{\psi}^{*} \mu_{K}^{i}=\mu_{K}^{i}$. 
We easily obtain the following useful corollary.

Corollary 5.3.5. Let $\mathcal{G}_{p}$ be formally integrable, let $X \in \mathcal{X}$ and let $\Lambda: \mathcal{G}_{p}{ }^{X_{0}} \rightarrow$ $\mathcal{G}_{p}{ }^{X_{0}}$ be a map on a $\tau$-vertical fiber. Then $\Lambda$ preserves the Maurer-Cartan forms $\mu_{K}^{i}$ (restricted to $\mathcal{G}_{p}$ ), $|K|<p$, if and only if $\Lambda=R_{\varphi}$ for some $\varphi \in \mathcal{G}$.

Proof. If $\Lambda=R_{\varphi}$ for $\varphi \in \mathcal{G}$ it obviously preserves the $\mu_{K}^{i}$. Conversely, assume $\Lambda^{*} \mu_{K}^{i}=\mu_{K}^{i}$ for all $|K|<p$. Then $\Lambda$ is a diffeomorphism and, since $\mu^{i}=-\omega^{i}$ on $\mathcal{G}_{p}{ }^{X_{0}}$, we have $\Lambda^{*} \omega^{i}=\omega^{i}$. Let $s$ be any section of $\mathcal{G}_{p}{ }^{X_{0}} \stackrel{\sigma}{\rightarrow} \mathcal{X}$ and denote by $\bar{s}$ the image of $\Lambda(s)$. Then $\bar{s}$ is also a section of $\mathcal{G}_{p}{ }^{{ }^{X}}$ since

$$
\Lambda^{*}\left(\left.\omega^{1} \wedge \ldots \omega^{n}\right|_{\bar{s}}\right)=\left.\omega^{1} \wedge \cdots \wedge \omega^{n}\right|_{s} \neq 0
$$

Then, according to Theorem 5.3.4. $\Lambda$ agrees with $R_{\varphi}$, for some $\varphi \in \mathcal{G}$, when restricted to $s$ and since the section $s$ was arbitrary, we also must have $\Lambda=R_{p}$.

Remark 5.3.6. There is a slight generalization of Lemma 5.3 .2 available that will provide the solution to the equivalence of general sections of $\mathcal{G}_{p}$. To describe it we define the operator $t$ taking tangent vectors in $T \mathcal{G}_{p}$ to $\tau$-vertical ones via

$$
t(V)=V-\tau_{*} V
$$

Note that any two tangent vectors $V, W$ to $U_{\mathbf{m}}$ satisfy $\tau(V)=\tau(W)$.

Lemma 5.3.7. Let $V, W \in T U_{\mathbf{m}}$ be tangent vectors at $g$ and $h$, respectively. Then

$$
\mathbf{m}_{*}(V, W)=\mathbf{m}_{*}(t(V), t(W))=R_{h_{*}^{-1}} \mathbf{i}_{*} t(V)+L_{g_{*}^{-1}} t(W) .
$$

The proof is identical to the proof of Lemma 5.3.2. Now, when two sections, $s$ and $\bar{s}$, of $\mathcal{G}_{p}$ do not have fixed target coordinates, $\tau(s(x))=\tau(\bar{s}(\bar{x}))=X_{0}$, any equivalence map $R_{\varphi}$ between them must preserve the invariants

$$
I(x):=\tau(s(x)) \quad \text { and } \quad \bar{I}(\bar{x}):=\tau(\bar{s}(\bar{x})) .
$$


Conversely, given a local map $f: \mathcal{X} \rightarrow \mathcal{X}$ that preserves the pulled-back MaurerCartan coframes

$$
f^{*} \bar{s}^{*} \mu_{K}^{i}=s^{*} \mu_{K}^{i}
$$

and the invariants $I$ and $\bar{I}$,

$$
\bar{I}(f(x))=I(x)
$$

our proof of Theorem 5.3.4 goes through essentially unchanged; we just have to replace $(\bar{s} \circ f)_{*} v$ and $s_{*} v$ by $t\left((\bar{s} \circ f)_{*} v\right)$ and $t\left(s_{*} v\right)$ after the last equality in (5.20) and throughout.

Remark 5.3.8. We mention that by the recurrence formula, on each $\tau$-vertical fiber $\mathcal{G}^{\left.\right|^{X_{0}}}$, we have

$$
\mu^{i}=-\omega^{i},
$$

and in the forthcoming application of the above results we shall usually work with the $\omega^{i}$.

\section{$5.4 G$-structures and partial moving frames}

Let a Lie pseudo-group $\mathcal{G}$ of local diffeomorphisms on $\mathcal{X}$ be determined by a set of first order equations (this is purely a simplifying, not a necessary, assumption). Assume the right-action of $\mathcal{G}$ is extended to a space $\mathcal{U}$ such that the action in the variables $u \in \mathcal{U}$ depends only on the first order jets of transformations from $\mathcal{G}$. As before, we ask when two sections in $\mathcal{E}=\mathcal{X} \times \mathcal{U} \rightarrow \mathcal{X}$ are congruent under a transformation from $\mathcal{G}$. It will be convenient to have a running example during this section, but this next example also introduces our key idea.

Example 5.4.1. Let $\mathcal{G}$ be the Lie pseudo-group of contact-transformations in the variables $z=\left(x, u, u_{x}\right)=(x, u, p)$ determined by the differential equations

$$
X_{u}=X_{p}=U_{p}=0, \quad P=\frac{U_{x}+p U_{u}}{X_{x}}, \quad P_{p}=\frac{U_{u}}{X_{x}},
$$


extended to act on sections in $\mathbb{R}^{3} \times \mathbb{R} \rightarrow \mathbb{R}^{3}$ by letting $\mathcal{G}_{1}$ act on the fiber coordinate by

$$
q \mapsto Q\left(j^{1} \varphi_{z}, q\right)=\frac{P_{x}+p P_{u}+q P_{p}}{X_{x}}
$$

Now, for the moment assume (which is actually the case) that the determining equations (5.24) for $\mathcal{G}$ are formally integrable and thus locally solvable. Then Theorem 5.3.4 says that two sections, $s$ and $\bar{s}$, with fixed target coordinates, say $Z=(X, U, P)=0$, of $\mathcal{G}_{1}$ are congruent under the right action of a $\psi \in \mathcal{G}$ if and only if $\psi$ preserves the pull-backs of $\omega^{x}, \omega^{u}$ and $\omega^{p}$ (Recall Remark 5.3.8). In all applications we consider it will be sufficient to work with sections with fixed target coordinates, not needing the more general result of Remark 5.3.6 and so we restrict this discussion to this caes. This is just for simplification; all our results extend trivially to the more general non-transitive case.

But what has to happen to guarantee that a local transformation $\psi \in \mathcal{G}$ such that $R_{\psi} \cdot s=\bar{s}$ also maps a local section of $\mathcal{E}$ to another such section? Letting these sections be the graphs of two locally defined functions $q_{0}, \bar{q}_{0}: \mathcal{X} \rightarrow \mathcal{U}$, we claim that this is the case if and only if $R_{\psi}$ also preserves the lifted invariant $Q\left(j^{1} \varphi_{\left.\right|_{z}}, q\right)$ restricted to $\left(\left.s\right|_{z}, q_{0}(z)\right)$ and $\left(\bar{s}_{\left.\right|_{\bar{z}}}, \bar{q}_{0}(\bar{z})\right)$. That is

$$
Q\left(s_{\left.\right|_{z}}, q_{0}(z)\right)=Q\left(\bar{s}_{\mid \psi(z)}, \bar{q}_{0}(\psi(z))\right)
$$

Letting $\psi$ satisfy $R_{\psi} \cdot s=\bar{s}$ and (5.25) we obtain:

$$
\begin{aligned}
& Q\left(s_{\left.\right|_{z}} \cdot j^{1} \psi_{\left.\right|_{\psi(z)}}^{-1}, Q\left(j^{1} \psi_{\left.\right|_{z}}, q_{0}(z)\right)\right) & & \\
= & Q\left(s_{\left.\right|_{z}}, q_{0}(z)\right) & & \text { by right invariance of } Q \\
= & Q\left(s_{\left.\right|_{z}} \cdot j^{1} \psi_{\left.\right|_{\psi(z)}}^{-1}, \bar{q}_{0}(\psi(z))\right) & & \text { by } 5.25 .
\end{aligned}
$$

Comparing the first and last equations we can see that, if $Q(g, q)$ is full rank in the $q$ variable, we have

$$
Q\left(j^{1} \psi_{\left.\right|_{z}}, q(z)\right)=\bar{q}(\psi(z)) .
$$


But this means that $\psi$ also maps $q_{0}$ to $\bar{q}_{0}$.

The computations at the end of the last example easily generalize to prove the following.

Theorem 5.4.2. Let $\mathcal{G}$ be a horizontal action on a trivial bundle $\mathcal{E}=\mathcal{X} \times \mathcal{U} \rightarrow \mathcal{X}$ whose $q^{\text {th }}$ order defining equations are formally integrable. Assume also that the zero order lifted invariants $U\left(j^{1} \varphi_{\left.\right|_{x}}, u\right)=\lambda(u)$ are full rank in the $u$ variables. Given two local functions, $u, \bar{u}: \mathcal{X} \rightarrow \mathcal{U}$, there is a map $\varphi \in \mathcal{G}$ such that $\varphi \cdot u(x)=$ $\bar{u}(\varphi(x))$ if and only if there are two sections of some target fiber $\mathcal{G}_{q}{ }^{X_{0}} \stackrel{\sigma}{\rightarrow} \mathcal{X}$, $s$ and $\bar{s}$, such that $R_{\varphi}$ maps $s$ to $\bar{s}$ and such that $R_{\varphi}$ preserves the zero order lifted invariants $U=\lambda(u)$ and $\bar{U}=\lambda(\bar{u})$ restricted to $\left(s_{\left.\right|_{x}}, u(x)\right)$ and $\left(\bar{s}_{\mid \bar{x}}, \bar{u}(\bar{x})\right)$ respectively.

Continuing Example 5.4.1, we next introduce Élie Cartan's fundamental idea underlying his equivalence method.

Example 5.4.3. Continuing Example 5.4.1, we are given two local functions $q_{0}$ and $\bar{q}_{0}$ and wish to characterize all equivalence maps, $\varphi \in \mathcal{G}$, between them. We can do this, according to Theorem 5.4 .2 , by constructing two sections, $s$ and $\bar{s}$, of $\mathcal{G}_{1}$ and requiring that $\varphi$, or, rather $R_{\varphi}$, maps $s$ to $\bar{s}$ all the while preserving the lifted invariant

$$
Q=\frac{P_{x}+p P_{u}+q P_{p}}{X_{x}}
$$

restricted to $\left(s, q_{0}\right)$ and $\left(\bar{s}, \bar{q}_{0}\right)$. But, and here is the key observation (of Élie Cartan), since we get to choose the sections $s$ and $\bar{s}$ ourselves, we might as well choose them to lie in the subset of $\mathcal{G}_{1}$ where $Q \equiv 0$. Then, any map between $s$ and $\bar{s}$ automatically preserves the restricted lifted invariant $Q$. Taking a closer look at this, we, for example, require that the components of $s$ satisfy

$$
Q=\frac{P_{x}+p P_{u}+q_{0}(z) P_{p}}{X_{x}}=0 \quad \Leftrightarrow \quad P_{x}=-p P_{u}-q_{0}(z) P_{p}
$$

and similarly for $\bar{s}$ and $\bar{q}_{0}$. Referring back to our fundamental Theorem 5.3.4, the equivalence problem has now been reduced to finding a local transformation 
$f$ of $\mathcal{X}$, and two sections, $s$ and $\bar{s}$, of the partial moving frame determined by $X=U=P=Q=0$, such that

$$
f^{*} \bar{s}^{*} \omega^{i}=s^{*} \omega^{i}
$$

Since we can restrict to sections of a partial moving frame, the recurrence formula will hold all the relevant information of the structure of the Maurer-Cartan forms thereon. For example, by the recurrence formula, we have

$$
0=d Q=\mu_{x}^{p}+Q_{X} \omega^{x}+Q_{U} \omega^{u}+Q_{P} \omega^{p},
$$

on $Q=0$, where, from now on, we implicitly assume that the lifted invariants are evaluated at a specific section of $\mathcal{E} \rightarrow \mathcal{X}$, like in 5.26 .

Generalizing the above example, we have the following corollary to Theorem 5.4 .2

Corollary 5.4.4. Let the set-up be the same as in Theorem 5.4.2 and let $\mathcal{B}_{p}$, for $p \geq q$, be a $p^{\text {th }}$ order partial moving frame. Then the graphs of two local functions, $u$ and $\bar{u}$, are congruent under $\mathcal{G}$ if and only if there are two sections, $s$ and $\bar{s}$ of $\mathcal{B}_{p} \stackrel{\sigma}{\rightarrow} \mathcal{X}$, such that, when they are restricted to $j^{p} u$ and $j^{p} \bar{u}$, respectively, they are congruent under the right action of $\mathcal{G}$.

Remark 5.4.5. When we normalize a lifted invariant the recurrence formula (4.15) tells us what effect that has on the structure of the Maurer-Cartan forms on the partial moving frame. We have,

$$
0=d U^{\alpha}=U_{i}^{\alpha} \omega^{i}+\sum_{|K| \leq 1} \lambda\left(\left.\frac{\partial U^{\alpha}}{\partial X_{K}^{i}}\right|_{\mathbb{1}}\right) \mu_{K}^{i}+\text { contact forms on } J^{\infty}(\mathcal{E}) .
$$

When we restrict to the graph of a specific function, $u$, the contact forms on $J^{\infty}(\mathcal{E})$ vanish. Since an equivalence map between the graphs of $u$ and $\bar{u}$ must preserve all the Maurer-Cartan forms restricted to the partial moving frame, we 
can see that such an equivalence map must also preserve the lifted invariants $U_{i}^{\alpha}$. We may normalize these to produce a new partial moving frame whose sections, restricted to $u$ and $\bar{u}$, are equivalent if and only if $u$ and $\bar{u}$ are. The next subsection continues this discussion.

Cartan's equivalence method, and, indeed, most past approaches to Lie pseudogroups have been based on the concept of a $G$-structure.

Definition 5.4.6. A $G$-structure for an equivalence problem of sections of $\mathcal{E} \rightarrow \mathcal{X}$ under the extended action of a Lie pseudo-group $\mathcal{G} \rightarrow \mathcal{X}$ is a pair $\{G, \boldsymbol{\eta}\}$ where $G$ is a subgroup of the general linear group $G L(n)$ and $\boldsymbol{\eta}=\left\{\eta^{1}, \ldots, \eta^{n}\right\}$ is a coframe of $\mathcal{X}$ (that depends on jets of sections of $\mathcal{E}$ ), that satisfies the following. A transformation $\varphi \in \mathcal{G}$ maps the graph of $u$ to the graph of $\bar{u}$ if and only if $\boldsymbol{\eta}$, restricted to the two graphs, is preserved, up to an element of $G \subset G L(n)$, under the pull-back of $\varphi$. That is

$$
\varphi^{*}\left[\begin{array}{c}
\eta^{1}{ }_{\mid \bar{u}} \\
\vdots \\
\eta^{n}{ }_{{ }_{u}}
\end{array}\right]=g \cdot\left[\begin{array}{c}
\eta^{1}{ }_{{ }_{u}} \\
\vdots \\
\eta^{n}{ }_{{ }_{u}}
\end{array}\right]
$$

for an element $g \in G$.

Remark 5.4.7. We note that the condition (5.27) is equivalent to there existing two locally defined functions, $s$ and $\bar{s}$ from $\mathcal{X}$ to $G \subset G L(n)$ such that

$$
\varphi^{*}\left(\bar{s}(\bar{x}) \cdot\left[\begin{array}{c}
\eta^{1}{ }_{{ }_{\bar{u}}} \\
\vdots \\
\eta^{n}{ }_{{ }_{\bar{u}}}
\end{array}\right]\right)=s(x) \cdot\left[\begin{array}{c}
\eta^{1}{ }_{{ }_{u}} \\
\vdots \\
\eta^{n}{ }_{{ }_{u}}
\end{array}\right] .
$$

Given such $s$ and $\bar{s}$ we can let $g=\bar{s}(\varphi(x))^{-1} \cdot s(x)$ to recover (5.27). The $G$ structure approach to equivalence problems then boils down to finding two equivalent sections, $s$ and $\bar{s}$ of the trivial bundle $\mathcal{X} \times G \rightarrow \mathcal{X}$, the first restricted to $u$ and the second to $\bar{u}$, just like in our partial moving frame approach. 
Restricting the Maurer-Cartan forms $\mu_{K}^{i},|K|<p$, on $\mathcal{G}_{p}$ to a partial moving frame encodes the equivalence problem for sections as that between collections of one-forms, pulled-back from the partial moving frame, by Corollary 5.4.4. In favorable cases a $G$-structure arises from this restriction. Let us look at a few wellknown examples of where $G$-structures arise in this manner. (But, as emphasized by Cartan himself, [7, his equivalence algorithm need not be restricted to $G$ structures. Indeed, the hybrid equivalence method presented in Chapter 5 will require no such restrictions.)

Example 5.4.8. Consider the point transformation counterpart to our running example. That is, the Lie pseudo-group, $\mathcal{G}$, with defining equations $X_{p}=U_{p}=0$ and

$$
P=\frac{U_{x}+p U_{u}}{X_{x}+p X_{u}}
$$

acting on $q$ by

$$
q \mapsto \frac{P_{x}+p P_{u}+q P_{q}}{X_{x}+p X_{u}} .
$$

Normalizing the lifted invariants $P$ and $Q$ to zero, we get $U_{x}=-p U_{u}$ and $P_{x}=$ $-q P_{p}-p P_{u}$. Notice that differentiating the defining equation (5.28) with respect to $p$ gives the first order integrability condition

$$
p X_{u}+X_{x}=\frac{U_{u}-P X_{u}}{P_{p}},
$$

which becomes $X_{x}+p X_{u}=\frac{U_{u}}{P_{p}}$ after setting $P=0$. Including this integrability condition makes $\mathcal{G}_{1}$ formally integrable. We have, on the partial moving frame $\mathcal{B}_{1}=\{X=U=P=Q=0\}$ that

$$
\begin{aligned}
& \omega^{x}=X_{x} d x+X_{u} d u=\left(p X_{u}+X_{x}\right) d x+X_{u}(d u-p d x)=\frac{U_{u}}{P_{p}} d x+X_{u}(d u-p d x), \\
& \omega^{u}=-p U_{u} d x+U_{u} d u=U_{u}(d u-p d x), \\
& \omega^{p}=\left(-q P_{p}-p P_{u}\right) d x+P_{u} d u+P_{p} d p=P_{u}(d u-p d x)+P_{p}(d p-q d x) .
\end{aligned}
$$

If we further restrict to a specific function $q=f(x, u, p)$, we obtain the $G$ structure 
of Cartan for this problem, see [36]: set $\eta^{1}=d x, \eta^{2}=d u-p d x$ and $\eta^{3}=d p-f d x$ as a base coframe on $J^{1}$ and note that the lifted horizontal coframe can be written as

$$
\left[\begin{array}{c}
\omega^{x} \\
\omega^{u} \\
\omega^{p}
\end{array}\right]=\left[\begin{array}{ccc}
\frac{U_{u}}{P_{p}} & X_{u} & 0 \\
0 & U_{u} & 0 \\
0 & P_{u} & P_{p}
\end{array}\right]\left[\begin{array}{c}
\eta^{1} \\
\eta^{2} \\
\eta^{3}
\end{array}\right]=\left[\begin{array}{ccc}
\frac{a_{1}}{a_{2}} & a_{3} & 0 \\
0 & a_{1} & 0 \\
0 & a_{4} & a_{2}
\end{array}\right]\left[\begin{array}{c}
\eta^{1} \\
\eta^{2} \\
\eta^{3}
\end{array}\right] .
$$

By Theorem 5.4.2 this is indeed a $G$-structure with $G \subset G L(3)$ being the subgroup of all matrices of the form

$$
\left[\begin{array}{ccc}
\frac{a_{1}}{a_{2}} & a_{3} & 0 \\
0 & a_{1} & 0 \\
0 & a_{4} & a_{2}
\end{array}\right], \quad a_{1} a_{2} \neq 0
$$

Example 5.4.9. Understandably, the pseudo-group of contact transformations, $\mathcal{G}$, is prominent in equivalence problems for differential equations. One facet of which is the equivalence problem of Lagrangians $\int L(x, u, p) d x$ and $\int \bar{L}(X, U, P) d X$. An element $\varphi \in \mathcal{G}$ transforms Lagrangians according to

$$
\int \bar{L}(X, U, P) d X \mapsto \int \bar{L}(X, U, P) \cdot\left(X_{x}+p X_{u}\right) d x
$$

and we see that the equivalence problem can be written as the PDE

$$
\bar{L}(X, U, P) \cdot\left(X_{x}+p X_{u}\right)=L(x, u, p) \Leftrightarrow \bar{L}(X, U, P)=\frac{L(x, u, p)}{X_{x}+p X_{u}} .
$$

If we extend $\mathcal{G}$ to act on a new variable $L$ by

$$
L \mapsto \frac{L}{X_{x}+p X_{u}},
$$

then the equivalence problem of Lagrangians is equivalent to that of sections in this extended space. Normalizing the lifted invariant $\frac{L}{X_{x}+p X_{u}}$ to 1 , and, as before, $P=\frac{U_{x}+p U_{u}}{X_{x}+p X_{u}}$ to 0 , we have $X_{x}+p X_{u}=L, U_{x}=-p U_{u}$ and recalling the 
integrability condition $X_{x}+p X_{u}=\frac{U_{u}}{P_{p}}$ we have

$$
\begin{aligned}
& \omega^{x}=X_{x} d x+X_{u} d u=\left(p X_{u}+X_{x}\right) d x+X_{u}(d u-p d x)=L d x+X_{u}(d u-p d x), \\
& \omega^{u}=-p U_{u} d x+U_{u} d u=U_{u}(d u-p d x), \\
& \omega^{p}=P_{x} d x+P_{u} d u+P_{p} d p=\frac{P_{x}+p P_{u}}{L} L d x+P_{u}(d u-p d x)+P_{p} d p .
\end{aligned}
$$

Letting $a_{1}=X_{u}, a_{2}=P_{p}, a_{3}=P_{u}$ and $a_{4}=\frac{P_{x}+p P_{u}}{L}$ we recover a $G$-structure for this problem, with the base coframe $\left\{\eta^{1}, \eta^{2}, \eta^{3}\right\}=\{L d x, L(d u-p d x), d p\}$ :

$$
\left[\begin{array}{l}
\omega^{x} \\
\omega^{u} \\
\omega^{p}
\end{array}\right]=\left[\begin{array}{ccc}
1 & a_{1} & 0 \\
0 & a_{2} & 0 \\
a_{4} & a_{3} & a_{2}
\end{array}\right]\left[\begin{array}{l}
\eta^{1} \\
\eta^{2} \\
\eta^{3}
\end{array}\right] .
$$

Example 5.4.10. Sometimes a little work is required for the best possible formulation of an equivalence problem. Expanding on Example 5.4.9, we can consider the divergence equivalence of first order Lagrangians under point transformations (as in the previous example). It is well known that two Lagrangians produce the same Euler-Lagrange equations if they differ by a total derivative $D_{x} A(x, u)$, so the equivalence problem

$$
\bar{L}(X, U, P) \cdot\left(X_{x}+p X_{u}\right)=L(x, u, p)+D_{x} A
$$

where $A(x, u)$ is some (real-analytic) function, is of interest. For our first order Lagrangian, $L$, the Euler-Lagrange expression has the form

$$
E(L):=L_{u}-L_{p x}-p L_{p u}-q L_{p p}
$$


where $q$ is the second derivative of $u$. Under a contact transformation, the EulerLagrange equations transform according to

$$
E(L) \mapsto \frac{E(L)}{U_{u} X_{x}-U_{x} X_{u}}=\frac{E(L)}{P_{p} \cdot\left(X_{x}+p X_{u}\right)^{2}},
$$

where the second equality follows from $P=\frac{U_{x}+p U_{u}}{X_{x}+p X_{u}}$. It will be convenient to define the truncated Euler-Lagrange expression $\widetilde{E}(L):=L_{u}-L_{p x}-p L_{p u}$. Let us also denote the source coordinates $(x, u, p)$ by $t$ and the target coordinates $(X, U, P)$ by $T$. If two Lagrangians, $L$ and $\bar{L}$, are divergence equivalent, then their Euler-Lagrange equations are equivalent. This means that we must have

$$
E(\bar{L})(T)=\frac{E(L)(t)}{P_{p} \cdot\left(X_{x}+p X_{u}\right)^{2}}
$$

Writing this out, and remembering that $q$ transforms according to

$$
q \mapsto Q=\frac{P_{x}+p P_{u}+q P_{p}}{X_{x}+p X_{u}}
$$

we have

$$
\begin{aligned}
& \bar{L}_{u}(T)-\bar{L}_{p x}(T)-P \bar{L}_{p u}(T)-\left(\frac{P_{x}+p P_{u}+q P_{p}}{X_{x}+p X_{u}}\right) \bar{L}_{p p}(T) \\
& =\frac{L_{u}(t)-L_{p x}(t)-p L_{p u}(t)-q L_{p p}(t)}{P_{p} \cdot\left(X_{x}+p X_{u}\right)^{2}} .
\end{aligned}
$$

Viewing this as a first degree polynomial in $q$, and comparing coefficients we obtain

$$
\bar{L}_{u}(T)-\bar{L}_{p x}(T)-P \bar{L}_{p u}(T)-\left(\frac{P_{x}+p P_{u}}{X_{x}+p X_{u}}\right) \bar{L}_{p p}(T)=\frac{L_{u}(t)-L_{p x}(t)-p L_{p u}(t)}{P_{p} \cdot\left(X_{x}+p X_{u}\right)^{2}}
$$

and

$$
\frac{P_{p}}{X_{x}+p X_{u}} \bar{L}_{p p}(T)=\frac{L_{p p}(t)}{P_{p} \cdot\left(X_{x}+p X_{u}\right)^{2}}
$$

Solving for $L_{p p}(T)$ in the second equation, plugging the result into the first and 
moving some terms around gives

$$
\bar{L}_{p p}(T)=\frac{L_{p p}(t)}{P_{p}^{2} \cdot\left(X_{x}+p X_{u}\right)}
$$

and

$$
\widetilde{E}(\bar{L})(T)=\frac{\widetilde{E}(L)(t)}{P_{p} \cdot\left(X_{x}+p X_{u}\right)^{2}}+\frac{P_{x}+p P_{u}}{X_{x}+p X_{u}} \cdot \frac{L_{p p}(t)}{P_{p}^{2} \cdot\left(X_{x}+p X_{u}\right)}
$$

The two left hand sides in (5.32) and (5.33) only depend on target coordinates and are therefore invariant under the right action of $\mathcal{G}$. We can normalize both, setting the first to 1 and the second to zero. This gives

$$
X_{x}+p X_{u}=\frac{L_{p p}}{P_{p}^{2}}, \quad P_{x}+p P_{u}=-\frac{\widetilde{E}(L)}{L_{p p}} P_{p}
$$

where all sections are now being evaluated on the source coordinates $t=(x, u, p)$. Recalling the first order integrability condition $X_{x}+p X_{u}=\frac{U_{u}}{P_{p}}$ we also have on this partial moving frame that

$$
\frac{U_{u}}{P_{p}}=\frac{L_{p p}}{P_{p}^{2}} \Rightarrow U_{u}=\frac{L_{p p}}{P_{p}}
$$

and the lifted horizontal coframe (which is a basis for the restricted Maurer-Cartan forms on $\mathcal{G}_{1}$ to this first order partial moving frame) is

$$
\begin{aligned}
\omega^{x} & =X_{x} d x+X_{u} d u=\frac{L_{p p}}{P_{p}^{2}} d x+X_{u}(d u-p d x)=\frac{U_{u}^{2}}{L_{p p}} d x+X_{u}(d u-p d x), \\
\omega^{u} & =-p U_{u} d x+U_{u} d u=U_{u}(d u-p d x), \\
\omega^{p} & =P_{x} d x+P_{u} d u+P_{p} d p=-\frac{\widetilde{E}(L)}{L_{p p}} P_{p} d x+P_{u}(d u-p d x)+P_{p} d p \\
& =\frac{1}{U_{u}} \eta_{c}+P_{u}(d u-p d x),
\end{aligned}
$$


where $\eta_{c}$ is the differential form

$$
\eta_{c}=-\tilde{E}(L) d x+L_{p p} d p
$$

We choose as group parameters

$$
a_{1}=X_{u}, a_{2}=U_{u}, a_{3}=P_{u}
$$

and as a base coframe $\left\{\eta^{1}, \eta^{2}, \eta^{3}\right\}=\left\{\frac{1}{L_{p p}} d x, d u-p d x, \eta_{c}\right\}$, but with these choices, we can write $\left\{\omega^{x}, \omega^{u}, \omega^{p}\right\}$ as

$$
\left[\begin{array}{l}
\omega^{x} \\
\omega^{u} \\
\omega^{p}
\end{array}\right]=\left[\begin{array}{ccc}
a_{2}^{2} & a_{1} & 0 \\
0 & a_{2} & 0 \\
0 & a_{3} & \frac{1}{a_{2}}
\end{array}\right]\left[\begin{array}{l}
\eta^{1} \\
\eta^{2} \\
\eta^{3}
\end{array}\right] .
$$

Remark 5.4.11. So far we have used the lift of the standard coordinate coframe $\{d x, d u, d p\}$ on the base space to obtain a right-invariant horizontal coframe on $\mathcal{B}_{1}$. Other choices of coframes on the base space can be useful in implementing Cartan's equivalence method. It is a simple matter to extend all our results on the termination of various (past and future) completion algorithms to the case where a different coframe from the coordinate one is chosen. Assume we have chosen a coframe $\boldsymbol{\eta}$ that is an invertible linear combination of the coordinate coframe,

$$
\boldsymbol{\eta}=A \cdot\left[\begin{array}{c}
d x^{1} \\
\vdots \\
d x^{n}
\end{array}\right]
$$

where $A$ is a $G L(n)$-valued function on $J^{\infty}(\mathcal{E})$. Then the lift of $\boldsymbol{\eta}$ is an invariant horizontal coframe,

$$
\lambda(\boldsymbol{\eta})=\lambda(A) \cdot\left[\begin{array}{c}
\omega^{1} \\
\vdots \\
\omega^{n}
\end{array}\right]
$$


and instead of computing $d \mu^{i}$ in step (a) of Algorithm 5.1.1, or $d \omega^{i}$ on $\mathcal{B}_{1}$, we instead compute $d \lambda(\boldsymbol{\eta})$, and so on. Since $\lambda(\boldsymbol{\eta})$ is an invertible linear combination of the $\omega^{i}$, Algorithm 5.1.1 will produce the same results independent on which horizontal coframe we are using.

We give two illustrating examples of the above remark.

Example 5.4.12. Consider the equivalence problem of non-closed two-forms on open sets in $\mathbb{R}^{3}$, i.e. when are two two-forms $\Omega$ and $\bar{\Omega}$ related under the pull-back of a local diffeomorphism, $\varphi^{*} \bar{\Omega}=\Omega$. We could set this problem up in our usual framework by having $\mathcal{G}$ be the pseudo-group of local diffeomorphisms on $\mathbb{R}^{3}$ in coordinates $t=(x, y, z)$. Then, writing two forms $\Omega$ in the coordinate basis,

$$
\Omega=A d x \wedge d y+B d y \wedge d z+C d z \wedge d x
$$

a transformation $\varphi \in \mathcal{G},(x, y, z) \stackrel{\varphi}{\mapsto}(X, Y, Z)$, pulls $\Omega$ back according to

$$
\begin{aligned}
\varphi^{*} \Omega_{\left.\right|_{\varphi(t)}} & =A(T)\left(X_{x} Y_{y}-X_{y} Y_{x}\right) d x \wedge d y+B(T)\left(Y_{x} Z_{y}-Y_{y} Z_{x}\right) d x \wedge d y \\
& +C(T)\left(Z_{x} X_{y}-Z_{y} X_{x}\right) d x \wedge d y+\cdots
\end{aligned}
$$

where we have omitted the coefficients of $d y \wedge d z$ and $d z \wedge d x$. From these equations we may deduce the action of $\mathcal{G}$ on the coefficients of $\Omega$ but this action is obviously rather complicated. This difficulty can be avoided by a more clever choice of base coframe. In fact, the two-form $\Omega$ can be written as a single wedge product of two one-forms (see [36]),

$$
\Omega=\eta^{1} \wedge \eta^{2}
$$

Completing these to a coframe by some one-form $\eta^{3}$ such that $\eta^{1} \wedge \eta^{2} \wedge \eta^{3} \neq 0$ we can see that $\varphi \in \mathcal{G}$ preserves $\Omega$ if and only if

$$
\varphi^{*}\left[\begin{array}{l}
\eta^{1} \\
\eta^{2} \\
\eta^{3}
\end{array}\right]=\left[\begin{array}{lll}
a_{1} & a_{2} & 0 \\
a_{3} & a_{4} & 0 \\
c_{1} & c_{2} & c_{3}
\end{array}\right] \cdot\left[\begin{array}{l}
\eta^{1} \\
\eta^{2} \\
\eta^{3}
\end{array}\right], \quad \text { where } a_{1} a_{4}-a_{2} a_{3}=1 \text { and } c_{3} \neq 0
$$


for functions $a_{i}$ and $c_{j}$. To explain where this $G$-structure appears in our framework consider the following. The coefficients of $\eta^{1}$ and $\eta^{2}$ in the coordinate coframe are somewhat complicated expressions in the $A, B$ and $C$ from (5.35). A general local diffeomorphism $\varphi \in \mathcal{G}$ pulls $\Omega$ back to

$$
\varphi^{*} \Omega=\tilde{A} \eta^{1} \wedge \eta^{2}+\tilde{B} \eta^{2} \wedge \eta^{3}+\tilde{C} \eta^{3} \wedge \eta^{1}
$$

where, when everything is unraveled, $\tilde{A}, \tilde{B}$ and $\tilde{C}$ are expressions in $A, B, C$ and $j^{1} \varphi$. Normalizing the lifted invariants $\tilde{A}, \tilde{B}$ and $\tilde{C}$ to 1,0 and 0 , respectively provides the partial moving frame on which (5.36) arises as the restricted lifted horizontal coframe when we choose the $\eta^{i}$ as base coframe. This shows how a choice of base coframe can hasten the normalization process. Cartan's equivalence method then proceeds symbolically, not writing out the forms $\eta^{i}$ in coordinates but taking the exterior derivative of the right hand side in (5.36). This process is explained in Section 5.6 below.

Example 5.4.13. Let $g=\sum_{1 \leq i, j \leq 2} g_{i j} d x^{i} d x^{j}$ be a Riemannian metric on an open set in $\mathbb{R}^{2}$. Letting $\mathcal{G}$ be the collection of local diffeomorphism on $\mathbb{R}^{2}$, we have seen how the components of the metric transform under $\varphi \in \mathcal{G}$ acting on the base space $\mathbb{R}^{2}$ in Example 4.1.4. Normalizing all the lifted invariants $j^{1} \varphi \cdot g_{i j}$ will give an invariant horizontal coframe on a partial moving frame, $\mathcal{B}_{1}$. Alternatively, we can diagonalize the metric, writing it in the form

$$
g=\left(\eta^{1}\right)^{2}+\left(\eta^{2}\right)^{2}
$$

for a certain choice of coframe $\eta^{i}$ on $\mathbb{R}^{2}$ (depending on the original components $\left.g_{i j}\right)$. Then $\varphi \in \mathcal{G}$ preserves $g$ if and only if

$$
\varphi^{*}\left[\begin{array}{l}
\eta^{1} \\
\eta^{2}
\end{array}\right]=h \cdot\left[\begin{array}{l}
\eta^{1} \\
\eta^{2}
\end{array}\right]
$$

where $h$ is an orthonormal matrix, $h \in S O(2)$. This $S O(2)$-structure arises from 
normalizing the lifted coefficients $A, B, C$ and $D$ in

$$
\begin{aligned}
& \varphi^{*} \eta^{1}=A \eta^{1}+B \eta^{2}, \\
& \varphi^{*} \eta^{2}=C \eta^{1}+D \eta^{2},
\end{aligned}
$$

such that

$$
\left[\begin{array}{ll}
A & B \\
C & D
\end{array}\right] \in S O(2),
$$

i.e. normalizing $A^{2}+B^{2}=C^{2}+D^{2}=1$ and $A C+B D=0$.

The most important point of the last two examples is that they show how the classic $G$-structures are special cases of the general construction of MaurerCartan forms of the diffeomorphism pseudo-group restricted to partial moving frames. Therefore, our general equivalence method (Algorithm 5.5.3 in the next subsection) will directly apply to the equivalence problem of $G$-structures that arise from horizontal actions.

\subsection{Structure of a partial moving frame}

To solve the equivalence problem of sections in a partial moving frame, we need to understand the structure of the (restrictions of) Maurer-Cartan forms thereon. In this subsection we relate this structure to the algebraic structure of the "congruence equations" $\mathcal{G}^{u \bar{u}}$ from Section 5.1. In particular, since $\mathcal{G}^{u \bar{u}}$ can be completed to involution by Algorithm 5.1.1, we shall prove that by successively normalizing lifted invariants we eventually obtain an involutive coframe on a partial moving frame. This will serve to prove termination of Cartan's equivalence method in the horizontal case. It should not come as a surprise that the structure of $\mathcal{G}^{u \bar{u}}$ and the "corresponding" (in a sense we explain below) partial moving frame are intimately related since both objects determine the congruence problem by Corollary 5.4.4. We delay any discussion of regularity until after presenting our partial moving frame algorithm below. 
We have assumed that the zero order target coordinates can always be normalized, $X \equiv b$, and have seen that this reduces the zero order Maurer-Cartan forms $\mu^{i}$ to the lifted horizontal coframe $\omega^{i}$. Once we further restrict the lifted $U^{\alpha}$ to be constants, $c^{\alpha}$, there appear certain linear dependencies among the first order Maurer-Cartan forms. The recurrence formula tells us that on this partial moving frame

$$
0=d c^{\alpha}=d U^{\alpha}=U_{i}^{\alpha} \omega^{i}+\sum_{|K| \leq 1} \lambda\left(\left.\frac{\partial U^{\alpha}}{\partial X_{K}^{i}}\right|_{\mathbb{1}}\right) \mu_{K}^{i}+\text { contact forms on } J^{\infty}(\mathcal{E})
$$

When we restrict the partial moving frame $\widetilde{\mathcal{B}}_{1}$ to a specific section $j^{\infty} u$, and obtain the space $\widetilde{\mathcal{B}}_{1}^{u}$, the contact forms on $J^{\infty}(\mathcal{E})$ vanish and we have

$$
0=\left.d U^{\alpha}\right|_{u}=U_{i{ }_{u}}^{\alpha} \omega^{i}+\sum_{|K| \leq 1} \lambda\left(\left.\frac{\left.\partial U^{\alpha}\right|_{u}}{\partial X_{K}^{i}}\right|_{\mathbb{1}}\right) \mu_{K}^{i}
$$

Now compare this to the linear dependencies that appear when applying Algorithm 5.1.1 to $\mathcal{G}^{u \bar{u}}$ which is determined by the equations for $\mathcal{G}$ and the equations $U_{\left.\right|_{u}}=\bar{u}(X)$. We have, on $\mathcal{G}_{1}^{u \bar{u}}$, that

$$
0=d\left(U_{\left.\right|_{u}}^{\alpha}-\bar{u}^{\alpha}(X)\right)=d U_{\left.\right|_{u}}^{\alpha}-\left(\bar{u}_{i}^{\alpha}(X) \omega^{i}+\bar{u}_{i}^{\alpha}(X) \mu^{i}\right) .
$$

Comparing (5.37) with (5.38) we can see that the difference is in the term $\bar{u}_{i}^{\alpha}(X) \omega^{i}+$ $\bar{u}_{i}^{\alpha}(X) \mu^{i}$. To find integrability conditions in $\mathcal{G}_{1,1}^{u \bar{u}^{(1)}}$, Algorithm 5.1.1 computes the purely horizontal parts of the set $d \mu^{i}$. Recalling that $d \mu^{i}=-d \omega^{i}=\omega^{j} \wedge \mu_{j}^{i}$ and comparing with (5.38), the coefficients of purely horizontal forms will be of the form

$$
\left.U_{i}^{\alpha}\right|_{u}-\bar{u}_{i}^{\alpha}(X)
$$

that are then set to zero and solved for parametric derivatives (but note Remark 5.5.1 below). On the other hand, restricting to $\widetilde{\mathcal{B}}_{1}^{u}$ and recalling (5.37), the horizontal parts will only involve $\left.U_{i}^{\alpha}\right|_{u}$ restricted to $X=b$ and $U^{\alpha}=c^{\alpha}$. These 
expressions are invariant and may be normalized to obtain a higher order partial moving frame $\widetilde{\mathcal{B}}_{1,1}^{u}$.

Say we normalize $\left.U_{i}^{\alpha}\right|_{u} \equiv c_{i}^{\alpha}$. Solving these normalization equations is obviously equivalent to searching for integrability conditions in $\left.U_{i}^{\alpha}\right|_{u}=\bar{u}_{i}^{\alpha}(X)$ at points in $\mathcal{G}_{1,1}^{u \bar{u}}$ with target coordinate $b$ and for a choice of section $\bar{u}=v$ such that

$$
v^{\alpha}(b)=c^{\alpha}, \quad v_{i}^{\alpha}(b)=c_{i}^{\alpha} .
$$

Note that this means that $v$ intersects the cross-section determining the partial moving frame. An integrability condition now appears when, after normalizing some of the $\left.U_{i}^{\alpha}\right|_{u}$, a lifted invariant $\left.U_{j}^{\beta}\right|_{u}$ is no longer second order, but first or zero order. If it happens to be first order it can be normalized and we must start over, computing $d \omega^{i}$ restricted to our new first order partial moving frame (see step (a) in Algorithm 5.1.1). If the invariant is zero order it represents a genuine invariant of the problem and must be preserved by equivalence maps. Normalizing $\left.U_{i}^{\alpha}\right|_{u}=c_{i}^{\alpha}$ introduces further linear dependencies among the Maurer-Cartan forms which, according to the recurrence formula, are

$$
0=d c_{i}^{\alpha}=\left.d U_{i}^{\alpha}\right|_{u}=U_{\left.i j\right|_{u}}^{\alpha} \omega^{j}+\sum_{|K| \leq 2} \lambda\left(\left.\frac{\left.\partial U_{i}^{\alpha}\right|_{u}}{\partial X_{K}^{i}}\right|_{\mathbb{I}}\right) \mu_{K}^{i} .
$$

Assume that no integrability conditions were found, i.e. that $\mathcal{G}_{1,1}^{u v^{(1)}}=\mathcal{G}_{1}^{u v}$. This is equivalent to there not appearing any invariants of order at most one during the normalization of $\left.U_{i}^{\alpha}\right|_{u}$. Next, compare the collection of first degree forms, used to compute reduced Cartan characters, on $\mathcal{G}_{1}^{u v}$ and $\widetilde{\mathcal{B}}_{1}^{u}$,

$$
\left.\left.\left.\left\{\gamma_{1}\left(a_{1}^{j} D_{X^{j}}\right\lrcorner d \mu^{i}\right)\right|_{\mathcal{G}_{1}^{u v}}\right\} \quad \text { and } \quad\left\{\gamma_{1}\left(a_{1}^{j} D_{X^{j}}\right\lrcorner d \mu^{i}\right){\mid \widetilde{\mathcal{B}}_{1}^{u}}\right\}
$$

Again, these two collections will agree when we evaluate the former at the point of the cross-section where $v$ intersects it. That is, points with target coordinate $X=b$, since there we have $v^{\alpha}(b)=c^{\alpha}$ and $v_{i}^{\alpha}(b)=c_{i}^{\alpha}$. Note also that the number 
of second order group parameters that we have not yet normalized is the same as the number of second order parametric derivatives for $\mathcal{G}_{1,1}^{u v}$ at the target coordinate $X=b$.

Remark 5.5.1. Of course, when we compute horizontal parts of $d \mu_{K}^{i}$, prolongations of the defining equations of $\mathcal{G}$ appear also. Since these have nothing to do with the choice of sections $j^{\infty} u$ and $j^{\infty} v$, these appear, independent of the normalizations made, when we work on a partial moving frame.

If the involutivity test based on $\left(5.40\right.$ for $\mathcal{G}_{1}^{u v}$ is successful we say that the partial moving frame $\widetilde{\mathcal{B}}_{1}^{u}$ is involutive. In general, if $\mathcal{B}_{p}^{u}$ is a partial moving frame of order $p$, and we compute $d \mu_{K}^{i}$, for all $|K|<p$, and normalize all purely horizontal parts, we denote the resulting partial moving frame by $\mathcal{B}_{p, 1}^{u}$. If we happen to normalize a group parameter of order strictly less than $p+1$ during this computation, we denote the $p^{\text {th }}$ order partial moving frame obtained by the projection $\sigma_{p}^{p+1}\left(\mathcal{B}_{p, 1}^{u}\right)$ by $\mathcal{B}_{p, 1}^{u^{(1)}}$. This is analogous to the notation used for differential equations. If we do not normalize any group parameter of order $\leq p$, then $\mathcal{B}_{p, 1}^{u^{(1)}}=\mathcal{B}_{p}^{u}$ and the number, $r^{(p+1)}$, of non-normalized group parameters of order $p+1$ is the same as the number of parametric derivatives of order $p+1$ for $\mathcal{G}_{p, 1}^{u v}$ where $v$ intersects the cross-section determining $\mathcal{B}_{p, 1}^{u}$. If the reduced Cartan characters obtained by successively maximizing the ranks of unions of collections of the form

$$
\left.\left\{\gamma_{p}\left(a_{1}^{j} D_{X^{j}}\right\lrcorner d \mu_{K}^{i}\right){\mid \widetilde{\mathcal{B}}_{p}^{u}}_{p}\right\}, \quad|K|<p,
$$

(again, these agree with the reduced Cartan characters of $\mathcal{G}_{p}^{u v}$ ) satisfy Cartan's involutivity test (i.e. $r^{(p+1)}=\sum i \tilde{s}_{i}^{(p)}$ ) we say that $\mathcal{B}_{p}^{u}$ is involutive. The next section discusses the significance of obtaining an involutive partial moving frame for the congruence problem.

Remark 5.5.2. In practice, when we construct a partial moving frame as above, we do not fix a specific function $u$, but rather work with the unspecified jets of such maps, $j^{\infty} u_{\left.\right|_{x}}=\left(x, u, \ldots, u_{J}^{\alpha}, \ldots\right)$. We then need to be careful to keep track 
of which choices of $u$ allow for which normalizations of the lifted invariants as this can differ from section to section, see Remark 5.2.7. If $J^{\infty}(\mathcal{E})=\cup_{1 \leq i \leq N} \mathcal{S}_{i}$ is the partition of $J^{\infty}(\mathcal{E})$ into the different domains of definitions of partial moving frames we split the equivalence problem into different branches where, in each branch, we consider only the congruence of sections $j^{\infty} u$ and $j^{\infty} \bar{u}$ are contained in the same set $\mathcal{S}_{i}$. Notice that two sections that lie in different sets $\mathcal{S}_{i}$ are never congruent since they can not be mapped to the same cross-section (if they were congruent this would not be the case). Notice also that if $j^{\infty} u$ intersects more than one set $\mathcal{S}_{i}$, we do not attempt to say anything about the congruence problem pertaining to $u$. This is because the equations $\mathcal{G}^{u \bar{u}}$ will be non-regular in the sense of Definition 3.0.6 and our methods break down, see next subsection.

Here then is our algorithm for the construction of a partial moving frame. We shall elaborate on its different steps in the following remarks. Recall that for a partial moving frame $\mathcal{B}_{t}^{u} \subset \widetilde{\mathcal{G}}_{t}$ we denoted its inverse image under $\tilde{\sigma}_{t}^{\infty}$ as $\widetilde{\mathcal{B}}_{t}^{u} \subset \widetilde{\mathcal{G}}_{\infty}$.

\section{Algorithm 5.5.3.}

(a) Normalize all zero order lifted invariants $X$ and $U^{\alpha}$ to obtain a partial moving frame $\mathcal{B}_{1}^{u}$. Compute all the exterior derivatives $d \omega^{i}, i=1, \ldots, n$, on $\widetilde{\mathcal{B}}_{1}^{u}$ and normalize all horizontal parts. If in that process we manage to normalize a first order group parameter, replace $\mathcal{B}_{1}^{u}$ by $\mathcal{B}_{1,1}^{u^{(1)}}$ and repeat this step. (If we happen to find a genuine invariant we refer to Remark 5.5.6.)

(b) When we stop being able to normalize first order group parameters we test for involution by computing the maximal ranks of (the union of) collections of the form

$$
\left.\left\{\gamma_{1}\left(a_{1}^{j} D_{X^{j}}\right\lrcorner d \omega^{i}\right)_{\widetilde{\mathcal{B}}_{1}}\right\}_{i=1, \ldots, n},
$$

and counting the number of group parameters of order 2 that have not been normalized. If the test fails, replace $\mathcal{B}_{1}^{u}$ by $\mathcal{B}_{2}^{u}=\mathcal{B}_{1,1}^{u}$ in step (a) and compute $d \mu_{j}^{i}$, for all parametric $X_{j}^{i}$, restricted to $\widetilde{\mathcal{B}}_{2}^{u}$, normalize horizontal parts and so on. 
(c) If, at some stage of this algorithm, when computing $\mathcal{B}_{p, 1}^{u^{(1)}}$, we manage to normalize a group parameter of some order $t<p+1$, we replace $\mathcal{B}_{p}^{u}$ by $\mathcal{B}_{t}^{u}:=\sigma_{t}^{p+1}\left(\mathcal{B}_{p, 1}^{u}\right)$, a $t^{\text {th }}$ order partial moving frame, and repeat step (a) using $\mathcal{B}_{t}^{u}$.

Remark 5.5.4 (Regularity of partial moving frames). As always, local solvability of differential equations can only be guaranteed for sufficiently regular equations. In order for us to eventually solve the equivalence problem of sections using Algorithm 5.5 .3 we need to assume, first of all, that every partial moving frame $\mathcal{B}_{t}^{u}$ is a manifold for all $u$ in the domain of definition of $\mathcal{B}_{t}$. Second, we require that the reduced Cartan characters computed in step (b) of Algorithm 5.5.3 are constant on the partial moving frames $\mathcal{B}_{t}^{u}$. As in the case of Algorithm 3.2.6, these regularity hypothesis guarantee termination of the algorithm and we henceforth assume they hold for our partial moving frames. Equivalence problems of constant type, see [36], are trivially regular, since the structure equations for the partial moving frames for these problems have constant coefficients at top order and so the reduced Cartan characters found in (5.41) will be constant. This shows that our algorithm is more general, but one must still be careful to check that the regularity of Definition 3.0 .6 holds.

Remark 5.5.5. Let us prove that if $u$ and $\bar{u}$ are two functions whose graphs are congruent under the action of $\mathcal{G}$ then the sequences of moving frames $\mathcal{B}_{t}^{u}$ and $\mathcal{B}_{t}^{\bar{u}}$, computed by Algorithm 5.5.3, reach involution at the same time. Let $\psi \in \mathcal{G}$ map $u$ to $\bar{u}$. Then, for every section $s$ of $\mathcal{B}_{t}^{u}$ we have that $\bar{s}=R_{\psi} \cdot s$ is a section of $\mathcal{B}_{t}^{\bar{u}}$. Since $R_{\psi}$ is a diffeomorphism on its domain of definition in $\mathcal{G}_{t}$, we have that $R_{\psi}: \mathcal{B}_{t}^{u} \rightarrow \mathcal{B}_{t}^{\bar{u}}$ is a diffeomorphism and hence that the structure equations of the restricted Maurer-Cartan forms on $\mathcal{B}_{t}^{u}$ and $\mathcal{B}_{t}^{\bar{u}}$ are isomorphic. Since the reduced Cartan characters are determined by the structure equations, the sequences of $\mathcal{B}_{t}^{u}$ and $\mathcal{B}_{t}^{\bar{u}}$ will reach involution at the same time.

Remark 5.5.6. When we stumble upon a genuine invariant for our problem, it represents a zero order equation in $\mathcal{G}^{u v}$. That is, if $I\left(x, j^{p} u_{\left.\right|_{x}}\right)$ is the invariant, 
then the corresponding equation in $\mathcal{G}^{u v}$ is of the form

$$
I\left(x, j^{p} u_{\left.\right|_{x}}\right)=I\left(X, j^{p} v_{\left.\right|_{X}}\right)
$$

In accordance with Remark 3.0.3, we need to prolong these equations and test them for integrability conditions. Say we find a genuine invariant $I$ when computing $\mathcal{B}_{p, 1}^{u^{(1)}}$, so that 5.42 is found when computing $\mathcal{G}_{p, 1}^{u v^{(1)}}$. Prolonging 5.42 using differentiation with respect to the target variables gives

$$
D_{X^{i}} I\left(x, j^{p} u_{\left.\right|_{x}}\right)=\frac{\partial I\left(X, j^{p} v_{\left.\right|_{X}}\right)}{\partial X^{i}}
$$

The right hand sides are always zero order. Since $I\left(x, j^{p} u_{\left.\right|_{x}}\right)$ is preserved by equivalence maps of sections in $\mathcal{B}_{p}^{u}$, so is its exterior derivative, $d I\left(x, j^{p} u_{\left.\right|_{x}}\right)$, but since there are no group parameters present in $I\left(x, j^{p} u_{\left.\right|_{x}}\right)$ the exterior derivative of $I$ will only involve the horizontal forms $\omega^{i}$, and the left hand sides in (5.43) as the coefficients. Hence, by applying the exterior derivative (perhaps repeatedly) to any genuine invariant that we obtain in the process of building a partial moving frame, we recover the corresponding prolonged equations in $\mathcal{G}^{u v}$.

If the coefficients of $d I=I_{i} \omega^{i}$ (or the "iterated" coefficients $d I_{i}=I_{i ; j} \omega^{j}$ ) involve first order group parameters we can normalize them to obtain a smaller, first order, partial moving frame. By step (c) in Algorithm 5.5.3 we then repeat step (a) with this partial moving frame. The coefficients that do not involve group parameters are further genuine invariants of the problem (representing zero order equations in $\mathcal{G}^{u v}$ ) that must be prolonged also. Say we have obtained a collection $I_{w}(x)=I_{w}\left(x, j^{\infty} u_{\left.\right|_{x}}\right)$, for $w$ in some index set $W$, of genuine invariants by repeatedly applying the exterior derivative on $\mathcal{B}_{p}^{u}$. Assume that at the next step, when computing the exterior derivatives

$$
d I_{w}(x)=I_{w ; i}(x) \omega^{i}
$$

it turns out that the $I_{w ; i}$ are functions of $x$ only, and are functionally dependent 
on the $I_{w}(x)$, i.e. there are functions $H^{w ; i}$ such that

$$
I_{w ; i}(x)=H^{w ; i}\left(\left\{I_{l}(x)\right\}_{l \in W}\right) .
$$

Then we can actually stop applying the exterior derivatives to genuine invariants, since, by the chain rule, we have

$$
d I_{w ; i}=\frac{\partial H^{w ; i}}{\partial I_{l}} I_{l ; j} \omega^{j}
$$

and each new coefficient is a function of genuine invariants already obtained. Once this happens (note that it will happen since there cannot be more than $\operatorname{dim} \mathcal{X}$ genuine invariants) we continue with Algorithm 5.5.3. We collect the invariants

$$
\left\{I_{w}, I_{w, i}\right\}_{w \in \bigcup W_{t}, 1 \leq i \leq n}
$$

(note that we may have more than one index sets $W_{t}$ ) found by Algorithm 5.5.3 in a map $\Sigma^{u}: \operatorname{dom} u \rightarrow \mathbb{R}^{A}$, called the signature map of $u$. The image $\Sigma^{u}(\operatorname{dom} u)$ is called the signature of $u$. Once we stop getting new, functionally independent genuine invariants we say that the signature has stabilized. In general, the signatures for different functions $u$ and $\bar{u}$ stabilize at different times, but if $u$ and $\bar{u}$ are to be congruent, by Corollary 5.4.4, their signatures must overlap,

$$
\Sigma^{u}(\operatorname{dom} u)=\Sigma^{\bar{u}}(\operatorname{dom} \bar{u})
$$

Remark 5.5.7. If we manage to normalize all first order group parameters, our partial moving frame reduces to a (proper) moving frame $\rho$, and pulling back the Maurer-Cartan forms to $\mathcal{X}$ gives a coframe (and possibly some genuine invariants) on the base space $\mathcal{X}$, namely $\omega^{1}, \ldots, \omega^{n}$ pulled back by the moving frame. The equivalence problem has now been reduced to an equivalence problem of coframes on $\mathcal{X}$. Cartan solved the equivalence problem for coframes, [7], according to which a generating set of invariants for the problem is given by the genuine invariants 
found during Algorithm 5.5.3 and the structure functions of the invariant coframe $\omega^{i}$, i.e. the coefficients in

$$
d \omega^{i}=R_{j k}^{i} \omega^{j} \wedge \omega^{k}
$$

Remark 5.5.8. Regarding the two previous remarks, it should be said that equivalence problems in which we find genuine invariants before we manage to normalize all first order group parameters are rare. These equivalence problems are said to be of non-constant type since they may introduce some non-constant coefficients of the top order terms in the structure equations. The analysis of such equivalence problems is a little bit more problematic since they make it slightly more difficult to verify regularity of the equations $\mathcal{G}_{\infty}^{u v}$ and hence of the equivalence problem. However, since we can still recover the defining equations of the differential equations $\mathcal{G}_{\infty}^{u v}$, Algorithm 5.5.3 will still terminate at involution at the same time as Algorithm 5.1.1 for $\mathcal{G}_{\infty}^{u v}$, and there is no need to panic and abandon your problem.

Remark 5.5.9. Expanding on Remark 5.5.7, assume that after normalizing all horizontal parts in the structure equations for a partial moving frame $\widetilde{\mathcal{B}}_{p}^{u}$, we notice we have normalized all group parameters of order $p+1$. Note that this happens when the corresponding congruence equation $\mathcal{G}_{p+1}^{u v}$ is maximally over-determined at order $p+1$. In this case, $\mathcal{B}_{p}^{u}$ and $\mathcal{B}_{p+1}^{u}:=\mathcal{B}_{p, 1}^{u}$ have equal dimension and the Maurer-Cartan forms $\mu_{K}^{i},|K| \leq p$ on $\mathcal{B}_{p+1}^{u}$ form a bona-fide coframe for $\mathcal{B}_{p+1}^{u}$, for any function $u$ in the domain of definition of the partial moving frame. Let $u$ and $\bar{u}$ be in this domain and let $\Lambda: \mathcal{B}_{p+1}^{u} \rightarrow \mathcal{B}_{p+1}^{\bar{u}}$ be any diffeomorphism that preserves the Maurer-Cartan forms. Letting $s$ be a section of $\mathcal{B}_{p+1}^{u} \stackrel{\sigma}{\rightarrow}$ dom $u$ we have, since $\Lambda$ preserves $\omega^{i}$, that the map

$$
f(x):=\sigma\left(\Lambda\left(s_{\mid x}\right)\right)
$$

is a local diffeomorphism from dom $u$ to dom $\bar{u}$ (see proof of Corollary 5.3.5). Now let $\bar{s}$ be the image of $s$ under $\Lambda$, i.e. $\bar{s}_{\left.\right|_{f(x)}}=\Lambda\left(\left.s\right|_{x}\right)$. Since the action of $\Lambda$ on the source coordinate is a diffeomorphism, $\bar{s}$ is also a section and since $\Lambda$ preserves the Maurer-Cartan forms $\mu_{K}^{i},|K|<p+1$, so does its restriction $\Lambda: s \rightarrow \bar{s}$. By 
our fundamental Theorem 5.3.4 $\Lambda$ must agree with the action of $R_{f}$ on $s$ and since this holds for any section $s$, we have

$$
\Lambda(g)=R_{f} \cdot g, \quad g \in \mathcal{B}_{p+1}
$$

Thus, when we manage to normalize all group parameters of some order $p+1$, we can reduce our equivalence problem of sections to an equivalence problem of coframes (on $\mathcal{B}_{p+1}^{u}$ and $\mathcal{B}_{p+1}^{\bar{u}}$ ), to which we can apply Cartan's solution. If the structure functions of the coframe on $\mathcal{B}_{p+1}^{u}$ include non-normalized group parameters, we may normalize them and continue with Algorithm 5.5.3. But if the structure functions are all constant, then $\mathcal{B}_{p+1}^{u}$ has a local Lie group structure. It is then well known that the diffeomorphisms $\Lambda$ preserving the coframe are the Lie group elements, and, in particular, the symmetry group of the section $u$ is this Lie group.

Definition 5.5.10. When Algorithm 5.5.3 normalizes all group parameters of some order $p+1$, and all structure functions, and their iterated derivatives, of the resulting coframe on $\mathcal{B}_{p+1}^{u}$ are genuine invariants (this includes constants), so no more normalizations happen, we say that the equivalence problem is fully determinate at order $p+1$.

Remark 5.5.11. A generating set of invariants for an equivalence problem is a collection of invariants such that any invariant of the problem is a function of the generating set and its invariant differentiation, i.e. differentiation with respect to the dual horizontal frame $D_{X^{i}}=\frac{\partial}{\partial \omega^{i}}$. An important feature of Algorithm 5.5.3 is that if the equivalence algorithm terminates with an invariant horizontal coframe, so all first order group parameters have been normalized, a generating set of invariants is apparent: It consists of all genuine invariants found during the implementation of the algorithm plus the structure functions of the horizontal coframe. The same obviously holds in fully determinate cases.

In general we have the following theorem for a regular equivalence problem 
(meaning that all the equations $\mathcal{G}_{t}^{u v}$ that appear during Algorithm 5.5.3 are regular).

Theorem 5.5.12. Algorithm 5.5.3 terminates either with a fully determinate partial moving frame or at involution. Moreover, it will do so at the same time as Algorithm 5.1.1 does for $\mathcal{G}_{\infty}^{u v}$, where $v$ intersects the cross-section determining the partial moving frame.

What does involution of a partial moving frame imply for the equivalence problem of sections thereof? In the next subsection, by applying the CartanKähler theorem, we prove that it leads to its complete solution.

\subsection{Exterior differential systems}

As shown in Section 5.4 and Remark 5.5.6, when $\mathcal{G}_{p}$ is formally integrable, the equivalence problem of two local functions, $u$ and $\bar{u}$, can be reduced to that of finding two sections of a partial moving frame $\mathcal{B}_{p}$ (restricted to $j^{\infty} u$ and $j^{\infty} \bar{u}$ ), $s$ and $\bar{s}$, and a local transformation, $\varphi$, of the base manifold $\mathcal{X}$ such that

$$
\varphi^{*} \bar{s}^{*} \mu_{K}^{i}=s^{*} \mu_{K}^{i}, \text { for all }|K|<p \text { and } \varphi^{*} \Sigma^{\bar{u}}=\Sigma^{u}
$$

We can recast this as a problem of finding an integral manifold for an exterior differential system. To see how, let $j^{\infty} u$ and $j^{\infty} \bar{u}$ be two sections of $J^{\infty}(\mathcal{E})$ in the same domain of definition of a partial moving frame $\mathcal{B}_{p}$ and let $\mathcal{B}_{p}^{u}$ and $\mathcal{B}_{p}^{\bar{u}}$ be involutive. Let the signatures of $u$ and $\bar{u}$ overlap (cf. Remark 5.5.6). Denote (the restriction of) the Maurer-Cartan forms on $\mathcal{B}_{p}^{u}$ by $\mu_{K}^{i}$, but those on $\mathcal{B}_{p}^{\bar{u}}$ by $\bar{\mu}_{K}^{i}$ (note that here we are only taking those $\mu_{K}^{i}$ with $|K|<p$ ). Let $V:=\operatorname{dom} u$ and $\bar{V}:=\operatorname{dom} \bar{u}$ and note that $\mathcal{B}_{p}^{u}$ is a product space

$$
\mathcal{B}_{p}^{u}=V \times \mathcal{H}_{p}^{u}
$$


where $\mathcal{H}_{p}^{u}$ is parametrized by non-normalized group parameters. Similarly, write $\mathcal{B}_{p}^{\bar{u}}=\bar{V} \times \overline{\mathcal{H}}_{p}^{u}$. Also, let $\gamma_{V \times \bar{V}}: \mathcal{B}_{p}^{u} \times \mathcal{B}_{p}^{\bar{u}} \rightarrow V \times \bar{V}$ be the projection onto the product of the domains of $u$ and $\bar{u}$. Consider an $n$-dimensional submanifold, $\mathcal{N}$, of $\mathcal{B}_{p}^{u} \times \mathcal{B}_{p}^{\bar{u}}$ which satisfies the following.

$$
\begin{aligned}
\left.\left(\mu_{K}^{i}-\bar{\mu}_{K}^{i}\right)\right|_{\mathcal{N}} & =0, \quad|K|<p, \\
\left.\left(\Sigma^{u}(x)-\Sigma^{\bar{u}}(\bar{x})\right)\right|_{\mathcal{N}} & =0, \\
\left.\omega^{1} \wedge \omega^{2} \wedge \cdots \wedge \omega^{n}\right|_{\mathcal{N}} & \neq 0 .
\end{aligned}
$$

The last condition is to ensure that the projection, $\gamma_{V \times \bar{V}}(\mathcal{N})$, of $\mathcal{N}$ is locally the graph of a (local) diffeomorphism $\varphi: V \rightarrow \bar{V}$. Given 5.47) we can parametrize $\mathcal{N}$ locally by

$$
x \mapsto(x, s(x), \varphi(x), \bar{s}(\varphi(x))),
$$

and (5.47) is equivalent to (5.46), as is easily checked. In practice, when genuine invariants are present, we first restrict to the subspace of $\mathcal{B}_{p}^{u} \times \mathcal{B}_{p}^{\bar{u}}$ on which $\Sigma^{u}(x)-$ $\Sigma^{\bar{u}}(\bar{x})=0$ and then ask whether we can construct, on this smaller space, an integral submanifold $\mathcal{N}$ satisfying

$$
\begin{array}{r}
\left.\left(\mu_{K}^{i}-\bar{\mu}_{K}^{i}\right)\right|_{\mathcal{N}}=0, \quad|K|<p, \\
\left.\omega^{1} \wedge \omega^{2} \wedge \cdots \wedge \omega^{n}\right|_{\mathcal{N}} \neq 0, \quad|K|<p .
\end{array}
$$

To construct such an integral manifold we need to understand the structure equations of $\mu_{K}^{i},|K|<p$, on $\mathcal{B}_{p}$. Recall the structure equations of the MaurerCartan forms on the infinite bundle $\mathcal{D}_{\infty}(\mathcal{X}), 4$.10). We have previously restricted these equations to the subspaces $\widetilde{\mathcal{B}}_{p}^{u}=\left(\sigma_{p}^{\infty}\right)^{-1}\left(\mathcal{B}_{p}^{u}\right)$ of the infinite bundle $\mathcal{G}_{\infty}$ but when solving (5.48) we are confined to a finite order partial moving frame $\mathcal{B}_{p}$ and must set each $p+1$ order group parameter still present in 4.10 to zero. We emphasize that since $\mathcal{B}_{p}^{u}$ is involutive we may have (during the last loop through Algorithm 5.5.3 already normalized some of the $p+1$ order group parameters. 
Setting non-normalized group parameters of order $p+1$ to zero will obviously only affect the top order Maurer-Cartan forms in 4.10 but we shall write $\alpha_{K, j}^{i}$ for the differential form we obtain by setting every not-yet-normalized $p+1$ order group parameter to zero in $\mu_{K, j}^{i}$ on $\widetilde{\mathcal{B}}_{p}^{u}$, and refer to them as truncated Maurer-Cartan forms. The structure equations on $\mathcal{B}_{p}^{u}$ are now the restrictions of 4.10 to $\widetilde{\mathcal{B}}_{p}^{u}$ where we replace the top order Maurer-Cartan forms by the $\alpha_{K, j}^{i}$. Let us continue our Example 5.4.1 to make this a little clearer.

Example 5.6.1. We note that the defining equations for $\mathcal{G}_{1}$ are formally integrable so we need not worry about integrability conditions in $\mathcal{G}$. The structure equations on $\mathcal{B}_{1}$ determined by $X=U=P=Q=0$ are obtained from those on $\widetilde{\mathcal{B}}_{1}$

$$
\begin{aligned}
& d \omega^{x}=\mu_{x}^{x} \wedge \omega^{x}, \\
& d \omega^{u}=\mu_{x}^{u} \wedge \omega^{x}+\mu_{u}^{u} \wedge \omega^{u}=\omega^{p} \wedge \omega^{x}+\mu_{u}^{u} \wedge \omega^{u}, \\
& d \omega^{p}=\left(-Q_{i}\right) \omega^{i} \wedge \omega^{x}+\mu_{u}^{p} \wedge \omega^{u}+\left(\mu_{u}^{u}-\mu_{x}^{x}\right) \wedge \omega^{p},
\end{aligned}
$$

by setting every second order group parameter to zero. Doing so will result in equations

$$
\begin{aligned}
& d \omega^{x}=\alpha_{x}^{x} \wedge \omega^{x}, \\
& d \omega^{u}=\omega^{p} \wedge \omega^{x}+\alpha_{u}^{u} \wedge \omega^{u}, \\
& d \omega^{p}=\left(-\widetilde{Q}_{i}\right) \omega^{i} \wedge \omega^{x}+\alpha_{u}^{p} \wedge \omega^{u}+\left(\alpha_{u}^{u}-\alpha_{x}^{x}\right) \wedge \omega^{p},
\end{aligned}
$$

where $\widetilde{Q}_{i}$ are the truncated lifted invariants (easily found by MATHEMATICA)

$$
\widetilde{Q}_{U}=-\frac{q_{p} P_{u}}{U_{u} X_{x}}-\frac{P_{u}^{2}}{U_{u}^{2}}+\frac{q_{u}}{X_{x}^{2}} \text { and } \widetilde{Q}_{P}=\frac{q_{p}}{X_{x}}+\frac{P_{u}}{U_{u}}
$$

The original Cartan equivalence method computes [5.50], see [36]. Conversely, expanding each $\alpha$ in 5.50 as the sum of a horizontal and a contact form on $\widetilde{\mathcal{B}}_{1}$ will produce (5.49).

We proceed with Algorithm 5.5.3 and set $Q_{U}$ and $Q_{P}$ to zero, normalizing $P_{u x}$ and $U_{u u}$ (sparing the reader any details of these normalizations for now). Note that we need not concern ourselves with $Q_{X}$ as $\omega^{x} \wedge \omega^{x}=0$ in (5.49) (cf. Remark 
3.2.3. . On $\widetilde{\mathcal{B}}_{1,1}$ we then have

$$
\begin{aligned}
& d \omega^{x}=\mu_{x}^{x} \wedge \omega^{x}, \\
& d \omega^{u}=\mu_{x}^{u} \wedge \omega^{x}+\mu_{u}^{u} \wedge \omega^{u}=\omega^{p} \wedge \omega^{x}+\mu_{u}^{u} \wedge \omega^{u} \\
& d \omega^{p}=\mu_{u}^{p} \wedge \omega^{u}+\left(\mu_{u}^{u}-\mu_{x}^{x}\right) \wedge \omega^{p} .
\end{aligned}
$$

But, when confined to $\mathcal{B}_{1,1}^{(1)}$, we must set each second order group parameter to zero. Doing so will simply give

$$
\begin{aligned}
& d \omega^{x}=\alpha_{x}^{x} \wedge \omega^{x}, \\
& d \omega^{u}=\omega^{p} \wedge \omega^{x}+\alpha_{u}^{u} \wedge \omega^{u} \\
& d \omega^{p}=\alpha_{u}^{p} \wedge \omega^{u}+\left(\alpha_{u}^{u}-\alpha_{x}^{x}\right) \wedge \omega^{p},
\end{aligned}
$$

where $\alpha_{u}^{p}, \alpha_{x}^{x}$ and $\alpha_{u}^{u}$ may have been augmented by the normalizations $Q_{U}=$ $Q_{P}=0$ but whose precise formulas are not needed. Indeed, the symbolic computations that the equivariant moving frame provides are among its most useful features. The original equivalence method finds these same differential forms, but the "lifted" equations (5.49) are obtained by expanding the truncated forms $\alpha_{j}^{i}$ in (5.50) as a sum of horizontal and contact forms. Since integrability conditions/invariants will appear as coefficients of purely horizontal parts, the original method replaced each

$$
\alpha_{j}^{i} \mapsto z_{j ; k}^{i} \omega^{k}
$$

and plugged this into (5.50). Taking only horizontal parts gives

$$
\begin{aligned}
& d \omega^{x} \mapsto z_{x ; k}^{x} \omega^{k} \wedge \omega^{x}, \\
& d \omega^{u} \mapsto \omega^{p} \wedge \omega^{x}+z_{u ; k}^{u} \omega^{k} \wedge \omega^{u}, \\
& d \omega^{p} \mapsto\left(-\widetilde{Q}_{i}\right) \omega^{i} \wedge \omega^{x}+z_{u ; k}^{p} \omega^{k} \wedge \omega^{u}+\left(z_{u ; k}^{u}-z_{x ; k}^{x}\right) \omega^{k} \wedge \omega^{p} .
\end{aligned}
$$

Each horizontal part is an invariant (on $\widetilde{\mathcal{B}}_{1}$ ) and may be normalized as we see fit. These horizontal parts were referred to as torsion coefficients in the classical 
method, and normalizing them was called absorption, [36]. However, most of these equations are simple prolongations of the first order defining equations for $\mathcal{G}$ and our knowledge of these cuts down the computational load of solving these absorption equations. Notice however that the number of parameters $z_{j ; k}^{i}$ that can not be normalized in (5.52) is equal to the number of second order group parameters that can not be normalized in (5.49). This is simply because these are two ways of computing the same thing (cf. Remark 3.2.8).

For simplicity, we shall focus on a first order problem in the following. So assume that $\mathcal{B}_{1}^{u}$ and $\mathcal{B}_{1}^{\bar{u}}$ are involutive. Also assume we have restricted to the subspace $\mathcal{F} \subset \mathcal{B}_{1}^{u} \times \mathcal{B}_{1}^{\bar{u}}$ on which the signatures overlap (if this is not possible then $u$ and $\bar{u}$ are not congruent and the game is over). An integral element, $E$, of the exterior differential system (5.48) at a point $(g, h) \in \mathcal{F}$ is an $n$-dimensional subspace of the tangent space $T_{(g, h)} \mathcal{F}$ such that the differential ideal generated by the one forms in 5.48 vanishes once restricted to $E$. This is equivalent to the generating one-forms and their exterior derivatives to vanish on $E$. That is

$$
\begin{array}{r}
\left(\omega^{i}-\bar{\omega}^{i}\right)_{\left.\right|_{E}}=0, \\
\left(d \omega^{i}-d \bar{\omega}^{i}\right)_{\left.\right|_{E}}=0, \\
\left.\omega^{1} \wedge \omega^{2} \wedge \cdots \wedge \omega^{n}\right|_{E} \neq 0 .
\end{array}
$$

The (EDS version of the) Cartan-Kähler theorem tells us when there is a local integral manifold with tangent space equal to $E$ at one point, see [36, Theorem 15.12]. Let us write the structure equations of $\omega^{i}$ on $\mathcal{B}_{1}^{u}$ as

$$
d \omega^{i}=A_{\kappa, j}^{i} \alpha^{\kappa} \wedge \omega^{j}+B_{j, k}^{i} \omega^{j} \wedge \omega^{k}
$$

where $A_{\kappa, j}^{i}$ and $B_{j, k}^{i}$ are genuine invariants (since each coefficient in the recurrence formula is invariant) depending on $j^{\infty} u$, and $\alpha^{\kappa}, \kappa=1, \ldots, r^{(1)}$ are the non-normalized, truncated (and augmented by absorption/normalization in $\mathcal{B}_{1,1}^{u}$ ) Maurer-Cartan forms $\mu_{j}^{i}$. Similarly, and since $u$ and $\bar{u}$ land in the domain of 
definition of the same partial moving frame, we have on $\mathcal{B}_{1}^{\bar{u}}$,

$$
d \bar{\omega}^{i}=\bar{A}_{\kappa, j}^{i} \bar{\alpha}^{\kappa} \wedge \bar{\omega}^{j}+\bar{B}_{j, k}^{i} \bar{\omega}^{j} \wedge \bar{\omega}^{k}
$$

for invariant coefficient functions $\bar{A}$ and $\bar{B}$ that agree with the $A$ 's and $B$ 's since we have restricted to the subspace $\mathcal{F}$ where the signatures overlap. We note that the $A$ 's and $B$ 's are constant unless we are in the rare case of an equivalence problem of non-constant type. We have, on $\mathcal{F}$,

$$
\begin{aligned}
d \omega^{i}-d \bar{\omega}^{i} & =A_{\kappa, j}^{i} \alpha^{\kappa} \wedge \omega^{j}+B_{j, k}^{i} \omega^{j} \wedge \omega^{k}-\bar{A}_{\kappa, j}^{i} \bar{\alpha}^{\kappa} \wedge \bar{\omega}^{j}-\bar{B}_{j, k}^{i} \bar{\omega}^{j} \wedge \bar{\omega}^{k} \\
& =A_{\kappa, j}^{i}\left(\alpha^{\kappa}-\bar{\alpha}^{\kappa}\right) \wedge \omega^{j}+B_{j, k}^{i}\left(\omega^{j}-\bar{\omega}^{j}\right) \wedge \omega^{k}+A_{\kappa, j}^{i} \bar{\alpha}^{\kappa} \wedge\left(\omega^{j}-\bar{\omega}^{j}\right) \\
& +B_{j, k}^{i} \bar{\omega}^{j} \wedge\left(\omega^{k}-\bar{\omega}^{k}\right) .
\end{aligned}
$$

We can use the first set of equations in 5.53 to eliminate most of the terms in (5.56) and (5.53) is seen to be equivalent to

$$
\begin{aligned}
\left.\left(\omega^{i}-\bar{\omega}^{i}\right)\right|_{E} & =0, \\
\left.\left(A_{\kappa, j}^{i}\left(\alpha^{\kappa}-\bar{\alpha}^{\kappa}\right) \wedge \omega^{j}\right)\right|_{E} & =0, \\
\left.\omega^{1} \wedge \omega^{2} \wedge \cdots \wedge \omega^{n}\right|_{E} & \neq 0 .
\end{aligned}
$$

The reduced Cartan characters for the EDS (5.47) are defined successively by first setting

$$
\left.\tilde{s}_{1}^{(1)}=\max \operatorname{rank}\left\{\mathcal{C}\left(v_{1}\right)\right\}=\max \operatorname{rank}\left\{\gamma_{1}\left(a_{1}^{j} \frac{\partial}{\partial \omega^{j}}\right\lrcorner\left(d \omega^{i}-d \bar{\omega}^{i}\right)\right)\right\}_{i=1, \ldots, n}
$$

where the maximum is taken over all $v_{1}=\left(a_{1}^{1}, \ldots, a_{1}^{n}\right) \in \mathbb{R}^{n}$. The $k^{\text {th }}$ reduced Cartan character is then defined (as before) by

$$
\tilde{s}_{1}^{(1)}+\cdots+\tilde{s}_{k}^{(1)}=\max \operatorname{rank}\left\{\mathcal{C}\left(v_{1}\right) \cup \cdots \cup \mathcal{C}\left(v_{k}\right)\right\}
$$

where the maximum is taken over all $k$-tuples $v_{1}, \ldots, v_{k} \in \mathbb{R}^{n}$. Our "Gaussian 
elimination" of $d \omega^{i}-d \bar{\omega}^{i}$ in (5.56) resulted in the second equation in (5.57). This equation has the same structure as $d \omega^{i}$ does on $\mathcal{B}_{1}^{u}$ (after replacing $\alpha^{\kappa}$ by $\alpha^{\kappa}-\bar{\alpha}^{\kappa}$ ) modulo purely horizontal two-forms, see (5.54). Thus, since we project onto top order forms when computing reduced Cartan characters, the ones for our EDS, (5.58), agree with those of the partial moving frame $\mathcal{B}_{1}^{u}$ (cf. Section 5.5).

Let $E$ be an integral element satisfying (5.57). We claim that a basis, $\left\{\mathbf{V}_{1}, \ldots, \mathbf{V}_{n}\right\}$, for $E$ can always be chosen to be of the form

$$
\mathbf{V}_{i}=\frac{\partial}{\partial \omega^{i}}+\frac{\partial}{\partial \bar{\omega}^{i}}+w_{i}^{\kappa}\left(\frac{\partial}{\partial \alpha^{k}}+\frac{\partial}{\partial \bar{\alpha}^{\kappa}}\right)+z_{i}^{\kappa} \frac{\partial}{\partial \alpha^{\kappa}}
$$

The only non-trivial part of this claim is showing that such vectors are always tangent to $\mathcal{F}=\left\{\Sigma^{u}=\Sigma^{\bar{u}}\right\} \subset \mathcal{B}_{1}^{u} \times \mathcal{B}_{1}^{\bar{u}}$, but if $I^{u}$ and $I^{\bar{u}}$ are genuine invariants and components of the signatures $\Sigma^{u}$ and $\Sigma^{\bar{u}}$, then

$$
\mathbf{V}_{i}\left(I^{u}-I^{\bar{u}}\right)=\frac{\partial I^{u}}{\partial \omega^{i}}-\frac{\partial I^{\bar{u}}}{\partial \bar{\omega}^{i}}
$$

Moreover, $\frac{\partial I^{u}}{\partial \omega^{i}}$ and $\frac{\partial I^{\bar{u}}}{\partial \bar{\omega}^{i}}$ are also genuine invariants and must agree on $\mathcal{F}$ (since the signature is stabilized). Hence the expression (5.60) vanishes and the $\mathbf{V}_{i}$ are tangent to $\mathcal{F}$.

Next, let us compute the dimension of the space (in the appropriate Grassmannian) of integral elements satisfying (5.57). It turns out that this dimension agrees with the number of non-normalized group parameters of second order in $\mathcal{B}_{1,1}$. We can always take the basis of $E$ to be of the form (5.59). These basis vectors obviously satisfy the first and third equations in (5.57). Plugging a pair of basis vectors $\mathbf{V}_{a}$ and $\mathbf{V}_{b}$, of the form (5.59), into the second equation in 5.57) gives

$$
0=\left(A_{\kappa, j}^{i}\left(\alpha^{\kappa}-\bar{\alpha}^{\kappa}\right) \wedge \omega^{j}\right)\left\langle\mathbf{V}_{a}, \mathbf{V}_{b}\right\rangle=\left(A_{\kappa, j}^{i} \alpha^{\kappa} \wedge \omega^{j}\right)\left\langle\mathbf{v}_{a}, \mathbf{v}_{b}\right\rangle
$$


where

$$
\mathbf{v}_{i}=\frac{\partial}{\partial \omega^{i}}+z_{i}^{\kappa} \frac{\partial}{\partial \alpha^{\kappa}} .
$$

The dimension of the space of integral elements satisfying (5.57) is the number of free parameters $z_{i}^{\kappa}$ in the linear, homogeneous equations (5.61). By (5.54), these equations are the homogeneous part of the system

$$
d \omega^{i}\left\langle\mathbf{v}_{a}, \mathbf{v}_{b}\right\rangle=0
$$

on $\mathcal{B}_{1}^{u}$, and the two have the same solution space dimension. The structure equations $d \omega^{i}$ on $\mathcal{B}_{1}^{u}$ agree with those of $\mathcal{G}_{1}^{u v}$ (where $v$ is a section that intersects the cross-section) and recalling the discussion of integral elements in Remark 3.2.8. the dimension of the solution space to 5.62 is $r^{(2)}$, the number of non-normalized second order group parameters!

The Cartan-Kähler theorem for exterior differential systems with an independence condition, as in (5.47), says that an integral element for 5.47) can be integrated to an integral manifold $\mathcal{N}$ if the dimension of the solution space of 5.61, which we have shown to be $r^{(2)}$, and the reduced Cartan characters 5.58 satisfy Cartan's test:

$$
r^{(2)}=\sum i \tilde{s}_{i}^{(1)} .
$$

What is more, the integral manifold depends on $\tilde{s}_{i}^{(1)}$ free functions of $i$ variables. Since these quantities agree with their partial moving frame counterparts for $\mathcal{B}_{1}^{u}$, we have shown that this test is satisfied if and only if the partial moving frame $\mathcal{B}_{1}^{u}$ is involutive. Everything we have said about this first order problem trivially carries over to a higher order one (5.47). Summing up, we have the following.

Theorem 5.6.2. The graphs of two functions, $u$ and $\bar{u}$, whose jets land in the domain of definition of an involutive partial moving frame, $\mathcal{B}_{p}$, constructed by Algorithm 5.5.3, are congruent if and only if their signatures overlap. The general congruence map depends on $\tilde{s}_{i}^{(p)}$ functions of $i$ variables where $\tilde{s}_{i}^{(p)}$ are the reduced Cartan characters of $\mathcal{B}_{p}$. 
Combining this Theorem with Remark 5.5.11 we have the Lie-Tresse theorem for horizontal Lie pseudo-groups: The algebra of differential invariants is generated, under invariant differentiation, by a finite number of invariants 


\section{Chapter 6}

\section{Examples}

We now solve a few equivalence problems using Algorithm 5.5.3. But first a few words of guidance to the reader planning to apply this algorithm to a substantial equivalence problem.

Remark 6.0.1. The formulas for the lifted invariants $U_{J}^{\alpha}$ quickly get out of hand as $|J|$ increases, and directly solving a system of normalization equations, $U_{J}^{\alpha}=c_{J}^{\alpha}$, will overwhelm computer algebra systems in most examples for $|J| \geq 3$. How then did Cartan, and his disciples solve, with no help from machines, equivalence problems using his method? The key to effectively carry out the method is to take advantage of a few simplifying facts.

- By far the most important fact is that the recurrence formula only involves the linearization of lifted invariants at the identity. This provides immense simplification since we can obtain the form of the structure equations by computing

$$
\left.\frac{\partial U_{J}^{\alpha}}{\partial X_{K}^{i}}\right|_{\mathbb{1}} \mu_{K}^{i},
$$

which is very easy for computer algebra systems, even at high orders of $|J|$. These linearized expressions also tell us which group parameters can be normalized in which lifted invariant. 
- The fact that $U_{J}^{\alpha}$ is affine in the top order group parameters helps in solving for these and to compute the lower order part of $U_{J}^{\alpha}$ as in Example 5.6.1. What the original wielders of the equivalence method did was essentially breaking each $U_{J}^{\alpha}$ up into a top order part and lower order part and then solve a linear system for the top order parts, never explicitly writing out the complicated expressions for lifted invariants, see 5.52 .

- The structure equations of the invariant forms $\mu_{K}^{i}$ on partial moving frames combined with the recurrence formula can sometimes indicate which group parameter can be normalized in which lifted invariant $U_{J}^{\alpha}$. This means that we can, without explicitly solving any normalization equations, spy important information on the complicated normalization process.

Keeping these bullet points in mind, we now work out some examples.

\subsection{Equivalence of second order ordinary differ- ential equations}

Example 6.1.1. Consider the pseudo-group $\mathcal{G}$, acting on $\mathbb{R}^{3}$ with coordinates $\left(x, u, u_{x}\right)=(x, u, p)$, with defining equations

$$
X_{u}=X_{p}=U_{p}=0, \quad P=\frac{U_{x}+p U_{u}}{X_{x}}
$$

This is the fiber-preserving counterpart to the pseudo-group in Example 5.4.8. We spot the obvious integrability condition $P_{p}=U_{u} / X_{x}$ and the corresponding dependency $\mu_{p}^{p}=\mu_{u}^{u}-\mu_{x}^{x}$, and notice it is the only integrability condition appearing at the first stage, and adding it to $\mathcal{G}_{1}$ in fact makes $\mathcal{G}_{1}$ formally integrable. The other dependencies among the Maurer-Cartan forms on $\mathcal{G}_{1}$ are

$$
\mu_{u}^{x}=\mu_{p}^{x}=\mu_{p}^{u}=0, \quad \mu^{p}=\mu_{x}^{u}+P \mu_{u}^{u}-P \mu_{x}^{x}
$$


We extend the action of $\mathcal{G}$ on itself to the extra coordinate $u_{x x}=q$,

$$
q \mapsto Q=\frac{P_{x}+p P_{u}+q P_{p}}{X_{x}}=\frac{P_{x}+p P_{u}+q U_{u} / X_{x}}{X_{x}}
$$

and consider the equivalence problem for sections $q,(x, u, p) \mapsto(x, u, p, q(x, u, p))$, i.e. second order ordinary differential equations. We normalize all zero order lifted invariants, $X=U=P=Q=0$, resulting, first of all in

$$
\omega^{x}=-\mu^{x}, \quad \omega^{u}=-\mu^{y}, \quad \omega^{p}=-\mu^{p} .
$$

Also, equation 6.1 reduces to $\mu_{u}^{u}=\mu^{p}=-\omega^{p}$ and, by the recurrence formula, we have

$$
\begin{aligned}
0 & =d Q=Q_{X} \omega^{x}+Q_{U} \omega^{u}+Q_{P} \omega^{p}+\mu_{x}^{p}+P \mu_{u}^{p}+Q \mu_{u}^{u}-2 Q \mu_{x}^{x} \\
& =Q_{X} \omega^{x}+Q_{U} \omega^{u}+Q_{P} \omega^{p}+\mu_{x}^{p} .
\end{aligned}
$$

After normalizing $X=U=P=Q=0$ we are working on a partial moving frame $\mathcal{B}_{1}$ (as always we do not restrict to a specific section, working instead with the general jet coordinates $\left.j^{\infty} q_{\left.\right|_{x}}=\left(x, q, \ldots, q_{J}, \ldots\right)\right)$. Next we compute, on $\widetilde{\mathcal{B}}_{1}$, using all the linear dependencies among the $\mu$ from above,

$$
\begin{aligned}
& d \omega^{x}=-\mu_{x}^{x} \wedge \omega^{x}, \\
& d \omega^{u}=\omega^{x} \wedge \omega^{p}-\mu_{u}^{u} \wedge \omega^{u}, \\
& d \omega^{p}=\left(Q_{X} \omega^{x}+Q_{U} \omega^{u}+Q_{P} \omega^{p}\right) \wedge \omega^{x}-\mu_{u}^{p} \wedge \omega^{u}-\left(\mu_{u}^{u}-\mu_{x}^{x}\right) \wedge \omega^{p} .
\end{aligned}
$$

The horizontal parts we need to normalize are $Q_{U} \omega^{u} \wedge \omega^{x}$ and $Q_{P} \omega^{p} \wedge \omega^{x}$. The lifted invariant $Q_{X}$ disappears due to $\omega^{x} \wedge \omega^{x}=0$ and we can assume it is normalized to zero also; $Q_{X}$ depends on $P_{x x}$ which does not appear in any other lifted invariant and cannot contribute to any integral conditions/lower order invariants.

Since $d^{2}=0$, and $d \mu_{j}^{i}=\omega^{k} \wedge \mu_{j k}^{i}+\mu_{k}^{i} \wedge \mu_{j}^{k}$, we obtain, on $\widetilde{\mathcal{B}}_{1}$, by taking $d$ of 
both sides in the structure equation for $d \omega^{p}$,

$$
\begin{aligned}
0= & d Q_{U} \wedge \omega^{u} \wedge \omega^{x}+d Q_{P} \wedge \omega^{p} \wedge \omega^{x}+\left(\mu_{u x}^{p} \wedge \omega^{x}+\mu_{u u}^{p} \wedge \omega^{u}+\mu_{p p}^{p} \wedge \omega^{p}\right) \wedge \omega^{u} \\
& +\left(\mu_{u x}^{u} \wedge \omega^{x}+\mu_{u u}^{u} \wedge \omega^{u}\right) \wedge \omega^{p}-\mu_{x x}^{x} \wedge \omega^{x} \wedge \omega^{p}+\text { lower order terms }
\end{aligned}
$$

where "lower order terms" denotes terms involving Maurer-Cartan forms of order at most one. Comparing terms in the above equation, we see that

$$
\begin{aligned}
& d_{G} Q_{U}=-\mu_{x u}^{p}+\text { lower order terms } \\
& d_{G} Q_{P}=-\mu_{x x}^{x}+\mu_{u x}^{u}+\text { lower order terms. }
\end{aligned}
$$

This means that we can normalize $Q_{U}=Q_{P}=0$ and solve both equations for $P_{u x}$ and $X_{x x}$, respectively. The lower order terms from above are easily found (using a computer algebra system!) as follows. Since the lifted invariants $Q_{U}$ and $Q_{P}$ are affine in the second order group parameters, simply compute them directly and set any second order group parameter to zero. For $Q_{U}$ and $Q_{P}$, we find that the lower order terms are the group differentials, $d_{G}$, of

$$
-\frac{q_{p} P_{u}}{U_{u} X_{x}}-\frac{2 P_{u}^{2}}{U_{u}^{2}}+\frac{q_{u}}{X_{x}^{2}} \text { and } \frac{q_{p}}{X_{x}}+\frac{2 P_{u}}{U_{u}}
$$

respectively. We obtain (since $Q_{P}=Q_{u}=0$ on $\left.\widetilde{\mathcal{B}}_{1,1}\right)$,

$$
\begin{aligned}
& d_{G}\left(-\frac{q_{p} P_{u}}{U_{u} X_{x}}-\frac{2 P_{u}^{2}}{U_{u}^{2}}+\frac{q_{u}}{X_{x}^{2}}\right)=-Q_{P} \mu_{u}^{p}-2 Q_{U} \mu_{x}^{x}=0 \\
& d_{G}\left(\frac{q_{p}}{X_{x}}+\frac{2 P_{u}}{U_{u}}\right)=-Q_{P} \mu_{x}^{x}+2 \mu_{u}^{p}=2 \mu_{u}^{p}
\end{aligned}
$$

We have

$$
\begin{aligned}
& d_{G} Q_{U}=-\mu_{x u}^{p} \\
& d_{G} Q_{P}=-\mu_{x x}^{x}+\mu_{u x}^{u}+2 \mu_{u}^{p}
\end{aligned}
$$


By the defining equations for $\mathcal{G}$ we also have $\mu_{x u}^{u}=\mu_{u}^{p}$ and the recurrence formula now gives

$$
\begin{aligned}
& 0=d Q_{U}=-\mu_{x u}^{p}+Q_{U i} \omega^{i} \\
& 0=d Q_{P}=-\mu_{x x}^{x}+3 \mu_{u}^{p}+Q_{P i} \omega^{i}
\end{aligned}
$$

The second order group parameters that have not yet been normalized are $U_{u u}$ and $P_{u u}$, so we have $r^{(2)}=2$. The involutivity test for $\widetilde{\mathcal{B}}_{1,1}^{(1)}$ in part (b) of Algorithm 5.5.3 maximizes, over all $(a, b, c) \in \mathbb{R}^{3}$,

$$
\text { the rank of } \left.\left.\left\{\gamma_{1}\left(\left(a D_{X}+b D_{U}+c D_{P}\right)\right\lrcorner d \omega^{i}\right)\right|_{\widetilde{\mathcal{B}}_{1,1}^{(1)}}\right\}_{i=x, u, p} \text {. }
$$

The structure equations on $\widetilde{\mathcal{B}}_{1,1}^{(1)}$ are

$$
\begin{aligned}
& d \omega^{x}=-\mu_{x}^{x} \wedge \omega^{x}, \\
& d \omega^{u}=\omega^{x} \wedge \omega^{p}-\mu_{u}^{u} \wedge \omega^{u}, \\
& d \omega^{p}=-\mu_{u}^{p} \wedge \omega^{u}-\left(\mu_{u}^{u}-\mu_{x}^{x}\right) \wedge \omega^{p},
\end{aligned}
$$

and so maximizing $(6.3)$ is equivalent to maximizing the rank of the collection

$$
\left\{a \mu_{x}^{x}, b \mu_{u}^{u}, b \mu_{u}^{p}+c\left(\mu_{u}^{u}-\mu_{x}^{x}\right)\right\}
$$

which, again, is equivalent to maximizing the rank of the matrix

$$
\left[\begin{array}{ccc}
a & 0 & 0 \\
0 & b & 0 \\
-c & c & b
\end{array}\right]
$$

It is clear that this maximum rank is 3 , but $r^{(2)}=2<3$ and Cartan's test will fail. We then need to move up one order, analyzing $\mathcal{B}_{2}:=\mathcal{B}_{1,1}$ next.

We compute the exterior derivative of all non-normalized Maurer-Cartan forms 
of order 1 . Beginning with $\mu_{x}^{x}$, we have

$$
d \mu_{x}^{x}=\omega^{x} \wedge \mu_{x x}^{x}=\omega^{x} \wedge\left(3 \mu_{u}^{p}+Q_{P i} \omega^{i}\right) .
$$

Taking $d$ of both sides here and comparing terms gives

$$
\begin{aligned}
& d_{G} Q_{U P}=3 \mu_{u u}^{p}+\text { lower order terms } \\
& d_{G} Q_{P P}=3 \mu_{u p}^{p}+\text { lower order terms. }
\end{aligned}
$$

By the defining equations for $\mathcal{G}$ we know that $P_{u p}=U_{u u}\left(\right.$ and $\mu_{u p}^{p}=\mu_{u u}^{u}$ ) and so we can set $Q_{U P}=Q_{P P}=0$ solving for $P_{u u}$ and $U_{u u}$, respectively. (Note that at this point we have normalized all second order group parameters, see Remark 5.5.9). Once we know the top order dependencies of, say, $Q_{U P}$ we can obtain all of $d_{G} Q_{U P}$. The lower order terms in $d_{G} Q_{U P}$ are obtained by applying $d_{G}$ to

$$
\frac{q_{p u}}{U_{u} X_{x}}-\frac{P_{u} q_{p p}}{U_{u}^{2}}
$$

and those in $d_{G} Q_{P P}$ from

$$
\frac{q_{p p}}{U_{u}}
$$

Plugging all this information into the recurrence formula, we have,

$$
\begin{aligned}
& 0=d Q_{U P}=3 \mu_{u u}^{p}+Q_{U P i} \omega^{i} \\
& 0=d Q_{P P}=3 \mu_{u u}^{u}+Q_{P P i} \omega^{i} .
\end{aligned}
$$

We have normalized all second order group parameters and the equivalence problem is fully determinate of order at most 2. To obtain all the structure equations 
of our system we compute $d \mu_{u}^{u}$ and $d \mu_{u}^{p}$. Now,

$$
\begin{aligned}
d \mu_{u}^{u} & =\omega^{x} \wedge \mu_{u x}^{u}+\omega^{u} \wedge \mu_{u u}^{u} \\
& =\omega^{x} \wedge \mu_{u}^{p}-\omega^{u} \wedge\left(Q_{P P i} \omega^{i}\right) \\
& =\omega^{x} \wedge \mu_{u}^{p}-Q_{P P X} \omega^{u} \wedge \omega^{x}-Q_{P P P} \omega^{u} \wedge \omega^{p}
\end{aligned}
$$

and so, we have to compute $Q_{P P X}$ and $Q_{P P P}$ on $\mathcal{B}_{2,1}^{(1)}$ as these are invariants that must be preserved/normalized. Computer algebra systems are good at that sort of thing, and we find

$$
\begin{aligned}
& J_{1}:=Q_{P P X}=\frac{-q_{p u}+p q_{p p u}+q_{p p x}+q q_{p p p}}{U_{u} X_{x}} \\
& J_{2}:=Q_{P P P}=\frac{X_{x} q_{p p p}}{U_{u}^{2}}
\end{aligned}
$$

Notice that we can only normalize group parameters from $J_{1}$ on sections $j^{\infty} q$ where

$$
-q_{p u}+p q_{p p u}+q_{p p x}+q q_{p p p}
$$

is not zero. Similarly, we can only normalize group parameters from $J_{2}$ if $q_{p p p} \neq 0$. This is where our equivalence problem branches and we must partition $J^{\infty}(\mathcal{E})$ into subsets where different combinations of these lifted invariants can be normalized.

We still haven't computed $d \mu_{u}^{p}$, but we find that

$$
d \mu_{u}^{p}=\left(-Q_{U U}+\frac{1}{3} Q_{P U X}\right) \omega^{x} \wedge \omega^{u}+\mu_{u}^{p} \wedge \mu_{x}^{x}
$$

where

$$
-Q_{U U}+\frac{1}{3} Q_{P U X}=\frac{q_{p u x}+q_{u} q_{p p}-q_{p} q_{p u}+q q_{p p u}+p q_{p u u}-2 q_{u u}}{3 U_{u} X_{x}^{2}}-\frac{P_{u}}{3 U_{u}^{2} X_{x}} J_{1} .
$$

Say we are in the branch where $q_{p p p} \neq 0$ and we normalize $\frac{X_{x} q_{p p p}}{U_{u}^{2}}=1$ obtaining $X_{x}=q_{p p p} U_{u}^{2}$. At this point we have obtained an extra differential equation in $\mathcal{G}_{1}^{u v}$ 
and we need to go back to a first order partial moving frame $\mathcal{B}_{1}$ and compute its structure equations. All the different branches of this equivalence problem have been studied and documented, so we stop here and refer to [36] for more.

\subsection{Divergence equivalence of Lagrangians}

Example 6.2.1. Continuing Example 5.4.10, we extend the point-transformation $(x, u, p) \mapsto(X, U, P)$, where $P=\frac{U_{x}+p U_{u}}{X_{x}+p X_{u}}$, to a two dimensional space of variables representing $\tilde{E}(L)$ and $L_{p p}$. Call these variables $w$ and $z$, respectively. These variables transform according to (see Example 5.4.10)

$$
\begin{aligned}
& w \mapsto W=\frac{w}{P_{p}^{2} \cdot\left(p X_{u}+X_{x}\right)}, \\
& z \mapsto Z=\frac{z}{P_{p} \cdot\left(p X_{u}+X_{x}\right)}+\frac{p P_{u}+P_{x}}{p X_{u}+X_{x}} \cdot W .
\end{aligned}
$$

The defining equations of $\mathcal{G}$ in this case are

$$
X_{p}=U_{p}=0, \quad U_{x}=P\left(X_{x}+p X_{u}\right)-p U_{u}
$$

Differentiating the last equation w.r.t. $p$ gives the integrability condition

$$
U_{u}=P_{p} X_{x}+p P_{p} X_{u}+P X_{u}
$$

This makes $\mathcal{G}_{1}$ formally integrable. Prolonging these equations once and linearizing gives the following linear dependencies among the Maurer-Cartan forms, up to second order (after setting $X=U=P=0$ ).

$$
\begin{aligned}
& \mu_{p}^{x}=\mu_{p p}^{x}=\mu_{p u}^{x}=\mu_{p x}^{x}=\mu_{p}^{u}=\mu_{p p}^{u}=\mu_{p u}^{u}=\mu_{p x}^{u}=0, \\
& \mu_{x}^{u}=\omega^{p}, \quad \mu_{u}^{u}=\mu_{p}^{p}+\mu_{x}^{x}, \quad \mu_{p x}^{p}=\mu_{x x}^{x}+\mu_{u}^{p}, \quad \mu_{p p}^{p}=-2 \mu_{x}^{x} \\
& \mu_{u u}^{u}=\mu_{p u}^{p}, \quad \mu_{p p}^{p}=-2 \mu_{u}^{x}, \quad \mu_{x x}^{u}=\mu_{x}^{p}, \quad \mu_{u x}^{u}=\mu_{u}^{p} .
\end{aligned}
$$


In Example 5.4 .10 we normalized $W$ to 1 and $Z$ to 0 , and the recurrence formula gives, using (6.5), that

$$
\begin{aligned}
& 0=d W=W_{i} \omega^{i}-2 \mu_{p}^{p}+\mu_{x}^{x}, \\
& 0=d Z=Z_{i} \omega^{i}+\mu_{x}^{p} .
\end{aligned}
$$

Computing all $d \omega^{i}$ gives

$$
\begin{aligned}
& d \omega^{x}=\omega^{x} \wedge \mu_{x}^{x}+\omega^{u} \wedge \mu_{u}^{x}=\omega^{x} \wedge\left(2 \mu_{p}^{p}-W_{i} \omega^{i}\right)+\omega^{u} \wedge \mu_{u}^{x} \\
& d \omega^{u}=\omega^{x} \wedge \omega^{p}+3 \omega^{u} \wedge \mu_{p}^{p}, \\
& d \omega^{p}=\omega^{x} \wedge\left(-Z_{i} \omega^{i}\right)+\omega^{u} \wedge \mu_{u}^{p}+\omega^{p} \wedge \mu_{p}^{p} .
\end{aligned}
$$

Taking $d$ of both sides of the first equation and comparing the emerging threeforms involving second order Maurer-Cartan forms gives, modulo terms involving lower order Maurer-Cartan forms,

$$
d_{G} W_{U} \equiv-2 \mu_{p u}^{p}-\mu_{u x}^{x} .
$$

And computing the lower order dependencies of $d_{G} W_{U}$ shows that the entire recurrence formula is

$$
0=d W_{U}=d_{H} W_{U}+d_{G} W_{U}=W_{U i} \omega^{i}-W_{X} \mu_{u}^{x}-W_{P} \mu_{u}^{p}-2 \mu_{p u}^{p}-\mu_{u x}^{x} .
$$

We also learn from $d^{2} \omega^{x}=0$ that $W_{P}$ depends only on first order group parameters and we solve for $X_{u}$ in the equation $W_{P}=0$. We obtain

$$
X_{u}=-\frac{w_{p}}{3 P_{p}^{2}},
$$

and the recurrence formula is

$$
0=d W_{P}=W_{P i} \omega^{i}+3 \mu_{u}^{x} .
$$


This gives a new first order partial moving frame and we must start over, computing $d \omega^{i}$ again. Before doing that though, we finish our analysis of the equations (6.6). Turning to the third equation in $(6.6)$ we take $d$ of both sides and find that, modulo lower order Maurer-Cartan forms,

$$
d_{G} Z_{P} \equiv-\mu_{p x}^{p} \equiv-\mu_{x x}^{x}
$$

and including absorption, we have $0=d Z_{P}=Z_{P i} \omega^{i}-\mu_{x x}^{x}+\mu_{u}^{p}$. We solve for $X_{x x}$ in $Z_{P}=0$. We also discover that $d_{G} Z_{U} \equiv-\mu_{u x}^{p}$ and we can solve for $P_{u x}$ in $Z_{U}=0$. We also obtain the recurrence formula $0=d Z_{U}=Z_{U i} \omega^{i}-\mu_{u x}^{p}$.

Now we return to 6.6 with our new normalization of $X_{u}$ and find that

$$
\begin{aligned}
& d \omega^{x}=2 \omega^{x} \wedge \mu_{p}^{p}-\frac{1}{3} W_{P i} \omega^{u} \wedge \omega^{i}, \\
& d \omega^{u}=\omega^{x} \wedge \omega^{p}+3 \omega^{u} \wedge \mu_{p}^{p}, \\
& d \omega^{p}=\omega^{u} \wedge \mu_{u}^{p}+\omega^{p} \wedge \mu_{p}^{p} .
\end{aligned}
$$

Taking $d$ of both sides in the first equation, we learn that $d_{G} W_{P X} \equiv 6 \mu_{p u}^{p}$ and we can solve for $P_{p u}$ in $W_{P X}=0$, providing recurrence formula $0=d W_{P X}=$ $W_{P X i} \omega^{i}+6 \mu_{p u}^{p}+2 \mu_{u}^{p}$. We also discover that $W_{P P}$, on our normalized space, depends only on first order group parameters. We happen to be able to solve for $P_{p}$ in $W_{P P}=1$, to obtain

$$
P_{p}=\frac{\sqrt{3 w w_{p p}-4 w_{p}^{2}}}{\sqrt{3} w},
$$

if $\sqrt{3 w w_{p p}-4 w_{p}^{2}}$ is not zero. Recall that $w$ denoted $L_{p p}$ and so this means we can normalize $P_{p}$ if the following expression is nowhere zero,

$$
J:=\sqrt{3 L_{p p} L_{p p p p}-4 L_{p p p}^{2}} .
$$

For Lagrangians where this expression is identically zero, we cannot normalize $P_{p}$ 
and our equivalence problem branches. Assume for now that we can normalize $P_{p}$ at this stage. The recurrence formula is $0=d W_{P P}=W_{P P i} \omega^{i}-6 \mu_{p}^{p}$. This is another integrability condition and we must repeat the computation (6.7) using this normalization. We obtain

$$
\begin{aligned}
& d \omega^{x}=\frac{1}{3} W_{P P i} \omega^{x} \wedge \omega^{i}-\frac{1}{3} \omega^{u} \wedge \omega^{p}, \\
& d \omega^{u}=\omega^{x} \wedge \omega^{p}+\frac{1}{2} W_{P P i} \omega^{u} \wedge \omega^{i}, \\
& d \omega^{p}=\omega^{u} \wedge \mu_{u}^{p}+\frac{1}{6} W_{P P i} \omega^{p} \wedge \omega^{i} .
\end{aligned}
$$

Further calculations reveal that $W_{P P P}$ is the genuine invariant

$$
I=\frac{\left(-9 L_{p p}^{2} L_{5 p}+45 L_{p p} L_{p p p} L_{p p p p}-40 L_{p p p}^{3}\right)}{\sqrt{3} J^{3}} .
$$

We also observe from $d^{2} \omega^{x}=0$ that $W_{P P U}$ does not depend on any second order group parameters, so it is either a genuine invariant or it is possible to normalize $P_{u}$ in $W_{P P U}$. Computing the explicit dependence of $W_{P P U}$ on group parameters at the identity and applying all our previous normalizations gives

$$
3 d_{G} W_{P P U}=-\mu_{u}^{p}+W_{P X i} \omega^{i}-3 W_{U i} \omega^{i}
$$

and so we can normalize $P_{u}$ by setting $W_{P P U}=0$ to obtain

$$
\mu_{u}^{p}=\left(3 W_{P P U i}+W_{P X i}-3 W_{U i}\right) \omega^{i}
$$

At his point we have normalized all first order group parameters so the equivalence problem has been reduced to an equivalence problem for coframes on the base manifold. But recall that this holds only for Lagrangians where $J$ is nowhere 
zero. The final structure equations are

$$
\begin{aligned}
d \omega^{x} & =\frac{1}{3} I \omega^{x} \wedge \omega^{p}-\frac{1}{3} \omega^{u} \wedge \omega^{p} \\
d \omega^{u} & =\omega^{x} \wedge \omega^{p}+\frac{1}{2} W_{P P X} \omega^{u} \wedge \omega^{x}+\frac{1}{2} I \omega^{u} \wedge \omega^{p} \\
d \omega^{p} & =\omega^{u} \wedge\left(3 W_{P P U X}+W_{P X X}-3 W_{U X}\right) \omega^{x}+\omega^{u} \wedge\left(3 W_{P P U P}+W_{P P X}\right) \omega^{p} \\
& +\frac{1}{6} W_{P P X} \omega^{p} \wedge \omega^{x}
\end{aligned}
$$

The coefficients of the above structure equations are all genuine invariants. By Cartan's solution of equivalence problems for coframes, they generate the algebra of invariants for this problem under invariant differentiation with respect to the frame dual to $\left\{\omega^{x}, \omega^{u}, \omega^{p}\right\}$.

Remark 6.2.2. It is very interesting how this hybrid equivalence method provides combinations of lifted invariants, e.g.

$$
3 W_{P P U X}+W_{P X X}-3 W_{U X}
$$

that generate the algebra of differential invariants. Using the equivariant moving frame method alone, finding a generating set is a daunting task indeed.

\subsection{Medolaghi's pseudo-group}

Example 6.3.1. Consider the Lie pseudo-group of transformations

$$
X=f(x), \quad Y=f_{x}(x) y+g(x),
$$

with defining equations

$$
Y_{y}=X_{x}, \quad X_{y}=0
$$


extended to acting on the variable $u \in \mathbb{R}$ by

$$
u \mapsto U=u+\frac{Y_{x}}{X_{x}}
$$

As before, we are interested in the congruence problem of sections $(x, y) \mapsto$ $(x, y, u(x, y))$ under this pseudo-group. We begin by normalizing $X=Y=U=0$, and the recurrence formula gives

$$
0=d U=U_{X} \omega^{x}+U_{Y} \omega^{y}+\mu_{x}^{y}-\mu_{x}^{x}
$$

We normalize $Y_{x}$ from $U=0$ to obtain $Y_{x}=-u X_{x}$ and the zero-order structure equations become

$$
\begin{aligned}
& d \omega^{x}=\omega^{x} \wedge \mu_{x}^{x}, \\
& d \omega^{y}=\omega^{x} \wedge \mu_{x}^{y}+\omega^{y} \wedge \mu_{y}^{y}=\omega^{x} \wedge \mu_{x}^{x}-U_{Y} \omega^{y} \wedge \omega^{x}+\omega^{y} \wedge \mu_{x}^{x} .
\end{aligned}
$$

Taking the exterior derivative on both sides indicates that, modulo lower order Maurer-Cartan forms, $d_{G} U_{Y}=-\mu_{x x}^{x}$. We can therefore normalize $U_{Y}=0$ and solve for $X_{x x}$, sparing the reader the details, we obtain $X_{x x}=-u_{y} X_{x}$. The recurrence formula is $0=d U_{Y}=U_{Y i} \omega^{i}-\mu_{x x}^{x}$. The lifted invariant $U_{X}$ vanishes along with $\omega^{x} \wedge \omega^{x}$ in the structure equations, but this indicates that $Y_{x x}$ can be normalized in $U_{X}$. There are no non-normalized second order group parameters left so we have complete reduction on a first order partial moving frame $\mathcal{B}_{1}$. We have only one non-normalized group parameter of order one, and we compute

$$
d \mu_{x}^{x}=\omega^{x} \wedge \mu_{x x}^{x}=U_{Y Y} \omega^{x} \wedge \omega^{y} .
$$

One consequence of this formula is that $U_{Y Y}$ must depend on first order group parameters only. Indeed, on our restricted space, we have

$$
U_{Y Y}=\frac{u_{y y}}{X_{x}^{2}}
$$


Here is where our equivalence problem branches. If $u_{y y}<0$ we can normalize $U_{Y Y}=-1$, but if $u_{y y}=0$ we can not normalize $U_{Y Y}$ at all. Focusing on the third branch, where $u_{y y}>0$, we normalize $U_{Y Y}$ to 1 and obtain the deterministic coframe

$$
\begin{aligned}
\omega^{x} & =\sqrt{u_{y y}} d x, \\
\omega^{y} & =-u \sqrt{u_{y y}} d x+\sqrt{u_{y y}} d y .
\end{aligned}
$$

The only genuine invariants of this problem will appear as structure functions of this coframe on the base manifold and their coframe derivatives. The recurrence formula gives $0=d U_{Y Y}=U_{Y Y i} \omega^{i}-2 \mu_{x}^{x}$ and so

$$
\begin{aligned}
d \omega^{x} & =\frac{1}{2} U_{Y Y Y} \omega^{x} \wedge \omega^{y} \\
d \omega^{y} & =\frac{1}{2}\left(U_{Y Y Y}-U_{Y Y X}\right) \omega^{x} \wedge \omega^{y} .
\end{aligned}
$$

We can compute the invariants $U_{Y Y Y}$ and $U_{Y Y X}$ either directly from (6.11) or by first computing their lifted form and plugging in our normalizations. In any case, we have

$$
U_{Y Y Y}=\frac{u_{y y y}}{u_{y y}^{3 / 2}}, \quad U_{Y Y X}=\frac{u_{x y y}+u u_{y y y}+2 u_{y} u_{y y}}{u_{y y}^{3 / 2}} .
$$

Remark 6.3.2. Notice, again, that our methods have lead naturally to a generating system of invariants, namely $U_{Y Y Y}$ and $U_{Y Y X}$ restricted to our moving frame.

On sections that are affine in $y$, i.e. $u_{y y}=0$, we have $d \mu_{x}^{x}=0$ and the Poincarélemma guarantees that there exists a function $\alpha$ such that $d \alpha=\mu_{x}^{x}$. Using the definition of the unrestricted Maurer-Cartan form,

$$
\mu_{x}^{x}=\frac{1}{X_{x}}\left(d X_{x}-X_{x x} d x\right)
$$

we see that on our partial moving frame, where we normalized $X_{x x}=-u_{y} X_{x}$, we have $\mu_{x}^{x}=\frac{d X_{x}}{X_{x}}+u_{y} d x=d\left(\log \left(X_{x}\right)+x u_{y}\right)$ and the function $\alpha$ must have the 
form

$$
\alpha=\log \left(X_{x}\right)+\int u_{y} d x .
$$

The coframe can now be written

$$
\begin{aligned}
\omega^{x} & =\frac{e^{\alpha}}{e^{x u_{y}}} d x, \\
\omega^{y} & =-\frac{u e^{\alpha}}{e^{x u_{y}}} d x+\frac{e^{\alpha}}{e^{x u_{y}}} d y, \\
\sigma & =d \alpha,
\end{aligned}
$$

The structure functions in this case are

$$
\begin{aligned}
& d \omega^{x}=\omega^{x} \wedge \sigma, \\
& d \omega^{y}=\omega^{x} \wedge \sigma+\omega^{y} \wedge \sigma, \\
& d \sigma=0 .
\end{aligned}
$$

All the coefficients in these structure functions are constant so, in particular, the symmetry group of any affine section is a finite dimensional (local) Lie group with Lie algebra structure (6.14).

Remark 6.3.3. Notice that sections satisfying $u_{y y}=0$ do not make up a locally $\mathcal{G}$ invariant set $\mathcal{S}$ in $J^{\infty}(\mathcal{E})$. Further, the action of $\mathcal{G}^{\mathcal{S}}$ on $\mathcal{S}$ (cf. Definition 5.2.2) will never be free as $X_{x}$ cannot be normalized and therefore the equivariant moving frame is, on its own, not able to deduce the symmetry properties of sections $j^{\infty} u \in \mathcal{S}$. Since our formulation, via Theorem 5.3.4, characterizes $\mathcal{G}^{\mathcal{S}}$ by the collection of maps preserving restricted Maurer-Cartan forms these "singular" sections are placed on an entirely equal footing as regular jets and their analysis is no different. 


\subsection{Equivalence of differential operators}

Example 6.4.1. Consider a linear second order differential operator on $\mathbb{R}$,

$$
\mathscr{D}=f D^{2}+g D+h
$$

where $f, g, h: \mathbb{R} \rightarrow \mathbb{R}$ are real-analytic, and $f \neq 0$. When we apply $\mathscr{D}$ to a real-analytic function $u: \mathbb{R} \rightarrow \mathbb{R}$ we obtain the function

$$
f u^{\prime \prime}+g u^{\prime}+h u
$$

Now consider the pseudo-group, $\mathcal{G}$, of transformations of the $(x, u)$ of the form

$$
(x, u) \mapsto(\varphi(x), u \cdot \psi(x))=(X, U),
$$

where $\varphi, \psi: \mathbb{R} \rightarrow \mathbb{R}$ are real-analytic. Restricting to the set $\mathcal{X}=\{(x, u) \in$ $\left.\mathbb{R}^{2} \mid u>0\right\}$ and to $\psi>0$ this is a Lie pseudo-group of transformations of $\mathcal{E}$ that has defining equations

$$
X_{y}=0, \quad U_{u u}=0, \quad U_{u} u=U
$$

The elements of $\mathcal{G}$ preserve the space of linear operators and a transformation from $\mathcal{G}$ maps 6.15 to

$$
\overline{\mathscr{D}}=F \bar{D}^{2}+G \bar{D}+H
$$

where $\bar{D}$ is the derivative with respect to the transformed independent variables $X$ and the lifted coefficients $F, G, H$ have explicit formulas

$$
\begin{aligned}
& F=f \frac{X_{x}^{2}}{U_{u}}, \\
& G=-f \frac{2 U_{x} X_{x}-X_{x x} U_{u} u}{u U_{u}^{2}}+g \frac{X_{x}}{U_{u}}, \\
& H=-f \frac{U_{x x} U_{u} u-2 U_{x}^{2}}{u U_{u}^{2}}-g \frac{U_{x}}{u U_{u}^{2}}+\frac{h}{U_{u}} .
\end{aligned}
$$


Notice that since $\frac{U_{x}}{u}$ is independent of $u$, these lifted invariants are also independent of $u$ (as they should be be). Each operator $\mathscr{D}$ defines a section,

$$
(x, u) \mapsto(x, u, f(x), g(x), h(x)),
$$

in the trivial bundle $\mathcal{X} \times \mathbb{R}^{3} \rightarrow \mathcal{X}$ and two operators are equivalent if their respective sections are congruent under the extended action of $\mathcal{G}$ given by (6.16). Let us apply Algorithm 5.5.3 to this problem.

Notice that the extended action does depend on the second order group parameters and so we shall, initially, be working in $\widetilde{\mathcal{G}}_{2}$. We first normalize $X=0$, $U=1, F=1$ and $G=H=0$ and the recurrence formula gives

$$
\begin{aligned}
& 0=d F=F_{X} \omega^{x}+2 \mu_{x}^{x}-\mu_{u}^{u} \\
& 0=d G=G_{X} \omega^{x}-2 \mu_{x}^{x}-2 \mu_{x}^{u}+\mu_{x x}^{x} \\
& 0=d H=H_{X} \omega^{x}-\mu_{x x}^{u}
\end{aligned}
$$

This is equivalent to

$$
\begin{aligned}
\mu_{u}^{u} & =F_{X} \omega^{x}+2 \mu_{x}^{x} \\
\mu_{x x}^{x} & =-G_{X} \omega^{x}+2 \mu_{x}^{u} \\
\mu_{x x}^{u} & =H_{X} \omega^{x}
\end{aligned}
$$

Since $F$ depends only on first order group parameters we need to prolong it according to Remark 3.0 .3 . We find that on our partial moving frame

$$
\begin{aligned}
d F_{X} & =F_{X X} \omega^{x}-\mu_{x}^{u}+F_{X} \mu_{x}^{x}-F_{X} \mu_{u}^{u}+2 \mu_{x x}^{x} \\
& =F_{X X} \omega^{x}-\mu_{x}^{u}+F_{X} \mu_{x}^{x}-F_{X}\left(F_{X} \omega^{x}+2 \mu_{x}^{x}\right)+2\left(-G_{X} \omega^{x}+2 \mu_{x}^{u}\right) \\
& =\left(F_{X X}-2 G_{X}-F_{X}^{2}\right) \omega^{x}-F_{X} \mu_{x}^{x}+3 \mu_{x}^{u} .
\end{aligned}
$$


We can therefore normalize $F_{X}=0$ and solve for $U_{x}$. Explicitly,

$$
U_{x}=\frac{2}{3} g u X_{x}^{2}-\frac{1}{3} u f^{\prime} X_{x}^{2}=u \frac{2 g-f^{\prime}}{3} X_{x}^{2}
$$

On this smaller partial moving frame the above equation 6.17 becomes

$$
0=d F_{X}=\left(F_{X X}-2 G_{X}\right) \omega^{x}+3 \mu_{x}^{u} \Longleftrightarrow \mu_{x}^{u}=\frac{F_{X X}-2 G_{X}}{3} \omega^{x}
$$

The equation $F_{X}=0$ was still only first order so we must prolong it again. Since $3 \mu_{x}^{u}=\left(F_{X X}-2 G_{X}\right) \omega^{x}$ it is convenient to try to normalize $F_{X X}-2 G_{X}$, but on our partial moving frame we have

$$
F_{X X}-2 G_{X}=\frac{3 f f^{\prime \prime}+5 g f^{\prime}-6 f g^{\prime}+9 f h-2 f^{\prime 2}-2 g^{2}}{3 f^{2} X_{x}^{2}} .
$$

This lifted invariant is only normalizable if

$$
J:=3 f f^{\prime \prime}+5 g f^{\prime}-6 f g^{\prime}+9 f h-2 f^{\prime 2}-2 g^{2} \neq 0
$$

and so this equivalence problem branches at this juncture. If $J>0$ we can set $F_{X X}-2 G_{X}=1$ and solve for $X_{x}=\frac{\sqrt{J}}{\sqrt{3} f}$. In this branch we have normalized all first order group parameters and the equivalence problem becomes an equivalence problem the coframes on $\mathcal{X}$. The recurrence formula gives

$$
0=d\left(F_{X X}-2 G_{X}\right)=\left(F_{X X X}-2 G_{X X}\right) \omega^{x}-2 \mu_{x}^{x} \Longleftrightarrow \mu_{x}^{x}=\frac{F_{X X X}-2 G_{X X}}{2} \omega^{x}
$$

and the structure equations of the invariant coframe on $\mathcal{X}$ are

$$
\begin{aligned}
& d \omega^{x}=\omega^{x} \wedge \mu_{x}^{x}=0, \\
& d \omega^{u}=\omega^{x} \wedge \mu_{x}^{u}+\omega^{u} \wedge \mu_{u}^{u}=\left(F_{X X X}-2 G_{X X}\right) \omega^{u} \wedge \omega^{x} .
\end{aligned}
$$

To obtain the explicit expression for the genuine (once restricter to our partial moving frame) invariant $F_{X X X}-2 G_{X X}$ it is convenient to directly compute the 
exterior derivative $d \omega^{u}$. Setting

$$
a(x)=\frac{J}{3 f^{2}}
$$

the coframe $\omega^{x}, \omega^{u}$ has the explicit form

$$
\begin{aligned}
& \omega^{x}=X_{x} d x=\sqrt{a} d x \\
& \omega^{u}=U_{x} d x+U_{u} d u=u \frac{2 g-f^{\prime}}{3} a d x+f a d u .
\end{aligned}
$$

Now it is a relatively easy job to compute $d \omega^{u}$ and we obtain

$$
d \omega^{u}=\frac{3 f a^{\prime}+4 f^{\prime} a-2 g a}{3 f a^{5 / 2}} \omega^{x} \wedge \omega^{u}
$$

This invariant generates the algebra of differential invariants for this problem but these expressions were computed in [23] and the subsequent analysis was applied to decide which differential operators can be expressed as a bilinear combination of first order differential operators that generate a finite dimensional Lie algebra, 24].

In the more symmetric branch where $J$ is identically zero we have the invariant coframe $\omega^{x}, \omega^{u}, \mu_{x}^{x}$ on the fully determinate partial moving frame $\mathcal{B}_{2}$. The structure equations are

$$
\begin{aligned}
& d \omega^{x}=\omega^{x} \wedge \mu_{x}^{x} \\
& d \omega^{u}=2 \omega^{u} \wedge \mu_{x}^{x} \\
& d \mu_{x}^{x}=0
\end{aligned}
$$

and all differential operators with $J=0$ are equivalent and have a local symmetry Lie group with this structure. A normal form for the operators in this branch is the simple operator

$$
\mathscr{D}=D^{2}
$$




\section{Chapter 7}

\section{More general group actions}

So far we have studied pseudo-groups of transformations on a space $\mathcal{X}$ that could be extended to a trivial bundle $\mathcal{E} \rightarrow \mathcal{X}$. While this horizontal case already covers many interesting equivalence problems, it does not capture the extent of applications of Cartan's equivalence method. Most notably, we are missing the equivalence problem for partial differential equations. Therefore we now turn to a more general case of a Lie pseudo-group of transformations on a trivial bundle $\mathcal{E}$, where we drop the assumption of the action being horizontal, and ask when two (local) sections of $\mathcal{E}$ are congruent. So, let $\mathcal{G}$ be a Lie pseudo-group of transformations on $\mathcal{E}$, where, for now, we make no assumptions on the form of the $\varphi$ from $\mathcal{G}$. Since all our considerations are local, we, as always, focus on sections of $\mathcal{E}$ that are locally the graphs of functions from $\mathcal{X}$ to $\mathcal{U}$. Letting $u(x)$ and $\bar{u}(\bar{x})$ be such functions, a transformation $\varphi \in \mathcal{G}$ maps (the graph of) $u$ to $\bar{u}$ if

$$
\bar{u}^{\alpha}\left(X_{\left.\right|_{u}}\right)=\left.U^{\alpha}\right|_{u} .
$$

Notice that we also need to restrict the target variable $X$ to $u$ as $X$ may very well be a function of $u$ in this more general setting. The set of transformations that satisfy the determining equations for $\mathcal{G}$ and the equations (7.1) are the equivalence maps for $u$ and $\bar{u}$. But, and here is the difference between this case and the 
horizontal one, the equations (7.1) do not define a system of differential equations on $\mathcal{E}$. Rather, they describe the behavior of $\varphi$ along the graph of $u$ only, and so we have a kind of initial value problem. How do we then proceed with this equivalence problem? To warm up for the main discussion, let us first study the simpler case of a finite dimensional Lie group. The congruence problem of sections of $\mathcal{E}$ under the action of a Lie group on the ambient space has (when the action is effective) a complete solution and is, as it turns out, identical to the Lie pseudo-group case for actions that are eventually free (see [44]).

\subsection{Congruence in a Lie group, a groupoid ap- proach}

Recall Theorem 5.3.4, which was the basis on which we built our solution to the equivalence problem in earlier sections. The Lie group analog of that result is as follows.

Theorem 7.1.1. Two submanifolds in a Lie group, $S \stackrel{\iota}{\hookrightarrow} G$ and $\bar{S} \stackrel{\bar{\iota}}{\hookrightarrow} G$, are (locally) congruent under the right action of a group element $g \in G, S \cdot g=\bar{S}$, if and only if there is a (local) transformation $f: S \rightarrow \bar{S}$ such that

$$
f^{*} \iota^{*} \mu=\iota^{*} \mu
$$

for each right-invariant Maurer-Cartan form $\mu$ on $G$.

Every Lie group is locally a Lie pseudo-group. To see why, let $\mathcal{X} \subset G$ be a local coordinate chart of a Lie group $G$ that we identify with an open neighborhood of $0 \in \mathbb{R}^{n}$. For a Lie group element $g \in G$, that is close enough to the identity to be a diffeomorphism between two open subsets of $\mathcal{X}$, we denote by $\varphi_{g}$ the right action of $g$ on $\mathcal{X}, x \mapsto x \cdot g$. The Lie group obviously acts freely on $\mathcal{X}$, and we assume that it acts real-analytically (this is actually no restriction, see [35]). The Lie group also acts freely on each diffeomorphism groupoid $\mathcal{D}_{p}(\mathcal{X}), 0 \leq p \leq \infty$, 
by the right action

$$
g \cdot j^{p} \psi_{\left.\right|_{x}}=\left.j^{p} \psi_{\left.\right|_{x}} \cdot j^{p} \varphi_{g}^{-1}\right|_{\varphi_{g}(x)},
$$

where on the right hand side we have the groupoid operation on $\mathcal{D}_{p}$. We define the sets

$$
G_{\mathcal{X}}^{x}:=\left\{g \in G \mid \varphi_{g}(x) \in \mathcal{X}\right\}
$$

for each $x \in \mathcal{X}$, i.e. $G_{\mathcal{X}}^{x}$ are the group elements that map $x$ into $\mathcal{X}$. Each set $G_{\mathcal{X}}^{x}$ is an open neighborhood of the identity in $G$. The subset of $\mathcal{D}_{\infty}$, given by

$$
\mathcal{G}_{\infty}:=\bigcup_{x \in \mathcal{X}} \bigcup_{g \in G_{\mathcal{X}}^{x}} j^{\infty} \varphi_{\left.g\right|_{x}}
$$

is a differential system in $J^{\infty}(\mathcal{X} \times \mathcal{X})$ that determines a Lie pseudo-group $\mathcal{G}$. Notice that since each $\varphi_{g}$ is real analytic, the map $g \mapsto j^{\infty} \varphi_{\left.g\right|_{x}}$ for $g \in G_{\mathcal{X}}^{x}$ is one-to-one and thus a diffeomorphism from $G_{\mathcal{X}}^{x}$ into a vertical fiber in $\mathcal{G}_{\infty}$. The vertical fibers in $\mathcal{G}_{\infty}$ then have the same dimension as the base space $\mathcal{X}$. This means that the determining equations of $\mathcal{G}$ are first order and maximally overdetermined (this is not surprising since group elements in a neighborhood of the identity in $G$ can be obtained by integrating (exponentiating) tangent vectors at the identity). We then have that $\mathcal{G}_{0}$ and $\mathcal{G}_{\infty}$ are diffeomorphic.

Since the right action 7.2 of $G$ on $\mathcal{G}_{\infty}$ preserves the target coordinates, the space $\mathcal{G}_{\infty}$ is partitioned into orbits; the $\tau$-vertical fibers $\mathcal{G}_{\infty}{ }^{1}$. Similarly, in the diffeomorphism groupoid $\mathcal{D}_{\infty}(\mathcal{X})$, the right action of a local diffeomorphism $\psi$ preserves $\tau$-vertical fibers, and the Maurer-Cartan forms restricted to these fibers provide a right-invariant coframe on each fiber. Hence, restricting the right invariant Maurer-Cartan forms of $\mathcal{D}_{\infty}$ to a $\tau$-vertical fiber $\mathcal{G}_{\infty}{ }^{X} \cong \mathcal{G}_{0}{ }^{X} \cong \mathcal{X}$ (where $\cong$ indicates a diffeomorphism) will give a right-invariant coframe on $\mathcal{X}$, namely the (restricted) lifted horizontal coframe, $\omega=\left\{\omega^{1}, \ldots, \omega^{n}\right\}$. Since the right action of $G$ on $\mathcal{X}$ agrees with that on $\mathcal{G}_{0}{ }^{X}$, the horizontal coframe $\omega$ is a basis for the Maurer-Cartan form on $\mathcal{G}$ in the neighborhood $\mathcal{X}$.

Remark 7.1.2. Similarly, let $G$ be an $n$-dimensional Lie group that acts effectively 
(and real-analytically) on an open set $\mathcal{X} \subset \mathbb{R}^{n}$, i.e. $x \cdot g=x, \forall x \in \mathcal{X}$ means that $g$ is the identity. Then, writing $\varphi_{g}$ for the transformation $x \mapsto x \cdot g$, the map $g \mapsto j^{\infty} \varphi_{\left.g\right|_{x}}$ is one-to-one (by effectiveness) from the open neighborhood $G_{\mathcal{X}}^{x} \subset G$ to $\mathcal{D}_{\left.\infty\right|_{x}}$. We then have a real-analytic differential system as in (7.3) that determines a Lie pseudo-group $\mathcal{G}$. Since each vertical fiber in $\mathcal{G}_{\infty}$ is finite dimensional, this system is maximally over-determined at some order $p+1$ and $\mathcal{G}_{\infty} \cong \mathcal{G}_{p}$. Notice that this means that all reduced Cartan characters of order $p+1$ (and above) are zero and these equations are regular in the sense of Definition 3.0.6. Each $\tau$-vertical fiber $\mathcal{G}_{p}{ }^{X}$ is locally homeomorphic to $G$ and restricting the Maurer-Cartan forms of $\mathcal{D}_{\infty}$ to $\mathcal{G}_{p}{ }^{X}$ gives an invariant coframe that has the same structure equations as the Maurer-Cartan forms on $G$. Corollary 5.3.5 now guarantees that the maps $\Lambda: \mathcal{G}_{p}{ }^{X} \rightarrow \mathcal{G}_{p}{ }^{X}$ that preserve this coframe are precisely the elements of $G$, or, rather, their right actions.

Example 7.1.3. To illustrate the previous remark, consider the projective group $P G L(2, \mathbb{R})$ acting on the projective line $P^{1} \mathbb{R}$. In coordinates the action is via linear fractional transformations,

$$
x \mapsto g \cdot x=X=\frac{\alpha x+\beta}{\gamma x+\delta} .
$$

The defining equations for the maps $X$ are found by experiment/implicitization, but $X$ must satisfy the well known Schwarzian equation

$$
X_{x x x} X_{x}-\frac{3}{2} X_{x x}=0
$$

The linearization at the identity of this equation, satisfied by all Lie algebroid elements $\zeta(x) \frac{\partial}{\partial x}$, is simply

$$
\zeta_{x x x}=0 .
$$

Therefore a basis for the Maurer-Cartan forms restricted to the $\tau$-vertical fiber $\mathcal{G}^{0}$ is given by the forms

$$
\omega^{x}, \quad \mu_{x}^{x}, \quad \mu_{x x}^{x} .
$$


Plugging all these restrictions into the structure equations for the diffeomorphism groupoid $\mathcal{D}_{\infty}(\mathbb{R})$ gives the structure equations on any $\tau$-vertical fiber of $\mathcal{G}$,

$$
\begin{gathered}
d \omega^{x}=\mu_{x}^{x} \wedge \omega^{x} \\
d \mu_{x}^{x}=\omega^{x} \wedge \mu_{x x}^{x} \\
d \mu_{x x}^{x}=-\mu_{x}^{x} \wedge \mu_{x x}^{x}
\end{gathered}
$$

which agrees with the structure equations of the Maurer-Cartan forms on $P G L(2, \mathbb{R})$.

Next, we need a trivial modification of Theorem 5.3.4 that will be the basis for the rest of our discussion and of the generalization of our methods (to be presented elsewhere). It has the same exact proof as Theorem 5.3.4.

Theorem 7.1.4. Let $\mathcal{G}$ be a Lie pseudo-group of transformations on the base manifold $\mathcal{X}$, and let $S$ and $\bar{S}$ be submanifolds of $\mathcal{X}$. Let $\mathcal{G}_{p}$ be formally integrable and let $s: S \rightarrow \mathcal{G}_{p}{ }^{X_{0}}$ and $\bar{s}: \bar{S} \rightarrow \mathcal{G}_{p}{ }^{X_{0}}$ be two functions into the $\tau$-vertical fiber $\mathcal{G}_{p}{ }^{X_{0}}$. A local transformation $f: S \rightarrow \bar{S}$ preserves all the pulled-back MaurerCartan forms,

$$
f^{*} \bar{s}^{*} \mu_{K}^{i}=s^{*} \mu_{K}^{i}, \quad|K|<p,
$$

if and only if there is a local section, $f_{p}$ of $\mathcal{G}_{p} \rightarrow \mathcal{X}$ that satisfies the following.

(a) The diagram

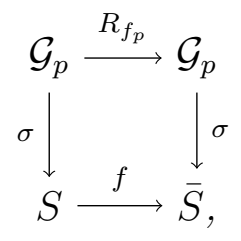

commutes, which, roughly, means that $R_{f_{p}}$ agrees with $f$ on the base manifold.

(b)

$$
\left(f_{p}\right)^{*} \mu_{K}^{i}=0, \quad|K|<p .
$$


(c) $R_{f_{p}}$ takes the graph of $s$ to the graph of $\bar{s}$.

Remark 7.1.5. The section $f_{p}$ annihilates the contact co-distribution on $\mathcal{G}_{p}$, which is an obvious necessary condition for there to exist an extension of $f_{p}$ to a graph of a local diffeomorphism in $\mathcal{G}$.

We now present a solution of the congruence problem of submanifolds under an effective Lie group action based on Theorem 7.1.4.

Theorem 7.1.6. Let $G$ be a Lie group acting effectively on a manifold $\mathcal{X}$, and let the associated differential system, $\mathcal{G}_{\infty}$, be maximally over-determined at order $p+1$ as in Remark 7.1.2. Given two submanifolds, $S, \bar{S} \subset \mathcal{X}$ and a map $f: S \rightarrow \bar{S}$, then $f$ is the restriction of an element $\varphi_{g}, g \in G$, if and only if there are two functions $s: S \rightarrow \mathcal{G}_{p+1}{ }^{X_{0}}$ and $\bar{s}: \bar{S} \rightarrow \mathcal{G}_{p+1}{ }^{X_{0}}$, for some $X_{0} \in \mathcal{X}$, such that

$$
f^{*} \bar{s}^{*} \mu_{K}^{i}=s^{*} \mu_{K}^{i}, \quad|K|<p+1
$$

Proof. Assume that $g \in G$ maps $S$ to $\bar{S}$ and let $s: S \rightarrow \mathcal{G}_{p+1}$ be any section. Then $\bar{s}:=R_{\varphi_{g}} \cdot s$ is the graph of a well-defined function on $\bar{S}$ (which we identify with $\bar{s}$ itself) and $\varphi_{g}$ obviously preserves the Maurer-Cartan forms of $\mathcal{G}$ once pulledback by $s$ and $\bar{s}$. To prove the converse, let $S, \bar{S} \subset \mathcal{X}$ be two submanifolds of $\mathcal{X}$, $s:\left.S \rightarrow \mathcal{G}_{p+1}\right|^{X_{0}}$ and $\bar{s}: \bar{S} \rightarrow \mathcal{G}_{p+1}{ }^{X_{0}}$ and let $f: S \rightarrow \bar{S}$ be a local transformation that satisfies $(7.5)$. The differential system $\mathcal{G}_{\infty}$ is maximally over-determined at order $p+1$ and so $\mathcal{G}_{\infty} \cong \mathcal{G}_{p+1} \cong \mathcal{G}_{p}$. By Remark 7.1.2 restricting the MaurerCartan forms $\mu_{K}^{i},|K|<p+1$ to a $\tau$-vertical fiber $\mathcal{G}_{p+1}{ }^{X_{0}}$ will give a coframe with the same structure equations as $G$. By Theorem 7.1.4 a section $f_{p+1}$ of $\mathcal{G}_{p+1}$ exists such that $f_{p+1}$ annihilates the contact forms on $\mathcal{G}_{p+1}$ and $R_{f_{p+1}}$ takes the graph of $s$ to the graph of $\bar{s}$, and such that (7.4) commutes. We wish to extend (or integrate) $f_{p+1}$ to an element of $G$, and to do that we write this problem as an exterior differential system.

Consider the product space $\mathcal{G}_{p+1}{ }^{X_{0}} \times \mathcal{G}_{p+1}{ }^{X_{0}}$ and the submanifold thereof 
parametrized by $S$ such that

$$
\mathcal{N}:=\left\{\left.(x, s(x), f(x), \bar{s}(f(x))\} \subset \mathcal{G}_{p+1}\right|^{X_{0}} \times\left.\mathcal{G}_{p+1}\right|^{X_{0}} .\right.
$$

For the projections onto the first and second coordinates in $\mathcal{G}_{p+1}{ }^{X_{0}} \times \mathcal{G}_{p+1}{ }^{X_{0}}$, denoted $\gamma_{1}$ and $\gamma_{2}$, we denote the pulled-back Maurer-Cartan forms by $\mu_{K}^{i}=\gamma_{1}^{*} \mu_{K}^{i}$ and $\bar{\mu}_{K}^{i}=\gamma_{2}^{*} \mu_{K}^{i}$. As is easily seen, the condition 7.5 is equivalent to

$$
\left(\mu_{K}^{i}-\bar{\mu}_{K}^{i}\right)_{\left.\right|_{\mathcal{N}}}=0, \quad|K|<p+1
$$

Since the structure equations of the $\mu_{K}^{i}$ on $\mathcal{G}_{p+1}{ }^{X_{0}}$ are the same as those for the Maurer-Cartan forms on $G$, and, in particular, the structure functions are all constant, the $\mu_{K}^{i}-\bar{\mu}_{K}^{i}$ form an involutive system in the sense of the Frobenius theorem:

$$
\begin{aligned}
d\left(\mu_{K}^{i}-\bar{\mu}_{K}^{i}\right) & =c_{K, j, k}^{i, L, M} \mu_{L}^{j} \wedge \mu_{M}^{k}-c_{K, j, k}^{i, L, M} \bar{\mu}_{L}^{j} \wedge \bar{\mu}_{M}^{k} \\
& =c_{K, j, k}^{i, L, M} \mu_{L}^{j} \wedge\left(\mu_{M}^{k}-\bar{\mu}_{M}^{k}\right)+c_{K, j, k}^{i, L, M}\left(\mu_{L}^{j}-\bar{\mu}_{L}^{j}\right) \wedge \bar{\mu}_{M}^{k} .
\end{aligned}
$$

By the uniqueness of integral manifolds in the Frobenius theorem we can integrate $\mathcal{N}$, uniquely, to an integral manifold, $\mathcal{M}$, of dimension $k=\operatorname{dim} G=\operatorname{dim} \mathcal{G}_{p+1}{ }^{X_{0}} \geq$ $\operatorname{dim} S$ (here we actually have to assume that $k \geq \operatorname{dim} S$ but this is always the case). Since $\omega^{i}-\bar{\omega}^{i}=\mu^{i}-\bar{\mu}^{i}$ vanishes on this integral manifold, we deduce that we may parametrize $\mathcal{M}$ according to

$$
(x, t) \mapsto(x, H(x, t), \bar{f}(x), \bar{H}(x, t))
$$

where $x$ are in an open neighborhood of $S, t \in \mathbb{R}^{k-n}$ are some parameters, and $\bar{f}$ is a diffeomorphism that agrees with $f$ on $S$. Since the parameter $t$ only affects the choice of sections $s$ and $\bar{s}$, we may set these to an arbitrary value, and $\bar{f}$ is our unique extension of $f$ that preserves the Maurer-Cartan forms $\mu_{K}^{i}$ once pulled back by the extensions on $s$ and $\bar{s}$. By Theorem 5.3.4, $\bar{f} \in \mathcal{G}$. 
Remark 7.1.7. The most important aspect of this finite dimensional case was the fact that we could invoke the Frobenius theorem to uniquely integrate the submanifold supplied by Theorem 7.1.4. While this method will not be available in general for Lie pseudo-groups, some important special cases do allow for the integration of the maps provided by Theorem 7.1.4. See the remarks at the end of this section.

Remark 7.1.8 (An easy proof of the congruence theorem in Lie groups). We just mention that the "technique of the graph" we have used many times now, where we construct the graph of our desired solutions as integral manifolds provides a trivial proof of Theorem 7.1.1. Let $S$ and $\bar{S}$ be submanifolds of equal dimension in a Lie group $G$. Let $\omega^{1}, \ldots, \omega^{n}$ be a basis for the Maurer-Cartan forms on $G$ and let $f: S \rightarrow \bar{S}$ be a map that preserves the $\omega^{i}$. Then $f$ is the restriction of a group element. To see why, consider the product $G \times G$, and the projections onto the first and second coordinates, $\gamma_{1}$ and $\gamma_{2}$.

Denote by $\omega^{i}$ the pull-back $\gamma_{1}^{*} \omega^{i}$ and by $\bar{\omega}^{i}$ the $\gamma_{2}^{*} \omega^{i}$. Consider the submanifold, $\mathcal{N}$, of $G$ parametrized by $S$,

$$
x \mapsto(x, f(x))
$$

Then, the fact that $f$ preserves the $\omega^{i}$ is equivalent to

$$
\left.\left(\omega^{i}-\bar{\omega}^{i}\right)\right|_{\mathcal{N}}=0
$$

Now, similar calculations as in (7.6) show that the differential system generated by $\omega^{i}-\bar{\omega}^{i}$ is involutive in the sense of the Frobenius theorem. This guarantees that $\mathcal{N}$ can be uniquely integrated to an $n$ dimensional submanifold of $G \times G$. It is easy to see that this larger submanifold is the graph of a local diffeomorphism that pulls $\bar{\omega}^{i}$ back to $\omega^{i}$. Since group elements do the same thing, the uniqueness part of Frobenius implies that this diffeomorphism must agree with some $g \in G$.

The central issue for the development of a more general equivalence method than for horizontal actions is in deciding for what kind of Lie pseudo-groups a 
section $f_{p}$ provided by Theorem 7.1.4 can be "integrated" to the graph of a bonafide pseudo-group element. Note that since $f_{p}$ annihilates contact forms there is some hope that it can be integrated in this fashion. The solution to these issues appears elsewhere, but we should mention that it includes Lie pseudo-groups that eventually become free (see [44]) and all Lie pseudo-groups acting on jet spaces by prolongation. The second case therefore covers all symmetry and equivalence analysis of differential equations. 


\section{Bibliography}

[1] I. Berchenko and P. J. Olver. Symmetries of polynomials. Journal of Symbolic Computation, 29(4-5):485-514, 2000.

[2] M. Boutin. Numerically invariant signature curves. International Journal of Computer Vision, 40(3):235-248, 2000.

[3] R. L. Bryant. On notions of equivalence of variational problems with one independent variable. Contemp. Math, 68:65-76, 1987.

[4] R. L. Bryant and P. A. Griffiths. Characteristic cohomology of differential systems (I): General theory. Journal of the American Mathematical Society, 8(3):507-596, 1995.

[5] E. Calabi, P. J. Olver, C. Shakiban, A. Tannenbaum, and S. Haker. Differential and numerically invariant signature curves applied to object recognition. International Journal of Computer Vision, 26(2):107-135, 1998.

[6] E. Calabi, P. J. Olver, and A. Tannenbaum. Affine geometry, curve flows, and invariant numerical approximations. Advances in Mathematics, 124(1):154196, 1996.

[7] É. Cartan. Les sous-groupes des groupes continus de transformations. In Oeuvres-Complètes, Part II, volume 2, pages 719-856. Gauthier-Villars, Paris, 1953. 
[8] É. Cartan and J. Leray. La théorie des groupes finis et continus et la géométrie différentielle: traitées par la méthode du repère mobile. 1937.

[9] S.-S. Chern. On the curvatura integra in a Riemannian manifold. Annals of Mathematics, pages 674-684, 1945.

[10] L.E. Dixon. Finiteness of the odd perfect and primitive abundant numbers with $n$ distinct prime factors. American Journal of Mathematics, 35:413-422, 1913.

[11] C. Ehresmann. Introduction à la théorie des structures infinitésimales et des pseudo-groupes de Lie. Géometrie Différentielle, pages 97-110, 1953.

[12] M. Fels and P. J. Olver. Moving coframes: II. Regularization and theoretical foundations. Acta Applicandae Mathematica, 55(2):127-208, 1999.

[13] R. B. Gardner. Differential geometric methods interfacing control theory. Birkhauser, Boston, 1983.

[14] R. B. Gardner. The method of equivalence and its applications. SIAM, 1989.

[15] R. B. Gardner and W. F. Shadwick. Equivalence of one dimensional Lagrangian field theories in the plane I. In Global Differential Geometry and Global Analysis 1984, pages 154-179. Springer, 1985.

[16] V. P. Gerdt. On the relation between Pommaret and Janet bases. In V. G. Ganzha, E. W. Mayr, and E. V. Vorozhtsov, editors, Computer Algebra in Scientific Computing: CASC 2000, pages 167-181. Springer Berlin Heidelberg, Berlin, Heidelberg, 2000.

[17] V.P. Gerdt and Y.A. Blinkov. Involutive bases for polynomial ideals. Math. Comput. Simul., 45(5-6):519-541, 1998.

[18] P. A. Griffiths. On Cartan's method of Lie groups and moving frames as applied to uniqueness and existence questions in differential geometry. Duke Math. J, 41(4):775-814, 1974. 
[19] L. Hsu, N. Kamran, and P. J. Olver. Equivalence of higher-order Lagrangians. II. The Cartan form for particle Lagrangians. Journal of mathematical physics, 30(4):902-906, 1989.

[20] E. Hubert. Differential invariants of a Lie group action: syzygies on a generating set. Journal of Symbolic Computation, 44(4):382-416, 2009.

[21] M. Janet. Sur les systemes d'equation aux derivees partielles. J Math Pure Appl, 3:65-151, 1920.

[22] N. Kamran. Contributions to the study of the equivalence problem of Élie Cartan and its applications to partial and ordinary differential equations, volume 45. Acad. Royale de Belgique, 1989.

[23] N. Kamran and P. J. Olver. Equivalence of differential operators. SIAM journal on mathematical analysis, 20(5):1172-1185, 1989.

[24] N. Kamran and P. J. Olver. Lie algebras of differential operators and Liealgebraic potentials. Journal of Mathematical Analysis and Applications, 145(2):342-356, 1990.

[25] N. Kamran and P. J. Olver. Equivalence of higher order Lagrangians. I. Formulation and reduction. J. Math. Pures et Appliquees, 70:369-391, 1991.

[26] N Kamran and W.F. Shadwick. A differential geometric characterization of the first Painlevé transcendent. Mathematische Annalen, 279(1):117-123, 1987.

[27] J. P. Kenney. Evolution of differential invariant signatures and applications to shape recognition. $\mathrm{PhD}$ thesis, University of Minnesota, 2009.

[28] K. Kodaira and D. C. Spencer. On deformations of complex analytic structures, I. Annals of Mathematics, pages 328-401, 1958. 
[29] K. Kodaira and D. C. Spencer. On deformations of complex analytic structures, II. Annals of Mathematics, pages 403-466, 1958.

[30] K. Kodaira and D. C. Spencer. On deformations of complex analytic structures, III. Stability theorems for complex structures. Annals of Mathematics, pages 43-76, 1960.

[31] I. A. Kogan and P. J. Olver. Invariant Euler-Lagrange equations and the invariant variational bicomplex. Acta Applicandae Mathematica, 76(2):137$193,2003$.

[32] A. Kumpera and D. C. Spencer. Lie Equations, volume 1. Princeton University Press, 1972.

[33] M. Kuranishi. On É. Cartan's prolongation theorem of exterior differential systems. American Journal of Mathematics, 79(1):1-47, 1957.

[34] P. Medolaghi. Classificazione delle equiazioni alle derivative parziali del secondo ordine, che ammettono un gruppo infinito di transformazioni puntuali. Ann. Mat. Pura Appl., 1(3):229-263, 1898.

[35] P. W. Michor. Topics in differential geometry, volume 93. American Mathematical Soc., 2008.

[36] P. J. Olver. Equivalence, invariants and symmetry. Cambridge University Press, 1995.

[37] P. J. Olver. Classical invariant theory, volume 44. Cambridge University Press, 1999.

[38] P. J. Olver. Applications of Lie groups to differential equations, volume 107. Springer Science \& Business Media, 2000.

[39] P. J. Olver. Geometric foundations of numerical algorithms and symmetry. Applicable Algebra in Engineering, Communication and Computing, 11(5):417-436, 2001. 
[40] P. J. Olver. Generating differential invariants. Journal of Mathematical Analysis and Applications, 333(1):450-471, 2007.

[41] P. J. Olver. Invariant submanifold flows. Journal of Physics A: Mathematical and Theoretical, 41(34):344017, 2008.

[42] P. J. Olver and J. Pohjanpelto. Maurer-Cartan forms and the structure of Lie pseudo-groups. Selecta Mathematica, New Series, 11(1):99-126, 2005.

[43] P. J. Olver and J. Pohjanpelto. Moving frames for Lie pseudo-groups. Canadian J. Math, 60(6):1336-1386, 2008.

[44] P. J. Olver and J. Pohjanpelto. Differential invariant algebras of Lie pseudogroups. Advances in Mathematics, 222(5):1746-1792, 2009.

[45] C. Riquier. Sur une question fondamentale du calcul integrale. Acta Math, 23:203-332, 1900.

[46] W. Seiler. Involution. Springer, Berlin, 2010.

[47] O. Stormark. Lie's structural approach to PDE systems, volume 80. Cambridge University Press, 2000. 


\section{Appendix A}

\section{Tameness}

In [42] the Maurer-Cartan forms for (a certain definition of) Lie pseudo-groups were defined and their structure equations computed. The authors of that paper did not assume the same regularity assumptions as we did in our Definition 4.1.1 of a Lie pseudo-group. For them, a Lie pseudo-group is simply a pseudo-group of local diffeomorphisms on a space $\mathcal{X}$ that are local solutions to a formally integrable system of differential equations, $\mathcal{G}_{q}$, satisfying the manifold conditions from Remark 3.0.5. For the sake of clarity of the following discussion let us call such pseudo-groups smooth pseudo-groups. Since the deduction, in [42], of the structure equations for the Maurer-Cartan forms required that the linearization of $\mathcal{G}_{q}$ also be locally solvable, and since this does not follow in any trivial way from their assumptions on $\mathcal{G}_{q}$, they restricted to smooth pseudo-groups satisfying this extra condition and dubbed them tame.

To better illustrate the tameness issue, let $\mathcal{G}_{q}$ be the formally integrable determining equations for a smooth pseudo-group $\mathcal{G}$ and consider the subbundle of $T \mathcal{G}_{q}$ of vertical tangent vectors at the identity,

$$
\mathcal{W}_{q}=\left.\bigcup_{x \in \mathcal{X}} T\left(\mathcal{G}_{\left.q\right|_{x}}\right)\right|_{\left.j^{q} \mathbb{1}\right|_{x}} .
$$

Similarly, we can define $\mathcal{W}_{p}$ for any $q \leq p \leq \infty$. Recall that for a flow $\Phi_{\varepsilon} \in \mathcal{G}$ 
with $\Phi_{0}=\mathbb{1}$ its infinitesimal generator is the local vector field on $\mathcal{X}$ made up of the tangent vectors

$$
\mathbf{v}(x)=\left.\frac{d}{d \varepsilon}\right|_{\varepsilon=0} \Phi_{\varepsilon}(x) .
$$

The collection of infinitesimal generators form the Lie algebroid $\mathcal{A}$ of $\mathcal{G}$. The lift of $\mathbf{v}$ is the local (vertical) vector field, $\mathbf{V}$, on $\mathcal{G}_{\infty}$ determined by the flow

$$
j^{\infty} \varphi_{\left.\right|_{x}} \mapsto j^{\infty} \Phi_{\left.\varepsilon\right|_{\varphi(x)}} \cdot j^{\infty} \varphi_{\left.\right|_{x}},
$$

for any $j^{\infty} \varphi_{\left.\right|_{x}}$ with $\varphi(x)$ in the domain of definition of $\Phi_{\varepsilon}$. In particular, $\mathbf{V}$ at the identity is

$$
\left.\frac{d}{d \varepsilon}\right|_{\varepsilon=0}\left(j^{\infty} \Phi_{\left.\varepsilon\right|_{x}}\right) \in \mathcal{W}_{\left.\infty\right|_{x}}
$$

We say that $\mathcal{G}$ is tame if each tangent vector in $\mathcal{W}_{\infty}$ is the lift of an infinitesimal generator $\mathbf{v}$ on $\mathcal{X}$. The point of this definition is that for tame pseudo-groups there is a Lie algebra isomorphism between the Lie algebroid $\mathcal{A}$ and $\mathcal{W}_{\infty}$. Furthermore, since the Maurer-Cartan forms on the full diffeomorphism groupoid $\mathcal{D}_{\infty}(\mathcal{X})$ are dual to its Lie algebroid,

$$
\left.\bigcup_{x \in \mathcal{X}} T\left(\mathcal{D}_{\left.\infty\right|_{x}}\right)\right|_{j \infty \mathbb{1}_{x}}
$$

restricting them to $\mathcal{G}_{\infty}$ will give the correct structure equations for the Lie pseudogroup $\mathcal{G},[42]$.

As mentioned above, it is not so clear which smooth pseudo-groups are tame but one obvious case are those with locally solvable determining equations $\mathcal{G}_{q}$ such that the linearization of $\mathcal{G}_{q}$ at the identity solution is also locally solvable. We showed, in Section 3.3 , that when discussing local solvability it is very natural, indeed, necessary, to impose regularity in the sense of Definition 3.0.6. Formally integrable systems are locally solvable via the Cartan-Kähler theorem if and only if they are regular. In this appendix we show that linearization preserves the regularity of Definition 3.0.6. This implies that all pseudo-groups satisfying our Definition 4.1.1 of Lie pseudo-groups are automatically tame. Furthermore, we 
shall show that all smooth pseudo-groups are regular on an open dense and invariant subset of $\mathcal{X}$. We should mention that we do not know if there are any non-tame smooth pseudo-groups.

\section{A.1 Linearization}

We spend this subsection showing that formal integrability of a regular system $\mathcal{R}_{q}$ implies formal integrability of the linearization of $\mathcal{R}_{q}$ around any local solution. This is very simple and requires essentially only linear algebra in the form of the next lemma.

Let

$$
F=\left(F^{1}, \ldots, F^{k}\right): \mathbb{R}^{l+p} \rightarrow \mathbb{R}^{k}
$$

be smooth. Let $(w, y)$ be a typical point in $\mathbb{R}^{l+p}$, where $w \in \mathbb{R}^{l}$ and $y \in \mathbb{R}^{p}$. We write $\nabla_{y} F$ for the $k \times p$ "reduced" Jacobian matrix of $y$-derivatives of $F$. The point of the following simple lemma is to give a condition for when we can eliminate certain variables from a smooth equation.

Lemma A.1.1. Let $F$ be as above with $k<p$ and let $G: \mathbb{R}^{l+p} \rightarrow \mathbb{R}$ be smooth. Assume $\nabla_{y} F$ has full rank $k$. The vector $\nabla_{y} G(w, y)$ is locally in the span of the vectors $\nabla_{y} F^{i}(w, y)$ if and only if there exists a smooth locally defined function $K$ (of $l+k$ variables) such that $G-K(w, F)$ is a function of $w$ only. The map $K(w, F)$ vanishes on the zero set of $F$, that $i s, K(w, 0)=0$.

Proof. Let us write $y=(v, z)$, where $v \in \mathbb{R}^{p-k}$ and $z \in \mathbb{R}^{k}$. By the rank theorem, we can assume that $F$ has the form

$$
F(w, y)=F\left(w, v_{1}, \ldots, v_{p-k}, z_{1}, \ldots, z_{k}\right)=\left(z_{1}, \ldots, z_{k}\right)
$$

on an open neighborhood around 0 . For $\nabla_{y} G$ to be in the row space of $\nabla_{y} F$ then means that $G$ is a function of $w$ and $z$ only, where $z=\left(z_{1}, \ldots, z_{k}\right) \in \mathbb{R}^{k}$. But since $F=z$, we have $G=G(w, 0, F)$ so we can set $K(w, z):=G(w, 0, F)-G(w, 0,0)$ 
and we have

$$
G(w, 0, z)-K(w, F(w, v, z))=G(w, 0,0)
$$

and $K$ obviously vanishes when $F=0$.

Since the function $K$ in the above Lemma vanishes when $F=0$, there exist functions $Q^{1}, \ldots, Q^{k}$ such that $K=\sum_{i} Q^{i} F^{i}$ and Lemma A.1.1 shows that all the $y$-variables can be eliminated from $G(x, y)$ by subtracting a multiple of the $F^{i}$. That is, by a "Gaussian elimination":

$$
G(w, 0)=G(w, y)-K(w, F)=G-\sum_{i} Q^{i} F^{i}
$$

Now, let $\mathcal{R}_{q}$ be given by a collection of equations $\Phi=\left(\varphi_{1}, \ldots, \varphi_{t}\right)=0$, where $\varphi_{i}: J^{q}(\mathcal{E}) \rightarrow \mathbb{R}$. We write the Jacobian, $\nabla \Phi$, of $\Phi$ as

$$
\left[\nabla_{J} \Phi \mid \nabla_{H} \Phi\right]
$$

where $\nabla_{J} \Phi$ is made up of the columns corresponding to differentiation with respect to the jet coordinates $\left(u^{(q)}\right)$ and $\nabla_{H} \Phi$ are the columns for the (horizontal) $x$ derivatives of $\Phi$. We call $\nabla_{J} \Phi$ the jet Jacobian of $\mathcal{R}_{q}$. For $0 \leq p \leq q$, let $\nabla_{J}^{p} \Phi$ be the matrix whose columns correspond to differentiation with respect to $u_{J}^{\alpha}$, $|J|=p$. Regularity of $\mathcal{R}_{q}$ and the non-degeneracy condition from Remark 3.0 .2 imply that the jet Jacobians of all $\mathcal{R}_{q, t}^{(s)}$ are constant rank; the dimension of the null space of $\nabla_{J} \Phi$ is equal to the dimension of each fiber of $\mathcal{R}_{q} \rightarrow \mathbb{R}^{n}$, and is therefore constant by regularity. This generalizes to all $\mathcal{R}_{q, t}^{(s)}$ since these systems are always submanifolds.

Remark A.1.2. Lemma A.1.1 now implies that for regular and non-degenerate equations it is necessary and sufficient, in the search for integrability conditions, to study the jet Jacobians of $\mathcal{R}_{q}$ and the jet Jacobians of its derived equations $\mathcal{R}_{q, t}^{(s)}$. To see why, for sake of simplicity, consider the first order prolongation $\mathcal{R}_{q, 1}$. Denote the system of the purely prolonged equations $D_{i} \Phi=0,1 \leq i \leq n$, by 
$\Phi_{, 1}=0$. Choose a basis for the row space of the submatrix $\nabla_{J}^{q+1} \Phi_{, 1}$ and denote by $\mathcal{B}$ the corresponding collection of rows in $\nabla_{J} \Phi_{, 1}$. Let $\mathcal{C}$ denote the row space of $\nabla_{J} \Phi$. The presence of an integrability condition is, by Lemma A.1.1, equivalent to the jet Jacobian $\nabla_{J} \Phi_{, 1}$ containing a row that is not in the span of $\mathcal{B} \cup \mathcal{C}$.

Let $v: \mathbb{R}^{n} \rightarrow \mathbb{R}^{m}$ be a local solution to $\mathcal{R}_{q}$ and consider the linearization of $\mathcal{R}_{q}$ around $v$, i.e. the differential equation $\mathcal{L}_{v}\left(\mathcal{R}_{q}\right)$ determined by the equations

$$
\mathcal{L}_{v}\left(\varphi_{i}\right)=\left.\sum_{0 \leq|J| \leq q} \frac{\partial \varphi_{i}}{\partial u_{J}^{\alpha}}\right|_{v(x)} u_{J}^{\alpha}
$$

where $\left.\frac{\partial \varphi_{i}}{\partial u_{J}^{\alpha}}\right|_{v(x)}$ is the evaluation of $\frac{\partial \varphi_{i}}{\partial u_{J}^{\alpha}}$ on (the jets of) $v$. Notice, in particular, that $\left.\frac{\partial \varphi}{\partial u_{J}^{\alpha}}\right|_{v(x)}$ is a function of $x$ only and therefore

$$
\left.\left(D_{i} \frac{\partial \varphi}{\partial u_{J}^{\alpha}}\right)\right|_{v(x)}=\frac{\partial}{\partial x^{i}}\left(\left.\frac{\partial \varphi}{\partial u_{J}^{\alpha}}\right|_{v(x)}\right)=D_{i}\left(\left.\frac{\partial \varphi}{\partial u_{J}^{\alpha}}\right|_{v(x)}\right) .
$$

Not surprisingly, linearization commutes with prolongation.

Lemma A.1.3. $D_{i} \mathcal{L}_{v}(\varphi)=\mathcal{L}_{v}\left(D_{i} \varphi\right)$.

Proof.

$$
\begin{aligned}
D_{i} \mathcal{L}_{v}(\varphi) & =D_{i}\left(\left.\sum \frac{\partial \varphi}{\partial u_{J}^{\alpha}}\right|_{v(x)} u_{J}^{\alpha}\right) \\
& =\sum \frac{\partial}{\partial x^{i}}\left(\left.\frac{\partial \varphi}{\partial u_{J}^{\alpha}}\right|_{v(x)}\right) u_{J}^{\alpha}+\left.\sum \frac{\partial \varphi}{\partial u_{J}^{\alpha}}\right|_{v(x)} u_{J, i}^{\alpha} \\
& =\left.\sum\left(D_{i} \frac{\partial \varphi}{\partial u_{J}^{\alpha}}\right)\right|_{v(x)} u_{J}^{\alpha}+\left.\sum \frac{\partial \varphi}{\partial u_{J}^{\alpha}}\right|_{v(x)} u_{J, i}^{\alpha}
\end{aligned}
$$


And

$$
\begin{aligned}
\mathcal{L}_{v}\left(D_{i} \varphi\right) & =\mathcal{L}_{v}\left(\frac{\partial \varphi}{\partial x^{i}}+\sum \frac{\partial \varphi}{\partial u_{J}^{\alpha}} u_{J, i}^{\alpha}\right) \\
& =\left.\sum \frac{\partial^{2} \varphi}{\partial x^{i} u_{K}^{\beta}}\right|_{v(x)} u_{K}^{\beta}+\left.\sum \sum\left(\frac{\partial^{2} \varphi}{\partial u_{J}^{\alpha} u_{K}^{\beta}} u_{J, i}^{\alpha}\right)\right|_{v(x)} u_{K}^{\beta}+\left.\sum \frac{\partial \varphi}{\partial u_{J}^{\alpha}}\right|_{v(x)} u_{J, i}^{\alpha} \\
& =\left.\sum\left(D_{i} \frac{\partial \varphi}{\partial u_{K}^{\beta}}\right)\right|_{v(x)} u_{K}^{\beta}+\left.\sum \frac{\partial \varphi}{\partial u_{J}^{\alpha}}\right|_{v(x)} u_{J, i}^{\alpha},
\end{aligned}
$$

proving the lemma.

Obviously the jet Jacobian of $\mathcal{R}_{q}$ at $j^{q} v_{\left.\right|_{x}}$ and the jet Jacobian of $\mathcal{L}_{v}\left(\mathcal{R}_{q}\right)$ are the same matrix,

$$
\left(\nabla_{J} \Phi\right)_{\left.\right|_{v(x)}}=\nabla_{J}\left(\mathcal{L}_{v}(\Phi)\right)
$$

What is more, the system $\mathcal{L}_{v}\left(\mathcal{R}_{q}\right)$, being linear, will never impose any restrictions on the independent variables $x$ only, i.e. $\mathcal{L}_{v}\left(\mathcal{R}_{q}\right)$ will always satisfy the nondegeneracy condition of Remark 3.0.2. Now Lemmas A.1.1 and A.1.3 combine to prove that prolongation and projection of regular differential equations commutes with linearization.

Theorem A.1.4. Let $\mathcal{R}_{q}$ be regular and non-degenerate and let $v: \mathbb{R}^{n} \rightarrow \mathbb{R}^{m}$ be a local solution. If $\mathcal{R}_{q}$ is formally integrable, then $\mathcal{L}_{v}\left(\mathcal{R}_{q}\right)$ is formally integrable and regular.

Proof. Lemma A.1.3 implies that the $t^{\text {th }}$ order prolongation of $\mathcal{L}_{v}\left(\mathcal{R}_{q}\right),\left(\mathcal{L}_{v}\left(\mathcal{R}_{q}\right){ }_{, t}\right.$ is equal to $\mathcal{L}_{v}\left(\mathcal{R}_{q, t}\right)$. This means that the jet Jacobians of $\mathcal{L}_{v}\left(\mathcal{R}_{q}\right)_{, t}$ agree with those of $\mathcal{R}_{q, t}$ at $j^{q+t} v_{\left.\right|_{x}}$. By Remark A.1.2 these jet Jacobians contain all necessary information pertaining to integrability conditions, but then formal integrability of $\mathcal{R}_{q}$ implies that these jet Jacobians provide no integrability conditions at any order. This means that $\mathcal{L}_{v}\left(\mathcal{R}_{q}\right)$ is also formally integrable. Since $\mathcal{R}_{q, t}^{(s)}=\mathcal{R}_{q, t-s}$ for all $s \leq t$, and since $\mathcal{L}_{v}\left(\mathcal{R}_{q, t-s}\right)$ and $\mathcal{R}_{q, t-s}$ have the same symbol (and hence the same reduced Cartan characters) we can see that $\mathcal{L}_{v}\left(\mathcal{R}_{q}\right)$ is regular. 
By definition, the determining equations of a Lie pseudo-group are formally integrable and regular. The above theorem shows that their linearization are also formally integrable and regular. By the results of Section 3.3 both systems are locally solvable. This means that, in the language of [42], our definition of a Lie pseudo-group implies tameness. In the next section we show that, almost everywhere, the regularity assumption in Definition 4.1.1 can be dropped.

\section{A.2 Tameness of Lie pseudo-groups}

We now shift our focus to Lie pseudo-groups, and show that, by and large, we can drop the regularity assumption we made on their determining equations in Definition 4.1.1. More specifically, we shall prove that a smooth pseudo-group, $\mathcal{H}$, of local diffeomorphisms of $\mathcal{X}$, is regular on an open, dense, invariant set $\mathcal{X}^{\prime} \subset \mathcal{X}$ and hence are Lie thereon. This means that $\mathcal{H}$ is a Lie pseudo-group (of Definition 4.1.1) of local transformations of $\mathcal{X}^{\prime}$ and by the results from the last section it is tame.

So, let a smooth pseudo-group $\mathcal{G}$ be determined by a collection of formally integrable $q^{\text {th }}$ order equations, $\mathcal{G}_{q}$,

$$
F\left(x, X^{(q)}\right)=F\left(j^{q} \varphi_{x}\right)=0,
$$

and let $\psi \in \mathcal{G}$. Then the set $F\left(j^{q} \varphi_{\mid x}\right)=0$ is left and right invariant under the action of $\mathcal{G}$. That is, for $j^{q} \varphi_{\left.\right|_{x}} \in \mathcal{G}_{q}$ with $F\left(j^{q} \varphi_{\left.\right|_{x}}\right)=0$, we have

$$
\begin{aligned}
& F\left(R_{\psi} \cdot j^{q} \varphi_{\left.\right|_{x}}\right)=F\left(\left.j^{q}\left(\varphi \circ \psi^{-1}\right)\right|_{\psi(x)}\right)=0 \text { and } \\
& F\left(L_{\psi} \cdot j^{q} \varphi_{\left.\right|_{x}}\right)=F\left(\left.j^{q}(\psi \circ \varphi)\right|_{x}\right)=0,
\end{aligned}
$$

when the various compositions are defined. This has important consequences for the symbol of $\mathcal{G}_{q}$. Notice that when we make a change of independent variables, 
$x \mapsto y=g(x)$, the defining equations A.1 transform according to

$$
F\left(j^{q} \varphi_{\left.\right|_{x}}\right)=0 \Longleftrightarrow \bar{F}\left(j^{q}\left(\varphi \circ g^{-1}\right)_{\left.\right|_{y}}\right)=0
$$

Now, if $g=\psi$ happens to be a local transformation from $\mathcal{G}$ then A.2 tells us that the equation in the independent variables $y$ is the same as in the $x$ variables, i.e. $F=\bar{F}$. Similarly, the left action of $\psi \in \mathcal{G}$ has the same effect as changing dependent variables $X \mapsto Y=\psi(X)$. The defining equations, A.1, are therefore immune to either operation. Now let us consider the effect these operations have on the symbol of $F\left(j^{q} \varphi_{\left.\right|_{x}}\right)$ at $j^{q} \varphi_{\left.\right|_{x}} \in \mathcal{G}_{q}$, i.e. on

$$
\left.\sum_{\substack{|J|=q \\ 1 \leq i \leq n}} \frac{\partial F}{\partial X_{J}^{i}}\right|_{\left.j^{q} \varphi\right|_{x}} \xi^{J} \otimes e_{i}
$$

Changing, say, independent variables $x \mapsto y=\psi(x)$, for $\psi \in \mathcal{G}$, and denoting the Jacobian of $\psi$ by $\nabla \psi, \xi=\left(\xi^{1}, \ldots, \xi^{n}\right)$, viewed as a column vector, transforms according to

$$
\xi \mapsto \nabla \psi^{-T} \cdot \bar{\xi}
$$

where $-T$ denotes the matrix inverse transpose and $\bar{\xi}=\left(\bar{\xi}^{1}, \ldots, \bar{\xi}^{n}\right)$. The symbol A.3 then transforms according to

$$
\left.\sum_{\substack{|J|=q \\ 1 \leq i \leq n}} \frac{\partial F}{\partial X_{J}^{i}}\right|_{\left.j^{q} \varphi\right|_{x}}\left(\nabla \psi^{-T} \cdot \bar{\xi}\right)^{J} \otimes e_{i}
$$

On the other hand, changing independent variables $x \mapsto y=\psi(x)$ "transforms" the differential equation $F\left(j^{q} \varphi_{\left.\right|_{x}}\right)=0$ to $F\left(\left.j^{q}(\varphi \circ \psi)\right|_{y}\right)=0$, and hence the symbol according to

$$
\left.\sum_{\substack{|J|=q \\ 1 \leq i \leq n}} \frac{\partial F}{\partial \bar{X}_{J}^{i}}\right|_{\left.j^{q}\left(\varphi \circ \psi^{-1}\right)\right|_{y}} \bar{\xi}^{J} \otimes e_{i},
$$


where

$$
\bar{X}_{J}^{i}=\frac{\partial^{|J|}}{\partial y^{J}}\left(\varphi \circ \psi^{-1}\right) .
$$

Notice that $($ A.5 $)$ is simply the symbol of the determining equation at $\left.j^{q}\left(\varphi \circ \psi^{-1}\right)\right|_{y}$. Comparing (A.4) and A.5 we can see that the symbol of $F\left(j^{q} \varphi_{\mid x}\right)=0$ at $j^{q}\left(\varphi \circ \psi^{-1}\right)_{\mid y}$ is obtained from the symbol at $j^{q} \varphi_{\mid x}$ by the change of variables

$$
\xi \mapsto \nabla \psi^{-T} \cdot \bar{\xi}
$$

Since the reduced Cartan characters for the symbol of $\mathcal{G}_{q}$ are coordinate independent quantities we have established that they are the same at $j^{q} \varphi_{x}$ and $j^{q}\left(\varphi \circ \psi^{-1}\right)_{\mid \psi(x)}$. The same argument as above will show that the reduced Cartan characters of the symbol of $\mathcal{G}_{q}$ at $j^{q} \varphi_{\left.\right|_{x}}$ are the same as those at $j^{q}(\psi \circ \varphi)_{\mid x}$ for $j^{q} \psi_{\varphi(x)} \in \mathcal{G}_{q}$. These considerations will provide an easy proof of our main theorem, but first we need a definition.

Definition A.2.1. Let $\mathcal{G}$ be a pseudo-group of local diffeomorphisms of $\mathcal{X}$. We say that two points $x, y \in \mathcal{X}$ are in the same $\mathcal{G}$-orbit if they are in the same minimal invariant set. This means that there there is a finite sequence of $\psi_{i} \in \mathcal{G}$ such that $y=\psi_{1} \circ \ldots \circ \psi_{t}(x)$. If all pairs of points in $\mathcal{X}$ are in the same $\mathcal{G}$-orbit, we say that $\mathcal{G}$ is transitive.

Theorem A.2.2. Let $\mathcal{G}$ be a smooth pseudo-group determined by a formally integrable system $\mathcal{G}_{q}$. Then $\mathcal{G}_{q}$ is regular on an invariant open dense set (and hence tame thereon).

Proof. We already know from Section 3.3 that these equations are regular on a dense open set $U \subset \mathcal{G}_{q}$, so we only need to show them to be invariant. Consider $j^{q} \psi_{\left.\right|_{x}} \in U$. Then, by the above discussion, the symbol of $\mathcal{G}_{q}$ at $j^{q} \psi_{\left.\right|_{x}}$ has the same reduced Cartan characters as the symbol at $j^{q} \mathbb{1}_{\mid x}$. Now, for any $j^{q} \varphi_{\mid x} \in \mathcal{G}_{q}$, with $\varphi(x)=y$, we have that

$$
j^{q} \mathbb{1}_{\left.\right|_{y}}=\left.j^{q} \varphi_{x} \cdot j^{q} \mathbb{1}_{\left.\right|_{x}} \cdot j^{q} \varphi^{-1}\right|_{y},
$$


as so the reduced Cartan characters of the symbol of $\mathcal{G}_{q}$ at $j^{q} \mathbb{1}_{\left.\right|_{x}}$ are the same as those at $j^{q} \mathbb{1}_{\left.\right|_{y}}$. This holds for all pairs of points $x, y$ for which there is a $\varphi \in \mathcal{G}$ such that $\varphi(x)=y$. Since the symbol has constant reduced Cartan characters on each horizontal fiber $\mathcal{G}_{\left.q\right|_{z}}$ this proves the theorem.

Corollary A.2.3. Formally integrable determining equations, $\mathcal{G}_{q}$, for transitive smooth pseudo-groups are regular (and hence tame).

Example A.2.4. Consider the Lie pseudo-group of local diffeomorphisms of $\mathbb{R}^{2}$ whose elements solve the system of equations

$$
X_{y}=0, \quad Y_{y}=X_{x}
$$

The general solution to this system has the form

$$
X=f(x), \quad Y=f^{\prime}(x) y+g(x),
$$

where $f$ is a local diffeomorphism of $\mathbb{R}$ and $g$ is arbitrary (recall, though, that we require all our maps to be real analytic). Now change independent variables $(x, y) \mapsto(\bar{x}, \bar{y})=\psi(x, y)$ where $\psi \in \mathcal{G}$. Let $A$ denote the Jacobian of $\psi$,

$$
A=\left[\begin{array}{cc}
\bar{x}_{x} & \bar{x}_{y} \\
\bar{y}_{x} & \bar{y}_{y}
\end{array}\right]=\left[\begin{array}{cc}
\bar{x}_{x} & 0 \\
\bar{y}_{x} & \bar{x}_{x}
\end{array}\right] .
$$

The second equality follows from the fact that $\psi \in \mathcal{G}$ must solve the determining equations. For a function $g: \mathbb{R}^{2} \rightarrow \mathbb{R}$ we have the change of variables formula

$$
\left[\begin{array}{l}
g_{x} \\
g_{y}
\end{array}\right]=A^{-T} \cdot\left[\begin{array}{l}
g_{\bar{x}} \\
g_{\bar{y}}
\end{array}\right],
$$

where

$$
A^{-T}=\left[\begin{array}{ll}
x_{\bar{x}} & y_{\bar{x}} \\
x_{\bar{y}} & y_{\bar{y}}
\end{array}\right]=\left[\begin{array}{cc}
x_{\bar{x}} & y_{\bar{x}} \\
0 & x_{\bar{x}}
\end{array}\right] .
$$


So in the new independent variables the determining equations for elements of $\mathcal{G}$ become

$$
X_{y}=0 \Leftrightarrow X_{\bar{y}} \cdot x_{\bar{x}}=0 \Leftrightarrow X_{\bar{y}}=0,
$$

and (using $X_{\bar{y}}=0$ )

$$
Y_{y}-X_{x}=0 \quad \Leftrightarrow \quad Y_{\bar{y}} x_{\bar{x}}-X_{\bar{x}} x_{\bar{x}}-X_{\bar{y}} y_{\bar{x}}=0 \quad \Leftrightarrow \quad Y_{\bar{y}}-X_{\bar{x}}=0 .
$$

So, as we expected, the determining equations have the exact same form after changing independent variables according to $\psi \in \mathcal{G}$.

Notice that the symbol of the equations $\mathcal{G}_{1}$, A.6), in the polynomial module $\mathbb{R}\left[\xi^{1}, \xi^{2}\right] \otimes \mathbb{R}^{2}$ is constant on $\mathcal{G}_{q}$,

$$
\xi^{2} \otimes e_{1}, \quad \xi^{2} \otimes e_{2}-\xi^{1} \otimes e_{1}
$$

where $\left\{e_{1}, e_{2}\right\}$ is the standard basis for $\mathbb{R}^{2}$. By the discussion preceding Theorem A.2.2 we therefore expect this symbol to be genuinely invariant under change of variables coming from $\mathcal{G}$. Changing independent variables $(x, y) \mapsto(\bar{x}, \bar{y})=$ $\psi(x, y)$ will induce a transformation on the $\xi^{1}$ and $\xi^{2}$ according to

$$
\left[\begin{array}{l}
\xi^{1} \\
\xi^{2}
\end{array}\right]=A^{-T} \cdot\left[\begin{array}{l}
\bar{\xi}^{1} \\
\bar{\xi}^{2}
\end{array}\right]=\left[\begin{array}{c}
x_{\bar{x}} \bar{\xi}^{1}+y_{\bar{x}} \bar{\xi}^{2} \\
x_{\bar{x}} \bar{\xi}^{2}
\end{array}\right] .
$$

The symbol A.7 then transforms to

$$
\left(x_{\bar{x}} \bar{\xi}^{2}\right) \otimes e_{1}, \quad\left(x_{\bar{x}} \bar{\xi}^{2}\right) \otimes e_{2}-\left(x_{\bar{x}} \bar{\xi}^{1}+y_{\bar{x}} \bar{\xi}^{2}\right) \otimes e_{1},
$$

which, after some autoreduction becomes

$$
x_{\bar{x}}\left(\bar{\xi}^{2} \otimes e_{1}\right), \quad x_{\bar{x}}\left(\bar{\xi}^{2} \otimes e_{2}-\bar{\xi}^{1} \otimes e_{1}\right) .
$$

Comparing (A.8) with (A.7), we can see that, up to autoreduction and scalar 
multiples, the symbol is the same in the new variables $\bar{\xi}$.

Remark A.2.5. Our definition of a Lie pseudo-group, as opposed to that of a smooth pseudo-group, has the advantage of automatically being tame. One example of the benefits of our definition in conjunction with the results above is a trivial proof of the fact that any effective action of a finite dimensional Lie group, $G$, on a manifold is regular (and hence tame). This is simply because the determining equations for the elements of $G$ must be maximally over-determined and hence the reduced Cartan characters will be constant and zero above a certain order. See the next example for more.

The following example was used by Cartan, [7, p. 1357], to illustrate his theory of essential invariants, i.e. non constant coefficients appearing in the structure equations for a Lie pseudo-group. The Lie pseudo-group $\mathcal{G}$ in this example is very simple as it comes from the action of a two dimensional abelian Lie group, $G$, on $\mathbb{R}^{3}$ and this simplicity ( $G$ has trivial structure equations) should be reflected in the pseudo-group structure equations. However these include non constant coefficients. This "discrepancy" is explained by the fact that the structure equations of $\mathcal{G}$ and $G$ are only really isomorphic on each $\tau$-vertical fiber since $G$ acts freely and transitively on them (and not on the entire set $\mathcal{G}_{\infty}$ ).

Example A.2.6. Consider the following Lie pseudo-group coming from the action of the abelian Lie group $\mathbb{R}^{2}$ on $\mathbb{R}^{3}$ given by

$$
X=x, \quad Y=y, \quad U=u+a x+b x,
$$

where $(a, b) \in \mathbb{R}^{2}$. The determining equations, $\mathcal{G}_{1}$ for these transformations is

$$
\begin{gathered}
X=x, \quad Y=y, \quad U=u+x U_{x}+y U_{y}, \quad X_{x}=1, \quad Y_{y}=1, \quad U_{u}=1, \\
X_{y}=X_{u}=Y_{x}=Y_{u}=0
\end{gathered}
$$


but at the second order we have

$$
X_{i j}=Y_{i j}=U_{i j}=0, \quad \text { for all } i, j \in\{x, y, u\} .
$$

These determining equations are regular with $p^{*}=2$. All second order MaurerCartan forms are zero and the first order forms satisfy

$$
\mu^{x}=\mu^{y}=\mu_{x}^{x}=\mu_{y}^{x}=\mu_{u}^{x}=\mu_{x}^{y}=\mu_{y}^{y}=\mu_{u}^{y}=\mu_{u}^{u}=0
$$

and

$$
\mu^{u}=X \mu_{x}^{u}+Y \mu_{y}^{u}
$$

If we were to restrict our attention to the local action of $\mathcal{G}$ on, say, the subset of $\mathbb{R}^{3}$ where $y \neq 0$, we could write

$$
\mu_{y}^{u}=\frac{1}{Y} \mu^{u}-\frac{X}{Y} \mu_{x}^{u}
$$

and we can see that the structure equations on $\mathcal{G}_{1}$ will involve the coefficients $\frac{1}{Y}$ and $\frac{X}{Y}$. However, as is easily checked, once we restrict to any $\tau$-vertical fiber we obtain (taking $\mu_{x}^{u}$ and $\mu_{y}^{u}$ as a basis on each such fiber) the correct structure equations

$$
d \mu_{x}^{u}=d \mu_{y}^{u}=0
$$

